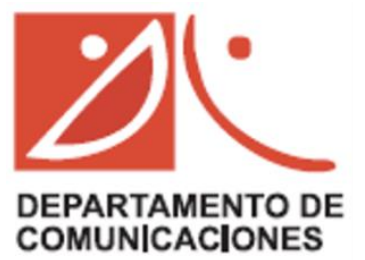

Universitat Politècnica València

Departamento de Comunicaciones

\title{
Dispositivos emisores de luz para transmisión óptica de alta velocidad
}

\section{Roberto Alejandro Larrea Luzuriaga}

\author{
Tutor: Dr. Pablo Sanchis Kilders \\ Dr. Ana María Gutiérrez Campo
}

Tesis presentada para la obtención del grado de Doctor en Telecomunicaciones de la Universitat Politècnica de València.

Thesis submitted to the Universitat Politècnica de València in partial fulfillment of the requirements for the degree of Doctor of Philosophy in Telecommunications Engineering. 

A quien me inspira a superarme cada día más.

Mi hijo Roberto Joaquín 



\section{Agradecimientos}

Mi más sincero agradecimiento a mi tutor y director de tesis Pablo, por la confianza puesta en mi persona para el desarrollo de las tareas encomendadas en el presente trabajo de investigación a lo largo de estos 4 años. Por su profesionalismo y mística como educador e investigador que ha representado para mí un referente. Además, de su gran calidad humana, su valiosa ayuda y ánimo en los momentos difíciles, acciones que siempre las llevaré presentes. Un especial agradecimiento a mi cotutora Ana, quien ha estado presente en todo el desarrollo de la investigación. Por el arduo trabajo puesto para la consecución de los objetivos trazados, por todos los conocimientos compartidos y por toda la ayuda brindada para mi desarrollo como investigador, mi gran estima.

También quiero dar un agradecimiento a todo el equipo de fabricación en sala limpia Rafa, Amadeu, Juan, Laurent y al equipo de encapsulado y empaquetamiento Jose, Aina, Pau. Por todo su profesionalismo, trabajo y valioso esfuerzo realizado para la fabricación y preparación de las muestras en cada una de las etapas de la investigación, y obtener los mejores resultados. Sin duda su labor ha sido esencial para alcanzar los objetivos de esta tesis. De la misma manera, un agradecimiento a David por toda la ayuda y soporte brindado en los laboratorios de caracterización, y a todo el personal del Centro de Tecnología Nanofotónica NTC, por su valiosa labor en pro de la investigación.

Fuera del centro de trabajo, un agradecimiento especial a mi hijo Joaquín y a su madre Valeria por todo su apoyo y paciencia durante todo este tiempo que no he estado presente, ustedes han sido la razón de mi esfuerzo y superación de cada día. Quiero agradecer también a toda mi familia, a mis padres y hermanos por su apoyo incondicional y ánimo, que me han permitido seguir siempre adelante en todas las etapas de mi vida. 



\section{Resumen}

La fotónica de silicio es una de las tecnologías con más auge en las últimas dos décadas. Es por ello que el estudio y desarrollo de circuitos integrados fotónicos ha tenido una importante presencia en la investigación de nuevos componentes y dispositivos para la mejora de prestaciones en sistemas de comunicaciones ópticos. Una de las principales ventajas de la fotónica de silicio es su compatibilidad con la tecnología electrónica CMOS, la cual la convierte en una plataforma prometedora para la fabricación en masa a bajo coste. El tamaño reducido de las estructuras fotónicas y las bajas pérdidas alcanzadas en guías de onda nanométricas ha permitido el desarrollo de dispositivos con una alta densidad de integración. De igual manera, se han demostrado moduladores ópticos de alta velocidad y fotodetectores altamente eficientes. Sin embargo, el desarrollo de la fuente de luz o láser, elemento indispensable en cualquier sistema de comunicaciones óptico, no es posible debido al "bandgap" indirecto del silicio. Por lo cual, otros materiales de "bandgap" directo se han estudiado y propuesto para conseguir integrar un láser sobre el chip de silicio con emisión en las bandas $\mathrm{O}$ y $\mathrm{C}$. El principal desafío radica en alcanzar el régimen de emisión estimulada coherente por medio de un bombeo eléctrico y de forma eficiente. Para ello se han propuesto varios esquemas de integración. La integración monolítica aborda la fabricación del láser directamente en el chip. Por otra parte, la integración híbrida consiste en la fabricación del láser por separado para luego ser integrado en el chip, siendo este esquema con el que mejores resultados se han conseguido. De esta manera, el principal objetivo de la tesis ha sido el desarrollo de un láser de cavidad externa por medio de un esquema de integración híbrida y con funcionamiento en la banda $\mathrm{O}$. Los distintos bloques básicos se han analizado, diseñado, fabricado y demostrado experimentalmente. Finalmente, se ha llevado a cabo la integración con el medio de ganancia demostrándose satisfactoriamente emisión estimulada y funcionamiento monomodo. Además, en la tesis se ha abordado también el acoplamiento a estructuras de guiado tipo ranura, las cuales han sido propuestas en esquemas de integración monolítica. 



\section{Resum}

La fotònica de silici és una de les tecnologies amb més auge en les últimes dues dècades. És per això que l'estudi i desenvolupament de circuits integrats fotònics ha tingut una important presència a la investigació de nous components i dispositius per a la millora de prestacions en sistemes de comunicacions òptics. Una de les principals avantatges de la fotònica de silici és la seva compatibilitat amb la tecnologia electrònica CMOS, la qual la converteix en una plataforma prometedora per a la fabricació en massa a baix cost. La mida reduïda de les estructures fotòniques i les baixes pèrdues assolides en guies d'ona nanomètriques ha permès el desenvolupament de dispositius amb una alta densitat d'integració. De la mateixa manera, s'han demostrat moduladors òptics d'alta velocitat i fotodetectors altament eficients. No obstant això, el desenvolupament de la font de llum o làser, element indispensable en qualsevol sistema de comunicacions òptic, no és possible a causa del "bandgap" indirecte del silici. Per la qual cosa, altres materials de "bandgap" directe s'han estudiat i proposat per aconseguir integrar un làser sobre el xip de silici amb emissió en les bandes O i C. El principal desafiament rau a aconseguir el règim d'emissió estimulada coherent per mitjà de un bombament elèctric i de forma eficient. Per a això s'han proposat diversos esquemes d'integració. La integració monolítica aborda la fabricació del làser directament en el xip. D'altra banda, la integració híbrida consisteix en la fabricació del làser per separat per després ser integrat en el xip, sent aquest esquema amb el que millors resultats s'han aconseguit. D'aquesta manera, el principal objectiu de la tesi ha sigut el desenvolupament d'un làser de cavitat externa per mitjà d'un esquema d'integració híbrida i amb funcionament a la banda $\mathrm{O}$. Els diferents blocs bàsics s'han analitzat, dissenyat, fabricat i demostrat experimentalment. Finalment, s'ha dut a terme la integració amb el medi de guany demostrant satisfactòriament emissió estimulada i funcionament monomode. A més, en la tesi s'ha abordat també l'acoblament a estructures de guiatge tipus ranura, les quals han estat proposades en esquemes d'integració monolítica. 



\section{Abstract}

Silicon photonics is one of the technologies that has raised a higher interest during the last two decades. Therefore, the development of photonic integrated circuits has had an important presence in the research of new components and devices for the improvement of performance in optical communication systems. One of the main advantages of silicon photonics is its compatibility with CMOS electronic technology, which makes it a promising platform for mass production at low cost. The reduced size of photonic structures and the low propagation losses achieved in nanometric photonic waveguides has allowed the development of devices with a high density of integration. Similarly, high-speed optical modulators and highly efficient photodetectors have been demonstrated. However, the development of the light source or laser, an indispensable element in any optical communication system, is not possible due to the indirect bandgap of silicon. Therefore, other direct bandgap materials have been studied and proposed to integrate a laser on the silicon chip with emission in the $\mathrm{O}$ and $\mathrm{C}$ bands. The main challenge lies in reaching the coherent stimulated emission regime by means of an electric pump and in an efficient way. For this, several integration schemes have been proposed. Monolithic integration deals with the fabrication of the laser directly on the chip. On the other hand, hybrid integration consists in the fabrication of the laser separately to later be integrated into the chip. The best results have been achieved by means of this approach. Therefore, the main goal of the thesis has been the development of an external cavity laser by means of a hybrid integration scheme and with operation in the O-band. The different basic building blocks have been analyzed, designed, fabricated and experimentally demonstrated. Finally, the integration with the active material has been carried out, successfully demonstrating stimulated emission and single-mode operation. In addition, the thesis has also addressed the coupling to slow waveguide guidance structures, which have been proposed in monolithic integration schemes. 



\section{Índice}

\section{Agradecimientos}

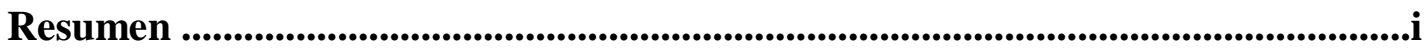

Resum

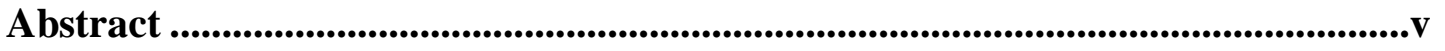

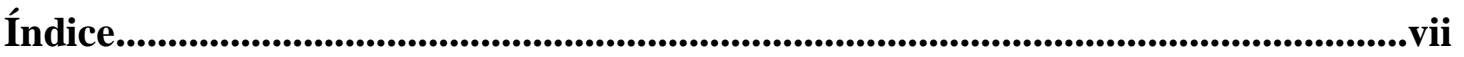

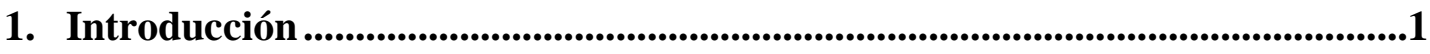

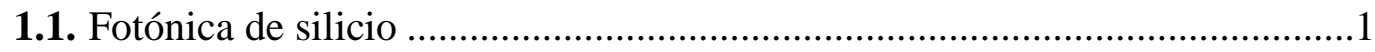

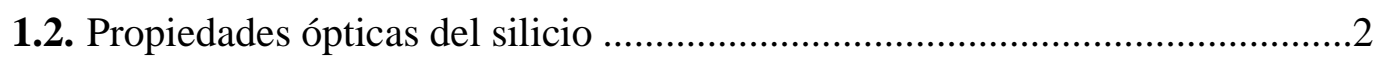

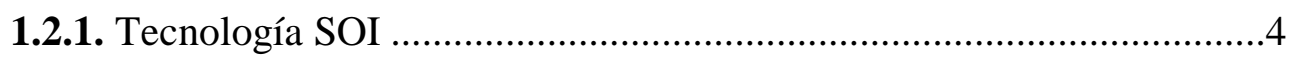

1.2.2. Guías de onda sobre silicio............................................................5

1.2.3. Estructuras para el acoplamiento de luz en una guía............................5

1.2.3.1. Acoplador mediante convertidor de tamaño de modo ........6

1.2.3.2. Acoplador mediante red de difracción ................................ 7

1.3. Láseres integrados sobre fotónica de silicio ...............................................9

1.3.1. Integración monolítica sobre silicio ............................................... 10

1.3.2. Integración híbrida sobre silicio.........................................................10

1.3.3. Estado del arte de láseres con cavidad externa ...................................12

1.4. Objetivos y organización de la tesis ........................................................14

2. Acoplador de luz a fibra para esquemas de integración monolítica.................17

2.1. Descripción de guía tipo ranura considerada .................................................17

2.2. Diseño del acoplador a fibra basado en red de difracción ..............................19

2.3. Fabricación y resultados experimentales......................................................23

3. Diseño esquema de integración híbrida de láser con cavidad externa .............27

3.1. Descripción del esquema propuesto ...........................................................27

3.2. Diseño del acoplador a láser basado en convertidor de tamaño de modo.......28

3.2.1. Especificaciones del QD.........................................................29

3.2.2. Análisis de la condición de emisión estimulada..............................37

3.3. Diseño del filtro óptico basado en anillo resonante ......................................44

3.4. Diseño del reflector basado en red de difracción .............................................50

3.5. Diseño del mecanismo de sintonización termo-óptica ....................................58

3.6. Diseño del acoplador a fibra basado en red de difracción ..............................61

3.7. Diseño del acoplador a fibra basado en convertidor de tamaño de modo.......64 
4. Fabricación y resultados experimentales del esquema propuesto de integración híbrida de láser con cavidad externa ...........................................................68

4.1. Descripción del montaje de medidas .............................................................69

4.1.1. Montaje para el acoplamiento horizontal ........................................70

4.1.2. Montaje para el acoplamiento vertical.........................................71

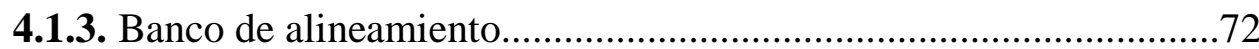

4.2. Fabricación y resultados experimentales de bloques básicos..........................72

4.2.1. Caracterización del acoplador mediante red de difracción...............73

4.2.2. Caracterización del acoplador de tamaño de modos a fibra .............74

4.2.3. Caracterización del filtro óptico basado en anillo ...........................76

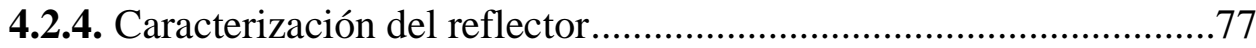

4.3. Desarrollo y caracterización del láser con cavidad externa..............................79

4.3.1. Caracterización del QD y acoplador de tamaño de modos..............81

4.3.2. Caracterización de la cavidad externa ...........................................83

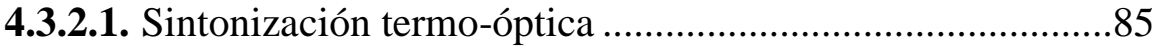

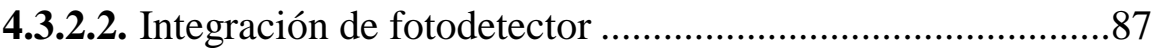

4.3.3. Demostración del funcionamiento del láser ....................................89

5. Conclusiones y perspectivas futuras .....................................................................95

Lista de publicaciones ...........................................................................................98

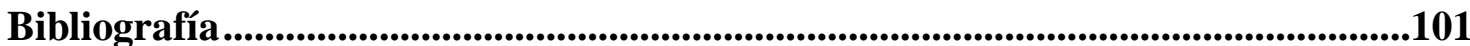





\section{Capítulo 1}

\section{Introducción}

\subsection{Fotónica de silicio}

La invención de la fuente de luz coherente en 1960, por medio de la amplificación de luz de emisión estimulada o LASER (Light Amplification by Stimulated Emission of Radiation) fue un gran hito que marcó el desarrollo de múltiples aplicaciones en diversas áreas. Entre ellas destaca, sin lugar a dudas, el área de las aplicaciones fotónicas u optoelectrónicas. En el sector de las comunicaciones, el láser revolucionó la manera de transmitir información, de forma que la industria de la fibra óptica, como medio para propagar luz, emergió como la solución ideal para establecer enlaces de larga y corta distancia con mejores prestaciones, como bajo retardo, mayor ancho de banda e inmunidad a interferencias electromagnéticas, y que dio lugar a la proliferación de redes de comunicaciones ópticas de área local y global [1]. Sin embargo, la demanda creciente de información y la aparición de nuevas tecnologías con mayores prestaciones y mayor consumo de ancho de banda está provocando cuellos de botella en los nodos de conmutación. Por tal razón, la tecnología electrónica y fotónica se han ido desarrollando para solventar estos problemas. Por una parte, la microelectrónica mediante la integración de componentes o circuitos integrados se ha esforzado cada vez más en incorporar un mayor número de componentes por unidad de área para mejorar y crear nuevos dispositivos con mayor capacidad de procesamiento y memoria en un reducido tamaño. Sin embargo, esta reducción de tamaño y el número creciente de transistores por circuito integrado incrementan el sobrecalentamiento que puede deteriorar el funcionamiento y reducir el tiempo de vida. Además, la integración de transistores por unidad de área tiene un límite, por lo cual otras tecnologías se han desarrollado como alternativa a la creciente demanda de información [2]. En dicho contexto, la tecnología fotónica es una alternativa que permite mejorar las capacidades de los sistemas de comunicaciones, de manera que interconexiones ópticas entre dispositivos, tarjetas de comunicación o incluso entre circuitos integrados chip a chip han sido estudiadas y desarrolladas. La implementación 


\section{Capítulo 1: Introducción}

de circuitos integrados fotónicos o PICs (Photonic Integrated Circuits) se ha llevado a cabo reduciendo su tamaño a escalas nanométricas, lo que contribuye a reducir el consumo de energía, sobrecalentamiento y espacio. Sin embargo, la integración de la tecnología microelectrónica y fotónica no es sencilla y puede incrementar el coste resultando en soluciones que no son económicamente viables. De esta manera, la fotónica de silicio se presenta como la plataforma ideal para dicha integración por su compatibilidad con los procesos de fabricación de la tecnología electrónica CMOS (Complementary Metal Oxide Semiconductor) al utilizar el mismo substrato para su desarrollo [3]. En estos últimos años, se han demostrado grandes avances en el desarrollo de componentes basados en fotónica de silicio con grandes perspectivas de funcionamiento, como guías de onda de bajas pérdidas [4], moduladores de alta velocidad [5], y detectores con un gran ancho de banda [6]. Así mismo, se ha demostrado su potencial para implementar sistemas de alta densidad de interconexiones ópticas llegando a transmisiones por unidad de área sobre los $27 \mathrm{Tbs} \cdot \mathrm{cm}^{-2}$ [7].

\subsection{Propiedades ópticas del silicio}

El silicio ( $\mathrm{Si}$ ) es uno de los materiales más abundantes en la corteza terrestre. Se clasifica dentro del grupo IV de los semiconductores metaloides. Uno de los aspectos más importantes en óptica es mantener el mayor control posible sobre la interacción de la luz y el material sobre el cual se propaga. Por ello, las propiedades ópticas del material tienen un alto impacto sobre el comportamiento de la luz. En este contexto, el índice de refracción, $n$, es la principal propiedad de un material óptico cuyo valor varía en función de la longitud de onda de propagación, $\lambda$. Su valor se corresponde con un número complejo, cuya parte real determina la velocidad de fase de la luz sobre el material dieléctrico. Por otro lado, la parte imaginaria refleja la ganancia del medio o pérdidas determinadas por el coeficiente de extinción o índice de absorción. El silicio se caracteriza por un alto índice de refracción y un bajo nivel de absorción en el espectro del infrarrojo, tal como se observa en la figura 1.1(a) [8].

Sin embargo, el índice de refracción de un material óptico no es un parámetro constante, y su valor puede variar dependiendo de la temperatura. La variación del índice de refracción en función de la variación de temperatura determina el coeficiente termoóptico del material $d n / d T$, siendo $n$ el índice de refracción y $T$ la temperatura. Este valor depende también ligeramente de la longitud de onda tal y como se puede observar en la figura 1.1(b) [9]. 

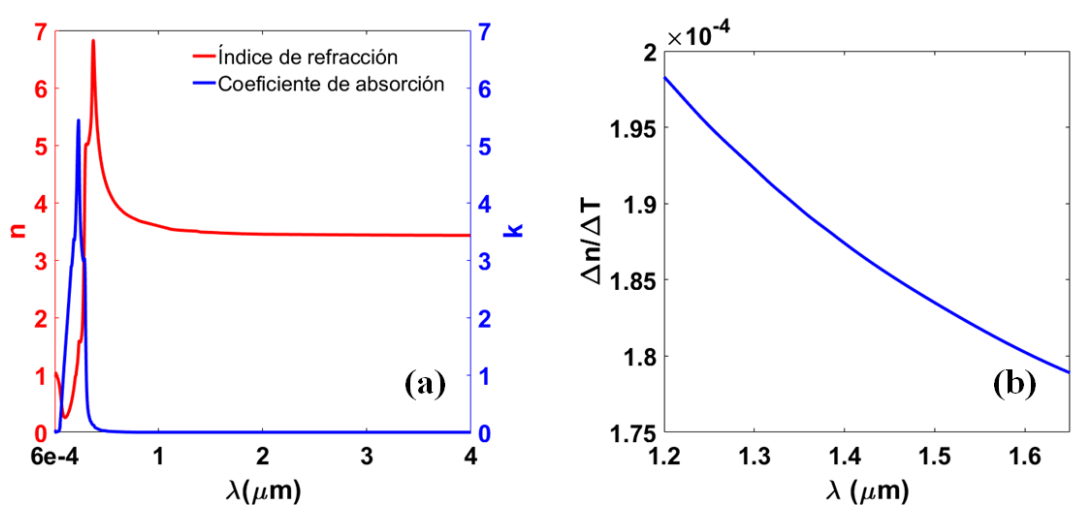

Figura 1.1: (a) Índice de refracción y coeficiente de absorción, (b) coeficiente termo-óptico del silicio.

Otra de las propiedades electroópticas de los materiales semiconductores es la de servir de fuentes emisoras de luz, proporcionando ganancia y/o emisión espontanea al aplicar un potencial eléctrico a un material semiconductor formado por una unión p-n. De este modo, al polarizar la unión de manera directa, se inyectan electrones de la zona de tipo $\mathrm{n}$ hacia la zona de tipo $\mathrm{p}$, y huecos de la zona $\mathrm{p}$ a la zona $\mathrm{n}$, generando una alta recombinación de electrones y huecos, dando lugar a la emisión espontánea y/o estimulada, tal como se puede observar en la figura 1.2. El primer caso, es el principio de funcionamiento de los diodos emisores de luz o LED (Light Emitting Diode), en el cual un electrón de la banda de conducción (BC) pasa a la banda de valencia (BV) produciendo una recombinación electrón-hueco. Por consiguiente, un fotón se emite de manera espontánea. El segundo caso corresponde al principio de los láseres y amplificadores ópticos, de tal manera que un fotón que atraviesa el material estimula una recombinación electrón-hueco, generándose un nuevo fotón [10].
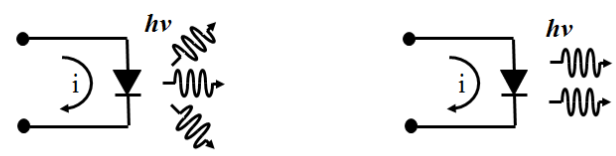

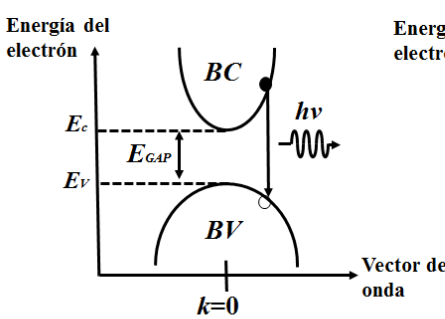

(a)

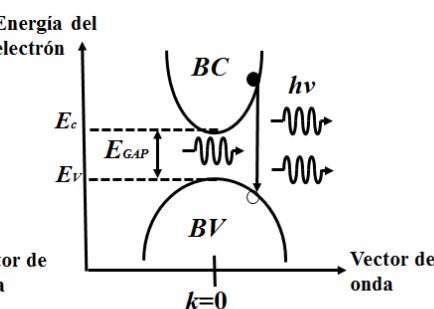

(b)

Figura 1.2: (a) Emisión espontánea, (b) Emisión estimulada.

Los semiconductores pueden clasificarse de acuerdo con su eficiencia como emisores de luz en "gap" directo y "gap" indirecto, tal como se muestra en la figura 1.3. El silicio es un semiconductor de "gap" indirecto, resultando no eficiente para la implementación de emisores ópticos, debido a que no pueden realizarse transiciones directas de una banda a otra, requiriéndose efectos adicionales para compensar la diferencia de vector de onda 


\section{Capítulo 1: Introducción}

entre bandas. Los semiconductores de "gap" directo, correspondiente a materiales de los grupos III-V y II-VI, son altamente eficientes y resultan idóneos para la realización de emisores de luz.

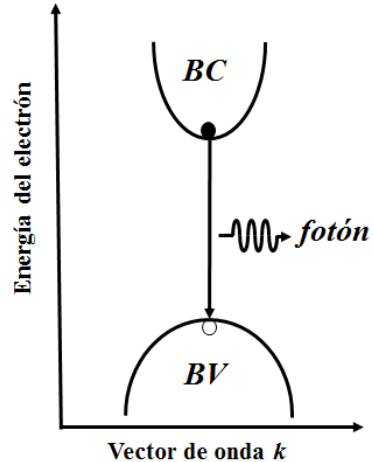

(a)

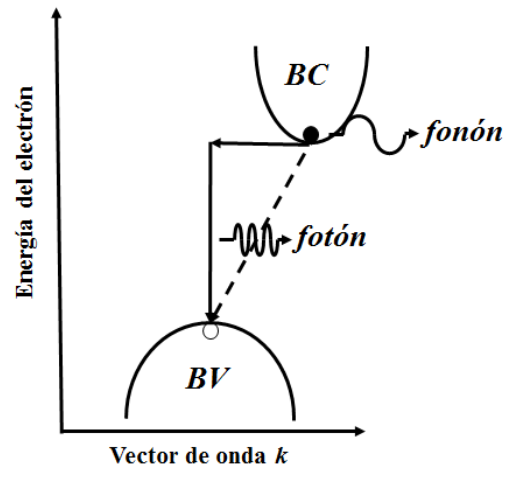

(b)

Figura 1.3: Semiconductores (a) Gap directo, (b) Gap indirecto.

\subsubsection{Tecnología SOI}

La tecnología SOI (Silicon On Insulator) es una tecnología basada en silicio que destaca por su alto contraste de índices de refracción entre los dos materiales que lo componen. El silicio, que suele utilizarse como núcleo, tiene un índice de refracción de 3.5 y el óxido, que suele utilizarse como cubierta, tiene un índice de refracción de 1.44, para una longitud de onda de 1310nm. El alto contraste de índices permite un mayor confinamiento de la luz y por tanto la fabricación de guías de onda de dimensiones reducidas. Sin embargo, las pérdidas de propagación se incrementan principalmente por la rugosidad de las paredes de la guía que dependen en gran parte del proceso de fabricación. Las dimensiones alcanzadas mediante esta tecnología, del orden de nanómetros, son de gran interés para la implementación de circuitos integrados fotónicos, lo que permite reducir aún más el tamaño de los dispositivos de comunicaciones, incrementar sus prestaciones y hacerlos energéticamente eficientes, al utilizar la luz y todas las ventajas que conlleva la tecnología óptica [11].

Una de las características precursoras del desarrollo de la tecnología SOI es su compatibilidad con la tecnología electrónica CMOS. Debido a que ambas tecnologías utilizan el mismo substrato, es posible aprovechar el desarrollo en los procesos de fabricación de la tecnología microelectrónica. Además, el bajo coste que esto supone debido a la materia prima utilizada y su producción en masa [12]. Existen obleas SOI de manera comercial de diverso espesor y tamaño. Una oblea consiste en una delgada capa de silicio cuyo espesor puede ser de 200-250nm, depositada sobre una capa de óxido de silicio $\mathrm{SiO}_{2}$ de espesor de $1-3 \mu \mathrm{m}$, la cual a su vez se encuentra depositada sobre un substrato de silicio de un espesor de alrededor de $750 \mu \mathrm{m}$. 


\subsubsection{Guías de onda sobre silicio}

Una guía es un medio guiado capaz de transportar una onda a una distancia dada. En comunicaciones ópticas esto es posible a través del confinamiento de la luz sobre una estructura multicapa con diferentes índices de refracción, tal como se muestra en la figura 1.4. Por medio del efecto de reflexión total interna o TIR (Total Internal Reflection), la luz es confinada y guiada en la capa de mayor índice de refracción (núcleo) rodeada por otra capa de menor índice de refracción (cubierta). Una de las propiedades de las guías de onda es la de mantener la polarización del modo de propagación acoplado en la guía en todo el trayecto en el cual se propaga. Es así como se puede propagar la luz sobre la guía tanto en polarización eléctrica TE (Transversal Electric) como en polarización magnética TM (Transversal Magnetic). Además, las guías pueden ser monomodo o multimodo, dependiendo de la geometría de la estructura, siendo las más utilizadas en comunicaciones ópticas las guías monomodo que solo permiten la propagación de un modo fundamental con polarización TE y/o TM [13].

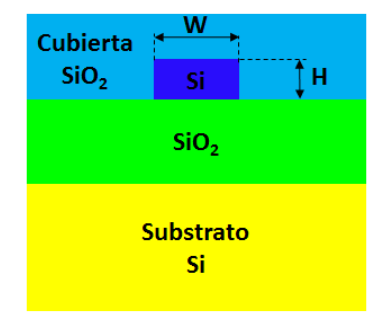

(a)

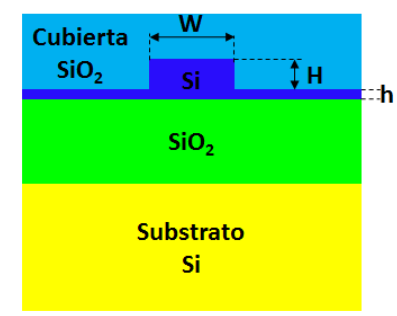

(b)

Figura 1.4: Guía enterrada SOI (a) "Strip", (b) "Rib”.

El tipo de guía utilizada en esta tesis corresponde a una guía SOI tipo "strip" monomodo con espesor $\mathrm{H}=220 \mathrm{~nm}$ y anchura $\mathrm{W}=500 \mathrm{~nm}$. En la figura 1.5 se muestra el modo fundamental con polarización TE para una longitud de onda $\lambda=1310 \mathrm{~nm}$.
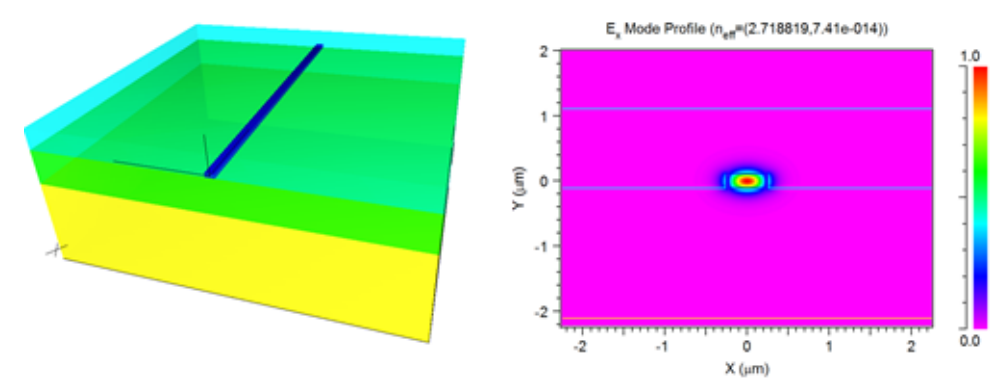

Figura 1.5: Modo fundamental TE para una guía SOI "strip" de 220nm de espesor y una anchura de 500nm.

\subsubsection{Estructuras para el acoplamiento de luz en una guía}

Uno de los grandes retos que afronta la miniaturización, refiriéndose a una guía nanofotónica, es la integración con estructuras de mayor tamaño, como una fibra óptica para inyectar o extraer luz. El área de la sección transversal de una fibra óptica $(8.2 \mu \mathrm{m}$ de 


\section{Capítulo 1: Introducción}

diámetro) es 960 veces mayor que el área de la sección transversal de una guía nanofotónica (500nm de anchura por 220nm de espesor), lo que implica grandes pérdidas al pasar la luz de una estructura a otra, tal como se observa en la figura 1.6. Por tanto, es necesaria una estructura intermedia para minimizar las pérdidas de acoplo. Varias estructuras se han propuesto y estudiado para tal efecto, que pueden dividirse en dos grupos, estructuras de acoplo en el plano o acoplo horizontal y estructuras de acoplo fuera de plano o acoplo vertical.

Las estructuras utilizadas para acoplo horizontal suelen basarse en convertidores de tamaño de modo o SSC (Spot Size Converter), mientras que las estructuras utilizadas para acoplo vertical suelen basarse en redes de difracción o "grating". Son varias las ventajas que respaldan ambas técnicas. Por un lado, el acoplamiento en el plano opera sobre un ancho de banda mucho mayor y por lo general proporcionan una mejor eficiencia de acoplo. Sin embargo, las tolerancias frente a desalineamiento suelen ser menores y no permiten el testeo a nivel de oblea. Por otro lado, el acoplamiento fuera de plano permite caracterizar los dispositivos a nivel de oblea, además de una fabricación más sencilla y una mayor tolerancia de alineamiento.

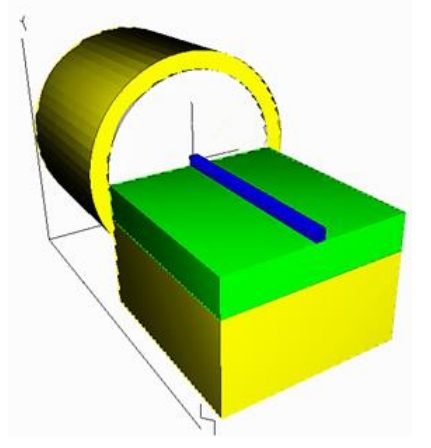

Figura 1.6: Relación de tamaño entre una guía de un PIC de espesor de $220 \mathrm{~nm}$ y ancho 500nm, respecto a una fibra óptica monomodo cuyo diámetro del núcleo es $8.2 \mu \mathrm{m}$.

\subsubsection{Acoplador mediante convertidor de tamaño de modo}

El acoplador mediante convertidor de tamaño de modo utiliza una estructura tipo cuña o "taper" invertido, que corresponde a una guía con un extremo más estrecho que se encuentra ubicado al borde del chip, tal como se ilustra en la figura 1.7. Su funcionamiento se basa en la variación del confinamiento del modo que se propaga por la guía. Así, al propagarse por el taper, el ancho del modo de propagación comienza a expandirse a medida que la guía se va estrechando cuando se supera el límite de difracción. Una capa de material con índice de refracción similar al de la fibra es esencial como cubierta del taper. Por consiguiente, la sección transversal del modo expandido será similar al modo de propagación de la fibra óptica, permitiendo operar con anchos de banda muy elevados [14].

Uno de los inconvenientes de este método radica en que las pérdidas dependen de la calidad y resolución del proceso de fabricación para definir el borde estrecho del taper, debido a que su tamaño suele ser del orden de decenas de nanómetros. No obstante, las 
pérdidas de acoplo que pueden conseguirse con este tipo de estructuras están por debajo de $1 \mathrm{~dB}$.

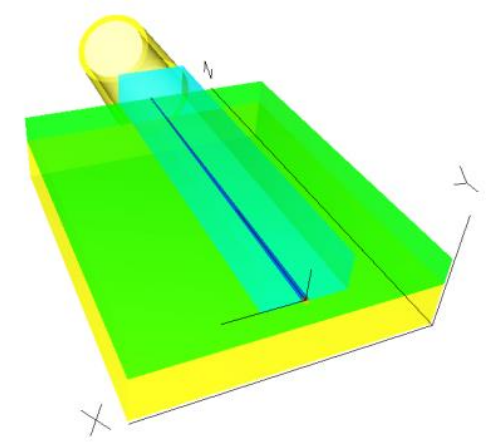

Figura 1.7: Acoplo de guía a fibra óptica mediante acoplador horizontal SSC.

Múltiples investigaciones sobre el funcionamiento y optimización de este tipo de acopladores se han realizado para longitudes de onda de operación en banda C [15]. Las estructuras propuestas para cambiar el tamaño del modo de propagación son variadas [16]. Se han demostrado pérdidas de acoplo entre $0.7 \mathrm{~dB}$ [17] y $1.5 \mathrm{~dB}$ [18] mediante convertidores de tamaño de modo basados en tapers invertidos y utilizando diversos materiales como cubierta: oxido de silicio $\left(\mathrm{SiO}_{2}\right)$ u oxinitruro de silicio ( $\mathrm{SiON}$ ), respectivamente. Otros tipos de convertidores de modo también se han evaluado y demostrado. Por un lado, se han demostrado estructuras basadas en tapers verticales con pérdidas de acoplo de $3.1 \mathrm{~dB}$ y $5.2 \mathrm{~dB}$ para polarización TM y TE respectivamente [19]. Por otro lado, también se han propuesto convertidores de tamaño de modo basados en estructuras reflectantes y anti resonantes, obteniendo pérdidas de acoplo experimentales de $4 \mathrm{~dB}$ [20]. Finalmente, se han desarrollado estructuras de taper invertido tipo tridente con pérdidas de $2.5 \mathrm{~dB}$ [21].

\subsubsection{Acoplador mediante red de difracción}

La utilización de una red de difracción es un atractivo mecanismo para acoplar luz a una guía fuera del plano. Consiste en una estructura periódica que cambia su configuración o geometría acorde a un patrón, de manera que un frente de onda que impacta sobre el mismo es perturbado y adquiere un comportamiento periódico [22]. En este sentido, el frente de onda puede ser representado a través de una serie de Fourier, donde cada componente de la onda resultante o armónico se propaga en un ángulo diferente. La onda resultante debe ser continua y uniforme con una dirección discreta determinada por los armónicos, de modo que el ángulo de las direcciones permitidas se determina por la ecuación de la red de difracción. La ecuación de la red de difracción puede ser deducida tomando como referencia los vectores de onda involucrados en el proceso. La onda incidente con vector de onda $\vec{k}_{\text {inc }}$ da lugar a una serie de ondas difractadas con vector de onda $\vec{k}_{m}$ que dependen del vector $m \vec{K}$ determinado por el periodo de la red de difracción y la constante $m$ que describe el número de armónicos permitidos $(m=0, \pm 1, \pm 2 \ldots \pm \infty)$, tal como se observa en la figura 1.8. Por lo tanto, la ecuación de la red de difracción puede ser formulada como [23]: 


\section{Capítulo 1: Introducción}

$$
\vec{k}_{m}=\vec{k}_{i n c}-m \vec{K}
$$

A partir de la Eq. 1.1 y considerando la dirección de propagación en un plano bidimensional, la condición de Bragg se deriva como:

$$
\Lambda=\frac{m \lambda}{n e f f_{g}-n_{\text {inc }} \sin \theta_{\text {inc }}}
$$

siendo $\Lambda$ el periodo de la red de difracción, $n e f f_{g}$ el índice efectivo de la guía formada en la red de difracción, $n_{i n c}$ el índice de refracción del medio de donde proviene la onda incidente y $\theta_{\text {inc }}$ el ángulo de incidencia. Sin embargo, la condición de Bragg solo permite calcular la dirección del modo difractado, pero no la cantidad de potencia de éste [24].

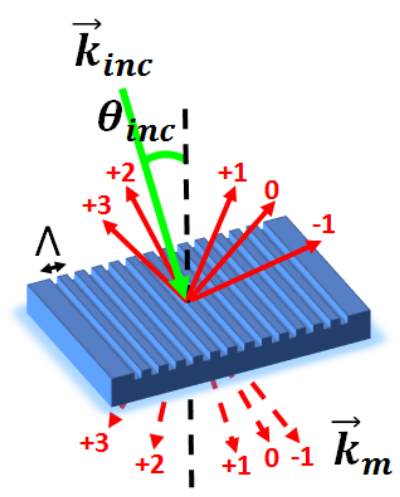

Figura 1.8: Onda incidente sobre la superficie de una estructura periódica y ondas difractadas.

Las redes de difracción para acoplar luz a una guía, tal como se ilustra en la figura 1.9, se diseñan de forma que la onda que incide sobre la superficie de la red es difractada y optimizada para acoplarse con alta eficiencia al modo de propagación de la guía, lo que hace que la red de difracción sea una estructura sensible a la polarización y con un reducido ancho de banda de operación. De acuerdo con el teorema de la reciprocidad, el mismo comportamiento ocurre cuando se acopla el modo de la guía a la fibra óptica. Las pérdidas de acoplo que pueden conseguirse con este tipo de estructuras suelen estar entre $1.5 \mathrm{~dB}$ y $5 \mathrm{~dB}$.

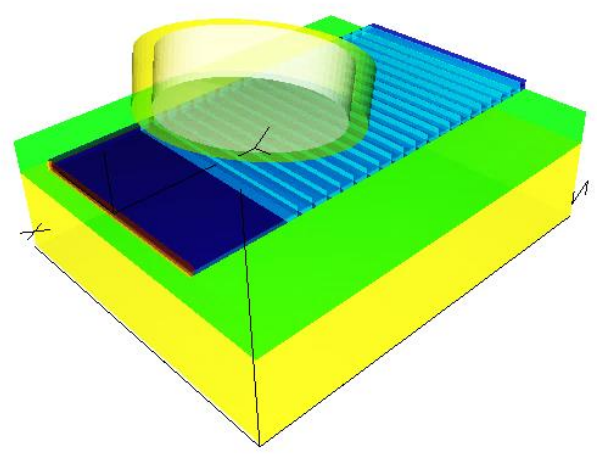

Figura 1.9: Acoplo de fibra óptica a guía mediante acoplador vertical basado en red de difracción. 
Este tipo de acopladores ha sido ampliamente investigado. Resultados experimentales muestran una eficiencia de acoplo para redes de difracción uniformes alrededor del $60 \%$ para polarización TE y del 63\% para polarización TM sobre guías de onda SOI tipo "rib" para una longitud de onda de 1550nm [25][26]. Para longitudes de onda de operación de alrededor de $1310 \mathrm{~nm}$, la eficiencia de acoplo alcanzada es de alrededor del $50 \%$ en guías de onda SOI tipo "rib" y "strip" [27]. Otros trabajos presentados reportan eficiencias de acoplo de alrededor del 40\% para longitudes de onda de operación de 1550nm [28] [29]. De igual manera, se han estudiado métodos de optimización de redes de difracción lineales. Por un lado, para redes de difracción sobre los cuales se ha aplicado apodización, la eficiencia de acoplo alcanzada es de alrededor del $60 \%$ con un ancho de banda a $3 \mathrm{~dB}$ de 64nm [30], del 40\% [31] y del 35\% [32], con un atacado completo para una longitud de onda de operación de 1550nm en polarización TE. Por otro lado, la eficiencia de acoplo alcanzada en redes de difracción sobre los cuales se ha aplicado chirp es de alrededor del $27 \%$ [33] con un atacado completo para una longitud de onda de operación de $1550 \mathrm{~nm}$, en los cuales se ha demostrado que la utilización del chirp permite incrementar el ancho de banda en una relación de 2 a 1 , siendo el ancho de banda a $3 \mathrm{~dB}$ de la red de difracción sin aplicar chirp de $32 \mathrm{~nm}$ y de $76 \mathrm{~nm}$ aplicando chirp.

\subsection{Láseres integrados sobre fotónica de silicio}

La compatibilidad con los procesos de fabricación CMOS ha dado lugar a que la tecnología fotónica de silicio se desarrolle rápidamente. Sin embargo, la integración de la fuente de luz es actualmente uno de los grandes retos pendientes. Existen tres esquemas para esta integración. El primero aborda la integración monolítica homogénea basada en silicio, aunque las soluciones estudiadas hasta el momento no han conseguido vencer la limitación del "bandgap" indirecto. El segundo consiste en una integración monolítica heterogénea mediante la integración con otros materiales, inicialmente incompatibles al proceso de fabricación, pero con mejores propiedades ópticas, para su posterior procesado y desarrollo de dispositivos emisores de luz sobre el chip. Este esquema exige una selección de materiales e incrementa significativamente la complejidad de la fabricación. El tercero y a su vez el mayor desarrollado, es la integración híbrida, escalable, de bajo coste, fiable y de mayor control térmico, que utiliza una fuente de luz o material con ganancia, es decir de tipo "bandgap" directo, fabricado de manera separada para luego ser integrado sobre el chip de silicio [34].

La integración híbrida puede llevarse a cabo mediante diferentes técnicas. Una de ellas es el encapsulado complementario, haciendo uso de micro-óptica para acoplar la luz del medio de ganancia a la guía. Otra técnica involucra el acoplamiento de borde, entre el medio de ganancia y la guía, ya sea montando dos chips lado a lado, o realizando un "flipchip bonding" de la fuente de luz sobre el chip de silicio, siendo este último método el más escalable. 


\section{Capítulo 1: Introducción}

\subsubsection{Integración monolítica sobre silicio}

Los esquemas de integración monolítica homogénea se han centrado básicamente en el desarrollo de soluciones basadas en erbio para emitir a una longitud de onda de $1550 \mathrm{~nm}$. El erbio se utiliza actualmente en la implementación de amplificadores de fibra o EDFA (Erbium-Doped Fiber Amplifier). Sin embargo, el reducido tamaño de las guías SOI y los requerimientos de bombeo eléctrico proporcionan dificultades en términos de: fabricación, coeficientes de ganancia del material y eficiencia en la transferencia de energía. No obstante, la integración monolítica de erbio dopado sobre guías SOI ha permitido demostrar una amplificación con una mejora de la señal mayor a $7 \mathrm{~dB}$ en longitudes de onda de 1530nm [35] y fuentes de luz sobre guías de onda tipo ranura o "slot" [36][37][38][39] y anillos [40].

Los esquemas de integración monolítica heterogénea se han enfocado principalmente en dos tipos de materiales: germanio (Ge) y semiconductores III-V. En el caso de germanio sobre silicio, a pesar de que ambos materiales tienen un "bandgap" indirecto, el germanio muestra un pseudo "bandgap" directo que le permite operar a una longitud de onda de 1550nm [41]. Son varios los trabajos que han demostrado la integración monolítica de germanio sobre silicio para la implementación de fuentes de luz [42][43][44]. Por otro lado, la integración de semiconductores III-V sobre silicio se ha estudiado extensamente debido a las excelentes propiedades ópticas de estos materiales además de su alta ganancia y, por tanto, mayor potencia óptica de salida. La longitud de onda de emisión dependerá del semiconductor III-V utilizado. Arseniuro de Galio (GaAs) emite en longitudes de onda entre 700nm-1.3 $\mu \mathrm{m}$, Fosfuro de Indio (InP) entre 900-2.5 $\mu \mathrm{m}$, Antimonio de Galio (GaSb) de $1.55 \mu \mathrm{m}$ al infrarrojo medio, Nitruro de Galio (GaN) para el visible y ultravioleta y Fosfuro de Galio (GaP) del visible a 1.1 $\mu \mathrm{m}$ [45]. De esta manera, se han propuesto diversas soluciones para llevar a cabo la integración [46][47][48][49][50]. La más interesante consiste en una integración monolítica basada en el crecimiento epitaxial del semiconductor sobre silicio. Sin embargo, este esquema de integración tiene una alta complejidad relacionada con los materiales. El problema principal es la hetero-epitaxia o crecimiento heterogéneo, el cual provoca una serie de defectos en la interfase entre el semiconductor III-V y el silicio, deteriorando el funcionamiento del láser.

\subsubsection{Integración híbrida sobre silicio}

La integración híbrida sobre silicio es la solución tecnológica más madura actualmente ya que permite optimizar el chip con ganancia de forma separada al chip de silicio [51]. La integración implica el acoplamiento entre ambos chips que precisa de un alineamiento con precisiones del orden de micras mediante técnicas de encapsulado avanzadas, tales como el "flip-chip bonding" mostrado en la figura 1.10. 


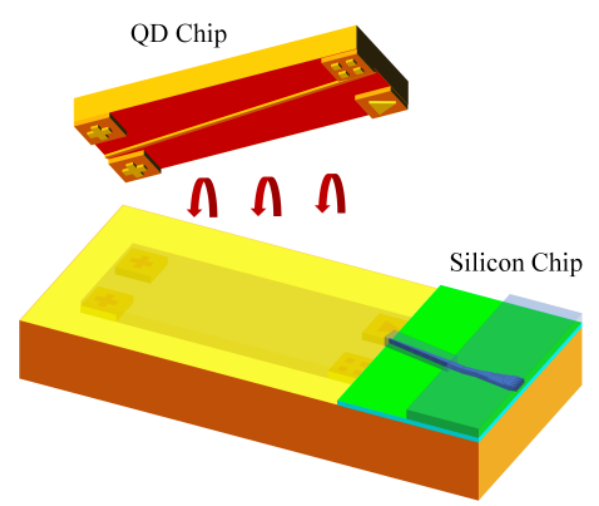

Figura 1.10: Integración híbrida de una fuente láser sobre un chip SOI, por medio de la técnica "flip-chip bonding".

De esta forma es posible implementar láseres de cavidad externa o ECDL (External Cavity Diode Laser). La cavidad externa se realiza en el chip de silicio y consta en su configuración más sencilla de un reflector distribuido de Bragg o DBR (Distributed Bragg Reflector). De esta manera se da retorno de la luz al medio de ganancia, produciendo la amplificación óptica mediante emisión estimulada. Sin embargo, es imprescindible emitir a una sola longitud de onda, por lo cual la posibilidad de selección de la longitud de onda dentro de la cavidad es necesaria para conseguir un láser monomodo.

Respecto al chip con ganancia o medio activo a utilizar, trabajos previos en el diseño de fuentes de luz han demostrado que la utilización de dispositivos de bajas dimensiones con un confinamiento cuántico en el área activa favorece la superposición entre electrones y huecos, repercutiendo en el funcionamiento del láser a diferencia de utilizar el material base. De este modo la mayoría de los dispositivos láser publicados utilizan pozos cuánticos QW (Quantum Wells) o puntos cuánticos QD (Quantum Dot) como nanoestructuras activas. Los pozos cuánticos permiten una modificación de las bandas de energía del semiconductor. En este caso, los electrones y huecos se confinan dentro de la capa de emisión, de manera que, si el pozo cuántico es lo suficientemente delgado, el confinamiento cuántico ocurre y se discretizan los niveles de energía, permitiendo sintonizar la banda prohibida y por tanto la longitud de onda de emisión [52]. Los puntos cuánticos o QDs van más allá permitiendo un confinamiento cuántico en las tres dimensiones o 3D. Los QDs suelen tener un ancho típico de unas pocas docenas de nanómetros y altura de unos pocos nanómetros. De esta manera se alcanza un confinamiento 3D sobre los electrones y huecos, lo que permite una recombinación eficiente y una verdadera discretización de los niveles de energía, de manera que las transiciones ópticas dentro del material son atómicas. El tamaño y composición del QD rige los niveles de energía por medio de un confinamiento cuántico directo y por lo tanto la longitud de onda de emisión del láser [53]. Otra de las mejoras de los QDs respecto a los QWs es la disminución de la densidad de corriente límite para llegar al modo de emisión estimulada, la operación a altas temperaturas y una mayor potencia de salida. [54] 


\section{Capítulo 1: Introducción}

\subsubsection{Estado del arte de láseres con cavidad externa}

La integración híbrida se ha llevado a cabo con diversas técnicas. Una de ellas es la técnica conocida como "wafer bonding" [55][56][60], que consiste en la inserción de los elementos activos sobre la oblea de silicio. Otra técnica es el acoplamiento lateral o de borde "butt-coupling" [64] de tipo no invasiva ya que mantiene la independencia de ambas partes. Y por último, la técnica "flip-chip bonding" [57][58][59][61][62][63][65] de tipo invasiva, ya que el medio activo es depositado y alineado sobre el chip de silicio. Esta tesis se basa en el desarrollo de un láser que combina las dos últimas técnicas: acoplamiento lateral entre el chip activo, colocado mediante "flip-chip" en el chip de silicio, y la cavidad externa implementada en el mismo chip de silicio.

El desarrollo de un láser de cavidad externa basado en la integración de una fuente de luz de banda ancha sobre un chip de silicio que integra una cavidad externa requiere, por un lado, un dispositivo reflector y, por otro lado, una estructura de acoplo, para acoplar de manera eficiente la luz emitida por el medio activo. En 2008 y 2013 se propuso la implementación de una fuente láser basada en dos estructuras DBR (Distributed Bragg Reflector) junto con un anillo resonante [55][56][60].La cavidad del láser estaba formada por el medio de ganancia basado en un semiconductor del grupo III-V, integrado al chip de silicio mediante "wafer bonding" y acoplado a la guía de silicio mediante un convertidor de modo adiabático. Finalmente, un calentador metálico sobre un anillo resonante permitía la sintonización de la longitud de onda del láser. Investigaciones recientes también han demostrado la implementación de una fuente láser sintonizable con integración híbrida y acoplamiento lateral, haciendo uso de convertidores de tamaño de modo para proporcionar una adaptación modal entre la guía de la cavidad del chip de silicio y el chip activo [57][58][61][62][63][64][65][66]. De la misma manera para la sintonización se utilizó micro-calentadores sobre anillos resonantes. Un acoplamiento con bajas pérdidas entre el chip de silicio y el medio activo es clave para optimizar las prestaciones del láser. Por lo cual, se han propuesto estructuras de acoplo basadas en guías en forma de tridente con bajas pérdidas de acoplo y un amplio ancho de banda de operación, que permite una alta densidad de operación multicanal, a través de una integración híbrida con "flip-chip bonding" [62][63]. La Tabla 1.1 muestra un resumen del estado del arte de desarrollos de láseres bajo un esquema de integración híbrida en la banda de operaciones C y O. Además, en la Tabla 1.2 se muestran las especificaciones de operación de cada uno de los láseres de cavidad externa. 
1.3 Láseres integrados sobre fotónica de silicio

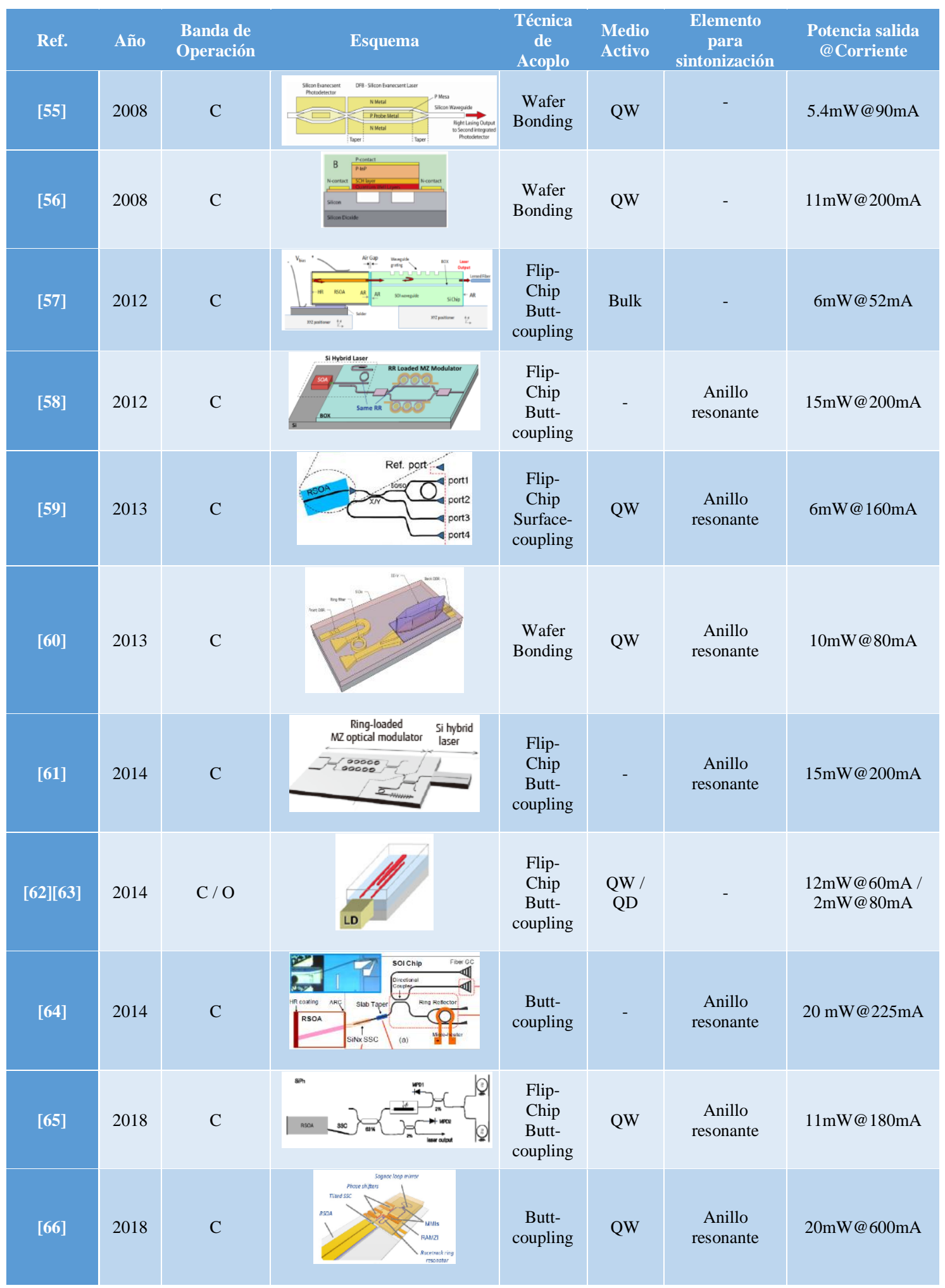

Tabla 1.1: Estado del arte de integración híbrida sobre Si, para el desarrollo de láseres de cavidad externa, se detalla en cada caso la banda de operación $(C=1550 \mathrm{~nm}, \mathrm{O}=1310 \mathrm{~nm})$, la técnica de acoplo y/o tecnología utilizada para la integración, el tipo de dispositivo activo, la estructura utilizada para sintonización y la potencia de salida alcanzada del láser con la respectiva corriente inyectada en el dispositivo activo. 


\section{Capítulo 1: Introducción}

\begin{tabular}{|c|c|c|c|c|c|}
\hline Ref. & $\begin{array}{c}\text { Corriente } \\
\text { umbral }\end{array}$ & $\begin{array}{l}\text { Ancho } \\
\text { de línea }\end{array}$ & SMSR & WPE & $\begin{array}{l}\text { Rango de } \\
\text { Temperatura }\end{array}$ \\
\hline [55] & $25 \mathrm{~mA}$ & $3.6 \mathrm{MHz}$ & $50 \mathrm{~dB}$ & - & $10^{\circ} \mathrm{C}-50^{\circ} \mathrm{C}$ \\
\hline [56] & $65 \mathrm{~mA}$ & - & $50 \mathrm{~dB}$ & - & $15^{\circ} \mathrm{C}-45^{\circ} \mathrm{C}$ \\
\hline [57] & $\begin{array}{l}13 \mathrm{~mA}- \\
15.3 \mathrm{~mA}\end{array}$ & - & $45 \mathrm{~dB}$ & $9.5 \%$ & - \\
\hline [58] & $9.4 \mathrm{~mA}$ & & $40 \mathrm{~dB}$ & $7.6 \%$ & $20^{\circ} \mathrm{C}-60^{\circ} \mathrm{C}$ \\
\hline [59] & $43 \mathrm{~mA}$ & - & $30 \mathrm{~dB}$ & $4.5 \%$ & - \\
\hline [60] & $65 \mathrm{~mA}$ & $1.7 \mathrm{MHz}$ & $45 \mathrm{~dB}$ & - & $20^{\circ}$ \\
\hline [61] & $9.8 \mathrm{~mA}$ & - & $38 \mathrm{~dB}$ & - & $25^{\circ} \mathrm{C}-60^{\circ} \mathrm{C}$ \\
\hline$[62][63]$ & $11 \mathrm{~mA}$ & - & - & - & $25^{\circ} \mathrm{C}-100^{\circ} \mathrm{C}$ \\
\hline [64] & $39 \mathrm{~mA}$ & $27 \mathrm{MHz}$ & $35 \mathrm{~dB}$ & $7 \%$ & - \\
\hline [65] & $30 \mathrm{~mA}$ & $37 \mathrm{KHz}$ & $55 \mathrm{~dB}$ & $4.2 \%$ & $25^{\circ} \mathrm{C}$ \\
\hline [66] & $95 \mathrm{~mA}$ & $30 \mathrm{MHz}$ & $35 \mathrm{~dB}$ & $5 \%$ & \\
\hline
\end{tabular}

Tabla 1.2: Especificaciones de operación de los láseres de cavidad externa presentados, se detalla el umbral de corriente sobre la cual el láser se encuentra en estado de emisión estimulada, el ancho de línea o ancho de banda a $3 \mathrm{~dB}$ de la salida del láser, la relación de supresión de modo lateral SMSR (Side Mode Suppression Ratio), la eficiencia de radiación WPE (Wall-plug Efficiency) y el rango de temperatura de operación del láser.

\subsection{Objetivos y organización de la tesis}

Este trabajo pretende contribuir al desarrollo de dispositivos emisores de luz para sistemas de transmisión óptica de alta velocidad. El objetivo principal será la implementación de un láser de cavidad externa formado por una fuente de luz de banda ancha integrada híbridamente con una cavidad externa en tecnología fotónica de silicio. Además, se abordará también el acoplamiento a estructuras de guiado tipo ranura, las cuales han sido propuestas en esquemas de integración monolítica. De esta forma, los objetivos específicos de la tesis han sido:

- Desarrollo de estructuras de acoplamiento a fibra óptica basadas tanto en red de difracción como basadas en convertidores de tamaño de modo.

- Desarrollo de reflectores basados en red de difracción.

- Desarrollo de estructuras de filtrado y sintonización termo-óptica.

- Diseño, integración y demostración experimental de un láser de cavidad externa basado en las estructuras previamente desarrolladas.

La longitud de onda de funcionamiento del láser estará en el rango de la banda $\mathrm{O}$ (1260nm-1360nm), para aplicaciones futuras en el campo de las comunicaciones de datos 
(datacom) y en sistemas de comunicaciones basados en multiplexación de longitud de onda (WDM), lo que supone un reto tanto a nivel de diseño como a nivel de fabricación.

El contenido de la tesis está estructurado en cinco capítulos. El primer capítulo proporciona una breve introducción sobre la tecnología fotónica de silicio, en la cual se resaltan los beneficios de esta tecnología, así como también las oportunidades, retos e inconvenientes para el desarrollo de nuevos dispositivos de comunicaciones. De esta forma, se han introducido diversos conceptos partiendo de las propiedades ópticas del silicio, las guías SOI y las técnicas de acoplamiento a fibra óptica. Además, se han planteado las bases de funcionamiento de láseres basados en integración monolítica y láseres de cavidad externa basados en integración híbrida sobre fotónica de silicio. Finalmente, se ha realizado una revisión del estado del arte de los trabajos más recientes en esta área.

El capítulo 2 aborda el diseño de un acoplador de red difracción sobre guías de onda tipo ranura o "slot", con la cual se alcanza un alto confinamiento de la luz en una pequeña sección transversal de la guía. Este tipo de guías ha sido propuesto para la implementación de láseres basados en esquemas de integración monolítica.

El capítulo 3 contiene el diseño, modelado y simulación de un láser de cavidad externa basado en integración híbrida. Por un lado, se ha diseñado mediante simulación las diferentes estructuras necesarias. El método de simulación de elementos finitos o FEM (Finite Elements Method) se ha utilizado para evaluar la guía y determinar los modos de propagación en la misma. El método de propagación de haz o BPM (Beam Propagation Method) y el método de diferencias finitas en el dominio del tiempo o FDTD (Finite Diferencias Time Domain) se han usado para evaluar las estructuras de acoplo, red de difracción y convertidores de tamaño de modo, así como también los anillos resonantes y reflectores de Bragg. Por otro lado, se ha realizado un trabajo de modelado para determinar en una primera instancia las dimensiones del modo de propagación de la fuente de luz utilizada en la integración, partiendo de las especificaciones técnicas de la misma. De la misma manera, se ha realizado un modelo aproximado del funcionamiento de la cavidad externa, integrando todos los bloques de diseño que la componen. Con el cual, se ha evaluado la tolerancia respecto a las pérdidas soportadas en la cavidad.

El capítulo 4 describe los resultados experimentales más relevantes obtenidos en cada una de las etapas del desarrollo del láser basado en integración híbrida. La caracterización de las distintas estructuras, tales como acopladores, anillos resonantes o reflectores, se aborda en primer lugar. Finalmente, se demuestra el funcionamiento del láser, como dispositivo final que integra todos los bloques de diseño.

Por último, en el capítulo 5 se resumen las conclusiones alcanzadas y se proponen las líneas de trabajo futuro. 



\section{Capítulo 2}

\section{Acoplador de luz a fibra para esquemas de integración monolítica}

\subsection{Descripción de guía tipo ranura considerada}

La integración de alta densidad para aplicaciones de interconexión óptica es uno de los grandes objetivos de la fotónica de silicio. De esta manera, la posibilidad de integración vertical de múltiples capas con componentes fotónicos es un esquema prometedor frente a la integración limitada a una sola capa [67]. Además, dicho esquema permite también la combinación de capas ópticas y electrónicas [68][69][70][71][72]. La fabricación de circuitos integrados multicapa es posible mediante el depósito de capas de distintos materiales o la unión directa de obleas mediante técnicas de "wafer bonding”. En las guías tipo ranura o "slot", el confinamiento de la luz se produce en una delgada capa de material con bajo índice de refracción que se encuentra entre dos materiales más gruesos de índice de refracción mayor. Este tipo de guías ha sido propuesto para la implementación de láseres basados en esquemas de integración monolítica [35]-[40] y por tanto un aspecto crucial es el desarrollo de estructuras de acoplamiento eficientes a fibra óptica.

En la figura 2.1(a) se muestra una guía "slot" horizontal en la cual se consigue un alto confinamiento de luz en la delgada capa de bajo índice de refracción para la polarización TM. Por el contrario, en una guía "slot" vertical, el alto confinamiento de luz en la capa de bajo índice de refracción se obtiene para la polarización TE, tal y como se observa en la figura 2.1(b). 
Capítulo 2: Acoplador de luz a fibra para esquemas de integración monolítica
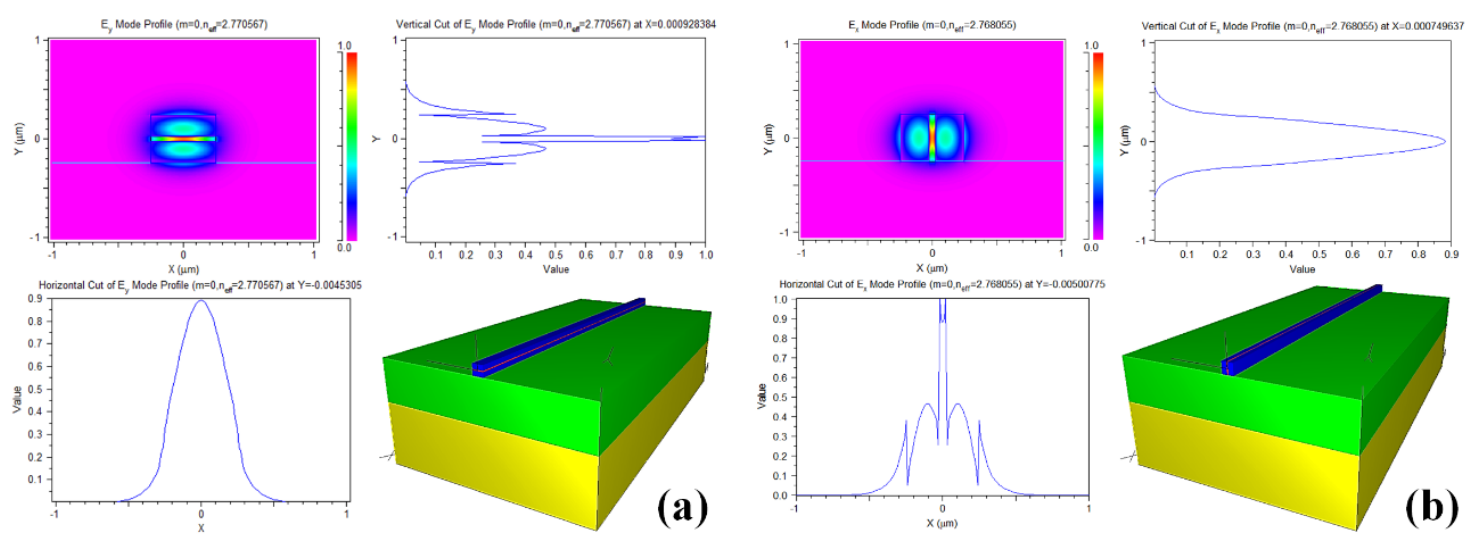

Figura 2.1: Guía tipo ranura y modo de propagación fundamental en guía "slot": (a) Horizontal y polarización TM, (b) Vertical y polarización TE.

La guía tipo ranura considerada es una guía "slot" horizontal y se muestra en la figura 2.2. El material con bajo índice de refracción situado en la ranura o "slot" es nitruro de silicio $\left(\mathrm{Si}_{3} \mathrm{~N}_{4}\right)$ con un índice de refracción $n_{\text {slot }}=1.989$, que se encuentra sobre dos capas de silicio $(\mathrm{Si})$ con índice de refracción $n_{s i}=3.476$. La guía está recubierta de óxido. En la figura 2.2 se detallan los principales parámetros de diseño correspondientes al espesor de cada una de las capas que conforman la guía. El ancho de guía empleado es de $\mathrm{Wg}_{\text {width }}=300 \mathrm{~nm}$ para asegurar una transmisión monomodo.

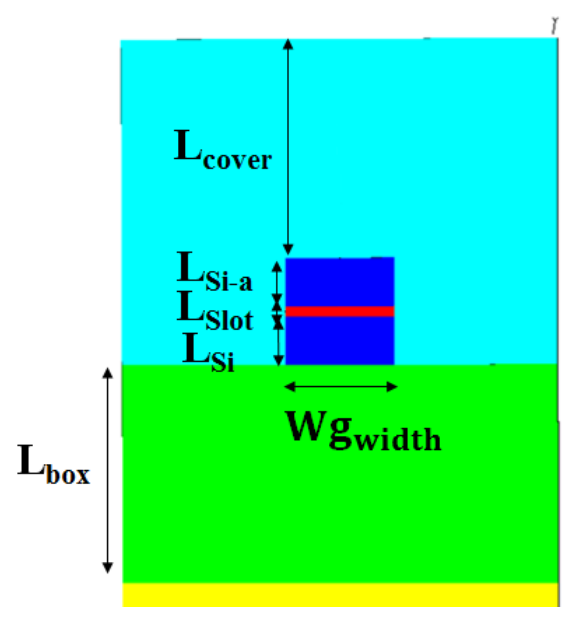

Figura 2.2: Guía "slot" horizontal que se utilizó para el diseño del acoplador a fibra óptica.

El objetivo es desarrollar una estructura de acoplamiento basada en red de difracción y que no exija un paso de ataque adicional para simplificar así la fabricación. La utilización de un nivel de atacado total o "full-etched" en redes de difracción para acoplamiento a guías convencionales SOI exige la optimización de estructuras más avanzadas para maximizar la eficiencia de acoplo [73][74][75][76][77]. No obstante, en el caso de guías tipo ranura se han demostrado estructuras de acoplo basadas en redes de difracción convencionales con eficiencias de acoplo de hasta el 60\% [78] [79] [80]. 


\subsection{Diseño del acoplador a fibra basado en red de difracción}

El diseño de la estructura de acoplo basada en red de difracción o grating se ha llevado a cabo para una longitud de onda $\lambda=1550 \mathrm{~nm}$ y polarización TM. La guía multicapa ha sido detallada en la sección anterior. La implementación de la misma se realiza a partir de una oblea SOI comercial y, por tanto, con una capa de silicio de espesor $L_{S i}=220 \mathrm{~nm}$ y una capa de óxido $L_{B o x}=2 \mu \mathrm{m}$. La capa de nitruro de silicio de espesor $L_{S l o t}$ se deposita a continuación y sobre ésta la capa de silicio amorfo de espesor $\mathrm{L}_{\mathrm{Si}-\mathrm{a}}$. La estructura de acoplo puede ser diseñada en un plano bidimensional sobre un corte longitudinal de la red de difracción, asumiendo un ancho de guía infinito, debido a que éste es mucho mayor que la longitud de onda del modo de propagación [81]. El diseño de la red de difracción se basa en una estructura atacada hasta la capa de óxido o "full-etched". En la figura 2.3 se muestra el esquema de la red de difracción con todos los parámetros de diseño.

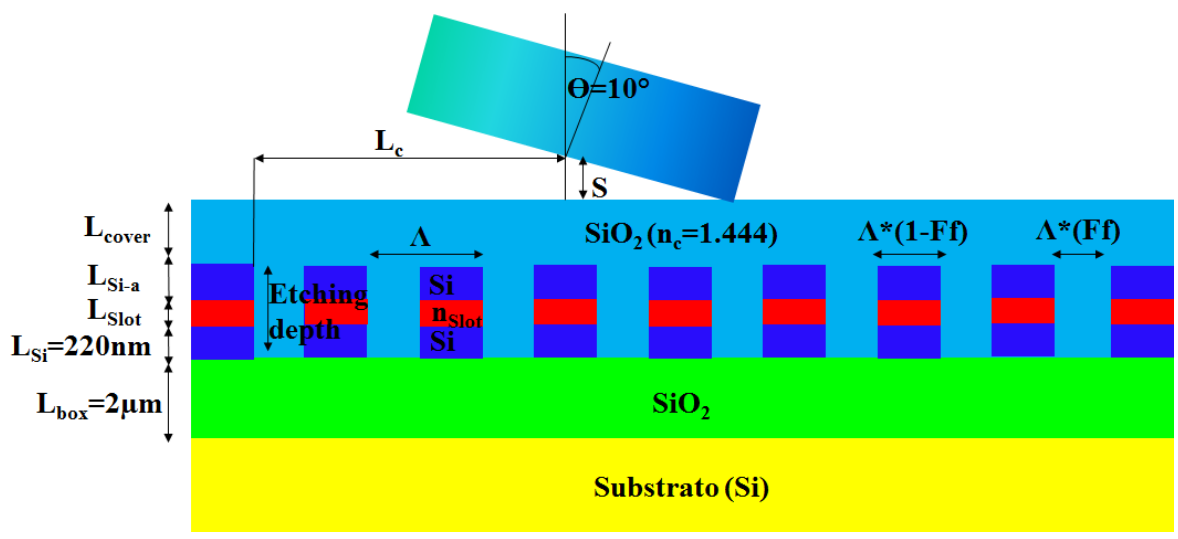

Figura 2.3: Corte longitudinal de la estructura de acoplo sobre guía "slot", en la cual se detalla: Parámetros de diseño de la guía referentes al espesor de las capas de: óxido $\left(L_{c o v e r}, L_{b o x}\right)$, silicio $\left(L_{S i}, L_{S i-a}\right)$, nitruro de silicio $\left(L_{S l o t}\right)$. Parámetros de la red de difracción: periodo de la red de difracción $(\Lambda)$, factor de llenado ( $F f$ ), profundidad de atacado (Etching depth). Parámetros de alineamiento de la fibra y la red de difracción: ángulo de incidencia $\left(\theta=10^{\circ}\right)$, longitud de acoplo $\left(L_{c}\right)$ y distancia de la fibra a la superficie de la red de difracción $(S)$.

La fibra óptica con la cual se acopla luz a la guía se ubica sobre la superficie de la red de difracción, con un ángulo de incidencia $\theta=10^{\circ}$, y a una distancia respecto al inicio de la red de difracción $L c$, cuyo valor se obtiene mediante

$$
L c=\frac{w_{0}}{1.37 * \operatorname{Cos} \Theta}
$$

siendo $w_{o}$ la mitad del diámetro del campo modal MFD=10.4 $\mu \mathrm{m}$ (Modal Field Diameter) del haz gaussiano incidente correspondiente al modo fundamental de una fibra monomodo estándar a una longitud de onda $\lambda=1550 \mathrm{~nm}$ [82].

El diseño se ha realizado mediante el análisis de dos casos: (i) una estructura con las dos capas de silicio simétricas en términos de espesor y (ii) una estructura con ambas capas asimétricas. En ambos casos, la variación del espesor de la capa de nitruro de silicio también se analizó y se llevó a cabo la optimización del periodo $\Lambda$ y factor de llenado $F f$. En una primera etapa se ajustaron varios parámetros de alineamiento en relación con la 
posición de la fibra óptica respecto a la red de difracción, valores que se mantuvieron en todo el estudio, siendo $S=1 \mu m$ y $L_{c}=3.5 \mu m$. Después, se analizó el efecto del espesor de la capa de óxido que cubre la red de difracción, observándose una respuesta sinusoidal respecto a la eficiencia de acoplo, por lo cual se ajustó un valor de $L_{\text {cover }}=1 \mu \mathrm{m}$ que corresponde al punto máximo de eficiencia de acoplamiento encontrado. De esta manera, para cada variación de los parámetros de la guía "slot" se recalculó el valor del periodo $\Lambda$ con un valor inicial para el factor de llenado de $F f=50 \%$.

El diseño se llevó a cabo mediante simulaciones 2D-FDTD. Inicialmente se evaluó el caso de guía "slot" simétrica $\left(L_{S i}=L_{S i-a}\right)$. Se realizó una variación del espesor de las capas de la guía, determinando el valor óptimo para la capa de nitruro de silicio $L_{S l o t}=$ $50 \mathrm{~nm}$ y para las capas de silicio $L_{S i}=L_{S i-a}=235 \mathrm{~nm}$ (figura 2.4(a)). Con los valores óptimos de las capas de la guía, se realizó una variación del periodo $\Lambda$ y factor de llenado $F f$, siendo los óptimos $\Lambda=895 \mathrm{~nm}$ y $F f=52 \%$ respectivamente (figura $2.4(\mathrm{~b})$ ). En este caso se observó que en polarización TM el periodo es más susceptible a variaciones que el factor de llenado. De esta manera, manteniendo el valor óptimo del espesor de la capa de nitruro de silicio $L_{S l o t}=50 \mathrm{~nm}$ y del factor de llenado $F f=52 \%$, se realizó nuevamente una variación del periodo y las capas de silicio (figura 2.4(c)), alcanzando una eficiencia de acoplo de alrededor del $44 \%$ para un valor de periodo $\Lambda=880 \mathrm{~nm}$ y de las capas de silicio de $L_{S i}=L_{S i-a}=245 \mathrm{~nm}$.
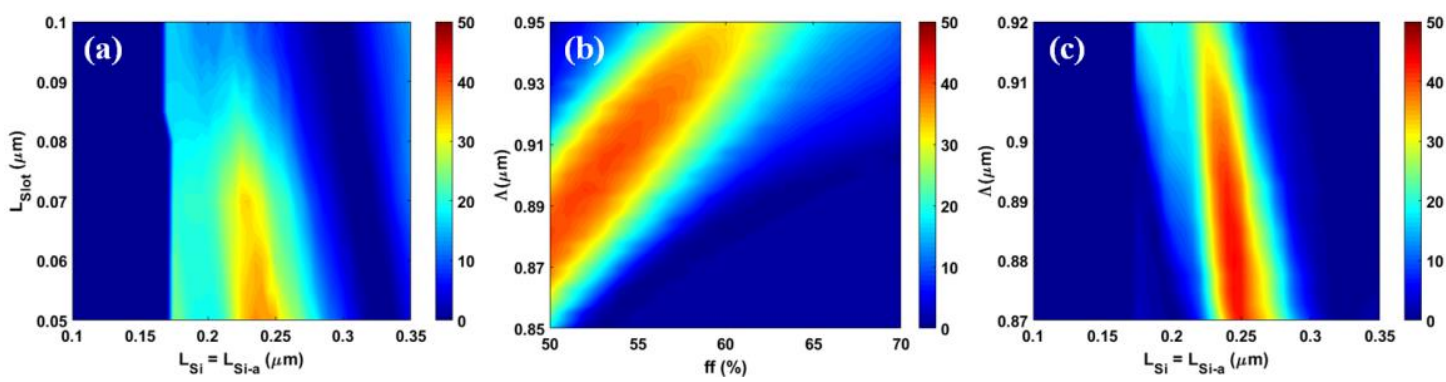

Figura 2.4: Simulaciones 2D-FDTD con resolución 30nm de la eficiencia de acoplo en función de: (a) Espesor de la capa de nitruro de silicio y de las capas de silicio, (b) Periodo y factor de llenado, y (c) Periodo y espesor de las capas de silicio.

Para el caso de guía "slot" asimétrica $\left(L_{S i} \neq L_{S i-a}\right)$, se fijó el espesor de la capa de silicio en el valor estándar de una oblea SOI, $L_{S i}=220 \mathrm{~nm}$, y se realizó una variación del espesor la capa de silicio amorfo y nitruro de silicio, siendo óptimos los valores $L_{S i-a}=$ $285 \mathrm{~nm}$ y $L_{\text {Slot }}=50 \mathrm{~nm}$ (figura $2.5(\mathrm{a})$ ). Con los valores óptimos de espesor de las capas de silicio amorfo y nitruro de silicio, se llevó a cabo una variación del periodo y factor de llenado, de tal manera que los valores óptimos en este caso fueron de $\Lambda=895 \mathrm{~nm}$ y $\mathrm{Ff}=$ $56 \%$ respectivamente (figura $2.5(\mathrm{~b})$ ). Finalmente, se realizó nuevamente una variación del periodo y espesor de la capa de silicio amorfo, determinando los valores óptimos en $\Lambda=895 \mathrm{~nm}$ y $L_{S i-a}=295 \mathrm{~nm}$ respectivamente, y alcanzando una eficiencia de acoplo de alrededor del 52\% mayor que el caso de guía tipo "slot" simétrica (figura 2.5(c)), de este modo este diseño fue utilizado para su implementación. Los parámetros óptimos de la 
estructura de acoplo diseñada tanto sobre una geometría de guía "slot" simétrica y asimétrica se describen en la tabla 2.1.
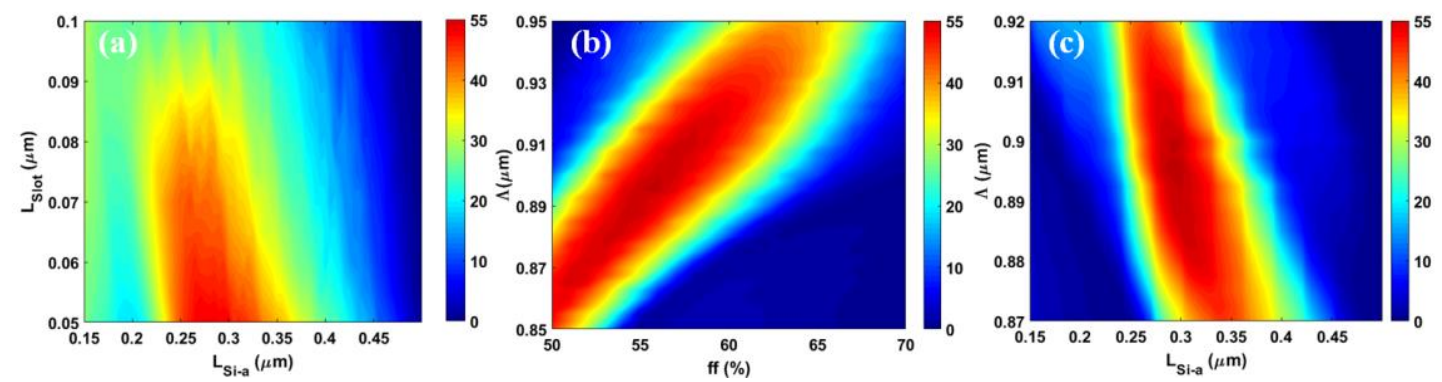

Figura 2.5: Simulaciones 2D-FDTD con resolución 30nm de la eficiencia de acoplo en función de: (a) Espesor de la capa de nitruro de silicio y de las capas de silicio, (b) Periodo y factor de llenado, y (c) Periodo y espesor de las capas de silicio.

\begin{tabular}{|c|c|c|}
\hline Parámetros de diseño & $\begin{array}{c}\text { Diseño óptimo guía } \\
\text { simétrica }\end{array}$ & $\begin{array}{c}\text { Diseño óptimo guía } \\
\text { asimétrica }\end{array}$ \\
\hline Espesor de la cubierta de óxido & $1 \mu \mathrm{m}$ & $1 \mu \mathrm{m}$ \\
\hline Espesor de la capa de silicio amorfo & $245 \mathrm{~nm}$ & $295 \mathrm{~nm}$ \\
\hline Espesor de la capa de nitruro de silicio & $50 \mathrm{~nm}$ & $50 \mathrm{~nm}$ \\
\hline Espesor de la capa de silicio & $245 \mathrm{~nm}$ & $220 \mathrm{~nm}$ \\
\hline Periodo de la red de difracción & $880 \mathrm{~nm}$ & $895 \mathrm{~nm}$ \\
\hline Factor de llenado & $52 \%$ & $56 \%$ \\
\hline
\end{tabular}

Tabla 2.1: Parámetros de diseño óptimos del acoplador a fibra óptica por medio de red de difracción sobre una guía tipo ranura simétrica y asimétrica.

La anchura de la red de difracción diseñada debe ser equiparable al diámetro del núcleo de la fibra óptica, cuyo valor estándar es de $12 \mu \mathrm{m}$. Sin embargo, se requiere acoplar luz a una guía de ancho 300nm, por lo cual se necesita una estructura tipo cuña o "taper" posterior a la red de difracción que conecte la estructura de acoplo con la guía nanofotónica (figura 2.6 (a)). Para evitar el uso de estos tapers, se usan estructuras de acoplo formadas a partir de elipses confocales, que permiten un acoplo directo a la guía y reducir así de manera considerable el tamaño final de la estructura. Los parámetros de diseño mostrados en la tabla 2.1 fueron utilizados en el diseño de la red de difracción de elipses confocales, en la cual se mantiene un foco común y se construye cada elipse en función del periodo y factor de llenado óptimos obtenidos en el diseño original. Así, se generaron las partes atacadas y no atacadas de la red de difracción, de las cuales se extrajo un área en forma de taper, cuyo extremo estrecho corresponde al ancho de la guía y en la cual se encuentra el punto focal común y que se extiende hasta la última elipse confocal en su parte más ancha de $12 \mu \mathrm{m}$ (figura 2.6 (b)). Las elipses confocales se diseñaron, a partir de la condición de Bragg para el primer orden de difracción, por medio de la siguiente expresión [83]:

$$
\sqrt{x^{2}+z^{2}} k_{\text {in }} n_{\text {eff }}-z k_{\text {in }} n_{\text {cover }} \sin (\theta)=2 \pi q
$$

correspondiendo el plano $(x, z)$ a la vista superior de la red de difracción, el eje $z$ a la distancia respecto al punto focal común, $k_{\text {in }}$ el vector de onda incidente, $n_{\text {cover }}$ el índice de refracción del material que cubre la estructura, $n_{e f f}$ el índice efectivo de propagación 


\section{Capítulo 2: Acoplador de luz a fibra para esquemas de integración monolítica}

en la guía y $q$ un entero que indica el orden de la red de difracción curvada. Utilizando la Eq.2.2 se generó la siguiente expresión que permite describir un conjunto de elipses confocales con un punto focal común en el origen:

$$
\frac{\left(z-\frac{q \lambda_{0} n_{\text {cover } \sin (\theta)}}{n_{\text {eff }}^{2}-n_{\text {cover }}^{2} \sin (\theta)^{2}}\right)^{2}}{\left(\frac{q \lambda_{0} n_{\text {eff }}}{n_{\text {eff }}^{2} n_{\text {cover }}^{2} \sin (\theta)^{2}}\right)^{2}}+\frac{x^{2}}{\left(\frac{q \lambda_{0}}{\sqrt{n_{\text {eff }}^{2}-n_{\text {cover }}^{2} \sin (\theta)^{2}}}\right)^{2}}=1
$$

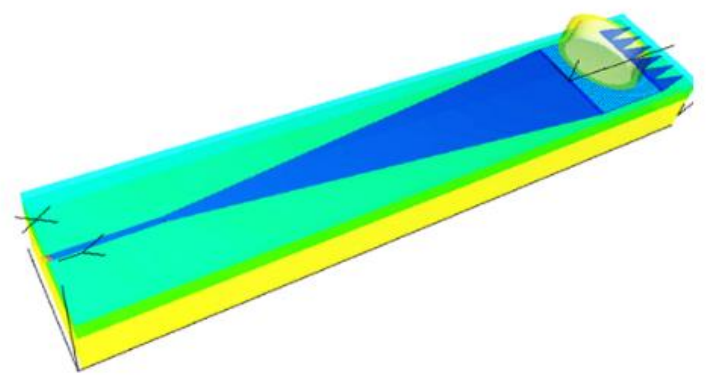

(a)

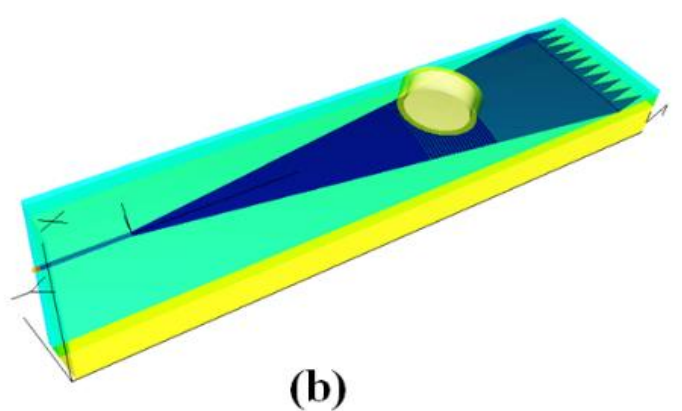

(b)

Figura 2.6: Acoplador a fibra óptica basado en red de difracción: (a) Red de difracción lineal con estructura "taper" para conectar la guía del acoplador con la guía, (b) Red de difracción curvada a partir de elipses confocales, estructura que conecta directamente el acoplador a la guía.

El espectro de las estructuras de acoplo diseñadas se analizaron por medio de varias simulaciones. Por un lado, se realizaron simulaciones 3D-FDTD con resolución 50nm para evaluar la red de difracción curvada a partir de elipses confocales. Por otro lado, se llevaron a cabo simulaciones 2D-FDTD con resolución 30nm para evaluar la red de difracción lineal sin taper. En ambos casos el espectro resultante se encuentra centrado entorno a $\lambda=1550 \mathrm{~nm}$ y tiene una máxima eficiencia de acoplamiento de alrededor del $48 \%$ para la red de difracción curvada y de $52 \%$ para la red de difracción lineal, diferencia atribuida a la menor resolución utilizada en la simulación 3D-FDTD. Por otra parte, el ancho de banda a $3 \mathrm{~dB}$ es de $73 \mathrm{~nm}$ y $77 \mathrm{~nm}$ respectivamente, tal como se observa en la figura 2.7 .

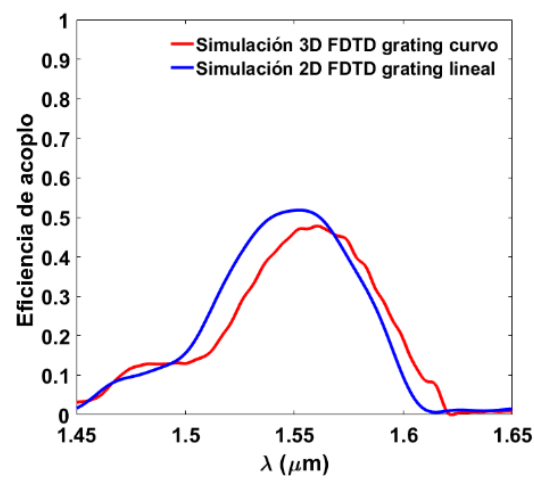

Figura 2.7: Eficiencia de acoplo frente a longitud de onda de las estructuras de acoplo diseñadas basadas en red de difracción curvada y lineal. 


\subsection{Fabricación y resultados experimentales}

La capa de nitruro de silicio, $\mathrm{Si}_{3} \mathrm{~N}_{4}$, con un espesor de $50 \mathrm{~nm}$ se depositó por medio de la técnica PECVD (Plasma-Enhanced Chemical Vapor Deposition) sobre la capa de silicio cristalino de la oblea SOI. Posteriormente, una capa de silicio amorfo, a-Si, con el valor óptimo de espesor de $295 \mathrm{~nm}$ se depositó utilizando la misma técnica sobre la capa de nitruro de silicio. Los índices de refracción medidos por elipsometría en cada caso para una longitud de onda de $\lambda=1550 \mathrm{~nm}$ fueron de 1.96 y 3.41 respectivamente, muy parecidos a los utilizados en simulación. El proceso de fabricación se basó en un proceso de grabado directo mediante un haz de electrones (e-beam) sobre una cubierta de $180 \mathrm{~nm}$ de espesor correspondiente a una película resistiva de hidrógeno silsesquioxane (HSQ). La exposición del haz de electrones se desarrolló con el equipo Vistec EBG5000, que se optimizó para alcanzar las dimensiones requeridas empleando un voltaje de aceleración de $100 \mathrm{KeV}$ y un tamaño de apertura de $200 \mu \mathrm{m}$. Después de grabar el diseño en la resina HSQ, utilizando hidróxido de tetrametilamonio como revelador, el patrón de la resina se transfirió a la muestra de silicio amorfo empleando un proceso optimizado reactivo de grabado de iones con plasma acoplado inductivamente (ICP-RIE) con gases de fluoruro. Finalmente, se depositó una cubierta de óxido de espesor $1 \mu \mathrm{m}$ sobre la muestra mediante PECVD. Posteriormente a la fabricación se realizaron imágenes SEM de la estructura de acoplo fabricada mediante red de difracción curvada, utilizando elipses confocales (figura 2.8(a)). En la figura 2.8(b) se observa la muestra con varias guías de diferentes longitudes para caracterizar las pérdidas de propagación, y finalmente, la figura 2.8(c) muestra el corte transversal de la guía en la cual se observan todas las capas de la guía "slot" diseñada.
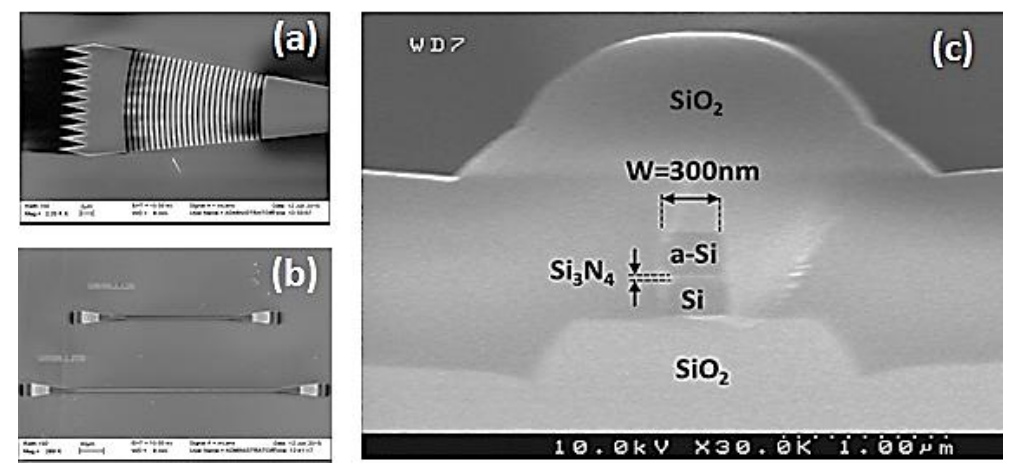

Figura 2.8: Imágenes SEM de: (a) Acoplador a fibra mediante red de difracción, (b) Muestra con varias estructuras de acoplo en guías de diferentes longitudes, y (c) Corte transversal de la guía multicapa "slot" fabricada.

La caracterización de la muestra se realizó con un montaje de acoplo vertical. La fibra óptica se colocó a un ángulo de inclinación de $10^{\circ}$ respecto a la normal de la superficie de la red de difracción. Mediante una fuente de luz sintonizable externa (SANTEC TSL210F) conectada a un polarizador manual se ajustó la polarización TM, y éste a su vez se conectó a la fibra óptica de entrada, con la cual se inyectó la luz sobre la red de difracción. Con otra fibra en el puerto de salida, conectada a un multímetro óptico (Thorlabs PM320E), se detectó la potencia óptica de luz acoplada a la fibra. Una vez se alinearon las fibras por medio de unidades 


\section{Capítulo 2: Acoplador de luz a fibra para esquemas de integración monolítica}

de traslación en tres ejes, se obtuvo el espectro de funcionamiento en guías con diferentes longitudes haciendo un barrido a nivel de longitud de onda con el láser sintonizable (figura 2.9(a, b)). Se observó que el espectro de funcionamiento se encontraba desplazado a la longitud de onda de $\lambda=1480 \mathrm{~nm}$ respecto a la longitud de onda de diseño. Este desplazamiento se debe a una variación durante el proceso de fabricación de los parámetros tanto de la guía "slot" como de la red de difracción respecto a los valores de diseño. Por lo cual, se llevó a cabo nuevas simulaciones con los parámetros de la estructura fabricada para comprobar el desplazamiento y validar el diseño. En la tabla 2.2 se describen los parámetros óptimos de diseño y los alcanzados en fabricación.
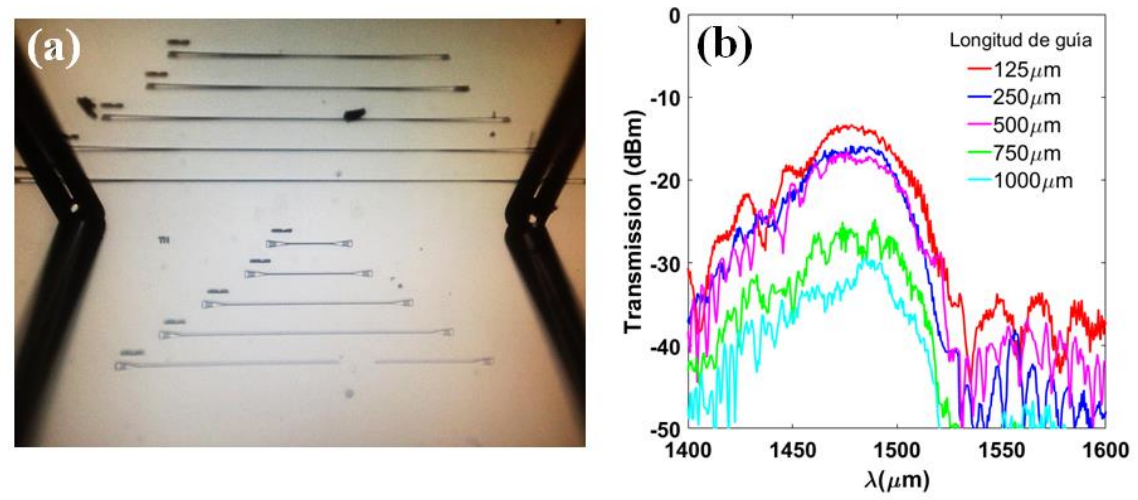

Figura 2.9: (a) Imágenes de la muestra con estructuras de acoplo a fibra en guías de varias longitudes. (b) Espectro de transmisión medido en cada estructura de guía con diferente longitud.

\begin{tabular}{|c|c|c|}
\hline Parámetros de diseño & Óptimo & Medido \\
\hline Espesor de la cubierta de óxido & $1 \mu \mathrm{m}$ & $1 \mu \mathrm{m}$ \\
\hline Espesor de la capa de silicio amorfo & $295 \mathrm{~nm}$ & $300 \mathrm{~nm}$ \\
\hline Espesor de la capa de nitruro de silicio & $50 \mathrm{~nm}$ & $40 \mathrm{~nm}$ \\
\hline Espesor de la capa de silicio & $220 \mathrm{~nm}$ & $220 \mathrm{~nm}$ \\
\hline Periodo de la red de difracción & $895 \mathrm{~nm}$ & $902 \mathrm{~nm}$ \\
\hline Factor de llenado & $56 \%$ & $69 \%$ \\
\hline
\end{tabular}

Tabla 2.2: Parámetros de diseño óptimos y medidos después de fabricación de la estructura de acoplo a fibra sobre guía "slot".

Las pérdidas de acoplo se calcularon con:

$$
\alpha_{g}[d B]=\frac{1}{2}\left(P_{\text {in }}[d B m]-P_{\text {out }}[d B m]-\alpha_{w g} L_{w g}[d B]-\alpha_{\text {setup }}[d B]\right)
$$

siendo $P_{\text {in }}$ y $P_{\text {out }}$ las potencias de entrada y salida, $\alpha_{w g}$ y $L_{w g}$ las pérdidas de propagación y longitud de las guías y $\alpha_{\text {setup }}$ las pérdidas del montaje experimental. Las pérdidas de propagación se estimaron utilizando el método de "cut-back" en $\alpha_{w g} @ 1480 \mathrm{~nm}=$ $85 \mathrm{~dB} / \mathrm{cm}$. El alto valor se atribuye principalmente a la variabilidad de la selectividad en el ataque debido a los distintos materiales involucrados que origina variaciones en la sección la transversal. Las pérdidas del montaje debido a conectores se establecieron en $\alpha_{\text {setup }}=1.4 \mathrm{~dB}$. En la figura $2.10 \mathrm{se}$ muestra el espectro normalizado de la estructura de acoplo y el espectro simulado con los valores de los parámetros de la estructura medidos después de fabricación. Las pérdidas de acoplo alcanzadas, calculadas a partir de la Eq. 
2.4, son de $\alpha_{g}=4.4 d B$, muy similar al valor obtenido en simulación. El ancho de banda medido a $3 \mathrm{~dB}$ del espectro resultante es de $38 \mathrm{~nm}$.

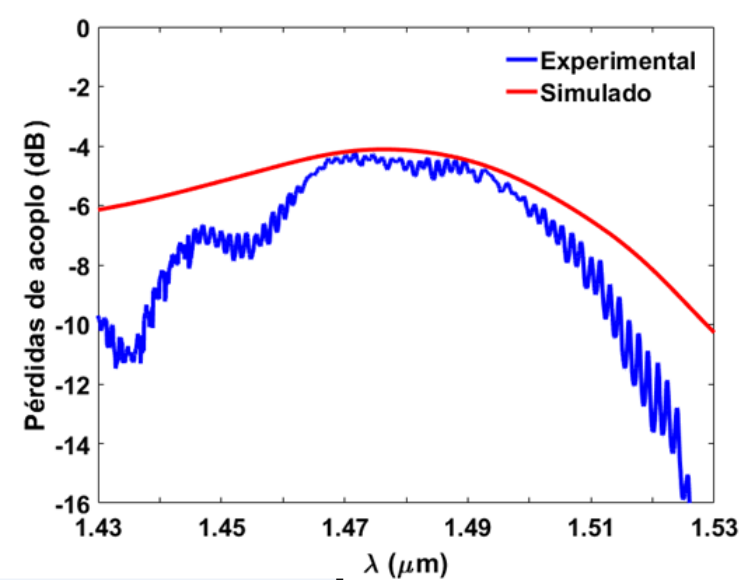

Figura 2.10: Espectro de funcionamiento del acoplador a fibra obtenido experimentalmente y en simulación con los parámetros de la estructura medidos después de fabricación. 



\section{Capítulo 3}

\section{Diseño esquema de integración híbrida de láser con cavidad externa}

\subsection{Descripción del esquema propuesto}

El objetivo principal de esta tesis doctoral es la implementación de un láser monomodo de cavidad externa con funcionamiento en la banda de $1310 \mathrm{~nm}$. El láser propuesto está formado por la integración híbrida entre una fuente de luz de banda ancha, de un material semiconductor del grupo III-V que constituye el medio de ganancia, y el chip de silicio, compuesto por varias estructuras nanofotónicas, dando lugar a un láser de cavidad externa o ECDL. Dicho dispositivo es clave en sistemas de transmisión óptica de alta velocidad y permitiría interconexiones ópticas incluso a nivel de chip. En la figura 3.1 se muestra el esquema del dispositivo propuesto. La cara altamente reflectiva de la fuente de luz ancha o QD y el reflector DBR situado al final de la guía de silicio forma una cavidad Fabry-Perot. Un aspecto clave para el correcto funcionamiento del láser son las bajas pérdidas de acoplo entre ambos chips, que se llevará a cabo por medio de una adaptación modal entre ambas interfaces, haciendo uso de un convertidor de tamaño de modo o SSC que se ubicará al borde del chip de silicio. La utilización de SSCs requiere de una alta precisión a nivel de alineamiento que asegure unas bajas pérdidas de acoplo por lo que en una primera fase la integración híbrida se llevará a cabo a través de un alineamiento activo y posteriormente mediante la técnica de "flip-chip bonding" y por tanto un alineamiento pasivo. Además, la salida de potencia del ECDL será extraída de manera eficiente del chip de silicio por medio de otro SSC o red de difracción para acoplamiento con una fibra 
óptica de salida. Para asegurar un funcionamiento monomodo, se empleará un filtro basado en anillo resonante dentro de la cavidad, además de incorporar micro calentadores o "heaters" sobre dicho anillo resonante y sobre una sección de la guía, para maximizar la potencia de salida del láser y permitir una sintonización de la longitud de onda de emisión.

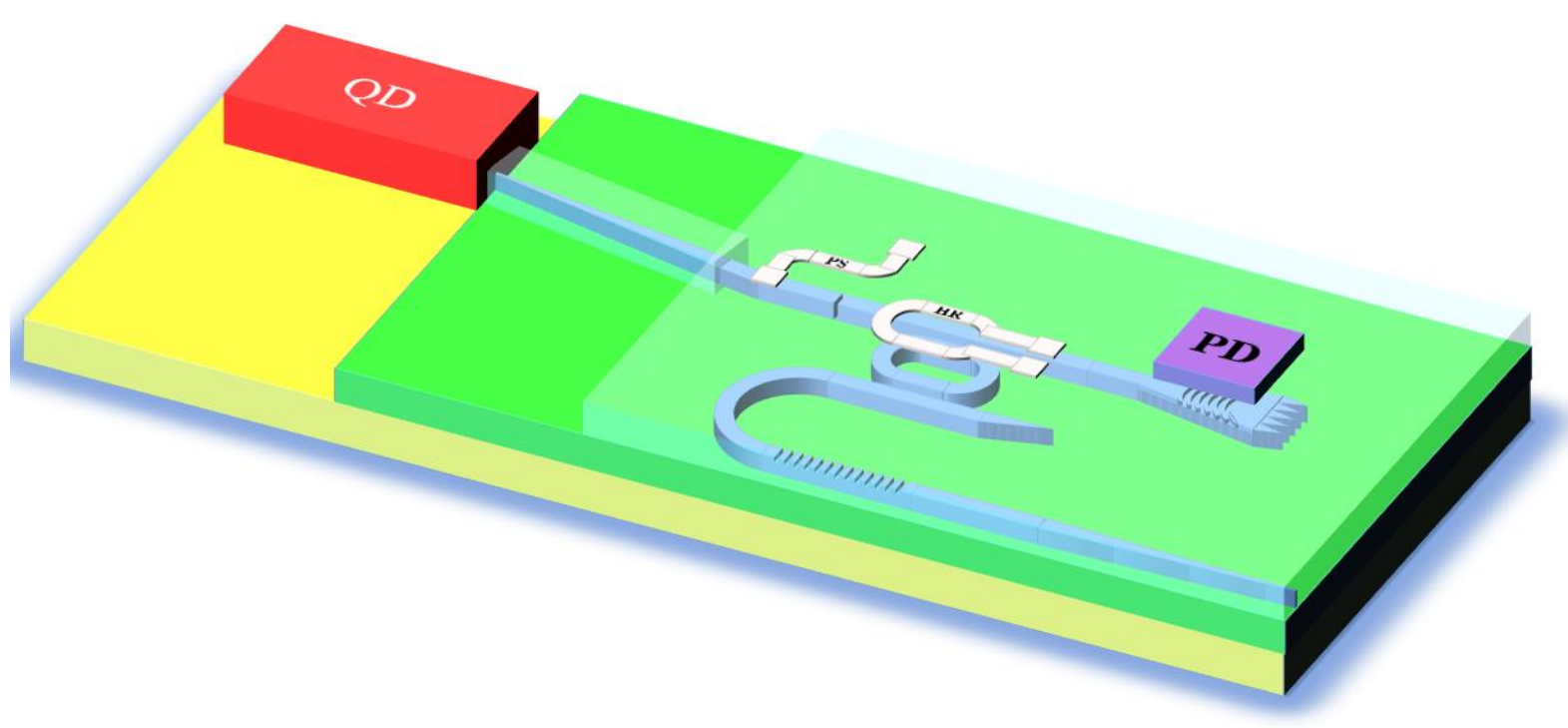

Figura 3.1: Esquema del láser de cavidad externa propuesto basado en la integración híbrida de una fuente de luz ancha o QD en un chip de silicio. Se detalla los micro calentadores sobre el anillo resonante HR (Heater Ring) y sobre la guía PS (Phase Shifter), además del fotodetector PD (Photodetector) para función de monitoreo en una de las salidas del filtro de anillo resonante.

\subsection{Diseño del acoplador a láser basado en convertidor de tamaño de modo}

El convertidor de tamaño de modo elegido se basa en una estructura de cuña o taper invertido. En el caso de acoplo de una guía nanofotónica en el chip de silicio a otra guía en el chip de ganancia o QD, la estructura de taper invertido se forma mediante una reducción progresiva desde la parte ancha del taper hasta su parte estrecha, produciendo que el modo de propagación que proviene de la guía de silicio se expanda progresivamente llegando a tener un tamaño equiparable al modo emitido por el QD y por tanto acoplándose eficientemente a éste. De igual manera, para el caso de acoplo del QD a la guía de silicio, el factor de confinamiento de la guía va incrementándose desde su parte estrecha hasta la parte ancha del taper, produciendo que el modo de propagación que proviene del QD se reduzca hasta quedar confinado y acoplado al modo de la guía de silicio. En la figura 3.2 se observan los parámetros del acoplador para su diseño, para lo cual se han tenido en cuenta las tres dimensiones de la estructura. 


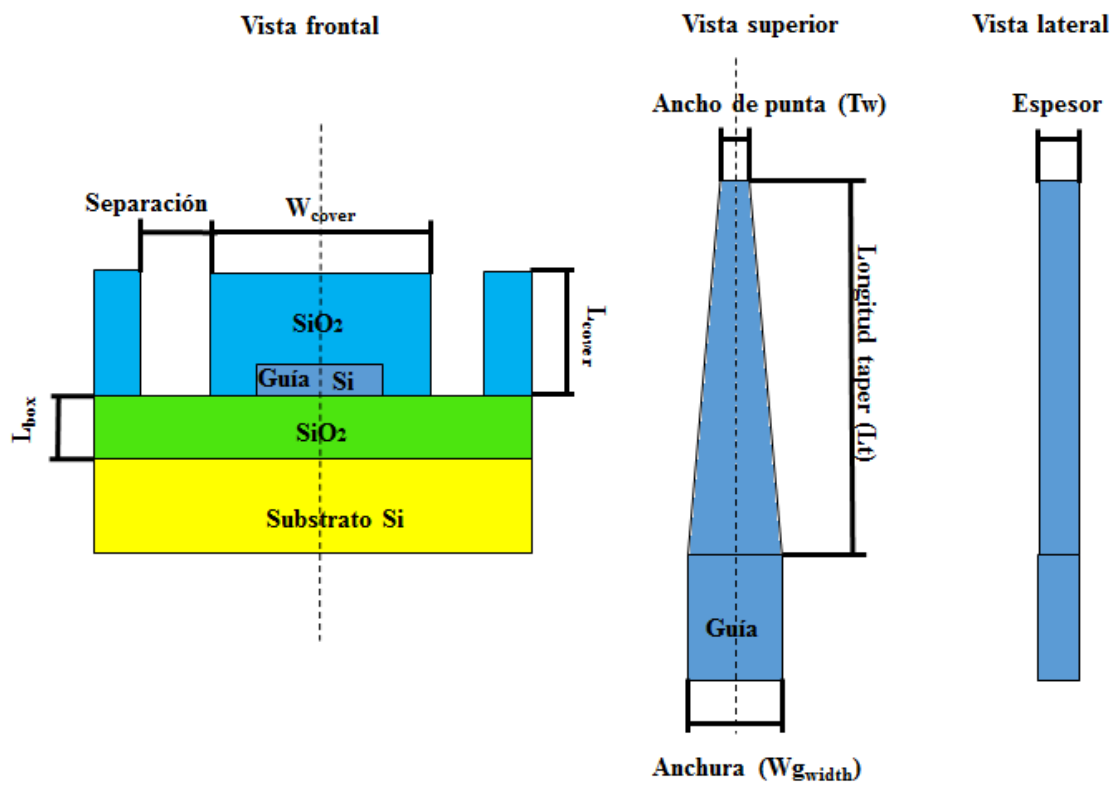

Figura 3.2: Parámetros de diseño del acoplador a fibra óptica basado en un convertidor de tamaño de modo. Se detalla las dimensiones del taper invertido: longitud $(L t)$, ancho de punta $(T w)$, anchura de guía $\left(W g_{\text {width }}=500 \mathrm{~nm}\right)$, espesor de la guía $(220 \mathrm{~nm})$, y las dimensiones de la cubierta del acoplador: ancho de cubierta $\left(W_{\text {cover }}\right)$, espesor de las capas de óxido $\left(L_{b o x}, L_{c o v e r}\right)$.

\subsubsection{Especificaciones del QD}

El acoplo entre la fuente de luz o QD (figura 3.3) y la guía de silicio es esencial para la implementación del láser. Para el diseño del convertidor de tamaño de modo es necesario modelar el modo de propagación del haz de luz de emisión del QD, de tal manera que los parámetros del acoplador sean óptimos respecto al perfil modal de emisión del QD en el campo cercano (near field). Para el modelado del modo de propagación del haz de luz del QD se utilizaron las especificaciones técnicas del mismo, que se detallan en la tabla 3.1.

\begin{tabular}{|c|c|c|}
\hline $\begin{array}{c}\text { Ancho de } \\
\text { banda } \\
(\mathbf{n m})\end{array}$ & $\begin{array}{c}\text { Ángulo de divergencia } \\
\text { del haz en el eje lento } \\
\text { FWHM }\left(^{\circ}\right)\end{array}$ & $\begin{array}{c}\text { Ángulo de divergencia } \\
\text { del haz en el eje rápido } \\
\text { FWHIM }\left(^{\circ}\right)\end{array}$ \\
\hline 35 & 10 & 61 \\
\hline
\end{tabular}

Tabla 3.1: Especificaciones de operación del QD: ancho de funcionamiento del espectro a $3 \mathrm{~dB}$ (Spectral width) y ángulos de divergencia a la anchura completa a mitad del máximo (FWHM) en campo lejano (Far field) del haz de luz emitido, correspondientes al eje lento (Slow axis) y eje rápido (Fast axis). 


\section{Capítulo 3: Diseño esquema de integración híbrida de láser con cavidad externa}

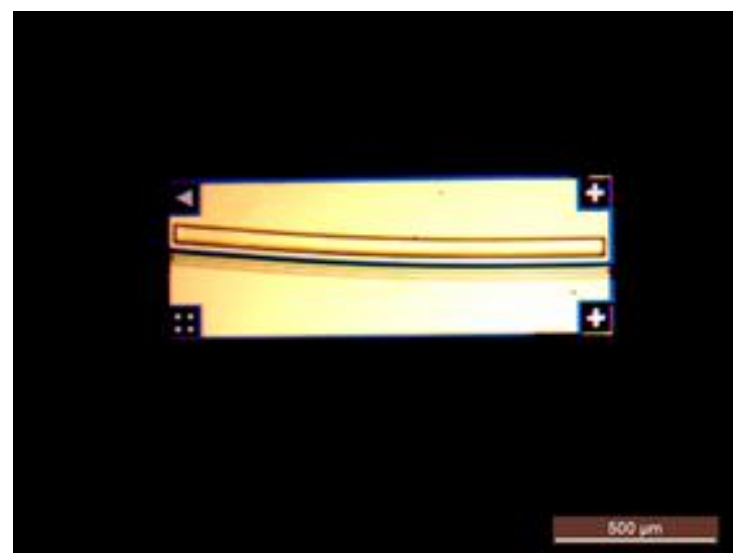

Figura 3.3: Imagen del chip de ganancia QD empleado para la implementación del láser de integración híbrida. Se observa que la guía con respecto al punto de emisión tiene un ligero ángulo de inclinación de $5^{\circ}$ para evitar reflexiones indeseadas.

Los ángulos de divergencia del modo de emisión del láser proporcionados por el fabricante son en campo lejano (far field). Por ello, el paso previo para el diseño del convertidor de tamaño de modo es modelar el perfil modal del haz de emisión del láser en campo cercano (figura 3.4).

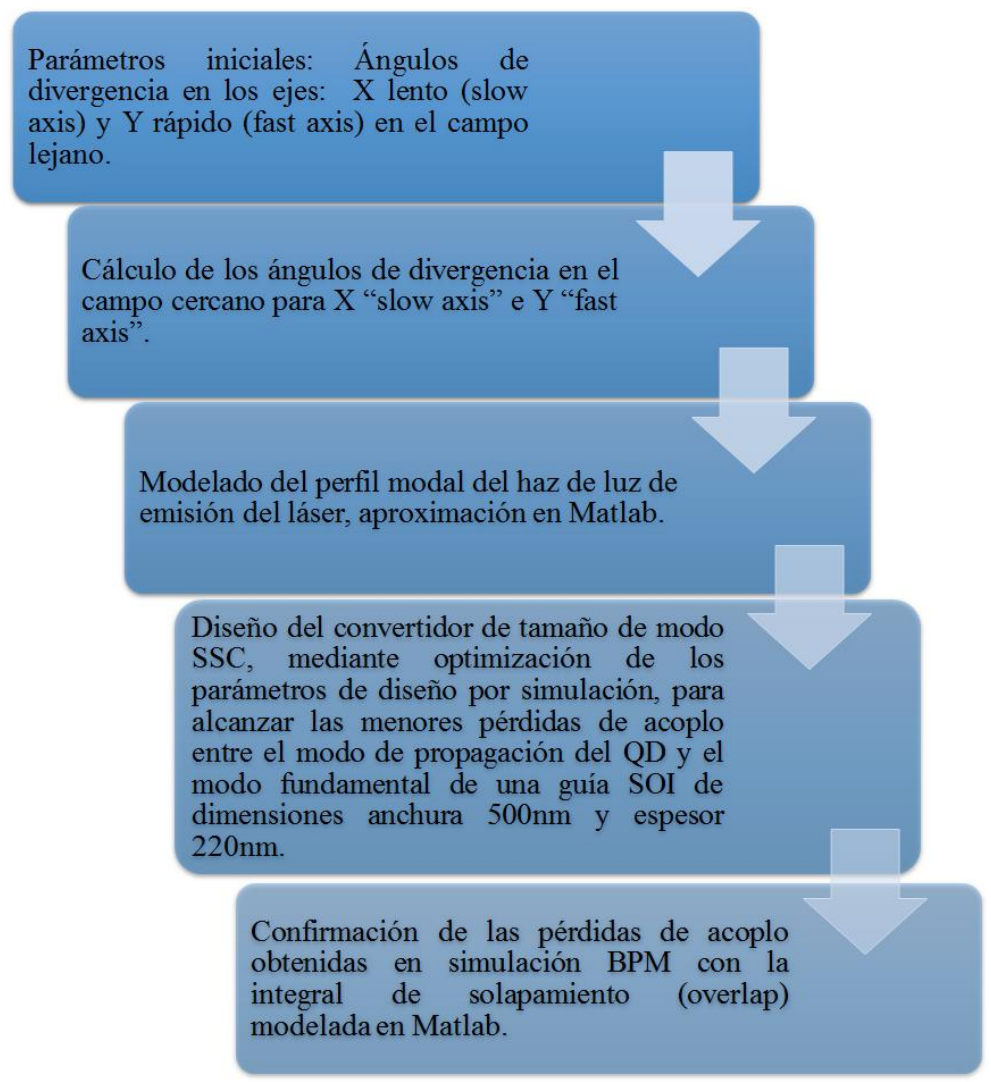

Figura 3.4: Flujo de proceso seguidos para el diseño del convertidor de tamaño de modo para acoplo entre un chip QD y una guía de silicio.

El cálculo de las dimensiones del modo de emisión del láser en campo cercano se realizó mediante la expresión de factor de calidad (Eq. 3.1) de una onda de luz que se 
propaga en el espacio, y cuyo valor se aproxima a $M^{2}=1$ para un haz gaussiano, siendo $2 w_{o}$ el diámetro del haz en campo cercano, $\theta$ el ángulo de divergencia en radianes (Eq. 3.2) y $\lambda$ la longitud de onda del modo de propagación [84].

$$
\begin{aligned}
& M^{2}=\frac{2 w_{o} \pi \theta}{4 \lambda} \rightarrow M^{2}=1 \Rightarrow 2 w_{o}=\frac{4 \lambda}{\pi \theta} \\
& 2 \theta=\frac{\sqrt{2}}{\sqrt{\ln 2}} \mathrm{FWHM}(\mathrm{rad}) \rightarrow \theta=\frac{\mathrm{FWHM}}{\sqrt{2 \ln 2}}
\end{aligned}
$$

De esta forma, para los ángulos de divergencia en campo lejano detallados en la tabla 3.1 , las dimensiones del haz obtenidas en campo cercano corresponden a $5.623 \mu \mathrm{m}\left(2 w_{o x}\right)$ en el eje lento y $0.922 \mu \mathrm{m}\left(2 w_{o y}\right)$ en el eje rápido, tal como se muestra en la figura 3.5.

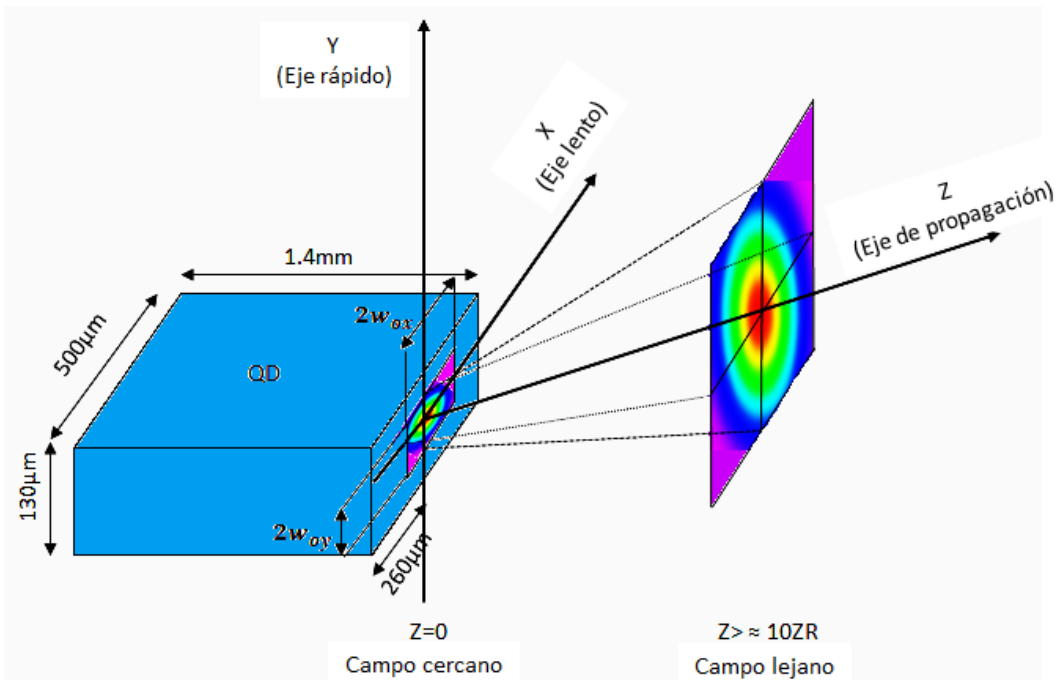

Figura 3.5: Esquema de propagación del haz de luz de emisión del QD y diferencia de tamaño de modo en campo cercano y lejano, debido a los ángulos de divergencia en los ejes lento y rápido.

Con los valores obtenidos del diámetro del haz de luz emitido por el QD en campo cercano, se modeló el perfil del modo mediante la utilización de la ecuación de onda paraxial y su solución en el plano bidimensional en coordenadas rectangulares (Eq. 3.3), [85][86]. En dicha ecuación, $\left(w_{o x}, w_{o y}\right)$ representa la anchura del haz, $\left(R_{x}, R_{y}\right)$ corresponde al radio de curvatura del haz gaussiano y $\left(\emptyset_{\mathrm{ox}}, \emptyset_{\mathrm{oy}}\right)$ representa la fase de la onda en el eje $x$ e $y$, respectivamente para cada caso.

$$
E(x, y, z)=\left(\frac{2}{\pi w_{o x} w_{o y}}\right)^{0.5} \cdot \exp \left(-\frac{x^{2}}{w_{o x}{ }^{2}}-\frac{y^{2}}{w_{o y^{2}}}-\frac{j \pi x^{2}}{\lambda R_{x}{ }^{2}}-\frac{j \pi y^{2}}{\lambda R_{y}{ }^{2}}+\frac{j \emptyset_{o x}}{2}+\frac{j \emptyset_{o y}}{2}\right)
$$

En la figura 3.6 se muestra un esquema de la propagación del haz de luz emitido y la variación del diámetro en función de la distancia de propagación. Asimismo, se detalla la ecuación para el cálculo de la distancia Rayleigh o ZR a la cual el diámetro del haz es máximo. 


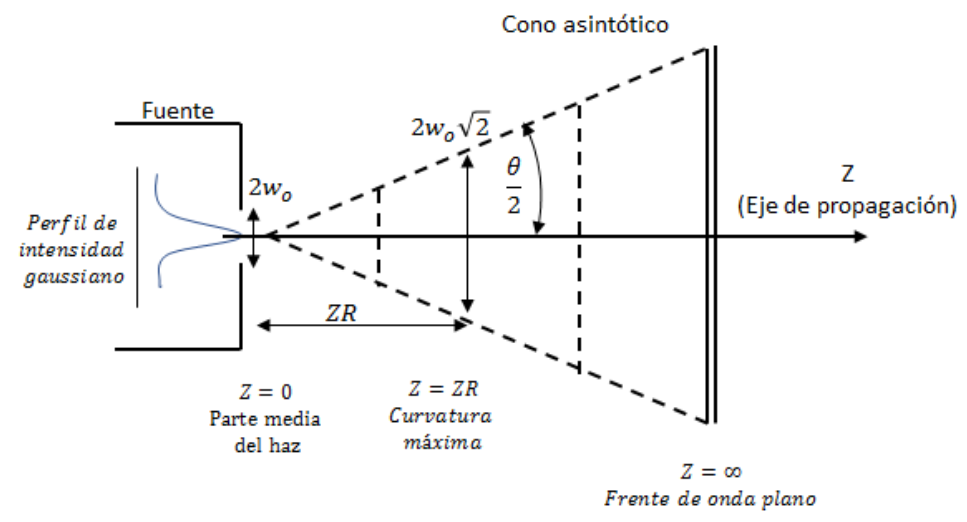

Figura 3.6: Esquema de propagación del haz de luz de emisión del QD en el vacío y la evolución del diámetro de la cintura de modo en función de la distancia de propagación.

En la figura 3.7 se muestra el perfil del modo de propagación modelado en Matlab en campo cercano, así como el diámetro del haz en cada uno de los ejes $(x, y)$ correspondiente a la longitud para la cual la intensidad del haz de luz decae a $1 / e^{2}(13.5 \%$ del pico).
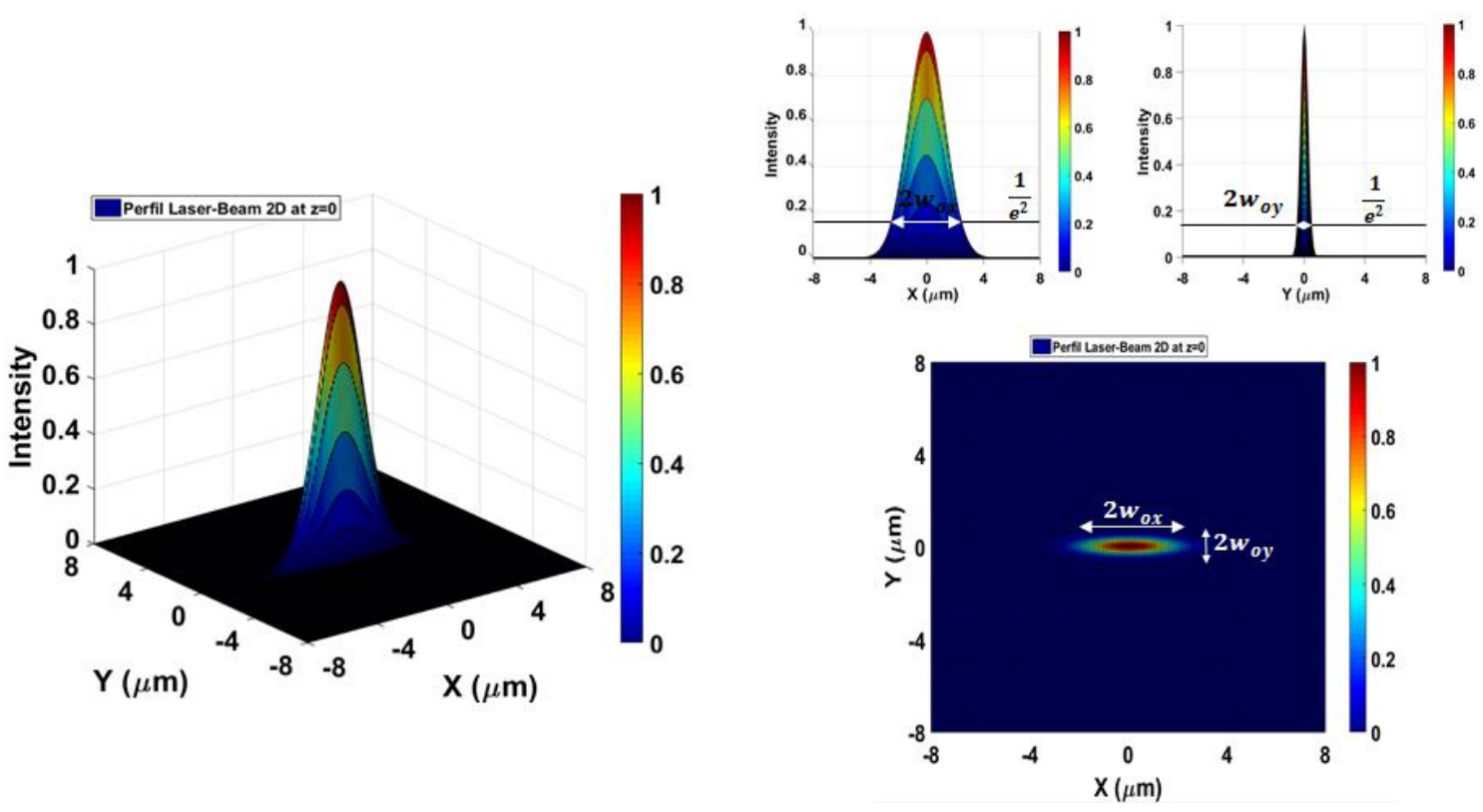

Figura 3.7: Modelo de propagación del haz de luz emitido por el QD, en el campo cercano, en el cual el diámetro del modo fundamental gaussiano a $1 / e^{2}$ es de $2 \mathrm{w}_{\mathrm{ox}}=5.623 \mu \mathrm{m}$ y $2 \mathrm{w}_{\mathrm{oy}}=0.922 \mu \mathrm{m}$, en el eje $\mathrm{x}$ e y respectivamente.

Después del modelado del modo de propagación de la fuente de luz QD, se llevaron a cabo simulaciones 3D-BPM para una longitud de onda $\lambda=1310 \mathrm{~nm}$ y polarización TE con el fin de obtener los parámetros de diseño óptimos que minimizasen las pérdidas de acoplo. De manera inicial se optimizó la longitud del taper $(L t)$ y anchura de su parte estrecha $(T w)$ asumiendo un espesor de $1 \mu \mathrm{m}$ para la capa de óxido que cubre el acoplador y de $2 \mu \mathrm{m}$ para la capa de óxido inferior o "Box". En este caso los valores óptimos del taper invertido fueron: $L t=700 \mu \mathrm{m}$ y $T w=70 \mathrm{~nm}$ (figura 3.8(a)). Posteriormente, se hizo una variación de los parámetros de la cubierta de óxido del acoplador, respecto a la anchura y el espesor, siendo óptimos los valores: $W_{\text {cover }}=10 \mu \mathrm{m}$ y $L_{\text {cover }}=1.5 \mu \mathrm{m}$ (figura 3.2), 
alcanzando unas pérdidas de acoplo de 2.4dB (figura 3.8(b)). Finalmente, se hizo una variación con respecto al espesor de la capa de óxido inferior $\left(L_{b o x}\right)$ manteniéndose las pérdidas de acoplo de $2.4 \mathrm{~dB}$ para un espesor de esta capa de $2 \mu \mathrm{m}$ (figura 3.8(c)). Sin embargo, para el desarrollo del láser de cavidad externa se utilizaron obleas de silicio con un espesor a nivel de capa "Box" de $3 \mu \mathrm{m}$, con lo cual las pérdidas de acoplo obtenidas en el diseño del acoplador fueron de $2.6 \mathrm{~dB}$.
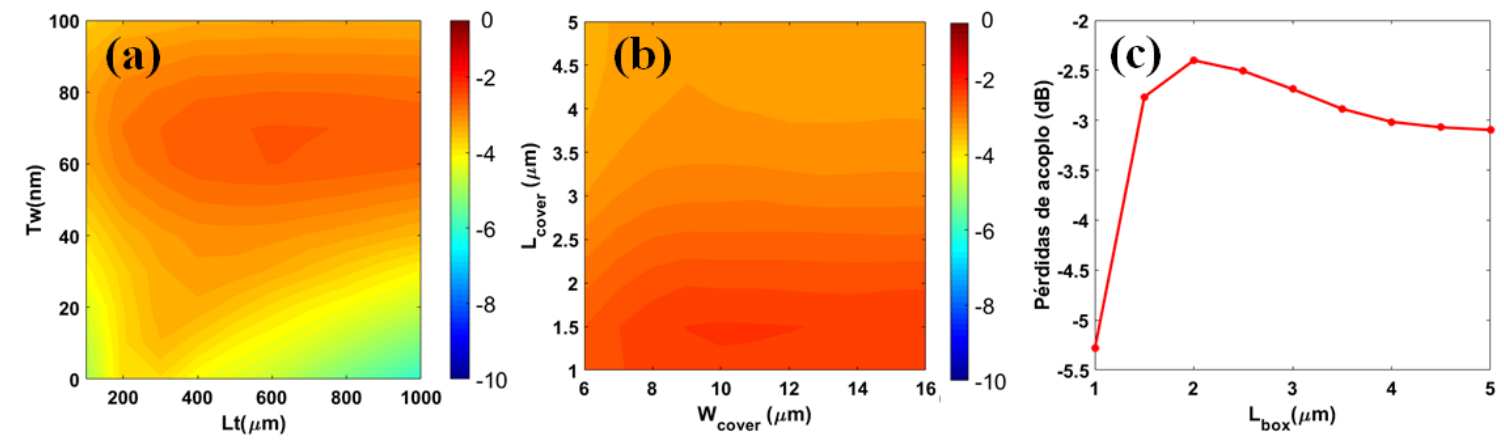

Figura 3.8: Simulaciones 3D-BPM con resolución de 50nm para la obtención de pérdidas de acoplo $(\mathrm{dB})$ en función de: (a) longitud del taper $(L t)$ y el ancho de la punta $(T w)$, (b) espesor y ancho de la cubierta de óxido $\left(L_{c o v e r}, W_{\text {cover }}\right)$, (c) espesor de la capa de óxido $L_{b o x .}$

La longitud del convertidor de tamaño de modo se puede optimizar mejorando el diseño del taper invertido para que el cambio en el factor de confinamiento a lo largo de la estructura sea más rápido. Esto es posible mediante un perfil parabólico formado a partir de uno de los cuadrantes de dos elipses que parten desde la parte ancha del convertidor de tamaño de modo y convergen en su parte estrecha. De esta manera, el ancho del taper que en la estructura inicial cambiaba de manera lineal, ahora cambia de manera parabólica, lo que implica una aceleración en el cambio del factor de confinamiento y como resultado un cambio de tamaño de modo más rápido en una menor longitud de propagación. La estructura y parámetros de diseño se ilustran en la figura 3.9, en la cual se detalla las expresiones geométricas utilizadas en el plano $(x, z)$, correspondiente a la vista superior del acoplador. 


\section{Capítulo 3: Diseño esquema de integración híbrida de láser con cavidad externa}

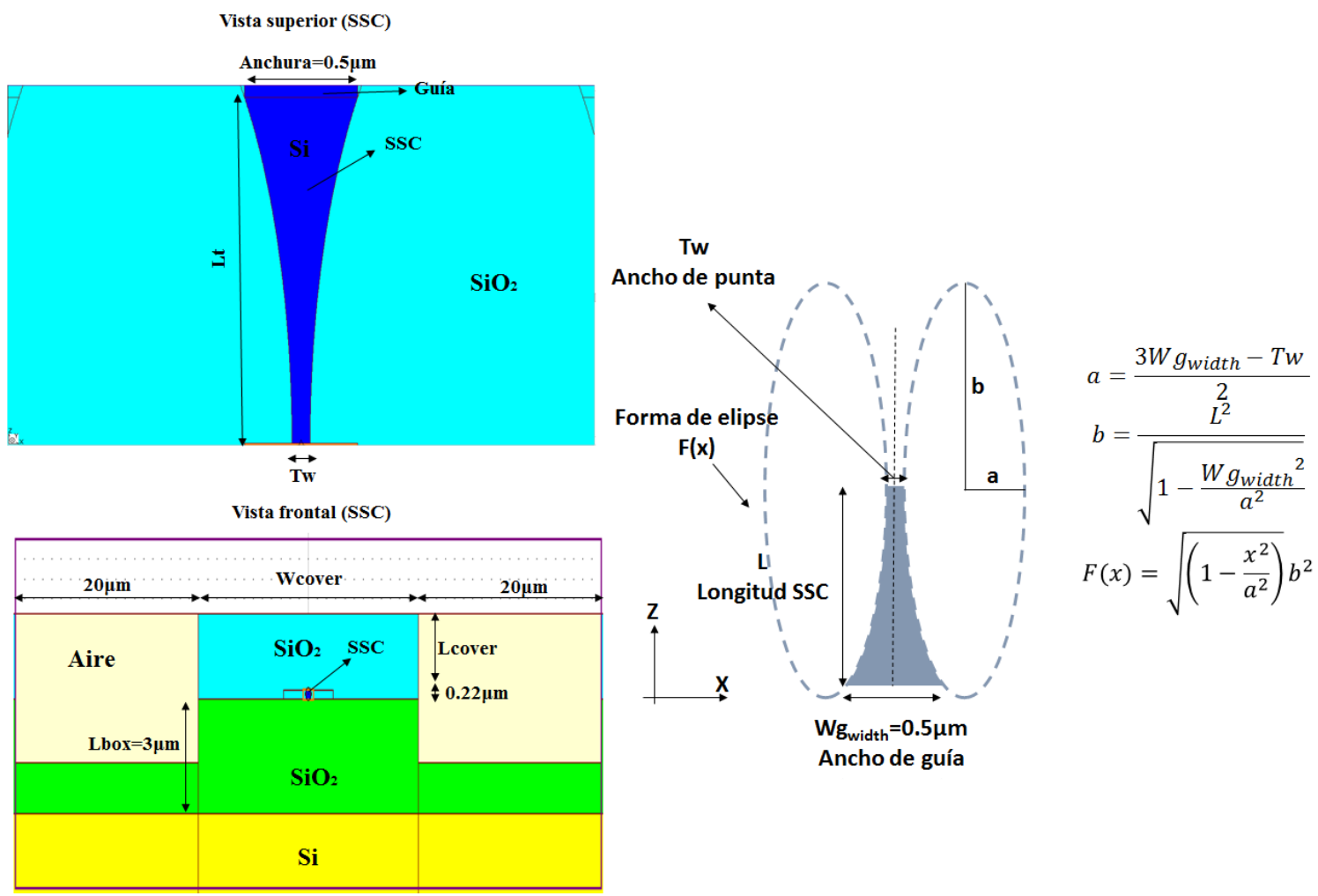

Figura 3.9: Parámetros de diseño para la optimización del convertidor de tamaño de modo por medio de un taper invertido con perfil parabólico. Se detalla la ecuación geométrica de la elipse con los parámetros de diseño del taper invertido, longitud ( $L t)$, ancho de guía $(50 \mathrm{~nm})$ y ancho de punta $(T w)$ en el plano $(\mathrm{x}, \mathrm{z})$.

Para un valor de la capa de óxido inferior $(B o x=3 \mu \mathrm{m})$ y partiendo de los valores de la cubierta superior de óxido tanto a nivel de espesor como anchura ( $L_{\text {cover }}=3.5 \mu \mathrm{m}$, $W_{\text {cover }}=10 \mu \mathrm{m}$ ) se volvió a optimizar la longitud y anchura del taper obteniendo unos valores de $140 \mu \mathrm{m}$ y $80 \mathrm{~nm}$ respectivamente (figura 3.10(a)). Posteriormente, se optimizó el espesor y la anchura de la cubierta de óxido, siendo óptimos $L_{\text {cover }}=2 \mu \mathrm{m}$ y $W_{\text {cover }}=12 \mu \mathrm{m}$ (figura 3.10(b)), alcanzando unas pérdidas de acoplo de $2.5 \mathrm{~dB}$.
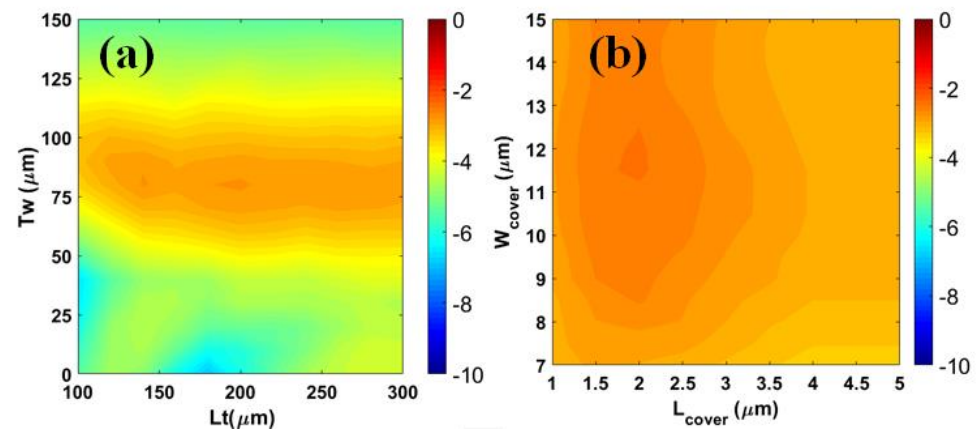

Figura 3.10: Simulaciones 3D-BPM con resolución de 50nm. Pérdidas de acoplo (dB) en función de: (a) longitud del taper $(L t)$ y el ancho de la punta $(T w)$, (b) espesor y ancho de la cubierta de óxido $\left(L_{\text {cover }}, W_{\text {cover }}\right)$. 


\subsection{Diseño del acoplador a láser basado en convertidor de tamaño de modo}

\begin{tabular}{|c|c|}
\hline Parámetros de diseño & Diseño óptimo \\
\hline Longitud del taper & $140 \mu \mathrm{m}$ \\
\hline $\begin{array}{c}\text { Ancho del taper en la } \\
\text { parte estrecha }\end{array}$ & $80 \mathrm{~nm}$ \\
\hline $\begin{array}{c}\text { Ancho de la cubierta } \\
\text { superior de óxido }\end{array}$ & $12 \mu \mathrm{m}$ \\
\hline $\begin{array}{c}\text { Espesor de la cubierta } \\
\text { superior de óxido }\end{array}$ & $2 \mu \mathrm{m}$ \\
\hline
\end{tabular}

Tabla 3.2: Parámetros de diseño óptimos del convertidor de tamaño de modo para el acoplo entre el chip de ganancia QD y una guía SOI de 500nm de ancho, 220nm de espesor y cubierta inferior de óxido de $3 \mu \mathrm{m}$.

Usando los valores óptimos obtenidos en el diseño del acoplador (tabla 3.2), se simuló el modo de propagación excitado por el modo incidente proveniente de una guía (Anchura $=500 \mathrm{~nm}$, Espesor=220nm) en el borde del convertidor de tamaño de modo. A partir de dicho modo, se calculó la eficiencia de acoplo del convertidor de tamaño de modo por medio de la integral de solapamiento

$$
\eta=\frac{\left|\int_{-\infty}^{\infty} \int_{-\infty}^{\infty} E_{1}(x, y) \cdot E_{2}{ }^{*}(x, y) d x d y\right|^{2}}{\int_{-\infty}^{\infty} \int_{-\infty}^{\infty}\left|E_{1}(x, y)\right|^{2} d x d y \cdot \int_{-\infty}^{\infty} \int_{-\infty}^{\infty}\left|E_{2}(x, y)\right|^{2} d x d y}
$$

siendo $E_{1}(x, y)$ el campo del haz de luz incidente proveniente del QD y $E_{2}(x, y)$ el campo del modo de propagación obtenido en el borde del acoplador [87].

Con la integral de solapamiento modelada en Matlab, el modelo de propagación de un haz de luz en espacio libre y el modo de propagación a ser excitado generado por simulación, se comprobaron las pérdidas de acoplo y se estimó la tolerancia respecto a la distancia entre el QD y el SSC, validando el modelo de propagación del haz gaussiano realizado, tal como se detalla en la figura 3.11.
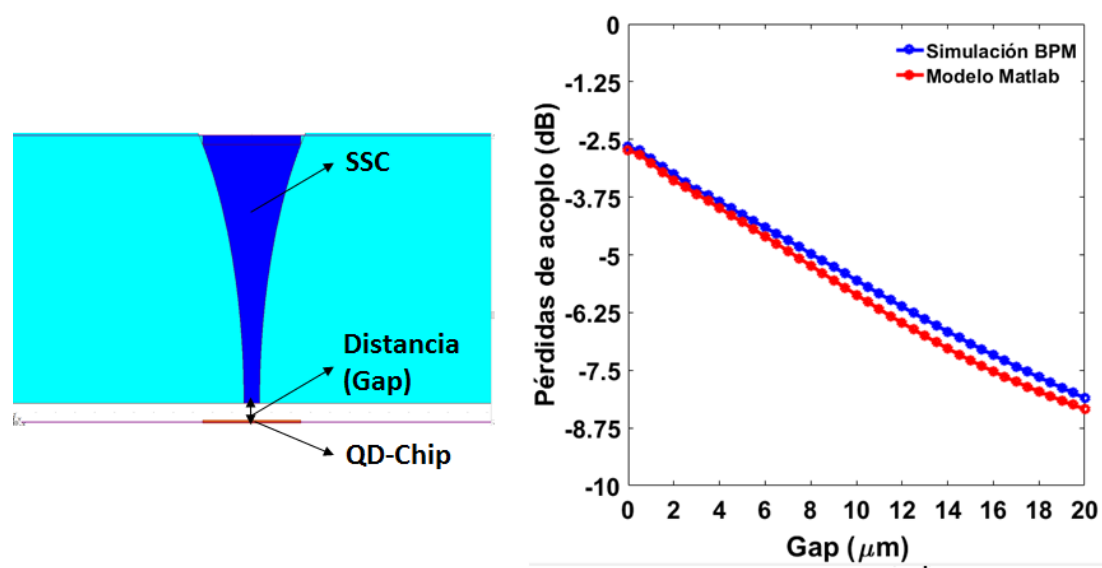

Figura 3.11: Pérdidas de acoplo $(\mathrm{dB})$ en función de la distancia entre el QD y el SSC diseñado, obtenidas con el modelo implementado en Matlab y mediante simulaciones 3D-BPM con resolución de 50nm.

Finalmente, se realizó un análisis de tolerancias respecto a perdidas por desalineamiento mediante simulaciones 3D-BPM, en las cuales se evaluó el desalineamiento del QD respecto a su posición en el plano $(x, y)$. En la figura 3.12(a)) se 


\section{Capítulo 3: Diseño esquema de integración híbrida de láser con cavidad externa}

observa que el desalineamiento en el eje $y$ es más crítico que en el eje $x$. De igual manera, se analizó el desalineamiento respecto al ángulo de emisión del QD en el plano (x,y) (figura 3.12(b)), observándose que un desalineamiento en el ángulo de emisión en el eje $x$ incrementa sustancialmente las pérdidas de acoplo.
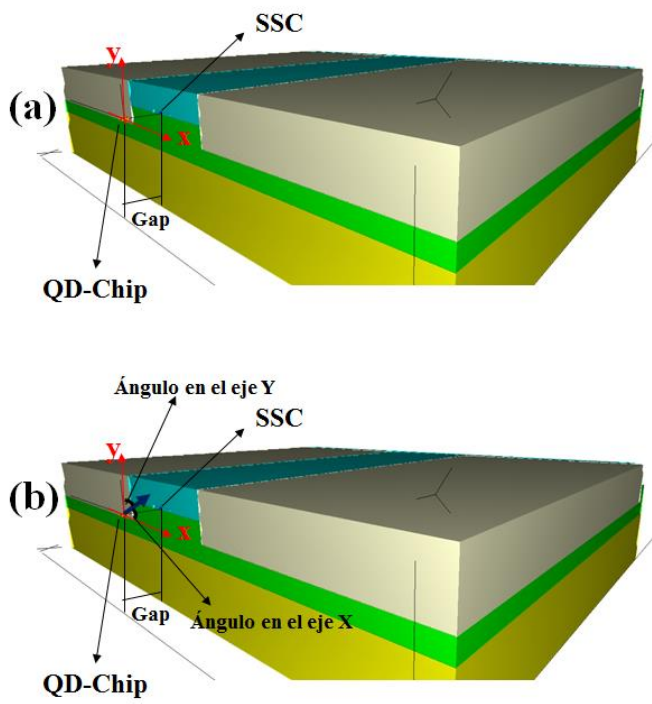
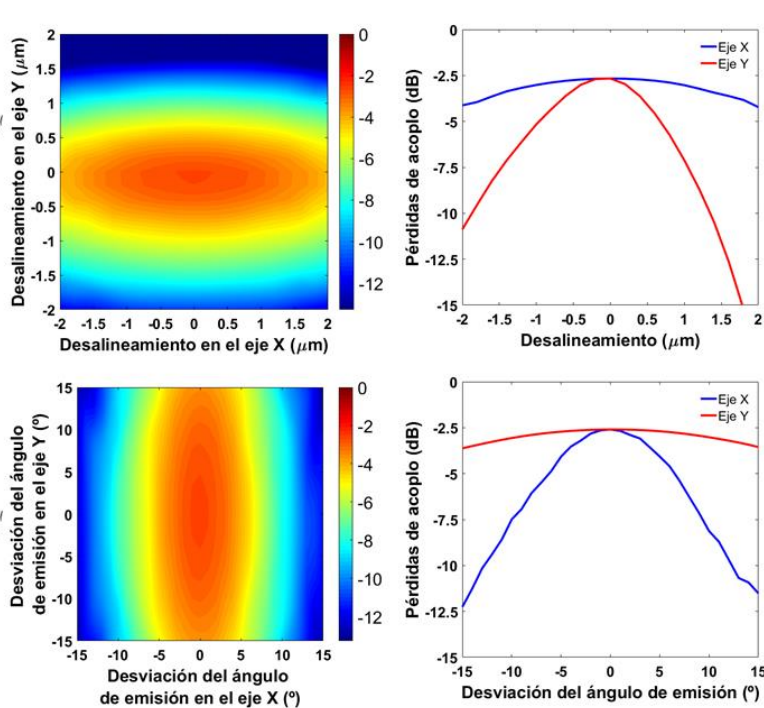

Figura 3.12: Simulación 3D-BPM con resolución 50nm para analizar la tolerancia por desalineamiento debido a (a) desplazamiento del QD y (b) desviación en el ángulo de emisión.

La guía en el QD tiene una inclinación de $5^{\circ}$ respecto a la normal en el punto de emisión para evitar reflexiones, por lo cual se realizó un análisis para determinar el grado de inclinación que debe tener el SSC en el chip de silicio y asegurar un correcto alineamiento. Para dicho análisis se tuvo en cuenta que el medio entre las interfaces del QD y SSC del chip de silicio fuese aire u óxido $\left(\mathrm{SiO}_{2}\right)$, tal como se detalla en la figura 3.13. Para ambos casos, utilizando la ley de Snell, se determinó que el ángulo de inclinación del SSC debía ser de $11.54^{\circ}$.
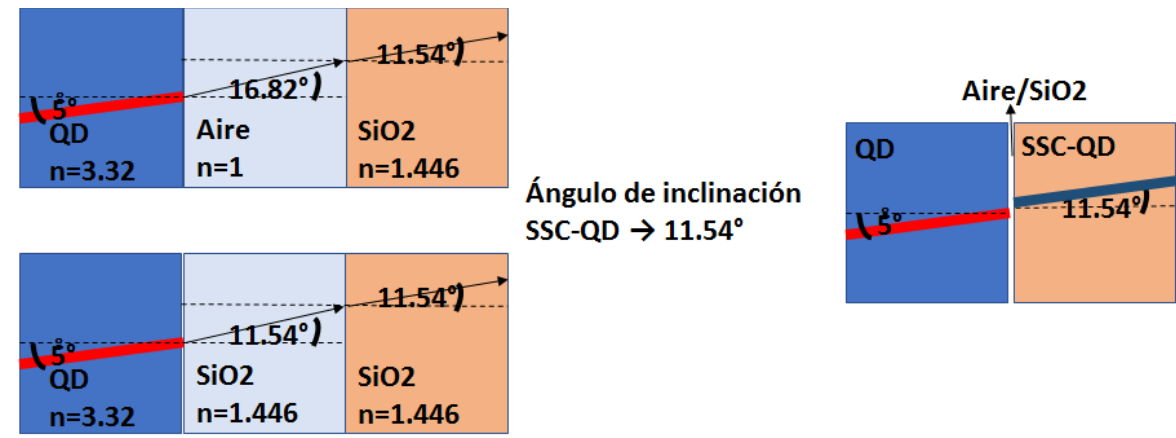

Figura 3.13: Cálculo del ángulo de inclinación del SSC en el chip de silicio para garantizar una correcta alineación con el chip de ganancia QD, cuya guía tiene una inclinación de $5^{\circ}$ respecto a la normal en su interfaz. 


\subsubsection{Análisis de la condición de emisión estimulada}

El análisis de la condición de emisión estimulada requiere conocer las propiedades intrínsecas del QD proporcionadas por el fabricante y detalladas en la tabla 3.3.

\begin{tabular}{|c|c|}
\hline Parámetros & Valor \\
\hline Ganancia saturada $g_{Q D}\left(\frac{N_{p}}{c m}\right)$ & 22 \\
\hline Pérdidas internas $\alpha_{i}\left(\frac{N_{p}}{c m}\right)$ & 2 \\
\hline Eficiencia cuántica interna $\eta_{i}(\%)$ & 69 \\
\hline Índice de refracción material activo $n_{Q D}$ & 3.32 \\
\hline Longitud de la guía de ganancia $L_{Q D}(m m)$ & 1.4 \\
\hline Interfaz altamente reflectante $H R_{Q D}(\%)$ & 99 \\
\hline Recubrimiento anti-reflexiones $A R_{Q D}(\%)$ & 0.01 \\
\hline
\end{tabular}

Tabla 3.3: Parámetros intrínsecos de la fuente de emisión de luz y medio de ganancia QD.

Para que un láser formado por una cavidad externa llegue al punto de emisión estimulada se deben de cumplir dos condiciones básicas: condición de amplitud (Eq. 3.5) y condición de fase (Eq. 3.6). Respecto a la condición de amplitud, para que un láser comience a emitir, las pérdidas dentro de la cavidad, correspondientes a las pérdidas por los reflectores $\alpha_{m}$ y el total de pérdidas en la cavidad externa $\alpha_{E C}$, deben ser menores o iguales a la ganancia provista por el material activo en la cavidad $g_{Q D}$ menos las pérdidas intrínsecas del mismo $\alpha_{i}$ en un recorrido de ida y vuelta. En este sentido, se pueden dar varios casos tal y como se muestra en la figura 3.14. Por un lado, si las pérdidas son mayores y la condición de amplitud no se cumple, se produce una emisión de luz no coherente o espontánea. Por otro lado, si las pérdidas se equiparan al límite de ganancia, el láser comienza a emitir de manera coherente. Finalmente, si las pérdidas están por debajo del límite de ganancia, el nivel de potencia del láser a la salida aumenta. La condición de fase se empleó para un análisis más específico. De esta forma, sólo los modos que cumplan la condición de fase se propagan dentro de la cavidad, y todos los demás son eliminados debido a una interferencia destructiva entre la señal incidente y reflejada en la cavidad. La condición de fase viene impuesta por la suma de la fase del modo propagándose en el medio de ganancia $\phi_{Q D}=\frac{2 \pi n_{Q D}}{\lambda} L_{Q D}$ y la fase del modo propagándose en la cavidad externa $\phi_{\mathrm{EC}}$ en un recorrido de ida y vuelta. Cuando dicha suma da como resultado un múltiplo de $2 \pi q$ siendo $q$ un número entero, el modo se propaga dentro de la cavidad [88].

$$
\begin{gathered}
g_{Q D}-\alpha_{i} \geq \alpha_{m}+\alpha_{E C} \\
2 \phi_{\mathrm{QD}}+2 \phi_{\mathrm{EC}}=2 \pi \mathrm{q}
\end{gathered}
$$


(a)

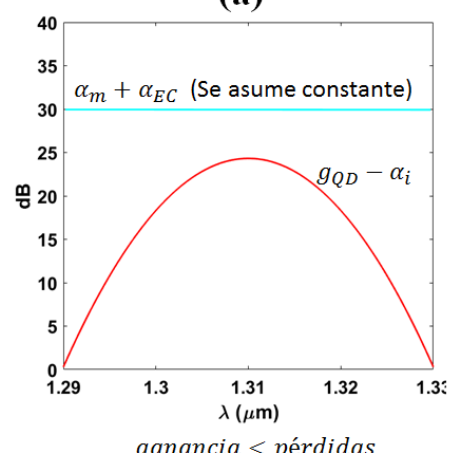

(b)

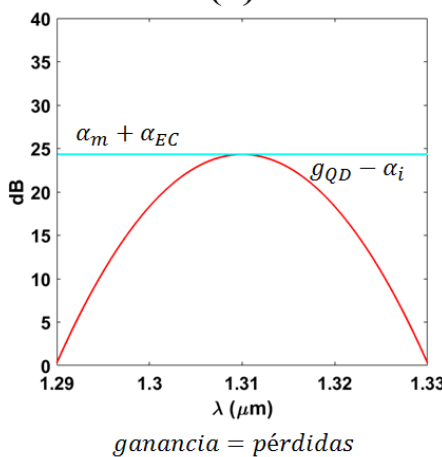

(c)

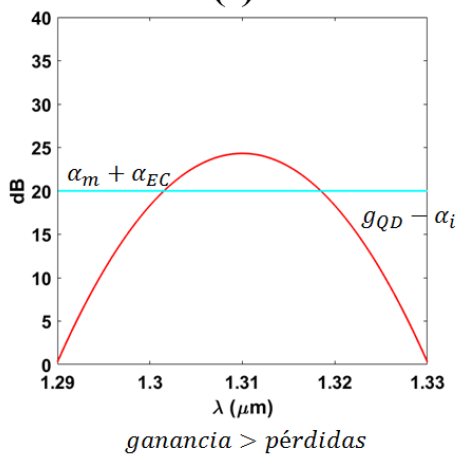

Figura 3.14: Condición de amplitud en la cual si la ganancia es: (a) menor a las pérdidas se producirá una emisión incoherente, (b) igual a las pérdidas, es el límite de ganancia mínimo para producir una emisión estimulada, y (c) mayor a las pérdidas, la potencia de emisión aumenta.

La influencia de cada elemento de la condición de amplitud fue analizada también en detalle. De tal manera, se estimó el funcionamiento del láser de cavidad externa en función de las pérdidas soportadas (figura 3.15). En este sentido, las pérdidas por los reflectores $\alpha_{m}$ son obtenidas por medio de la Eq. 3.7, y el total de pérdidas en la cavidad $\alpha_{E C}$ por la Eq. 3.8, que agrupa las pérdidas en el SSC $\alpha_{S S C}$, las pérdidas de inserción del filtro de anillo resonante $\alpha_{\text {ring }}$ y las pérdidas de propagación en la cavidad externa $\alpha_{\text {prop }} L_{E C}$, todas ellas en un recorrido de ida y vuelta.

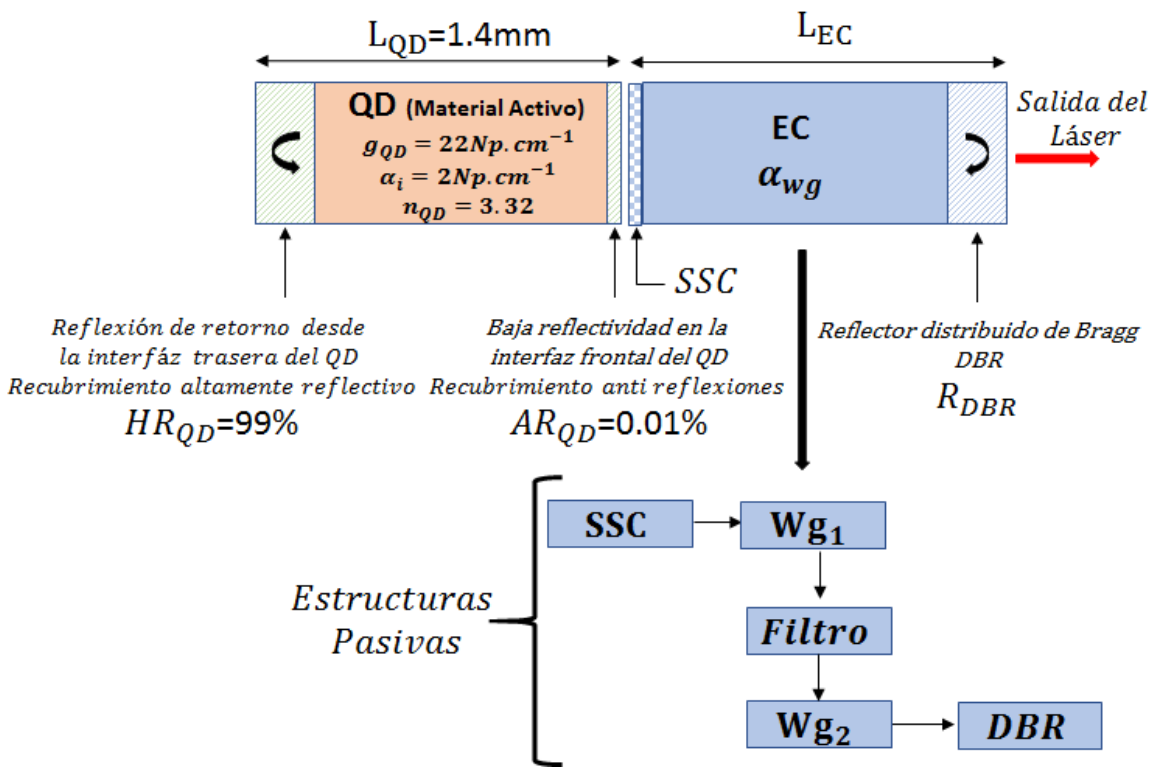

Figura 3.15: Esquema del láser de cavidad externa (ECDL), en el cual se detallan los parámetros del QD y las estructuras de diseño de la cavidad externa.

$$
\begin{gathered}
\alpha_{m}=\frac{1}{L_{Q D}+L_{E C}} \ln \left(\frac{1}{\sqrt{H R_{Q D} R_{D B R}}}\right) \\
\alpha_{E C}=2 \alpha_{S S C}+2 \alpha_{\text {ring }}+\alpha_{\text {prop }} 2 L_{E C}
\end{gathered}
$$


Utilizando la Eq. 3.7, se estimaron de manera inicial las pérdidas en los reflectores en un recorrido de ida y vuelta en función de la reflectividad del reflector distribuido de Bragg o DBR (figura 3.16) como parámetro de diseño de la cavidad externa. En la figura 3.16 se observa que las pérdidas en los reflectores disminuyen a medida que la reflectividad en el DBR aumenta. De tal manera que para una reflectividad del DBR del $50 \%$ y del $95 \%$ las pérdidas en los reflectores son de $1.53 \mathrm{~dB}$ y $0.13 \mathrm{~dB}$ respectivamente.

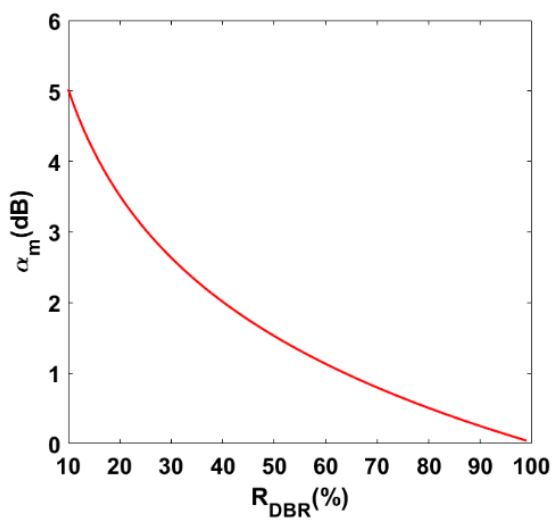

Figura 3.16: Pérdidas en los reflectores de la cavidad en función de la reflectividad del DBR en la cavidad externa.

Para estimar el total de pérdidas en la cavidad externa $\alpha_{E C}$ en un recorrido de ida y vuela se utilizó la Eq. 3.5. Despejando este parámetro se obtuvo el límite de pérdidas en la cavidad externa, que se puede llegar a tener para cumplir la condición de amplitud y obtener una emisión estimulada coherente, en función de la reflectividad del DBR. En la figura 3.17 se observa que el límite de pérdidas totales en la cavidad externa en un recorrido de ida y vuelta se incrementa a medida que la reflectividad aumenta. De esta manera, para una reflectividad del DBR del 50\% y 95\%, el límite de pérdidas totales soportadas en la cavidad externa es de $22.8 \mathrm{~dB}$ y $24.19 \mathrm{~dB}$ respectivamente.

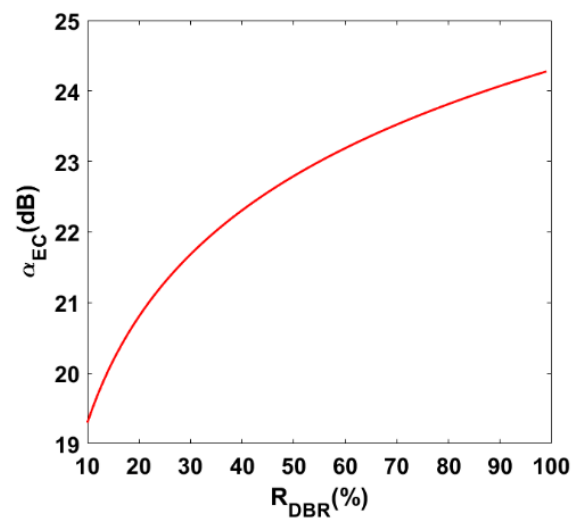

Figura 3.17: Límite de pérdidas soportadas en la cavidad externa en un recorrido de ida y vuelta en función de la reflectividad del DBR para obtener emisión estimulada.

Para estimar las pérdidas de acoplo soportadas en la cavidad externa se utilizó la Eq. 3.8. Partiendo de los resultados anteriormente obtenidos del límite de pérdidas en la 


\section{Capítulo 3: Diseño esquema de integración híbrida de láser con cavidad externa}

cavidad externa (Figura 3.17) y despejando las pérdidas de acoplo, se calculó el límite de pérdidas en el SSC, $\alpha_{S S C}$, en función de la reflectividad del DBR. Para esto se asumieron unas pérdidas de inserción del filtro basado en anillo resonante de $3 \mathrm{~dB}$, además de unas pérdidas de propagación de $30 \mathrm{~dB} / \mathrm{cm}$ (caso más desfavorable). Las pérdidas de propagación en la cavidad externa, $\alpha_{\text {prop }} L_{E C}$, en función de su longitud se detallan en la figura 3.18(a) El límite de pérdidas de acoplo en la cavidad externa estimando una longitud de $370 \mu \mathrm{m}$ se muestra en la figura 3.18(b). Las pérdidas de acoplo soportadas son alrededor de $7 \mathrm{~dB}$ y $8 \mathrm{~dB}$ para una reflectividad del $50 \%$ y $95 \%$ respectivamente.
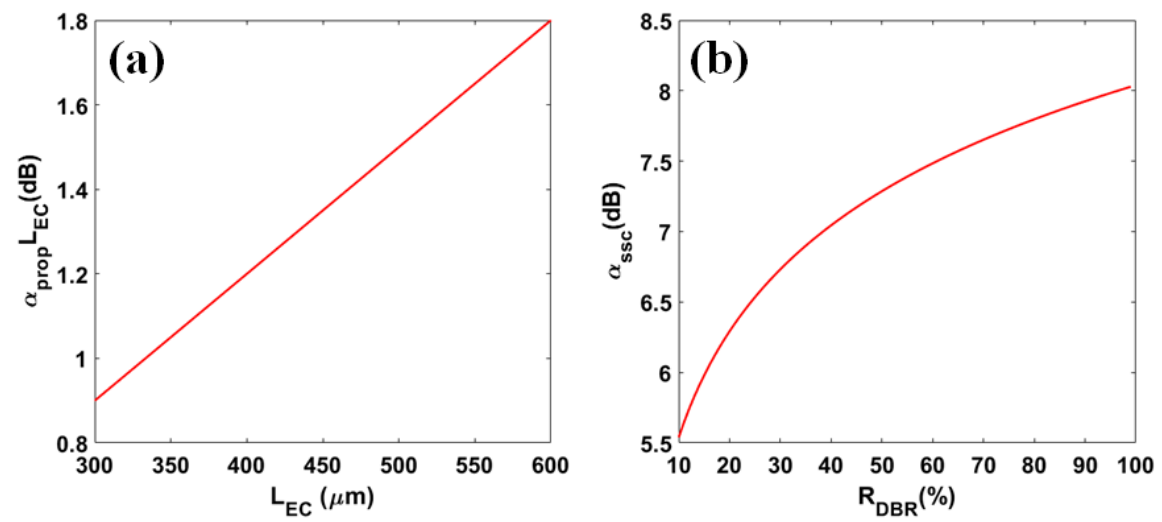

Figura 3.18: (a) Pérdidas de propagación en la cavidad externa en función de la longitud de la cavidad. (b) Límite de pérdidas de acoplo entre el QD y la cavidad externa en función de la reflectividad del DBR para una longitud de $370 \mu \mathrm{m}$.

Tras el cálculo del límite de pérdidas admisibles para que el láser alcance la condición de emisión estimulada coherente, se estimó la potencia de salida del láser mediante la Eq. 3.9. Para ello se calculó la fracción de potencia entregada en el reflector del DBR, $F$ (Eq. 3.10), y el diferencial de eficiencia cuántica $\eta_{d}$ (Eq. 3.11), en función de la reflectividad del DBR, donde $h=6.63 e^{-34}[\mathrm{~J} s]$ representa la constante de Planck, $v=\frac{c}{\lambda}\left[s^{-1}\right]$ representa la frecuencia de oscilación del fotón, $q=1.602 e^{-19}[C]$ representa la carga del electrón, $I_{t h}=0.09[A]$ representa la corriente umbral a la cual el QD pasa de emisión espontánea a estimulada y $I[A]$ representa la corriente que se suministra al QD [89]. Por un lado, en la figura 3.19(a) se observa la fracción de potencia entregada por el DBR, de manera que esta se incrementa a medida que la reflectividad disminuye, y en la figura 3.19(b) se muestra el diferencial de eficiencia cuántica que disminuye a medida que la reflectividad se incrementa. Por otro lado, en la figura 3.19(c) se muestra la potencia de salida del láser obtenida para una reflectividad en el DBR del 50\% y 95\%. Se observa que la potencia aumenta en $20 \mathrm{~dB}$ para ambos casos, medidos a partir de un umbral de corriente de $90 \mathrm{~mA}$ y cuando se alcanza una corriente de $200 \mathrm{~mA}$, correspondiente al valor máximo especificado por él fabricante.

$$
\begin{gathered}
P_{\text {out }}=\eta_{d} \frac{h v}{q}\left(I-I_{T H}\right) \\
\mathrm{F}=\frac{\left(1-\mathrm{R}_{\mathrm{DBR}}\right)}{\left(1-\mathrm{R}_{\mathrm{DBR}}\right)+\sqrt{\frac{\mathrm{R}_{\mathrm{DBR}}}{\mathrm{HR}_{\mathrm{QD}}}}\left(1-\mathrm{HR}_{\mathrm{QD}}\right)}
\end{gathered}
$$




$$
\eta_{d}=F \eta_{i} \frac{\alpha_{m}}{\alpha_{i}+\alpha_{m}}
$$
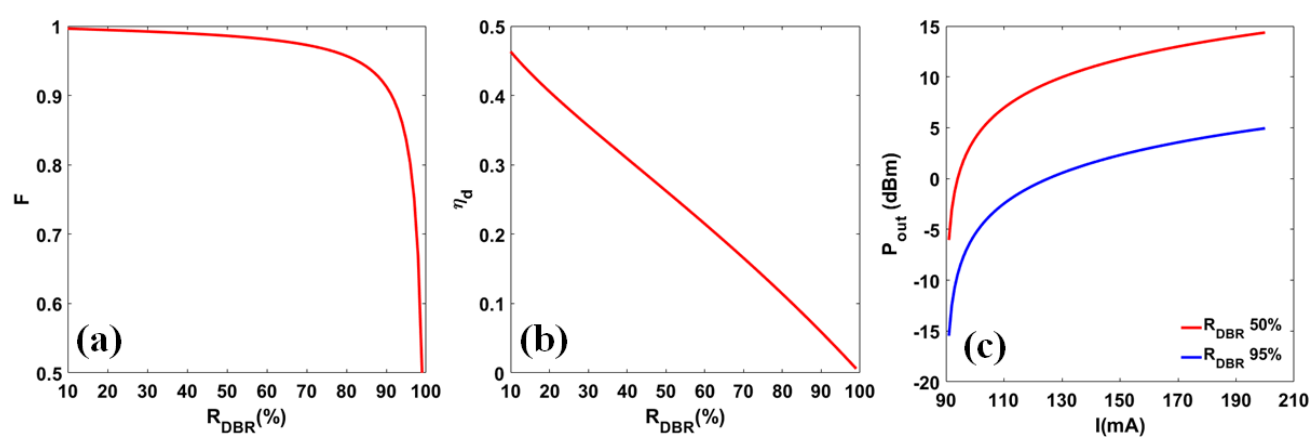

Figura 3.19: Cálculo del factor de ganancia en la potencia de salida del láser obtenido por medio del cálculo de: (a) fracción de potencia entregada por el DBR, (b) diferencial de eficiencia cuántica, (c) potencia de salida del láser para una reflectividad del DBR del 50\% y 95\%.

Después del análisis general de la condición de amplitud se implementó un modelo matemático en Matlab, en el cual se integraron todos los bloques de diseño que conforman la cavidad externa (figura 3.20). De esta manera, se desarrolló un análisis más en detalle sobre el funcionamiento del láser utilizando un modelo de cavidad de tres espejos. Por un lado, en la fuente de emisión de luz QD se dispone de un recubrimiento altamente reflectivo en su parte trasera y en su parte frontal un recubrimiento anti-reflectante. Por otro lado, en la cavidad externa un reflector compuesto por el reflector distribuido de Bragg (DBR) permite el retorno de la luz al medio de ganancia, de tal manera que se forma una cavidad Fabry-Perot. La cavidad externa está compuesta por varias estructuras pasivas que introducen pérdidas: pérdidas de acoplo, pérdidas de propagación en la guía, pérdidas de inserción del filtro basado en anillo resonante y pérdidas en el reflector DBR. El modelo de cavidad de tres espejos permite reducir la cavidad externa en un equivalente de reflectividad $R_{\text {res }}=r_{\text {res }} . r_{\text {res }}^{*}$ y transmisión $T_{\text {res }}=t_{\text {res }} . t_{\text {res }}^{*}$, pudiendo ser calculados por medio de

$$
\begin{array}{r}
r_{\text {res }}=\sqrt{A R_{Q D}}+\frac{P_{D r o p}\left(1-A R_{Q D}\right) \sqrt{R_{D B R}} e^{-2 \gamma_{r e s}}}{1+P_{D r o p} \sqrt{A R_{Q D} R_{D B R}} e^{-2 \gamma r e s}} \\
t_{\text {res }}=\frac{\sqrt{P_{\text {Drop }}\left(1-A R_{Q D}\right)\left(1-R_{D B R}\right)} e^{-\gamma_{\text {res }}}}{1+P_{\text {Drop }} \sqrt{A R_{Q D} R_{D B R}} e^{-2 \gamma r e s}}
\end{array}
$$

siendo * la conjugada de la reflectividad o transmitancia correspondiente a la cavidad formada entre la interfaz frontal del QD con baja reflectividad $A R_{Q D}$ y el reflector de la cavidad externa $R_{D B R}$. Con esta reducción se forma una sola cavidad Fabry-Perot resultante entre la interfaz altamente reflectiva $H R_{Q D}$ del QD y la reflectividad equivalente de la cavidad externa $R_{r e s}$. El coeficiente $\gamma_{r e s}$ viene dado por $\gamma_{r e s}=\alpha_{r e s}-j \phi_{\text {res }}$. Por un lado, la componente de pérdidas $\alpha_{\text {res }}=\alpha_{s s c}+\alpha_{\text {prop }} L_{E C}$, corresponde a las pérdidas de acoplo $\alpha_{s s c}$ y las pérdidas de propagación en la cavidad externa $\alpha_{p r o p} L_{E C}$. Por otro lado, la componente en fase $\phi_{\mathrm{res}}=\frac{2 \pi \mathrm{n}_{\mathrm{eff}}}{\lambda} \mathrm{L}_{\mathrm{EC}}+\phi_{\text {ring }}+\phi_{\mathrm{DBR}}$, corresponde a la fase en la guía 


\section{Capítulo 3: Diseño esquema de integración híbrida de láser con cavidad externa}

de la cavidad externa, $\frac{2 \pi n_{\text {eff }}}{\lambda} L_{E C}$, la fase en el filtro basado en anillo, $\phi_{\text {ring }}$, y la fase en el $\mathrm{DBR}, \phi_{\mathrm{DBR}}$.

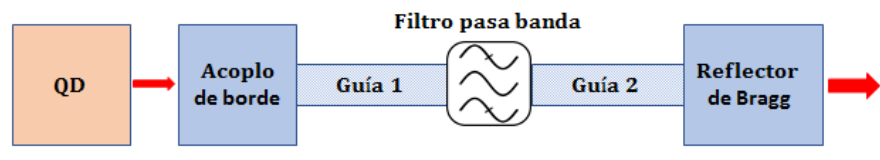

\section{Cavidad Externa}

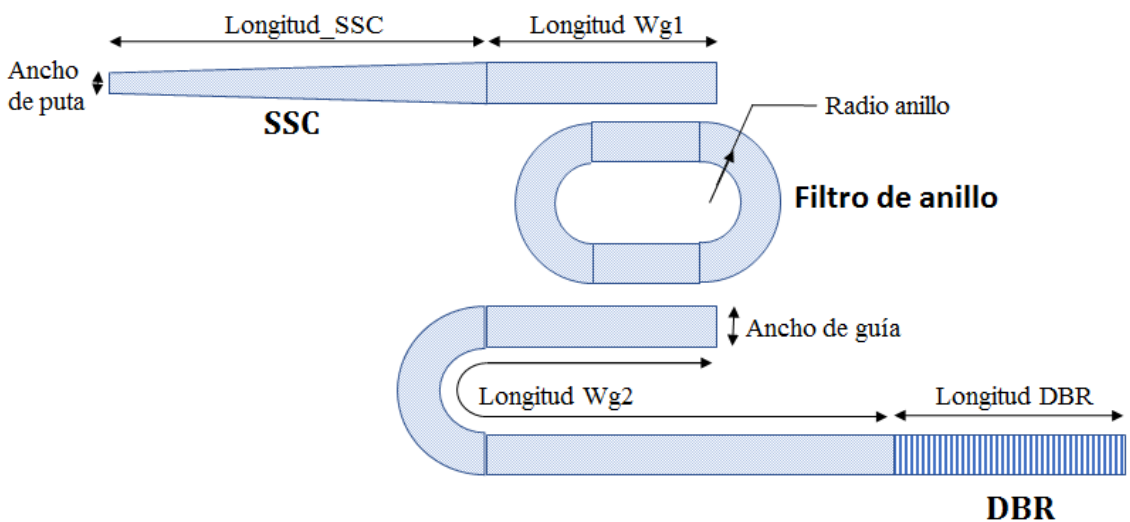

Figura 3.20: Esquema del láser de cavidad externa y bloques de diseño que forman la cavidad externa, acoplador por cambio de tamaño de modo SSC, filtro de anillo y reflector distribuido de Bragg DBR.

En la figura 3.21 se muestra la cavidad resultante a partir del modelo de tres espejos. En este caso, la potencia de salida puede estimarse con

$$
P_{\text {out }}=\frac{\left(1-H R_{Q D}\right) T_{\text {res }} e^{\gamma_{g}}}{\left(1-\sqrt{H_{Q D} R_{r e s}} e^{\gamma_{g}}\right)^{2}+4 \sqrt{H_{Q D} R_{r e s}} \sin \left(\frac{2 \pi n_{Q D}}{\lambda} L_{Q d}+\phi_{\text {res }}\right)^{2} e^{\gamma_{g}}}
$$

donde el coeficiente de ganancia y pérdidas del QD viene dado por $\gamma_{g}=\left(g_{Q D}-\alpha_{i}\right) L_{Q D}$. [90]. Con el modelo implementado y el análisis general realizado, se llevaron a cabo varias simulaciones para analizar el espectro de potencia a la salida del láser.

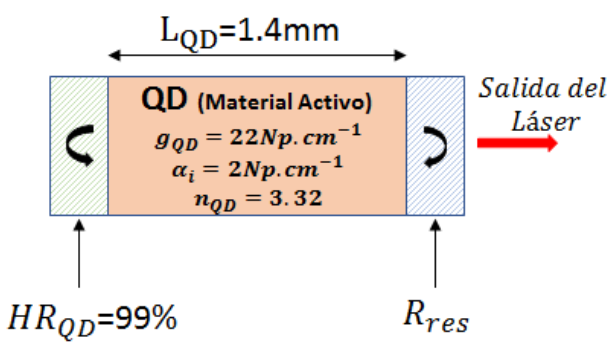

Figura 3.21: Cavidad resultante del modelo de cavidad de tres espejos.

Utilizando el límite de pérdidas de acoplo anteriormente obtenido de $\alpha_{S S C}=7 \mathrm{~dB}$ y $\alpha_{S S C}=8 d B$, y una longitud de cavidad externa de $L_{E C}=370 \mu m$, el espectro de pérdidas en la cavidad $\alpha_{m}$ (Eq. 3.7) se calculó para una reflectividad en el DBR del 50\% y $95 \%$ respectivamente (Figura 3.22(a, d)). Así, en ambos casos las pérdidas en la cavidad son inferiores a la curva de ganancia y las pérdidas intrínsecas en el QD en un recorrido de 
ida y vuelta. Haciendo uso de la Eq.3.6 se calcularon los modos que se propagan en la cavidad, que se detallan en la figura 3.22(b, e), junto a la curva de ganancia y al espectro de pérdidas en la cavidad. Se observa, que sólo un modo de cavidad cumple la condición de amplitud para cada reflectividad. Finalmente, en la figura 3.22(c, f) se muestra el espectro del modo emitido por el dispositivo láser y la posición de los modos de cavidad para cada valor de reflectividad. De esta manera, utilizando el límite de pérdidas de acoplo se comprueba que el láser de cavidad externa es viable para una emisión monomodo.
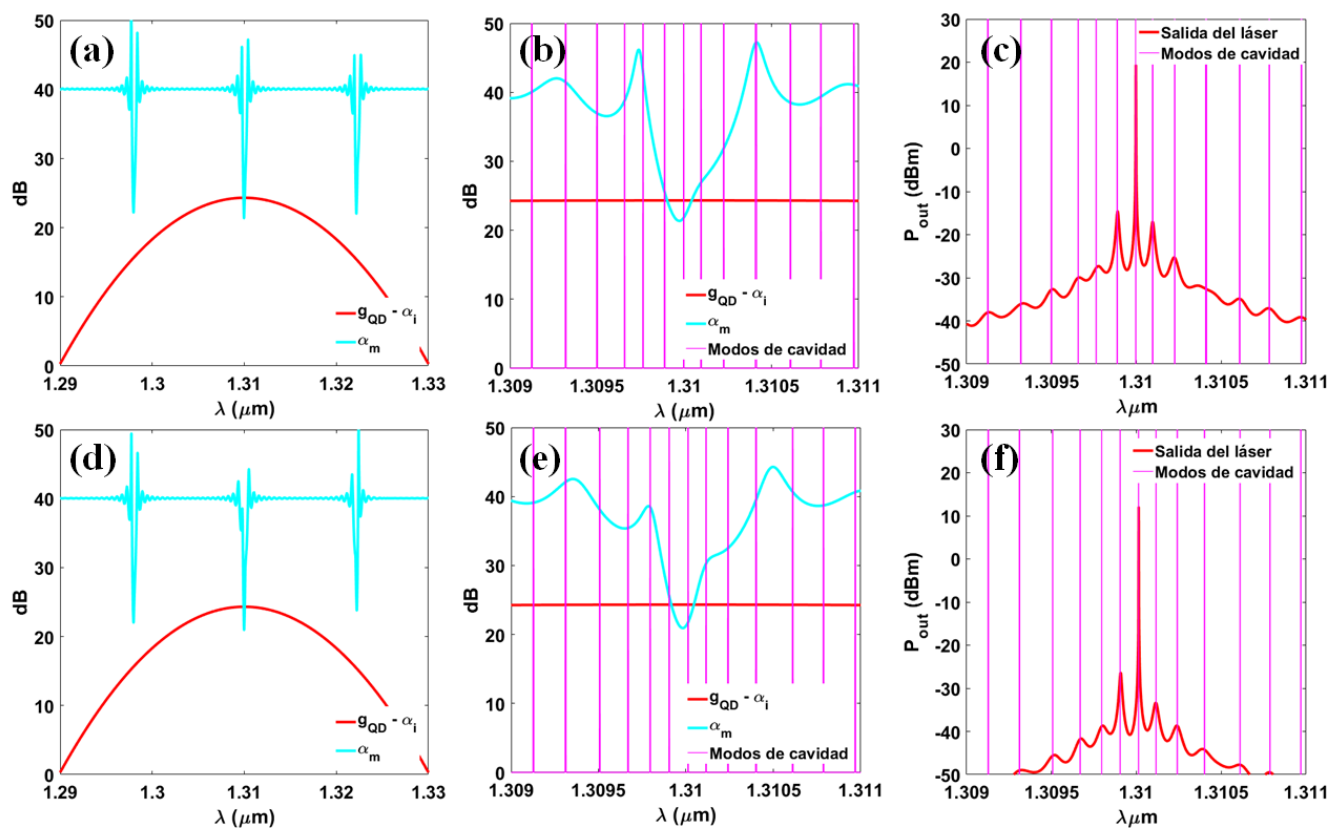

Figura 3.22: Principio de funcionamiento del láser de cavidad externa diseñado: (a, d) pérdidas en la cavidad externa y ganancia en el QD, (b, e) modos que se propagan en la cavidad de acuerdo con la condición de fase, y (c, f) salida del láser de cavidad externa, en todos los casos para reflectividades en el DBR de $50 \%$ y $95 \%$ y límite de pérdidas de acoplo de $7 \mathrm{~dB}$ y $8 \mathrm{~dB}$ respectivamente.

Finalmente, se calculó la separación de los modos longitudinales (Eq. 3.15). De esta manera, para una longitud de guía en la cavidad externa de $L_{E C}=370 \mu \mathrm{m}$ y una longitud del QD de $L_{Q D}=1.4 m m$, siendo $n g_{Q D}$ y $n g_{E C}$ los índices de grupo de las guías del QD y de la cavidad externa, la separación prevista de los modos longitudinales que se propagan en la cavidad es de $F S R_{C M}=0.14 \mathrm{~nm}$, que corresponde a la separación obtenida entre modos de cavidad utilizando la condición de fase (Eq. 3.6).

$$
F S R_{C M}=\frac{\lambda^{2}}{2\left(n g_{Q D} L_{Q D}+n g_{E C} L_{E C}\right)}
$$




\subsection{Diseño del filtro óptico basado en anillo resonante}

El filtro óptico necesario para seleccionar el modo de emisión deseado está basado en un anillo resonante. El anillo resonante actúa como un filtro pasa banda en una configuración conocida como multiplexor óptico de inclusión y descarte u OADM (Optical Add-Drop Multiplexer). Dicha configuración se basa en dos guías rectas que acoplan luz por medio de dos acopladores direccionales a un sólo anillo resonante integrado. De esta manera, ciertas longitudes de onda de la señal óptica provenientes del puerto de entrada son filtradas y guiadas al puerto de salida (Drop) [91]. Para el desarrollo del filtro se utilizó un solo anillo resonante en una configuración "racetrack", tal como se detalla en la figura 3.23 en la cual se incluye los parámetros de diseño de la estructura.

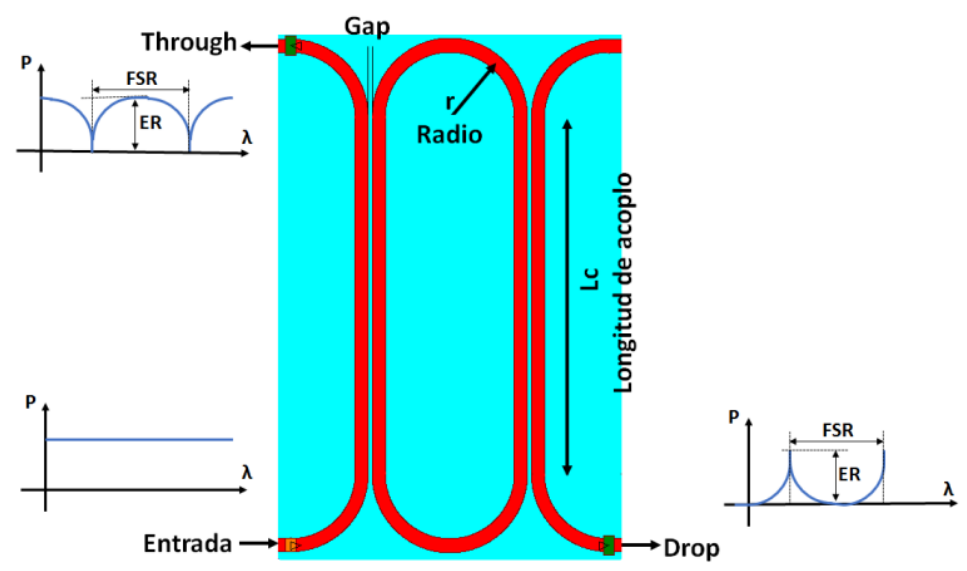

Figura 3.23: Esquema de un filtro OADM en una configuración de anillo "racetrack". Se ilustra el funcionamiento del filtro, dado el espectro óptico de una señal en el puerto de entrada, un filtro rechaza banda será aplicado sobre la señal en el puerto de salida Through y un filtro pasa banda será aplicado sobre la señal en el puerto de salida Drop. El funcionamiento del filtro con una determinada relación de extinción o ER se repite en un rango de longitudes de onda determinado por el (FSR). Los parámetros de diseño son: radio del anillo (r), longitud de acoplo (Lc) y distancia entre las guías y el anillo (gap).

Para alcanzar un solo modo de emisión en el láser en toda la banda, se requiere un valor grande de FSR (Free Spectral Range) en el diseño del anillo. De tal manera, solo una resonancia es deseable dentro del espectro de reflectividad del DBR y el ancho de banda del QD. El diseño de la estructura se realizó para conseguir un valor grande de FSR, bajas pérdidas de inserción en el puerto de salida "Drop" y un nivel alto de relación de extinción de la señal o ER (Extinction Ratio) para maximizar el ratio de supresión de modo lateral o SMSR (Side Mode Suppression Ratio). El diseño y análisis de los parámetros del filtro (figura 3.24) se realizó mediante un modelo en Matlab y simulaciones 3D-FDTD. Por una parte, el radio del anillo es el parámetro más importante para determinar el valor de FSR. Por otra parte, la distancia entre la guía y el anillo, "gap", tiene una gran influencia sobre el factor de acoplo. El valor de FSR es inversamente proporcional a la longitud total del anillo y se puede calcular con la Eq. 3.16, en la cual L es la longitud total del anillo en su configuración "racetrack" (Eq. 3.17), $\lambda$ es la longitud de onda a filtrar y $n_{g}$ es el índice de grupo de la guía del anillo. 

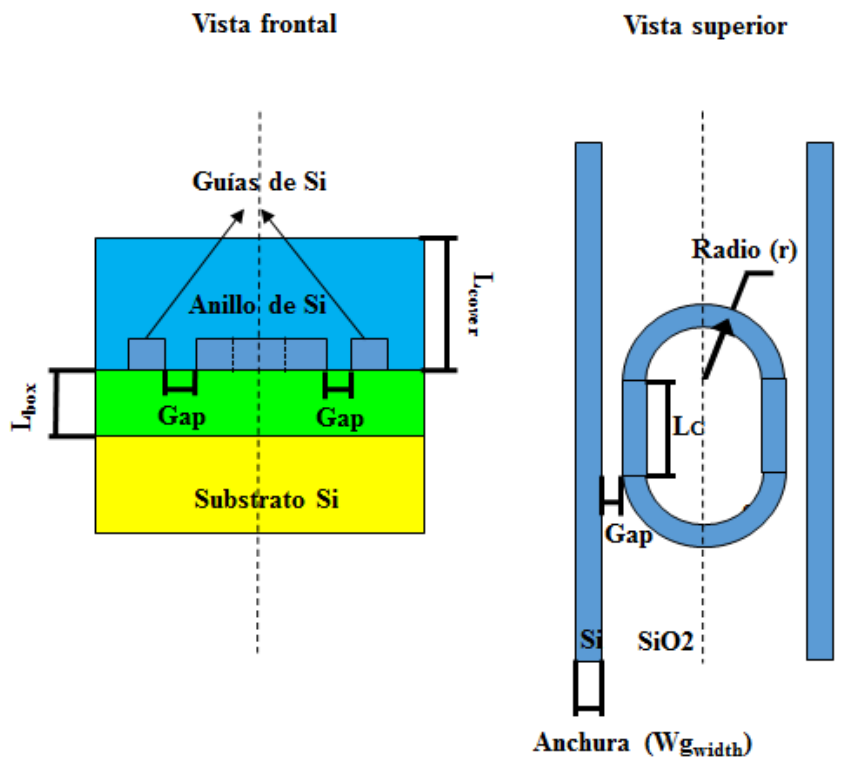

Vista lateral

Figura 3.24: Parámetros de diseño del filtro de anillo resonante. Se detalla las dimensiones del radio del anillo (r), longitud de acoplo $\left(\mathrm{L}_{\mathrm{C}}\right)$, anchura de guía $\left(\mathrm{Wg}_{\text {width }}\right)$, distancia entre la guía y el anillo (gap), espesor de la guía (220nm), y espesor de las capas de óxido ( $\left.\mathrm{L}_{\mathrm{box}}, \mathrm{L}_{\mathrm{cover}}\right)$.

$$
\begin{aligned}
& F S R=\frac{\lambda^{2}}{L n_{g}} \\
& L=2 \pi r+2 L c
\end{aligned}
$$

El objetivo inicial del diseño se enfocó en alcanzar bajas pérdidas de inserción en el puerto "drop" ( $\left.\mathrm{IL}_{\mathrm{drop}}<1 \mathrm{~dB}\right)$ y un alto rango de extinción de la señal $\left(\mathrm{ER}_{\mathrm{drop}}>20 \mathrm{~dB}\right)$. Para lograr dicho objetivo, es necesario un factor de acoplo óptimo. Un valor bajo de acoplo aumenta las pérdidas de inserción, pero mejora la relación de extinción de la señal. Por el contrario, un valor alto de acoplo disminuye las pérdidas de inserción, pero también la relación de extinción de la señal se reduce, tal como se ilustra en la figura 3.25.

$\mathbf{k}<\mathbf{k o p t}$

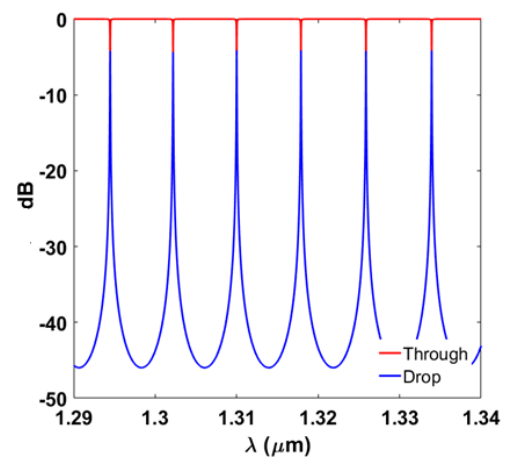

$\mathbf{k} \approx \mathbf{k o p t}$

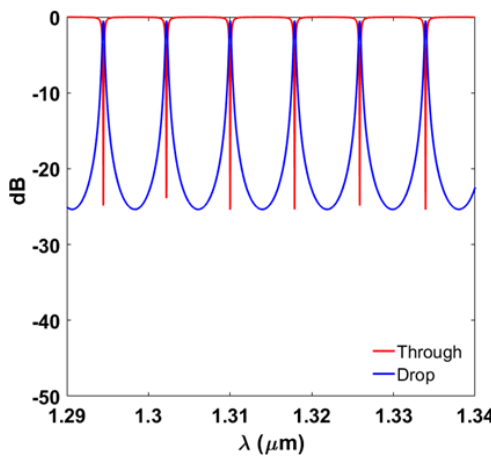

$\mathbf{k}>\mathbf{k o p t}$

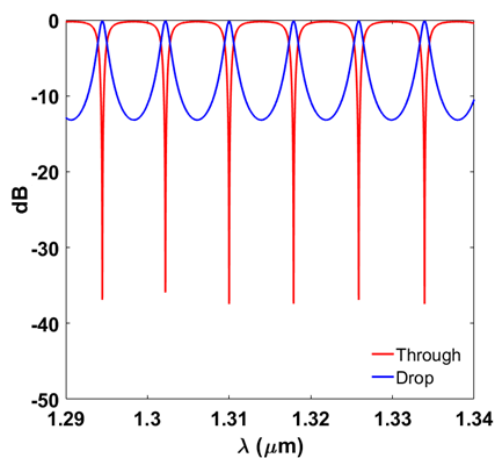

Figura 3.25: Espectro simulado de un filtro OADM en sus puertos de salida "drop" y "through" para diferentes valores de factor de acoplo, $k$.

El funcionamiento del filtro OADM (figura 3.26) puede modelarse mediante ecuaciones [91][92][93] tanto para la salida "through" (Eq. 3.18) como para la salida 


\section{Capítulo 3: Diseño esquema de integración híbrida de láser con cavidad externa}

“drop" (Eq. 3.19), en las cuales $t$ representa el factor de transmisión, $k$ el factor de acoplo y $\gamma=\exp \left(-\alpha \frac{L}{2}+i \beta \frac{L}{2}\right)$, siendo $\alpha$ las pérdidas de propagación, $\beta$ la constante de propagación, $L$ la longitud del anillo y $*$ la conjugada compleja de los valores de $t$ y $k$. Asumiendo una estructura simétrica en la cual los factores de transmisión y acoplo son iguales en ambos acopladores, $t_{1}=t_{2}$ y $k_{1}=k_{2}$, se realizó un estudio para determinar el rango óptimo del factor de acoplo para el cual se cumplen los objetivos iniciales planteados asumiendo unas pérdidas de propagación en la guía del anillo de $10 \mathrm{~dB} / \mathrm{cm}$.

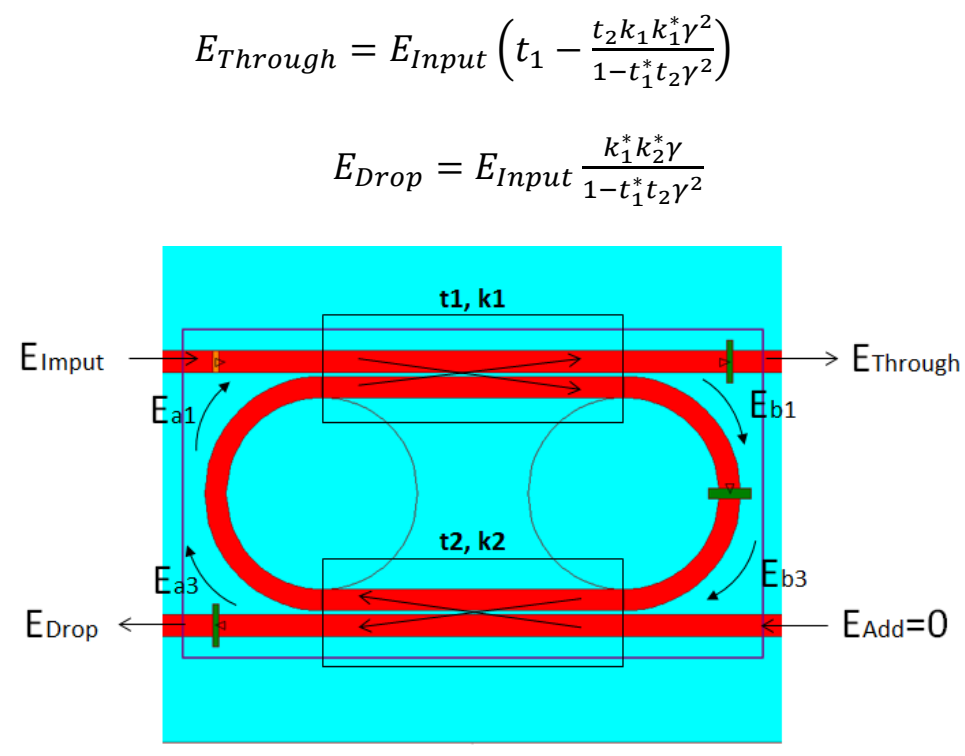

Figura 3.26: Esquema para el modelo matemático de funcionamiento de un filtro OADM.

En la figura 3.27(a) se observa el mínimo valor de factor de acoplo de alrededor de 0.2 con el cual las pérdidas de inserción en el puerto "drop" son menores a $1 \mathrm{~dB}$, mientras que en la figura 3.27(b) se establece el máximo valor de 0.42 para que la relación de extinción de la señal sea mayor o igual a $20 \mathrm{~dB}$. Una mayor relación de extinción de la señal permite alcanzar resonancias más estrechas haciendo la respuesta del filtro más selectiva (figura 3.27(c)). Estos valores se han establecido para diferentes longitudes de anillo con las cuales se puede alcanzar valores de FSR de $8 \mathrm{~nm}$, 10nm y $12 \mathrm{~nm}$.
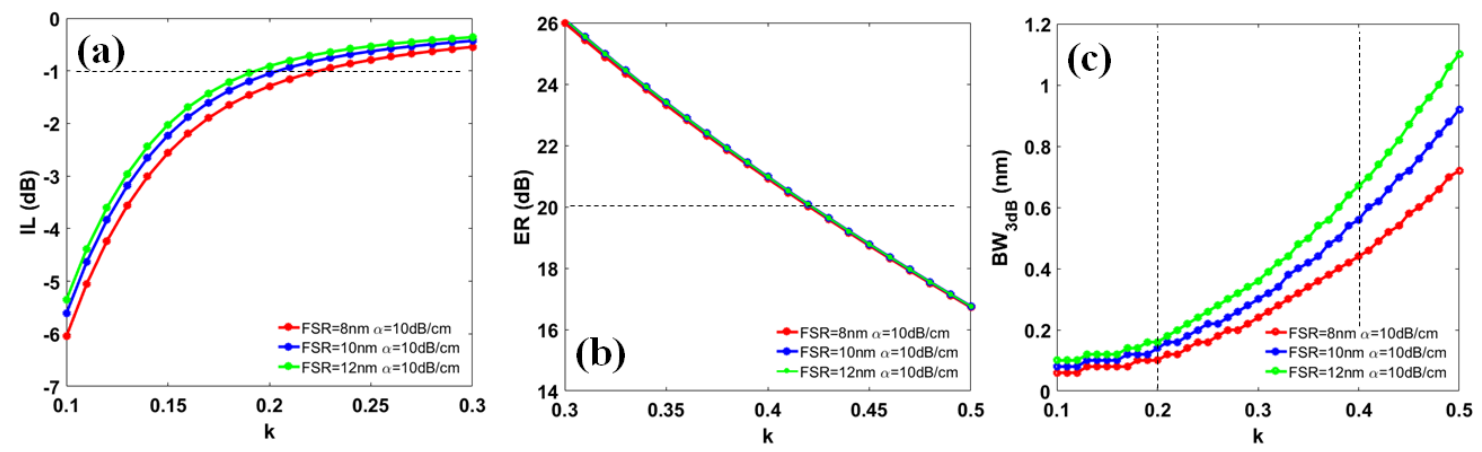

Figura 3.27: (a) Pérdidas de inserción (IL), (b) relación de extinción de la señal (ER) y (c) ancho de banda a $3 \mathrm{~dB}(\mathrm{BW})$ de la respuesta del filtro en el puerto de salida "drop" en función del factor de acoplamiento, $k$, para diferentes valores de FSR. 
Conociendo el rango de valores del factor de acoplo requerido, se realizaron simulaciones 3D-FDTD con resolución de 30nm (figura 3.28(a)) para estimar la longitud de acoplo necesaria. Para ello se consideró una guía SOI de 500nm de ancho, un valor de radio del anillo de $2.5 \mu \mathrm{m}$ y diferentes valores de "gap", de $120 \mathrm{~nm}$ y $140 \mathrm{~nm}$ siendo el primero el mínimo valor posible en fabricación. Como se observa en la figura 3.28(b), el máximo FSR posible para la mínima distancia entre las guías y el anillo ( $g a p=120 \mathrm{~nm}$ ) es de $10 \mathrm{~nm}$ con una longitud de acoplo de alrededor de $L_{c}=12 \mu \mathrm{m}$ para conseguir un factor de acoplo mínimo de $k=0.2$. De esta manera, es necesario incrementar el factor de acoplo sin aumentar la longitud de acoplamiento, ya que, si este valor se incrementa, la longitud total del anillo será mayor reduciendo el valor del FSR. Una alternativa analizada es reducir el ancho de la guía del filtro, de manera que el factor de acoplo se incremente al reducir el factor de confinamiento del modo de propagación en la guía (figura 3.28(c)). Como se observa, al reducir el ancho de la guía el factor de acoplo aumenta, pudiendo alcanzar un FSR mayor con una longitud de acoplo menor y manteniendo la mínima separación entre guía y anillo. En este sentido, con guías de anchura de 460nm y 400nm para un factor de acoplo mínimo correspondiente a longitudes de acoplo $L_{c}=9 \mu \mathrm{m}$ y $L_{c}=4 \mu \mathrm{m}$ se puede alcanzar valores de FSR de alrededor de $12 \mathrm{~nm}$ y $17 \mathrm{~nm}$ respectivamente. Sin embargo, el valor mínimo del factor de acoplo puede verse afectado por desviaciones en el proceso de fabricación. Además, una reducción del ancho de la guía del anillo supone un incremento en las pérdidas de propagación, por lo cual es necesario un análisis de pérdidas y tolerancias.

(a)

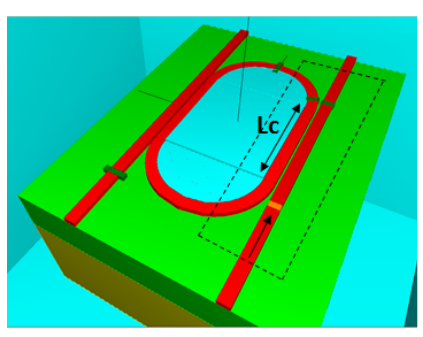

(b)

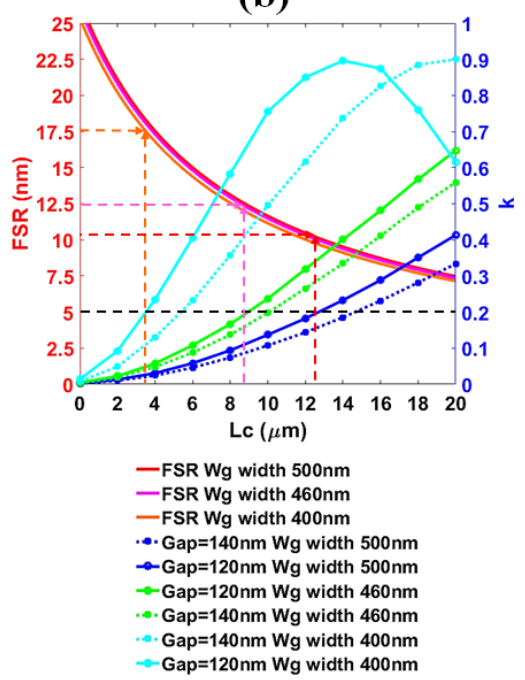

(c)

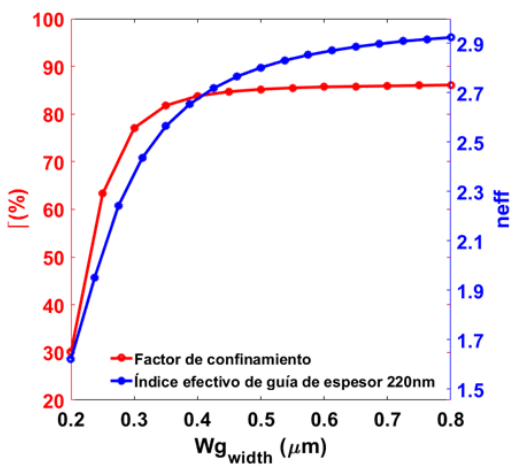

Figura 3.28: (a) Esquema del filtro OADM estructura 3D. (b) Factor de acoplo y FSR (Eq. 3.16) en función de la longitud de acoplo para anillos de radio $2.5 \mu \mathrm{m}$, para diferentes anchos de guía obtenidos a partir de simulaciones 3D-FDTD resolución 30nm. (c) Factor de confinamiento e índice efectivo de propagación en guías de onda de espesor 220nm para diversos anchos de guía, obtenidas a partir de simulaciones 3D-FEM resolución 10nm.

Mediante el modelo en Matlab, se realizó un análisis de pérdidas de propagación en la guía del anillo, teniendo en cuenta varios FSR con un factor de acoplo mínimo en guías de anchura de 500nm, para determinar los efectos sobre las pérdidas de inserción y 


\section{Capítulo 3: Diseño esquema de integración híbrida de láser con cavidad externa}

relación de extinción de la señal en la salida del puerto "drop". En la figura 3.29(a) se observa que el incremento en pérdidas de propagación tiene un mayor impacto sobre las pérdidas de inserción frente a la relación de extinción (figura 3.29(b)). Además, dicho impacto es más acentuado para FSRs altos y factores de acoplo bajos.
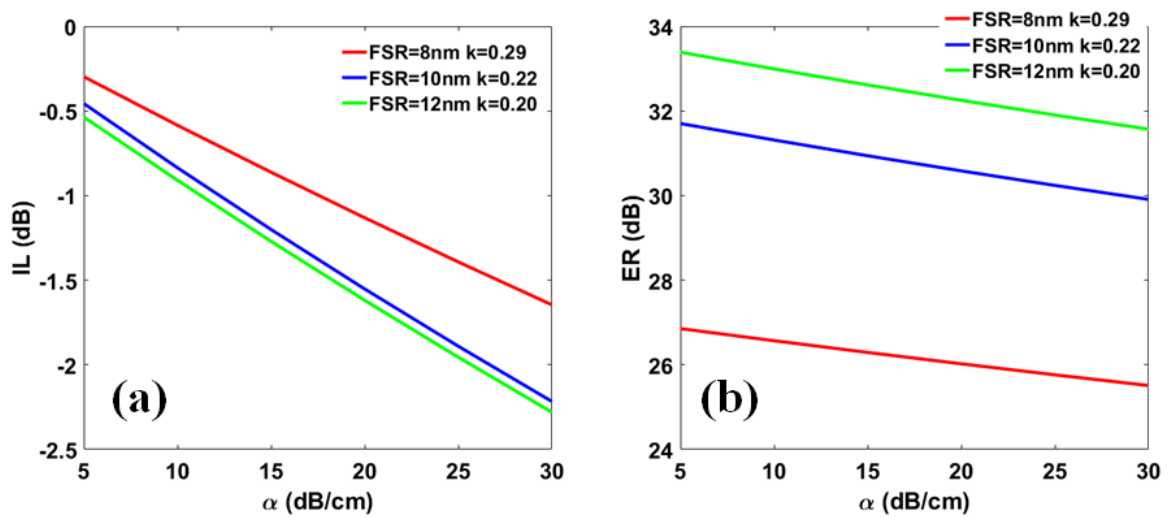

Figura 3.29. (a) Pérdidas de inserción (IL) y (b) rango de extinción de la señal (ER) en función de las pérdidas de propagación, sobre la señal en el puerto "drop" para diferentes valores de FSR.

Por medio de simulaciones 3D-FDTD con resolución de 30nm se realizó un análisis de la tolerancia del filtro frente a variaciones de la separación entre guía y anillo. Para ello se consideraron las separaciones entre guía y anillo de $80 \mathrm{~nm}, 100 \mathrm{~nm}, 120 \mathrm{~nm}$ y $140 \mathrm{~nm}$ y distintos FSRs. La figura 3.30 muestra los resultados obtenidos respecto a la separación entre guía y anillo óptima para cada FSR (Gap=140nm para FSR de 8nm, Gap=120nm para FSR de $10 \mathrm{~nm}$ y Gap=100nm para FSR de $12 \mathrm{~nm}$ ). Se observa claramente como las desviaciones en este parámetro puede tener un fuerte impacto tanto en las pérdidas de inserción como en la relación de extinción, siendo las pérdidas de inserción mayormente afectadas cuando el FSR es mayor. Además, afecta también al ancho de banda de la resonancia tal y como se muestra en la figura 3.30(c). La tolerancia de desviación sobre este parámetro está dentro del rango de $+10 \mathrm{~nm}$ y $-20 \mathrm{~nm}$ para alcanzar unas IL $<1 \mathrm{~dB}$ y un $\mathrm{ER}>20 \mathrm{~dB}$.
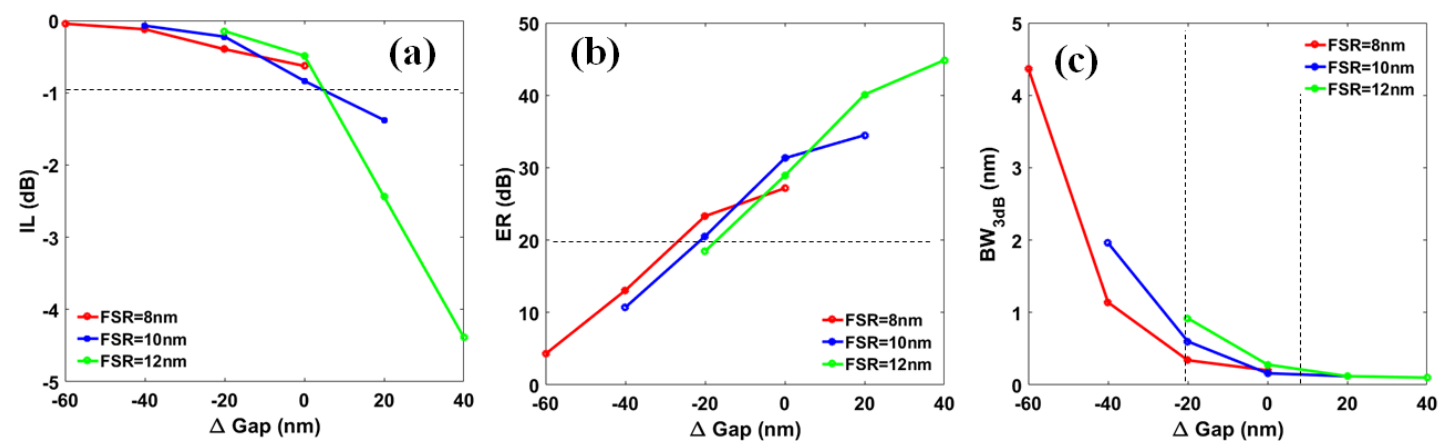

Figura 3.30. Simulaciones 3D-FDTD con resolución 30nm para el análisis de la tolerancia del filtro frente a variaciones de la separación entre guía y anillo. (a) Pérdidas de inserción (IL), (b) relación de extinción de la señal (ER) y (c) ancho de banda (BW) a $3 \mathrm{~dB}$ de la respuesta del filtro en el puerto de salida "drop". 
Finalmente, para la implementación de la cavidad externa, el filtro debe tener un FSR $>10 \mathrm{~nm}$ de tal manera que solo una resonancia del anillo se encuentre en el rango de longitudes de onda deseado (1300nm a 1320nm). De esta manera, se eligió un anillo con un FSR de 12nm, haciendo uso de una guía de 460nm de anchura, siendo sus parámetros de diseño los que se detallan en la tabla 3.4.

\begin{tabular}{|c|c|}
\hline $\begin{array}{l}\text { Parámetros de } \\
\text { diseño }\end{array}$ & Diseño óptimo \\
\hline Ancho de guía & $460 \mathrm{~nm}$ \\
\hline Radio & $2.5 \mu \mathrm{m}$ \\
\hline "Gap" & $120 \mathrm{~nm}$ \\
\hline $\begin{array}{l}\text { Longitud de } \\
\text { acoplo }\end{array}$ & $\begin{array}{l}12.514 \mu \mathrm{m}(\mathrm{FSR}=10 \mathrm{~nm}) \\
9.079 \mu \mathrm{m}(\mathrm{FSR}=12 \mathrm{~nm})\end{array}$ \\
\hline
\end{tabular}

Tabla 3.4: Parámetros de diseño del filtro para alcanzar pérdidas de inserción menores a $1 \mathrm{~dB}$ y una relación de extinción de la señal mayor a 20dB.

El factor de calidad del anillo $Q$ permite conocer la tasa de pérdidas del filtro en relación con la energía acumulada en el anillo y la energía disipada en un ciclo. Un valor alto de este parámetro indica un bajo nivel de pérdidas. Además, permite evaluar la anchura de la resonancia [94]. Este parámetro se obtuvo por medio de la Eq. 3.20 y el cálculo del ancho de banda de la resonancia o FWHM (Eq. 3.21) en el cual $n_{\text {eff }}$ representa el índice efectivo de la guía, $L$ la longitud total del anillo, $\lambda_{\text {res }}$ la longitud de onda de la resonancia y $k$ el factor de acoplo. La anchura de la resonancia calculada para el diseño de FSR $10 \mathrm{~nm}$ y FSR $12 \mathrm{~nm}$ es de $0.58 \mathrm{~nm}$ y $0.27 \mathrm{~nm}$ obteniendo un factor de calidad de alrededor de 2256 y 4915 respectivamente. En la figura 3.31 se muestra el espectro del filtro diseñado de FSR 12nm simulado con Matlab.

$$
\begin{gathered}
Q=\frac{\lambda_{r e s}}{F W H M} \\
F W H M=\frac{k^{2} \lambda_{r e s}^{2}}{\pi L n e f f}
\end{gathered}
$$

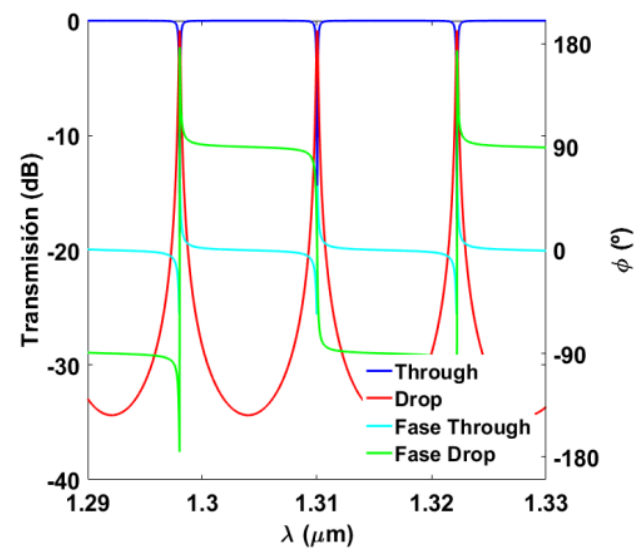

Figura 3.31. Espectro del filtro diseñado basado en un anillo resonante. 


\subsection{Diseño del reflector basado en red de difracción}

El reflector es la estructura fotónica que permite el retorno de la luz en la cavidad externa hacia el chip emisor de luz QD. La estructura considerada está basada en un reflector distribuido de Bragg (DBR). Tanto el valor de la reflectividad como el ancho de banda son parámetros que afectan el funcionamiento del láser, por lo cual se realizó un estudio detallado.

El análisis y posterior diseño del DBR se llevó a cabo mediante un modelo en Matlab siguiendo el flujo de proceso mostrado en la figura 3.32(a). En una etapa inicial se modeló el DBR en el plano bidimensional, sin tener en cuenta el ancho de la guía, haciendo uso de la ecuación de difracción de Bragg para el cálculo de los parámetros de la red de difracción, y de la matriz de transmisión para el cálculo del espectro [95][96][97]. Una vez se desarrolló el modelo, se compararon los resultados de diseño con simulaciones 2DFDTD (figura 3.32(b)), obteniendo los mismos resultados y validando así el modelo. De manera posterior, se tuvo en cuenta la anchura de la guía en el modelo, para un diseño en el plano tridimensional, en el cual el espectro de reflectividad resultante se encuentra centrado a la longitud de onda a la cual la fase es cero. Finalmente, se contrastaron los resultados con simulaciones 3D-FDTD (figura 3.32 (c)).

(a)

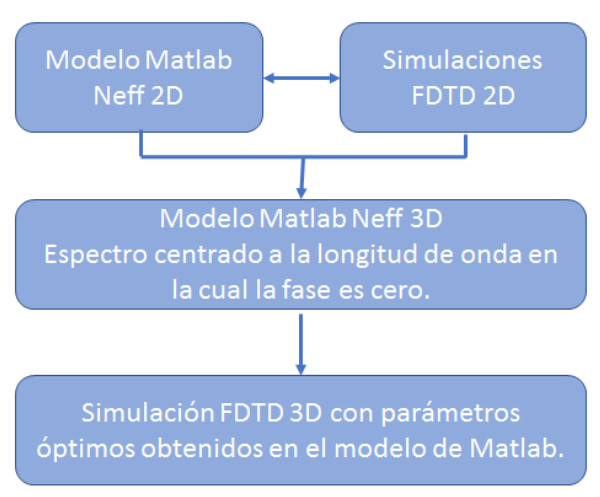

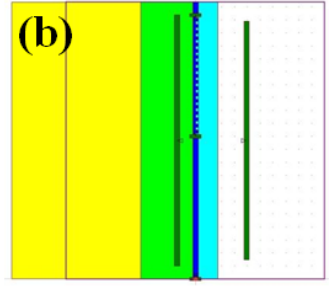

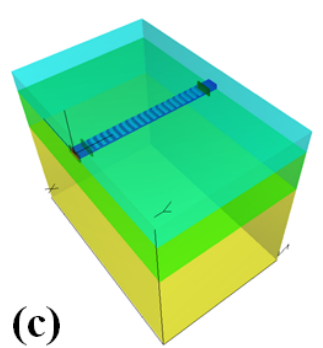

Figura 3.32. (a) Flujo de proceso para el diseño del reflector distribuido de Bragg o DBR. (b) DBR en el plano bidimensional para simulaciones 2D-FDTD. (c) Estructura para simulaciones 3D-FDTD.

Los parámetros de diseño se muestran en la figura 3.33. La optimización del periodo $\Lambda$ y factor de llenado $F f$ se llevó a cabo mediante la ecuación general de difracción (Eq. 3.22), tal y como se detalla en la figura 3.34, en la cual el vector de onda de luz reflejado coincide con el vector de onda de luz incidente proveniente de la guía menos el vector de onda difractado en la red de difracción para un orden de difracción $m=1$. 


\subsection{Diseño del reflector basado en red de difracción}
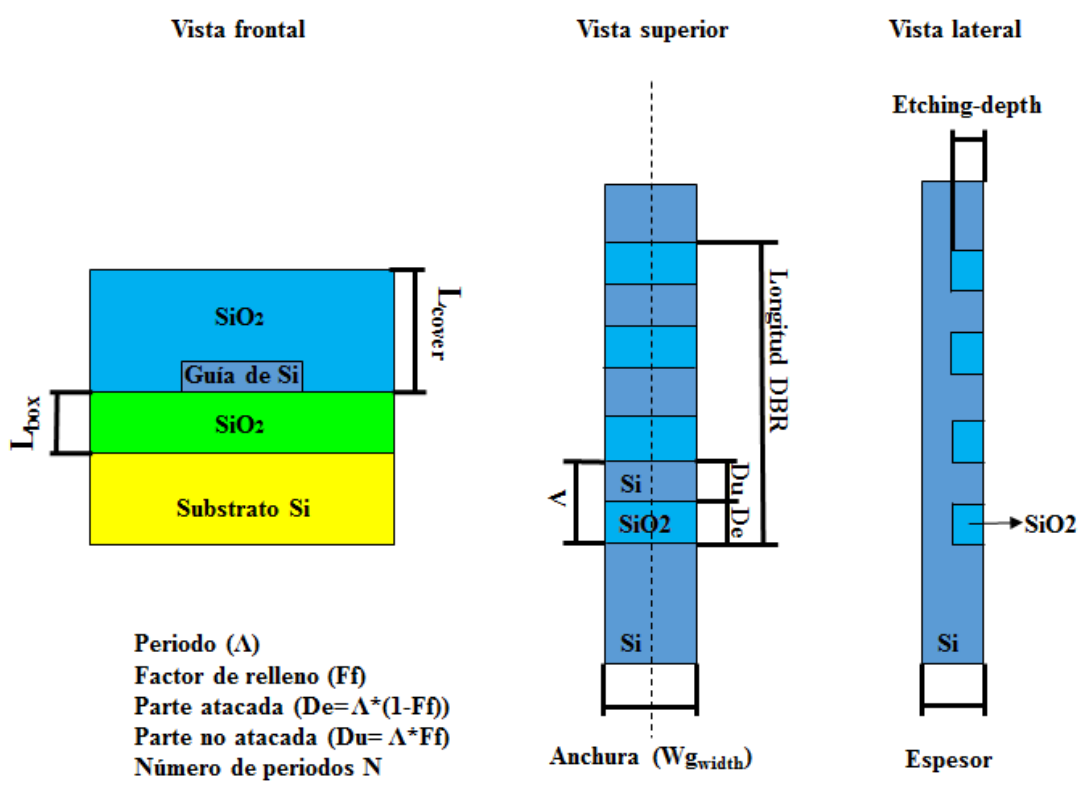

Figura 3.33: Parámetros de diseño del DBR: anchura de guía $\left(W g_{\text {width }}\right)$, periodo de la red de difracción $(\Lambda)$, parte atacada y no atacada $\left(D_{e}, D_{u}\right)$, profundidad de atacado (Etching-depth), espesor de la guía $(220 \mathrm{~nm})$, y espesor de las capas de óxido $\left(L_{c o v e r}, L_{b o x}\right)$.

$$
n e f f_{g} \sin \theta_{m}=n e f f_{w g} \sin \theta_{i n c}-m \frac{\lambda}{\Lambda} \sin \emptyset
$$

El ángulo del vector de onda incidente y resultante respecto a la normal a la superficie de la red de difracción es $\theta_{i n c}=\frac{\pi}{2}$ y $\theta_{m}=\frac{3 \pi}{2}$ respectivamente, mientras que para una interferencia constructiva entre el vector de onda incidente y el vector de onda difractado, los ángulos de ambos vectores respecto a la normal deben sumar $2 \pi$, de manera que $\emptyset=$ $\frac{3 \pi}{2}$, resultando la Eq. 3.23 para el cálculo del periodo de la red de difracción en un DBR.

$$
\Lambda=\frac{\mathrm{m} \lambda}{\text { neff }_{\mathrm{g}}+\text { neff }_{\mathrm{wg}}}
$$

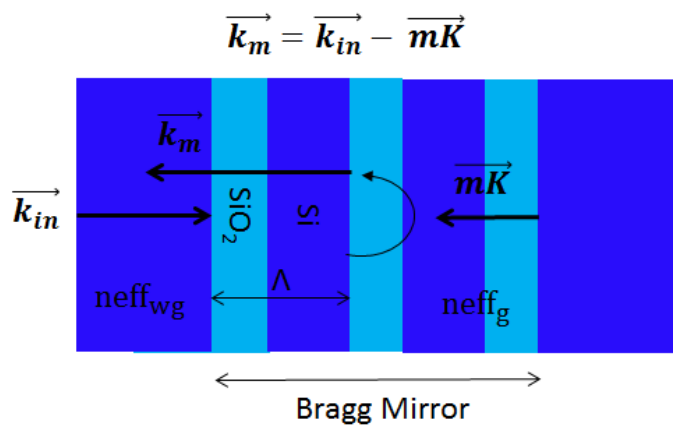

Figura 3.34. Esquema de funcionamiento del reflector basado en una red de difracción (vista superior), en donde $k_{m}$ es el vector de onda resultante, $k_{i n}$ es el vector de onda incidente, $m k$ es el vector de onda difractado, $n e f f_{w g}$ es el índice efectivo de la guía, $n e f f_{g}$ es el índice efectivo de la guía del grating y $\Lambda$ es el periodo de la red. 


\section{Capítulo 3: Diseño esquema de integración híbrida de láser con cavidad externa}

Para el desarrollo del modelo en Matlab basado en matrices de transmisión se tuvieron en cuenta varios parámetros [98][99]. Uno de ellos es el denominado admitancia óptica, $\mu=n e f f Y$, siendo neff el índice efectivo del modo de propagación en la guía y $Y$ (Eq. 3.24) la admitancia o inversa de la impedancia $\left(\eta_{o}=120 \pi\right)$ de una onda en el vacío. Dicho parámetro se calculó en cada una de las partes de la guía de la red de difracción por las cuales se propaga el modo de luz incidente. De este modo, las admitancias en la parte no atacada y atacada de la red de difracción son $\mu_{\text {nep }}=n e f f_{n e p} Y$ y $\mu_{e p}=n e f f_{e p} Y$ respectivamente, tal como se observa en la figura 3.35.

$$
Y=\frac{1}{\eta_{o}}
$$

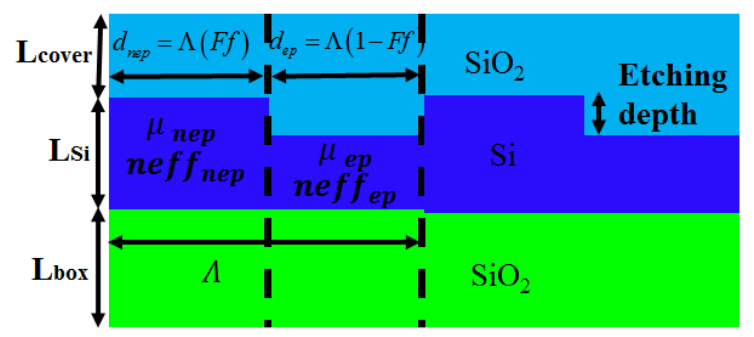

Figura 3.35. Corte longitudinal de la red de difracción para el cálculo de la admitancia óptica en la parte no atacada y atacada de la red. Los parámetros de diseño son: el periodo ( $\Lambda)$, el factor de llenado (Ff), las distancias correspondientes a la parte no atacada y atacada $\left(d_{n e p}\right.$ y $\left.d_{e p}\right)$ y los índices efectivos en cada una de las guías que conforman la red (nef $f_{\text {nep }}$ y nef $f_{e p}$ ).

A continuación, se calculó el cambio de fase $\delta$ en cada una de las partes

$$
\begin{gathered}
\delta_{\text {nep }}=\frac{2 \pi n e f f_{\text {nep }} d_{\text {nep }}}{\lambda} \\
\delta_{e p}=\frac{2 \pi n e f f_{e p} d_{e p}}{\lambda}
\end{gathered}
$$

y con la admitancia y cambio de fase se calculó la matriz de transmisión en cada parte

$$
\begin{aligned}
& M_{\text {nep }}=\left[\begin{array}{cc}
\cos \delta_{\text {nep }} & i \sin \delta_{\text {nep }} / \mu_{\text {nep }} \\
i \mu_{\text {nep }} \sin \delta_{\text {nep }} & \cos \delta_{\text {nep }}
\end{array}\right] \\
& M_{e p}=\left[\begin{array}{cc}
\cos \delta_{e p} & i \sin \delta_{e p} / \mu_{e p} \\
i \mu_{e p} \sin \delta_{e p} & \cos \delta_{e p}
\end{array}\right]
\end{aligned}
$$

Dependiendo del número de capas $(2 \mathrm{n}-1$ en donde $\mathrm{n}$ corresponde al número de periodos de la red), se calculó la matriz de transmisión resultante, si el número de capas es par por medio de la Eq. 3.27 y si es impar con la Eq. 3.28.

$$
M=\left(M_{e p} M_{n e p}\right)^{N / 2}
$$




$$
M=\left(M_{e p} M_{n e p}\right)^{(N-1) / 2} M_{e p}
$$

A partir de los elementos de la matriz resultante definidos como $B=M_{11}+\left(M_{12} \mu_{\text {nep }}\right)$ y $C=M_{21}+\left(M_{22} \mu_{n e p}\right)$, se calculó el espectro de potencia en reflexión $R=r \cdot r^{*}$ y en transmisión $T=t . t^{*}$, siendo ${ }^{*}$ la conjugada de la reflectividad o transmitancia del DBR obtenidos por medio de las ecuaciones Eq. 3.29 y Eq. 3.30 respectivamente.

$$
\begin{gathered}
r=\frac{\mu_{\text {nep }} B-C}{\mu_{\text {nep }} B+C} \\
t=\frac{2 \mu_{n e p} e^{-i n e f f_{\text {nep }}}}{\mu_{n e p} B+C}
\end{gathered}
$$

Haciendo uso del modelo para un atacado de 70nm se calcularon los parámetros de la red de difracción, con los cuales se obtuvieron los espectros de transmisión y reflexión tanto con el modelo implementado en Matlab como por medido de simulaciones 2DFDTD. Como se puede observar en la figura 3.36, los resultados obtenidos en el modelo se ajustan en gran medida a los obtenidos por simulación, validando el funcionamiento del modelo.
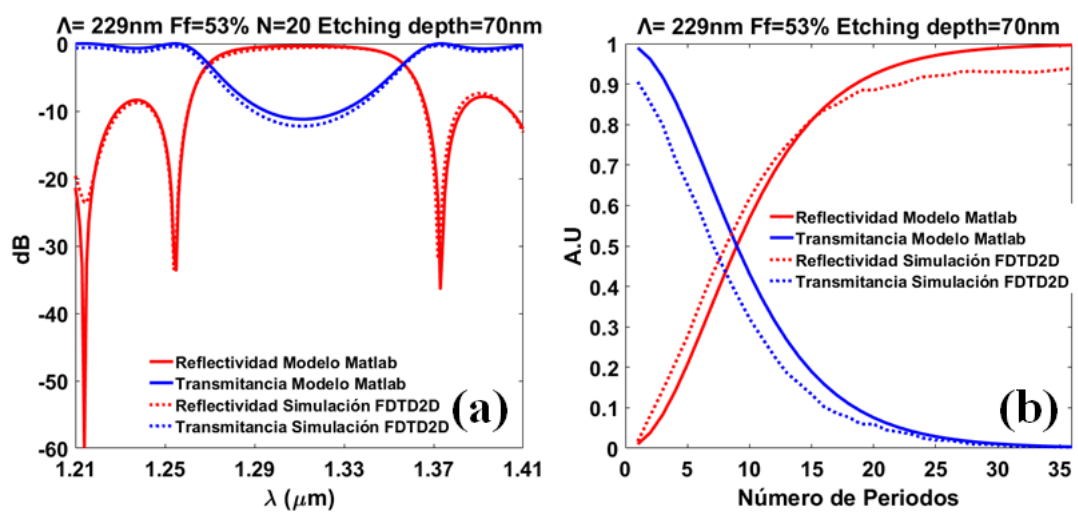

Figura 3.36. (a) Espectro de reflectividad y transmitancia del DBR obtenidos a partir del modelo matemático en Matlab y a través de simulaciones 2D-FDTD con resolución 30nm considerando un número de periodos de $\mathrm{N}=20$. (b) Variación de la reflectividad y transmisión del reflector en función del número de periodos.

Un aspecto que el modelo en Matlab no toma en cuenta es el espesor de las capas de óxido ( $\mathrm{L}_{\text {cover }} \mathrm{y} \mathrm{L}_{\text {box }}$ ) que cubren el reflector, por lo cual se realizaron simulaciones $2 \mathrm{D}$ FDTD para determinar el impacto de la variación de estos parámetros sobre el funcionamiento del DBR. Con los parámetros de diseño anteriormente obtenidos (figura 3.36(a)), en la figura $3.37(a, b)$ se puede observar que la variación de las capas de óxido no tiene gran influencia sobre el funcionamiento del dispositivo tanto a nivel de reflectividad como a nivel de transmitancia. 

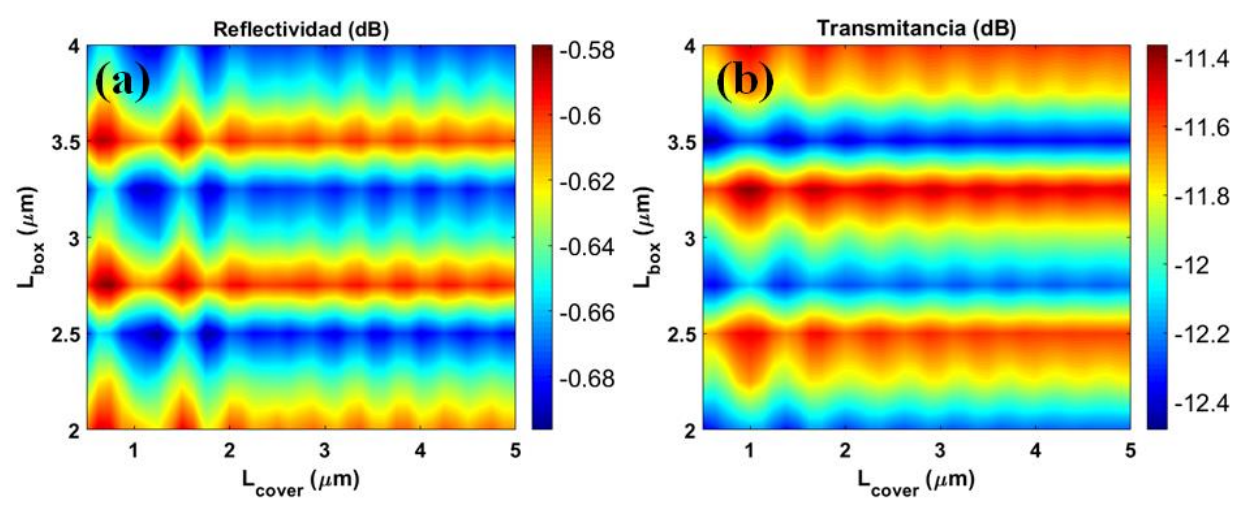

Figura 3.37. Simulaciones 2D-FDTD con resolución 30nm de un DBR con $\Lambda=229 \mathrm{~nm}, F f=0.53$, Etching depth $=70 \mathrm{~nm}$ y $\mathrm{N}=20$ para la obtención de (a) la reflectividad y (b) transmitancia en función de la variación del espesor de las capas de óxido $L_{c o v e r}$ y $L_{b o x}$.

El modelo del reflector se diseñó en el plano bidimensional asumiendo una anchura de guía infinito y por tanto unas dimensiones de anchura de la guía mucho mayor que la longitud de onda. Sin embargo, para dimensiones de anchura de guía inferiores a la longitud de onda, no se puede asumir una anchura de guía infinita por lo cual un modelo que contemple este parámetro es necesario. Para incorporar la anchura de la guía en el modelo, se calcularon los índices efectivos de las guías que componen la red de difracción sobre una estructura 3D haciendo uso del método del índice efectivo. Utilizando los índices efectivos de las guías para una anchura de 500nm, se obtuvieron reflectores con diversas profundidades de atacado, tal como se muestra en la tabla 3.5. El funcionamiento de cada diseño se analizó inicialmente con el modelo implementado en Matlab (figura 3.38(a)), para el cual se puede observar que para un mismo número de periodos a mayor profundidad de atacado mayor es la reflectividad. Para validar los resultados del modelo, se realizaron simulaciones 3D-FDTD (figura 3.38(b)) observándose una menor reflectividad que la obtenida con el modelo teórico, debido a pérdidas de luz difractada al substrato y aire que no pueden ser tenidas en cuenta en el modelo. Sin embargo, el ancho del espectro tiene una gran similitud.

\begin{tabular}{|c|c|c|c|}
\hline $\mathbf{A}(\mathbf{n m})$ & Ff $(\%)$ & Etching depth $(\mathbf{n m})$ & $\mathbf{N}$ \\
\hline 242 & 50 & 10 & 20 \\
\hline 246 & 48.98 & 30 & 20 \\
\hline 250 & 48.58 & 50 & 20 \\
\hline 256 & 47.74 & 70 & 20 \\
\hline 264 & 46.64 & 90 & 20 \\
\hline 275 & 45.34 & 110 & 20 \\
\hline 289 & 43.68 & 130 & 20 \\
\hline
\end{tabular}

Tabla 3.5: Parámetros del DBR diseñado para una guía de 500nm de ancho considerando diversas profundidades de ataque. 
(a)
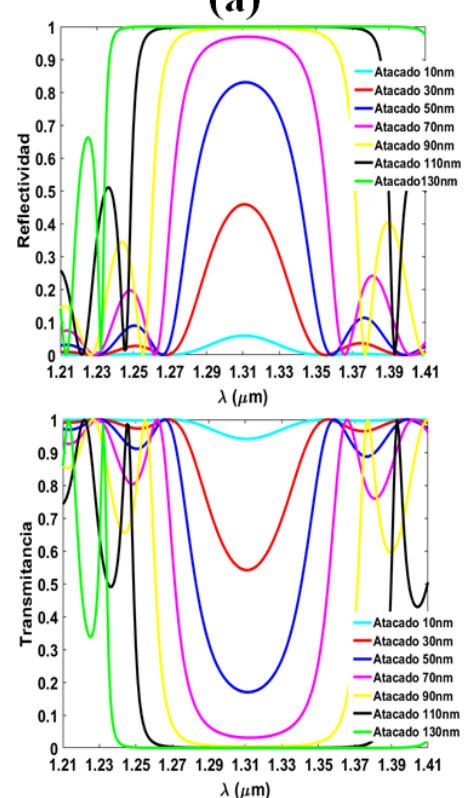

(b)

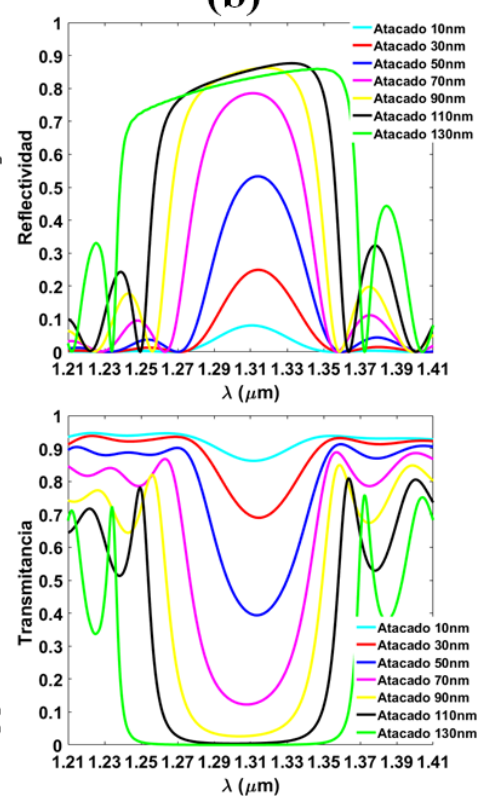

Figura 3.38. (a) Modelado Matlab y (b) simulaciones FDTD 3D resolución 30nm, del funcionamiento de un DBR diseñando sobre una guía de anchura 500nm, para obtener el espectro de reflectividad y transmitancia para diversas profundidades de ataque.

En el diseño del láser, el ancho de banda del espectro de reflectividad del DBR debe ser lo suficientemente ancho para cubrir el rango de longitudes de onda de interés, $1300 \mathrm{~nm}-1320 \mathrm{~nm}$, pero a su vez debe ser lo suficientemente estrecho para que solo una resonancia del filtro de anillo este dentro del ancho de banda y así lograr un funcionamiento monomodo. El ancho de banda del reflector disminuye a medida que la profundidad de ataque es menor, tal y como se observa en la figura 3.38. Sin embargo, profundidades de ataque por debajo de los 30nm están limitadas tecnológicamente debido al proceso de fabricación, por lo cual la profundidad de atacado se fijó en 30nm. Para este valor se calculó el periodo y factor de llenado, de tal manera que el espectro de reflectividad del DBR debía estar centrado a una longitud de onda de 1310nm y el número de periodos debía ser ajustado para obtener la reflectividad/transmitancia deseada y un ancho de banda de alrededor de 20nm. De esta manera, los parámetros óptimos obtenidos para el diseño del DBR fueron de $246 \mathrm{~nm}$ de periodo y del $49 \%$ de factor de llenado, centrado a una longitud de onda de $1310 \mathrm{~nm}$ a la cual la fase del espectro de reflectividad es igual a cero (figura 3.39(a)). El valor de la reflectividad y ancho de banda depende del número de periodos, de tal manera que a mayor número de periodos la reflectividad será mayor y el ancho de banda más estrecho (figura 3.39 (b, c)). Por contra, un bajo número de periodo, y por lo tanto una baja reflectividad, hace que la respuesta del espectro de reflectividad se ensanche, incrementando el ancho de banda (figura 3.39 (b, d)). 

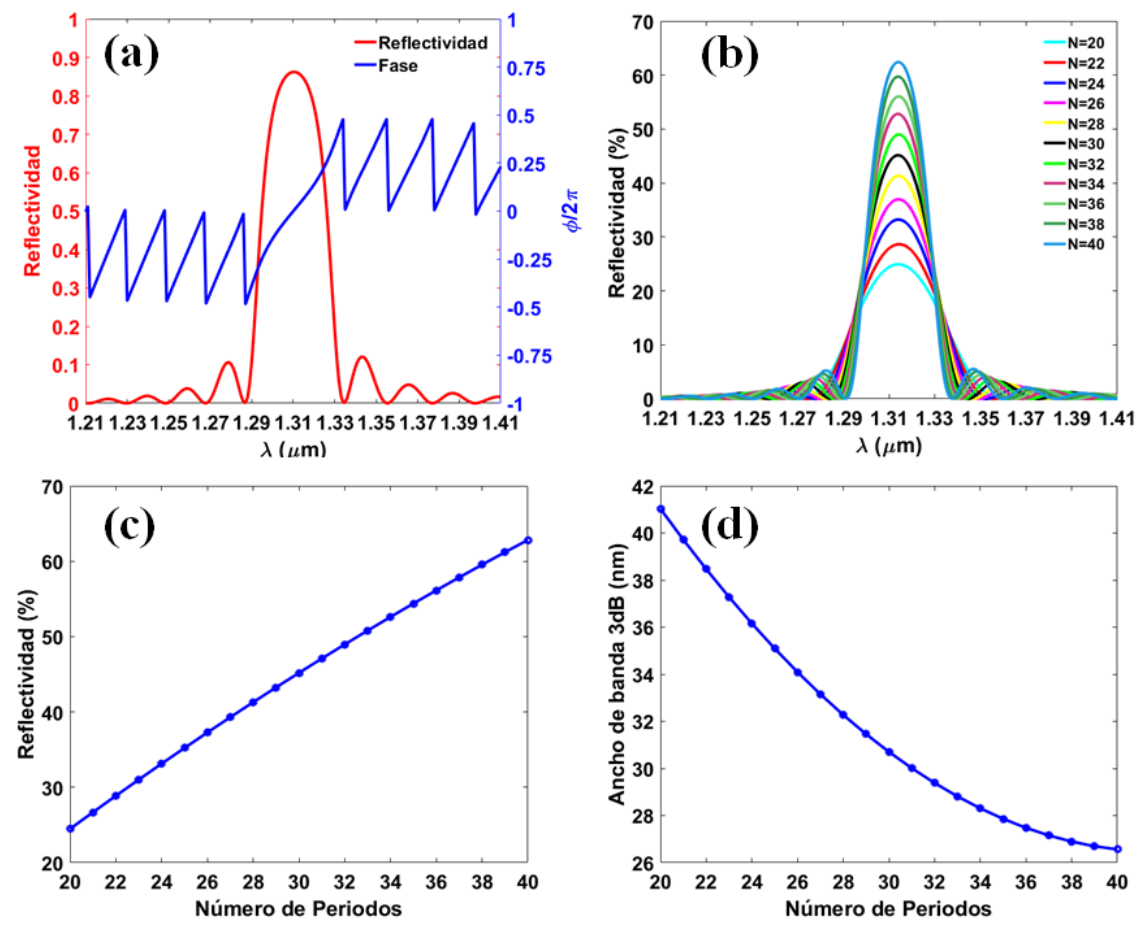

Figura 3.39. (a) Espectro de reflectividad y fase del DBR obtenidos con el modelo de Matlab para un periodo de $246 \mathrm{~nm}$ y un factor de llenado del 49\%. Simulaciones 3D-FDTD con resolución 30nm del (b) espectro de reflectividad para un diferente número de periodos, (c) reflectividad en función del número de periodos y (d) ancho de banda a $3 \mathrm{~dB}$ en función del número de periodos.

Los parámetros de diseño del DBR de acuerdo con los valores de reflectividad deseables para la implementación del láser se muestran en la tabla 3.6.

\begin{tabular}{|c|c|c|c|c|c|}
\hline $\begin{array}{c}\text { Parámetros } \\
\text { de diseño }\end{array}$ & Diseño Óptimo & Reflectividad & $\begin{array}{c}\text { Ancho de Banda } \\
3 \mathrm{~dB}\end{array}$ & $\begin{array}{c}\text { Número de periodos }=60 \\
\text { Reflectividad }\end{array}$ & $\begin{array}{c}\text { Ancho de Banda } \\
3 \mathrm{~dB}\end{array}$ \\
\hline $\begin{array}{c}\text { Periodo } \\
\begin{array}{c}\text { Factor de } \\
\text { llenado }\end{array}\end{array}$ & $246 \mathrm{~nm}$ & & & & \\
\hline $\begin{array}{c}\text { Profundidad } \\
\text { de atacado }\end{array}$ & $39 \%$ & $50 \%$ & $30 \mathrm{~nm}$ & $95 \%$ & $20 \mathrm{~nm}$ \\
\hline
\end{tabular}

Tabla 3.6: Parámetros de diseño óptimos y características del espectro (reflectividad y ancho de banda) obtenidos con el modelo de Matlab y simulaciones 3D-FDTD.

A partir del diseño óptimo, se realizó un análisis de tolerancias frente a posibles desviaciones en fabricación por medio de simulaciones 3D-FDTD. Por un lado, una variación en la profundidad de atacado produce un ligero desplazamiento del espectro del DBR respecto a la longitud de onda de diseño, sin embargo, tiene un alto impacto en la reflectividad. Si la profundidad de atacado es mayor a la del diseño se produce un desplazamiento hacia longitudes de onda menores y se incrementa el valor de la reflectividad, tal y como se observa en la figura 3.40(a). Concretamente, una variación de la profundidad de atacado de $\pm 10 \mathrm{~nm}$ provoca un desplazamiento del espectro de $\pm 6 \mathrm{~nm}$ respecto a la longitud de onda de diseño y un $\pm 16 \%$ de variación a nivel de reflectividad. Por otro lado, una variación del factor de llenado desplaza ligeramente el espectro del DBR respecto a la longitud de onda de diseño y reduce la reflectividad estimada. Si el 
factor de llenado es mayor que el de diseño se produce un desplazamiento hacia longitudes de onda mayores, mientras que si es menor el desplazamiento se da hacia longitudes de onda menores. En ambos casos la reflectividad se reduce levemente tal y como se muestra en la figura 3.40(b). Concretamente, una variación del factor de llenado de $\pm 10 \%$ provoca un desplazamiento de $\pm 3 \mathrm{~nm}$ y una reducción en reflectividad de $2.5 \%$. De esta manera, se observa que el DBR es más robusto frente a desviaciones del factor de llenado. Finalmente, se analizó la variación del periodo, obteniéndose un gran impacto respecto al desplazamiento del espectro del DBR y una menor incidencia sobre la reflectividad. Un periodo mayor al de diseño produce un desplazamiento hacia longitudes de onda mayores y un incremento en reflectividad despreciable, mientras que si es menor el desplazamiento se da hacia longitudes de onda menores acompañado de una reducción también despreciable en reflectividad, tal como se muestra en la figura 3.40(c). De esta forma, una variación en el periodo de $\pm 5 \mathrm{~nm}$ provoca un desplazamiento del espectro de $\pm 18 \mathrm{~nm}$.
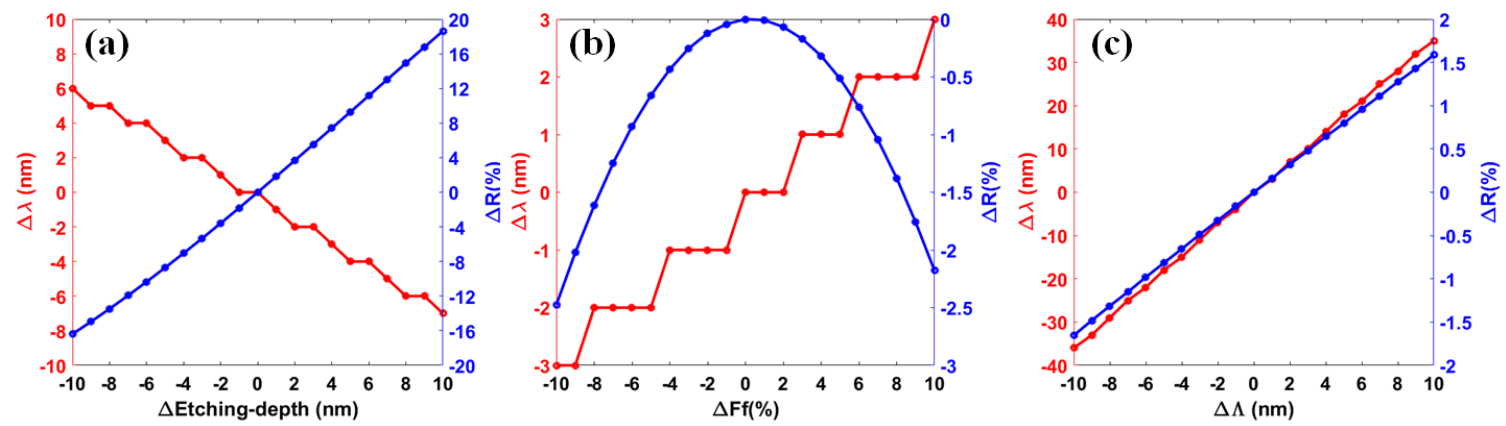

Figura 3.40. Desplazamiento del espectro de reflectividad del DBR y variación de la reflectividad en función de: (a) Variación de la profundidad de atacado respecto al valor de diseño, (b) Variación del factor de llenado respecto al valor de diseño y (c) Variación del periodo respecto al valor de diseño.

Por tanto, el DBR presenta una baja tolerancia frente a posibles desviaciones en fabricación, con lo cual se llevó a cabo un diseño más robusto para reducir la influencia de posibles variaciones debido a fabricación. La estrategia de diseño fue aumentar la anchura de la guía a 560nm. Además, los parámetros del DBR se ajustaron a valores múltiplos de 10, tal como se detalla en la tabla 3.7, para minimizar posibles desviaciones en fabricación. El espectro de funcionamiento obtenido por medio de simulación 3DFDTD con resolución de 30nm se muestra en la figura 3.41(a).

\begin{tabular}{|c|c|c|c|c|c|}
\hline \multirow[b]{2}{*}{$\begin{array}{l}\text { Parámetros de } \\
\text { diseño }\end{array}$} & \multirow[b]{2}{*}{$\begin{array}{l}\text { Diseño } \\
\text { Óptimo }\end{array}$} & \multicolumn{2}{|c|}{ Número de periodos $=33$} & \multicolumn{2}{|c|}{ Número de periodos $=60$} \\
\hline & & Reflectividad & $\begin{array}{c}\text { Ancho de Banda } \\
3 \mathrm{~dB}\end{array}$ & Reflectividad & $\begin{array}{c}\text { Ancho de Banda } \\
3 \mathrm{~dB}\end{array}$ \\
\hline Periodo & $240 \mathrm{~nm}$ & \multirow{3}{*}{$50 \%$} & \multirow{3}{*}{$30 \mathrm{~nm}$} & \multirow{3}{*}{$95 \%$} & \multirow{3}{*}{$20 \mathrm{~nm}$} \\
\hline Factor de llenado & $50 \%$ & & & & \\
\hline $\begin{array}{l}\text { Profundidad de } \\
\text { atacado }\end{array}$ & $30 \mathrm{~nm}$ & & & & \\
\hline
\end{tabular}

Tabla 3.7: Diseño mejorado del DBR considerando una guía de 560nm. Se detalla características del espectro (reflectividad y ancho de banda) obtenidos con el modelo de Matlab y simulaciones 3D-FDTD. 


\section{Capítulo 3: Diseño esquema de integración híbrida de láser con cavidad externa}

Finalmente, se realizó un análisis sobre variaciones en la anchura de la guía respecto al valor propuesto. En la figura 3.41(b) se observa que las variaciones en anchura de la guía no afectan a la reflectividad mientras que tiene un gran impacto respecto al desplazamiento del espectro del DBR (una anchura de la guía mayor al de diseño desplaza el espectro hacia longitudes de onda mayores). Sin embargo, el control de este parámetro en fabricación es mayor de tal manera que una variación de $\pm 10 \mathrm{~nm}$ provoca un desplazamiento del espectro de tan solo $\pm 3 \mathrm{~nm}$.

(a)

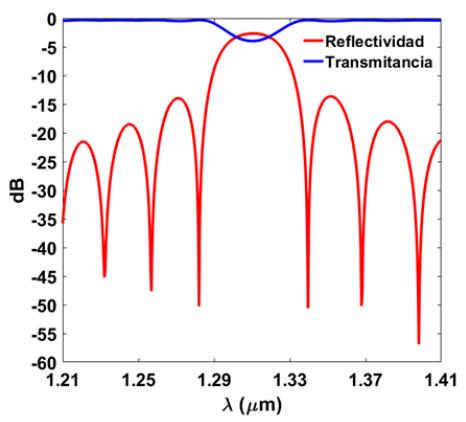

(b)

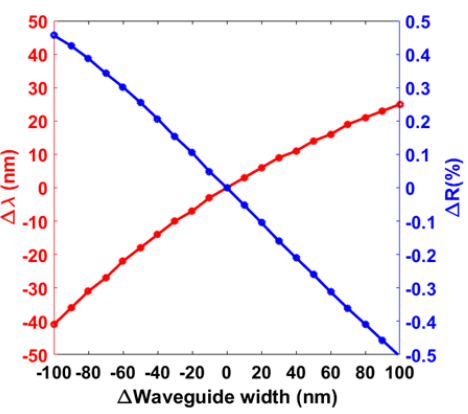

(c)

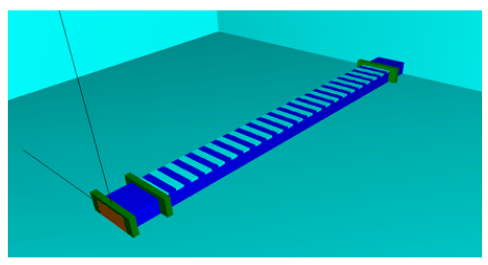

Figura 3.41. (a) Espectro de reflectividad y transmitancia del diseño mejorado considerando una guía de ancho 560nm y un número de periodos N=33, y (b) Desplazamiento del espectro de reflectividad del DBR y variación de la reflectividad en función de la variación del ancho de la guía, obtenido mediante simulaciones 3D-FDTD y resolución 30nm. (c) Esquemático de la estructura simulada.

\subsection{Diseño del mecanismo de sintonización termo-óptica}

Para la sintonización del láser de cavidad externa por medio del efecto termo-óptico, se analizó la inclusión de micro calentadores sobre el filtro de anillo y sobre una parte de la guía de la cavidad externa. Un incremento de temperatura sobre la guía del anillo resonante o sobre una sección de la guía de la cavidad permite la variación del índice de refracción $\Delta n$ del material respecto a su valor a temperatura ambiente $\left(20^{\circ} \mathrm{C}\right)$. Esta variación permite desplazar en longitud de onda la respuesta del filtro permitiendo así su sintonización, mientras que esa variación sobre una parte de la guía de la cavidad externa permite realizar una variación de la fase del modo propagado permitiendo ajustar la posición de los modos de la cavidad. En la figura 3.42 se muestra el espectro del filtro de anillo y el espectro de reflexión del DBR. Además, también se detallan los modos de cavidad obtenidos a partir de la condición de fase en el diseño del láser. Por una parte, la variación del índice de refracción en la guía del filtro de anillo y en el DBR desplaza la respuesta del espectro resultante en función del incremento de temperatura. Por otro lado, la variación de índice de refracción en una parte de la guía de la cavidad externa permite alinear los modos de la cavidad respecto a la resonancia del anillo e incrementar la potencia óptica de salida del láser. De esta manera, se realizó un análisis más detallado para determinar la variación del desplazamiento en longitud de onda en función del incremento de temperatura sobre cada estructura. 

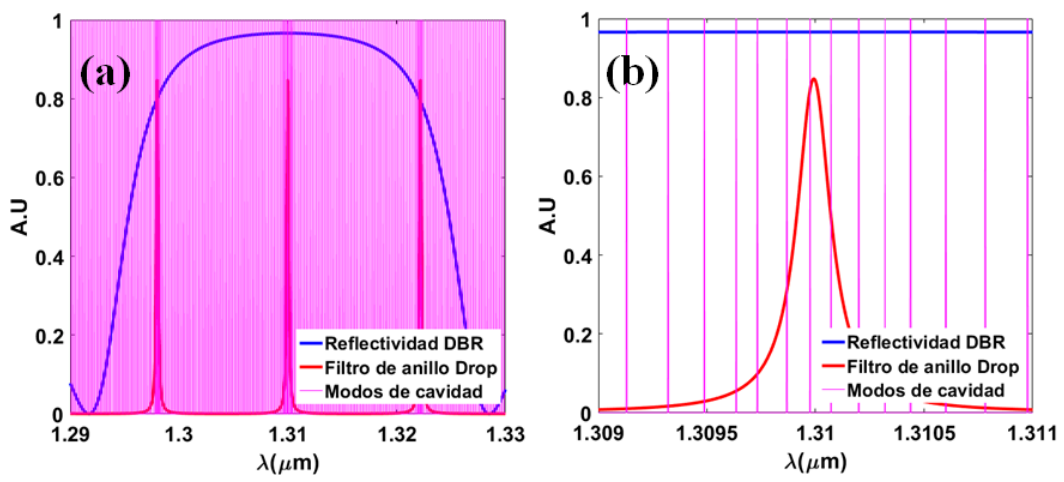

Figura 3.42. (a) Espectros de funcionamiento de las estructuras que forman la cavidad externa y (b) ampliación de la respuesta del filtro de anillo resonante y reflectividad del DBR, en ambos casos se detalla los modos de cavidad.

El análisis se llevó a cabo considerando el coeficiente termo-óptico del silicio y su variación respecto a la longitud de onda (figura 3.43(a)) siendo su valor de $\frac{d n}{d T}=$ $1.918 \times 10^{-4} \frac{1}{{ }^{\circ} \mathrm{C}}$ para $\lambda=1.31 \mu \mathrm{m}$. Aplicando un incremento de temperatura respecto a una temperatura ambiente de $20^{\circ} \mathrm{C}$, se estimó la variación del índice efectivo de la guía SOI con un espesor de $220 \mathrm{~nm}$ y una anchura de $500 \mathrm{~nm}$. La variación del índice efectivo $n e f f_{\text {Troom }}$ en función de la longitud de onda se muestra en la figura 3.43(b). Luego, utilizando el coeficiente termo-óptico, se calculó nuevamente el índice efectivo en la guía $n$ ef $f_{T}$ para un incremento de temperatura $T \geq T_{\text {room }}$. De esta manera, se calculó la variación del índice efectivo $\Delta n e f f=n e f f_{T}-n e f f_{\text {Troom }}$ y la variación de temperatura $\Delta T=T-$ $T_{\text {Troom }}$ (figura 3.43(c)), con los cuales se estimó una variación del índice efectivo respecto a la variación de temperatura de $\frac{\Delta n e f f}{\Delta T}=2.026 \times 10^{-4} \frac{1}{{ }^{\circ} C}$.
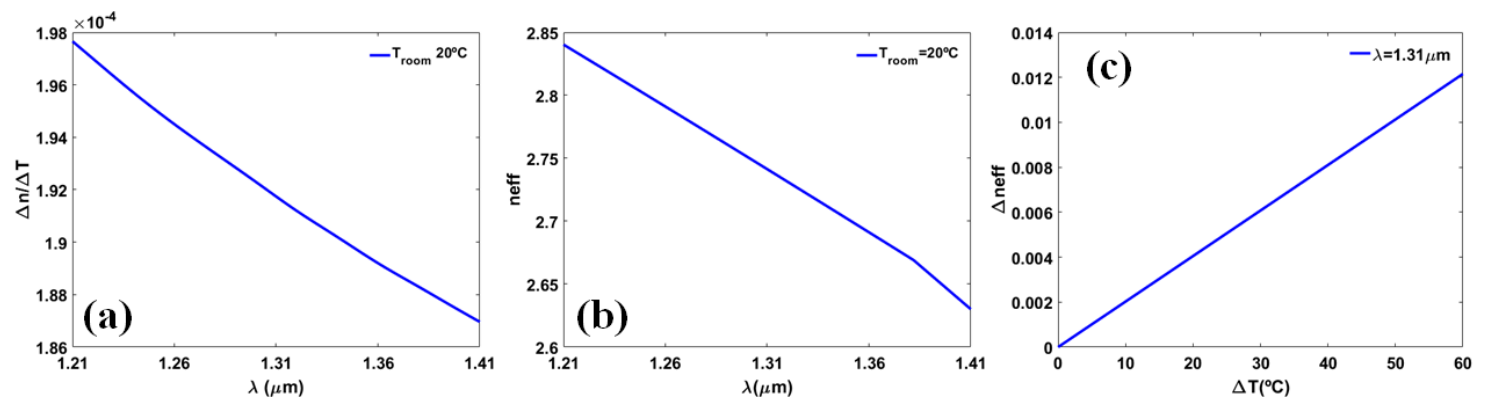

Figura 3.43. (a) Coeficiente termo-óptico del silicio en función de la longitud de onda. (b) Índice efectivo de una guía SOI de espesor $220 \mathrm{~nm}$ y anchura $500 \mathrm{~nm}$ calculado a temperatura ambiente mediante el método del índice efectivo. (c) Variación del índice efectivo obtenido a partir de un incremento de temperatura para una longitud de onda de $\lambda=1.31 \mu \mathrm{m}$.

Con la variación del índice efectivo respecto a la variación de temperatura, se calculó el desplazamiento del espectro en cada caso (figura 3.44(a)). En la figura 3.44(b) se muestra la respuesta del filtro de anillo además del desplazamiento de los modos de cavidad. En la figura 3.44(c) se muestra el desplazamiento del espectro de reflectividad del DBR, en el cual se verifica que el desplazamiento de dicho espectro debido a un incremento de temperatura no afecta en gran medida al funcionamiento del láser. 


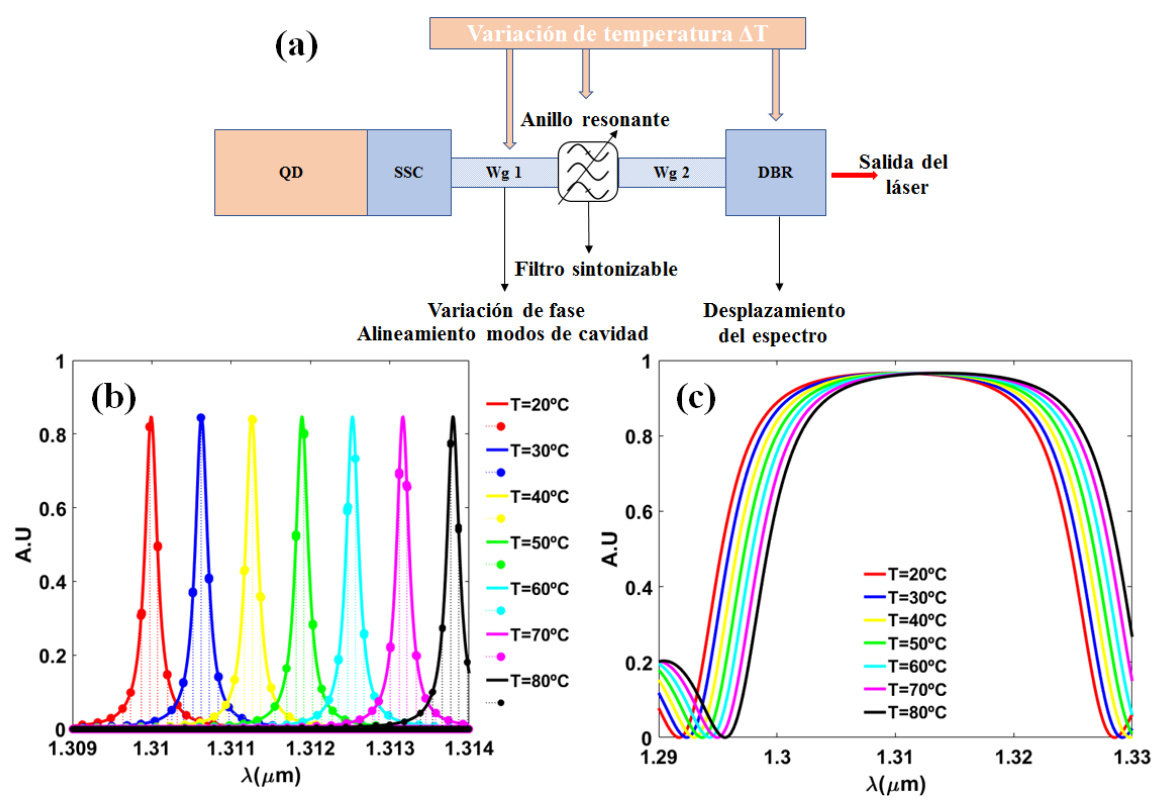

Figura 3.44. (a) Incremento de temperatura sobre una sección de guía de la cavidad externa, sobre el anillo resonante y sobre el DBR. (b) Espectro del filtro de anillo y desplazamiento de los modos de cavidad en función del incremento de temperatura. (c) Desplazamiento del espectro de reflectividad del DBR en función del incremento de temperatura.

A partir de los resultados obtenidos de desplazamiento debido al efecto termo-óptico de la respuesta del filtro y el espectro de reflectividad del DBR (figura 3.45(a)), se calculó la variación en longitud de onda , $\Delta \lambda=\lambda_{T}-\lambda_{\text {Troom }}$ siendo $\lambda_{\text {Troom }}=1.31 \mu \mathrm{m}$, frente a la variación de temperatura $\Delta T=T-T_{\text {Troom }}$ (figura 3.45(b)). De esta manera, se estimó una variación de $\frac{\Delta \lambda_{\text {ring }}}{\Delta T}=6.415 \times 10^{-2} \frac{\mathrm{nm}}{{ }^{\circ} \mathrm{C}}$ para el filtro y una variación de $\frac{\Delta \lambda_{D B R}}{\Delta T}=$ $6.432 \times 10^{-2} \frac{\mathrm{nm}}{{ }^{\circ} \mathrm{C}}$ para el DBR.

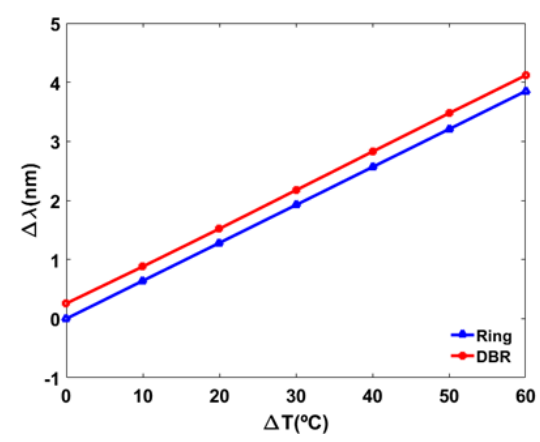

Figura 3.45. Desplazamiento de la respuesta del filtro de anillo y DBR respecto a la longitud de onda a la que se encuentran centrados en función de la variación de temperatura.

Dichos valores son similares, lo que implica que la sintonización del láser por medio del efecto termo-óptico solo requiere ser aplicada sobre el filtro de anillo resonante y una sección de la guía de la cavidad externa (figura 3.46(a)), sin que éste se vea afectado al no aplicarlo sobre el DBR. La figura 3.46(b) muestra el espectro de salida del láser sintonizable. 

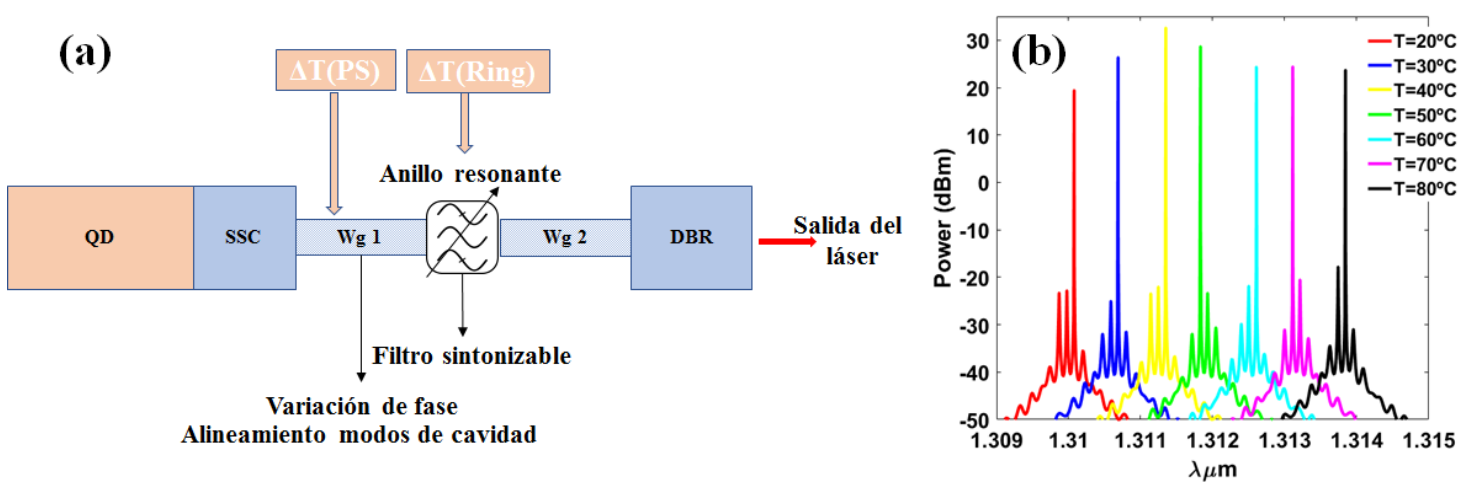

Figura 3.46. (a) Esquema del láser de cavidad externa con sintonización en el filtro de anillo y sección de guía de la cavidad externa mediante el efecto termo-óptico. (b) Espectro de salida del láser sintonizable.

\subsection{Diseño del acoplador a fibra basado en red de difracción}

Para el diseño se consideró una longitud de onda $\lambda=1310 \mathrm{~nm}$ y polarización TE, de tal manera que los índices de refracción de los materiales que constituyen la estructura, silicio y óxido son: $\mathrm{Si}=3.507$ y $\mathrm{SiO}_{2}=1.446$. Por otro lado, el espesor de la capa de silicio es de $L_{S i}=220 \mathrm{~nm}$ y de la capa de óxido de $L_{B o x}=2 \mu \mathrm{m}$. Inicialmente se consideró una capa superior de óxido de $L_{\text {cover }}=750 \mathrm{~nm}$. En la figura 3.47 se muestra el corte longitudinal de la estructura de acoplo, en la cual se detalla todos los parámetros a tener en cuenta para su diseño.

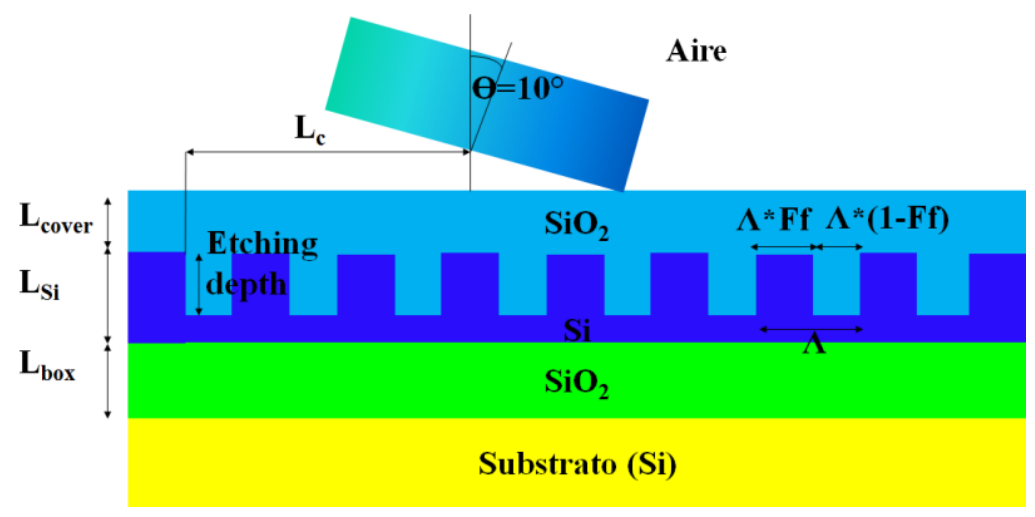

Figura 3.47: Corte longitudinal de la estructura de acoplo indicando los parámetros de diseño. Parámetros de la guía referentes al espesor de las capas de: óxido $\left(L_{c o v e r}, L_{b o x}\right)$, silicio $\left(L_{S i}\right)$. Parámetros de la red de difracción: periodo del grating ( $($ ), Factor de llenado $(F f)$, Profundidad de atacado (Etching depth). Parámetros de alineamiento de la fibra y el grating: Ángulo de incidencia $\left(\theta=10^{\circ}\right)$, Longitud de acoplo $\left(L_{c}\right)$.

La fibra óptica monomodo con la cual se acopla luz al acoplador se ubica sobre la superficie de la red de difracción, con un ángulo de incidencia $\theta=10^{\circ}$, y a una distancia respecto al inicio del grating $L c$, cuyo valor se obtiene a partir de la expresión mostrada 


\section{Capítulo 3: Diseño esquema de integración híbrida de láser con cavidad externa}

en el capítulo 2 (Eq. 2.1), siendo el diámetro del campo modal MFD=9.2 $\mu \mathrm{m}$ para la longitud de onda de 1310nm.

Inicialmente se asumió un $F f=50 \%$, y dada una profundidad de ataque, se calculó el índice efectivo de la guía de la red de difracción $\left(n e f f_{g}\right)$ por medio de la Eq. 3.31 [100], en la cual nef $f_{\text {nup }}$ y nef $f_{\text {nep }}$ representan los índices efectivos de la parte que no se ataca y de la parte atacada respectivamente, tal como se observa en la figura 3.48.

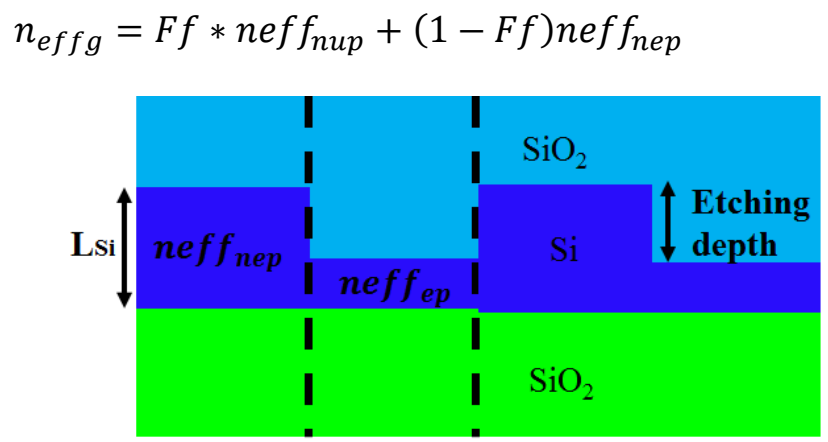

Figura 3.48: Estructura de la red de difracción formada por una parte cuyo núcleo de la guía (Si) no se ataca y otra parte que si se ataca (Etching depth). De esta manera el índice efectivo del modo propagado en la guía es modulado acorde a una función periódica de valores neff nep-y neffep.

Con la utilización de la ecuación de Bragg introducida en el capítulo 1 (Eq. 1.2), y haciendo una variación en la profundidad de atacado, se determinó de manera inicial el periodo de la red de difracción para cada profundidad de ataque. Con los valores obtenidos, se realizaron varias simulaciones 2D-FDTD para determinar la profundidad de atacado óptima, siendo esta de 70nm (figura 3.49(a)). Posteriormente, también mediante simulaciones 2D-FDTD, se optimizó el valor del periodo y el factor de llenado, siendo el óptimo $\Lambda=485 \mathrm{~nm}$ y $F f=53 \%$ (figura 3.49 (b)). Finalmente se realizaron varias simulaciones haciendo una variación del espesor de las capas de $\mathrm{SiO}_{2}\left(L_{c o v e r}, L_{B o x}\right)$. Los resultados muestran que la variación de dichos parámetros corresponde a una función periódica con eficiencias de acoplo máximas y mínimas, debido a una interferencia constructiva y destructiva, que se repiten en función del espesor (figura 3.49(c)). Los parámetros óptimos del diseño se detallan en la tabla 3.8.
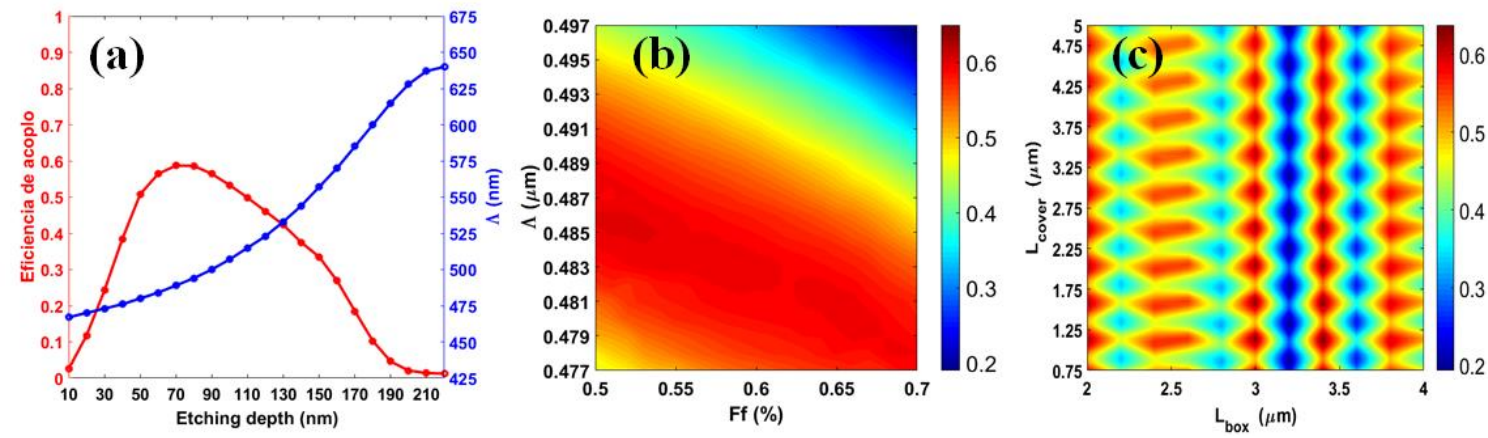

Figura 3.49: Simulaciones 2D-FDTD con resolución de 30nm: (a) Eficiencia de acoplo y periodo de la red de difracción en función de la profundidad de ataque, (b) Optimización del periodo y factor de llenado, (c) Variación del espesor de las capas de $\mathrm{SiO}_{2}$ de la estructura, $L_{b o x}$ y $L_{c o v e r}$. 


\subsection{Diseño del acoplador a fibra basado en red de difracción}

\begin{tabular}{|c|c|}
\hline $\begin{array}{c}\text { Parámetros de } \\
\text { diseño }\end{array}$ & Diseño óptimo \\
\hline $\begin{array}{c}\text { Periodo de la red } \\
\text { de difracción }\end{array}$ & $485 \mathrm{~nm}$ \\
\hline $\begin{array}{c}\text { Factor de llenado } \\
\text { Espesor de la } \\
\text { cubierta de óxido }\end{array}$ & $53 \%$ \\
\hline
\end{tabular}

Tabla 3.8: Parámetros de diseño óptimos del acoplador a fibra óptica por medio de red de difracción a una guía SOI de 500nm de ancho y 220nm de espesor.

De manera similar a la estructura de acoplo diseñada en el capítulo 2, los parámetros óptimos del acoplador lineal se usaron en el diseño de una red de difracción basada en elipses confocales (figura 3.50).
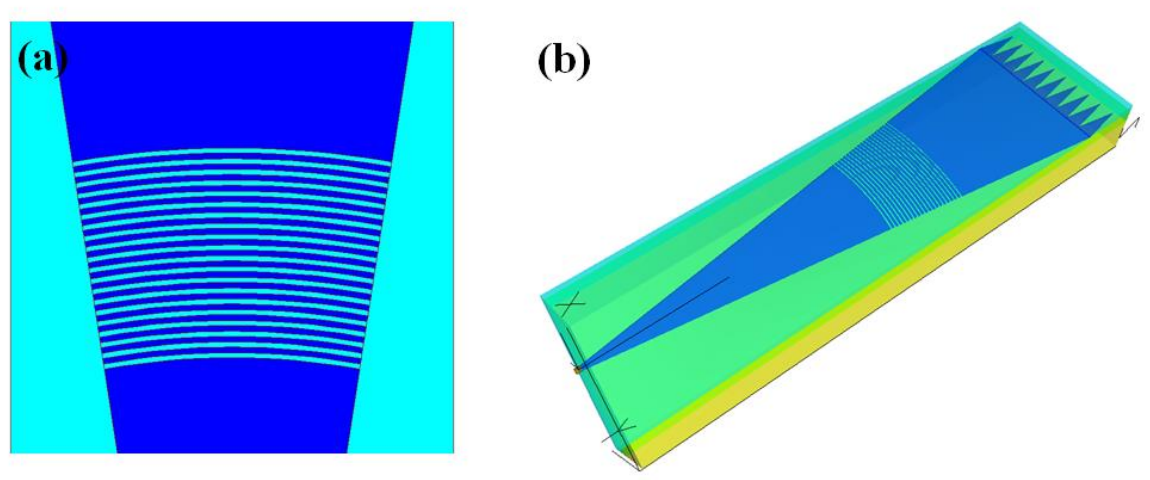

Figura 3.50: (a) Acoplador a fibra óptica basado en red de difracción de elipses confocales. (b) Estructura 3D del acoplador a fibra mediante red de difracción de elipses confocales.

Por último, se realizó una simulación 3D-FDTD para evaluar el funcionamiento del acoplador a fibra diseñado y determinar el espectro resultante cuya máxima eficiencia de acoplamiento es de alrededor del $52 \%$, centrado entorno a $\lambda=1310 \mathrm{~nm}$ y cuyo ancho de banda de acoplamiento medido a $3 \mathrm{~dB}$ es de $63 \mathrm{~nm}$, tal como se observa en la figura 3.51 .

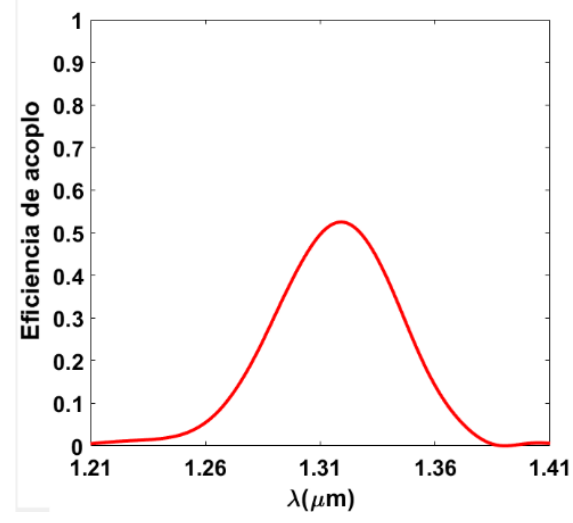

Figura 3.51: Simulación 3D-FDTD con resolución de 30nm para obtener el espectro de acoplamiento del grating curvo a partir de elipses confocales, obteniendo una eficiencia de acoplo máxima del 52\%. 


\subsection{Diseño del acoplador a fibra basado en convertidor de tamaño de modo}

El diseño del acoplador se realizó mediante simulaciones 3D-BPM para una longitud de onda $\lambda=1310 \mathrm{~nm}$ y polarización TE y con el objetivo principal de minimizar las pérdidas de acoplo. La estructura del acoplador es la misma que la presentada en la sección 3.2. Primero se optimizó la longitud del taper y el ancho de punta. En este caso se asumió de manera inicial una variación lineal del taper y un espesor de $4 \mu \mathrm{m}$ para la capa de óxido que cubre el acoplador con el fin de tener unas dimensiones en altura similares al radio del núcleo de la fibra monomodo estándar. En este caso los valores óptimos del taper invertido son: $L t=500 \mu \mathrm{m}$ y $T w=60 \mathrm{~nm}$ (figura $3.52(\mathrm{a})$ ). Posteriormente, se hizo una variación de los parámetros de la cubierta de óxido, siendo óptimos los valores: $W_{\text {cover }}=8 \mu \mathrm{m}$ y $L_{\text {cover }}=3.5 \mu \mathrm{m}$, alcanzando unas pérdidas de acoplo de $1.7 \mathrm{~dB}$ (figura 3.52(b)). Finalmente, se hizo una variación con respecto al espesor de la capa de óxido $L_{b o x}$ reduciendo las pérdidas de acoplo a $0.9 \mathrm{~dB}$ para un espesor de esta capa de $3 \mu \mathrm{m}$ (figura 3.52(c)).
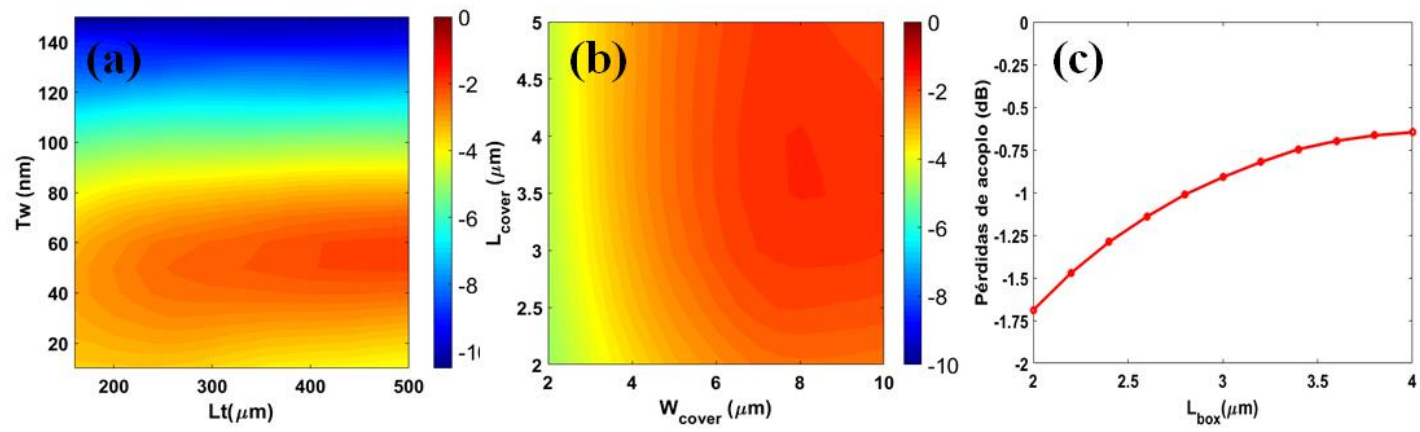

Figura 3.52: Simulaciones 3D-BPM con resolución de 50nm para la obtención de pérdidas de acoplo (dB) en función de: (a) longitud del taper ( $L t)$ y el ancho de la punta $(T w)$, (b) espesor y ancho de la cubierta de óxido $\left(L_{c o v e r}, W_{\text {cover }}\right)$ y (c) espesor de la capa de óxido $\left(L_{b o x}\right)$.

Con los parámetros de diseño óptimos se procedió a obtener el espectro de funcionamiento del acoplador, del cual se observa un ancho de banda de acoplo bastante amplio y casi en su totalidad plano, tal y como se muestra en la figura 3.53.

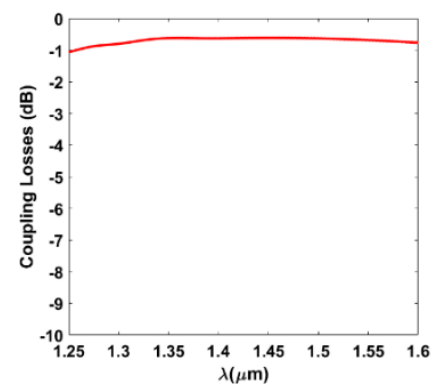

Figura 3.53: Simulación 3D-BPM con resolución 50nm para obtener el espectro de acoplamiento a fibra del acoplador mediante un convertidor de tamaño de modo obteniendo unas pérdidas de acoplo de $0.9 \mathrm{~dB}$ en polarización TE. 
El acoplamiento horizontal o de borde requiere de una gran precisión a nivel de alineamiento, por lo cual se realizó un estudio de tolerancia frente al desalineamiento entre la fibra y el conversor de tamaño de modo diseñado. De manera inicial, se calcularon las pérdidas respecto a la posición de la fibra óptica en el plano transversal considerando una distancia entre la fibra y el acoplador de gap=0 (figura 3.54(a)). Se observa una tolerancia de $\pm 0.5 \mu \mathrm{m}$ en la posición de la fibra óptica para asegurar unas pérdidas por desalineamiento menores a $1 \mathrm{~dB}$. Posteriormente, se calcularon las pérdidas respecto a la distancia gap (eje de propagación z) entre la fibra y el convertidor de tamaño de modo (figura 3.54(b)). En este caso, la variación permitida debe ser menor a $10 \mu \mathrm{m}$ para asegurar unas pérdidas por desalineamiento menores a $1 \mathrm{~dB}$.

(a)

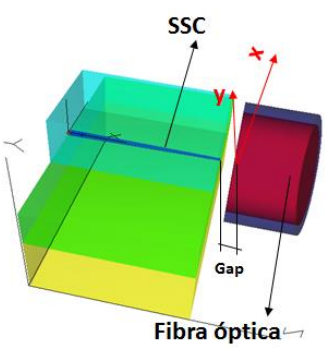

(b)

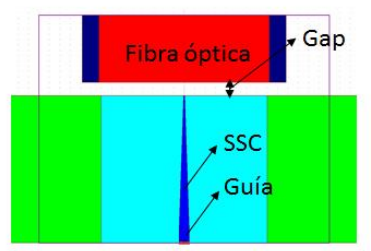

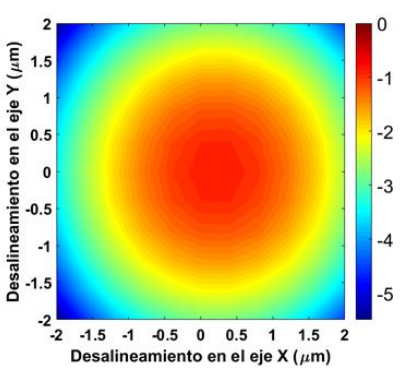
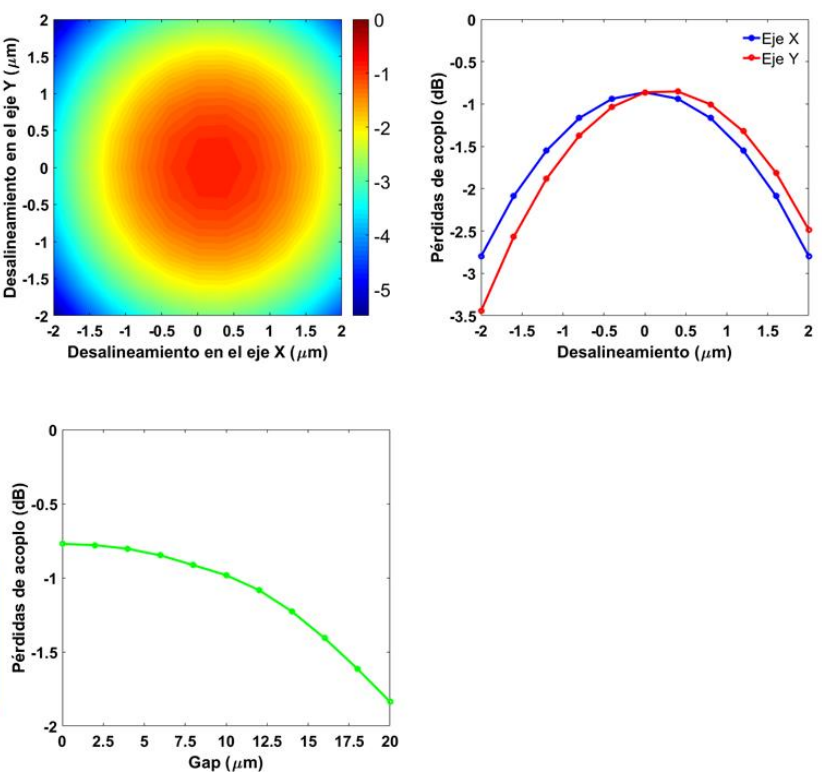

Figura 3.54: Simulación 3D-BPM con resolución 50nm para analizar la tolerancia frente al desalineamiento entre la fibra óptica y el conversor de tamaño de modo diseñado. (a) Pérdidas debidas al desplazamiento de la fibra en el plano transversal. (b) Pérdidas debido a variaciones en la distancia entre la fibra y el acoplador.

De igual manera que en el diseño del acoplador al QD, el convertidor de tamaño de modo para acoplo a fibra se optimizó a nivel de longitud total de la estructura usando un taper con perfil parabólico, tal como se detalla en la figura 3.55.

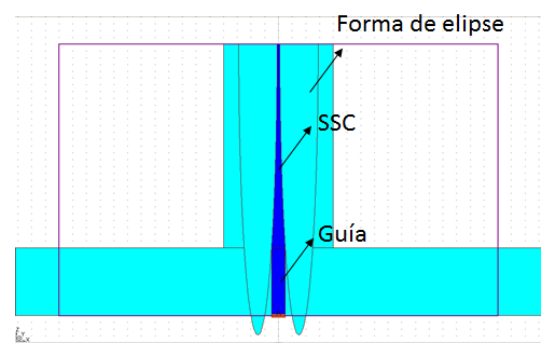

Figura 3.55: Estructura del convertidor de tamaño de modo basado en un taper invertido con perfil parabólico. 


\section{Capítulo 3: Diseño esquema de integración híbrida de láser con cavidad externa}

Los parámetros de diseño de la nueva estructura se optimizaron mediante simulaciones 3D-BPM. De este modo, a partir de los valores de espesor de las capas de óxido y ancho de la cubierta obtenidos previamente $\left(L_{b o x}=3 \mu \mathrm{m}, L_{\text {cover }}=3.5 \mu \mathrm{m}\right.$ y $\left.W_{\text {cover }}=8 \mu \mathrm{m}\right)$, se optimizó la longitud del taper y el ancho de punta, reduciendo el valor de la longitud del taper a $L t=130 \mu \mathrm{m}$ y aumentado el valor del ancho de punta a $T w=70 \mathrm{~nm}$ (figura 3.56(a)). A continuación, se optimizó el espesor y la anchura de la cubierta de óxido, siendo óptimos los mismos valores con los cuales se inició la optimización $L_{\text {cover }}=3.5 \mu \mathrm{m}$ y $W_{\text {cover }}=8 \mu \mathrm{m}$ (figura 3.56(b)). Finalmente, se confirmó que el valor óptimo de la capa de óxido es $\mathrm{L}_{\text {box }}=3 \mu \mathrm{m}$ alcanzando unas pérdidas de acoplo de 0.6dB (figura 3.56(c)).
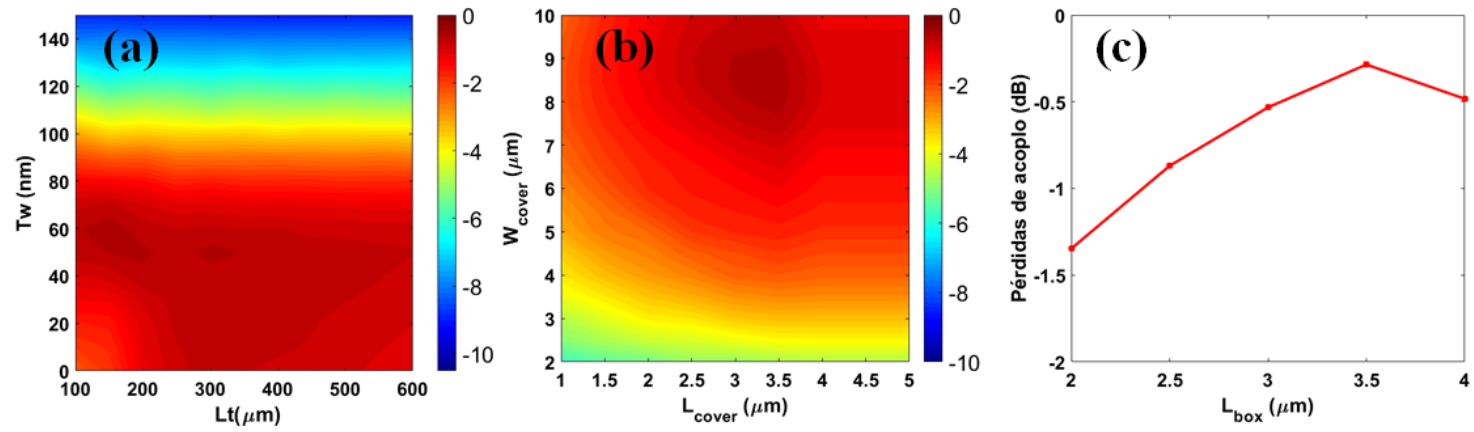

Figura 3.56: Simulaciones BPM 3D con resolución de 50nm. Pérdidas de acoplo (dB) en función de: (a) longitud del taper ( $L t)$ y el ancho de la punta $(T w)$, (b) espesor y ancho de la cubierta de óxido $\left(L_{c o v e r}, W_{\text {cover }}\right)$, y (c) espesor de la capa de óxido $\left(L_{b o x}\right)$.

Cabe destacar que el valor del espesor de la cubierta de óxido difiere de la óptima en el diseño del acoplador a QD, siendo éste de mayor prioridad para la integración de la cavidad externa. Por tanto, el valor de este parámetro se fijó en $L_{c o v e r}=2 \mu \mathrm{m}$. Una vez fijado este valor se optimizó nuevamente la anchura de la cubierta de óxido $W_{\text {cover }}=9 \mu \mathrm{m}$, alcanzado unas pérdidas de acoplo de $1.14 \mathrm{~dB}$. Los parámetros finales del diseño se muestran en la tabla 3.9.

\begin{tabular}{|c|c|}
\hline $\begin{array}{c}\text { Parámetros de } \\
\text { diseño }\end{array}$ & Diseño final \\
\hline $\begin{array}{c}\text { Longitud del SSC } \\
\text { Ancho de la parte } \\
\text { estrecha del SSC }\end{array}$ & $130 \mu \mathrm{m}$ \\
\hline $\begin{array}{c}\text { Ancho de la } \\
\text { cubierta de óxido }\end{array}$ & $70 \mathrm{~nm}$ \\
\hline $\begin{array}{c}\text { Espesor de la } \\
\text { cubierta de óxido }\end{array}$ & $9 \mu \mathrm{m}$ \\
\hline
\end{tabular}

Tabla 3.9: Parámetros de diseño finales del acoplador a fibra óptica.

Finalmente, se calculó el espectro de la estructura optimizada mediante simulaciones 3D-BPM y 3D-FDTD, comprobando nuevamente un amplio ancho de banda de funcionamiento (figura 3.57(a)). Además, se realizó nuevamente un análisis de pérdidas de acoplo por desalineamiento mediante simulaciones 3D-BPM, obteniendo unas tolerancias similares a los valores anteriormente obtenidos (figura 3.57(b, c)). 
3.7 Diseño del acoplador a fibra basado en convertidor de tamaño de modo
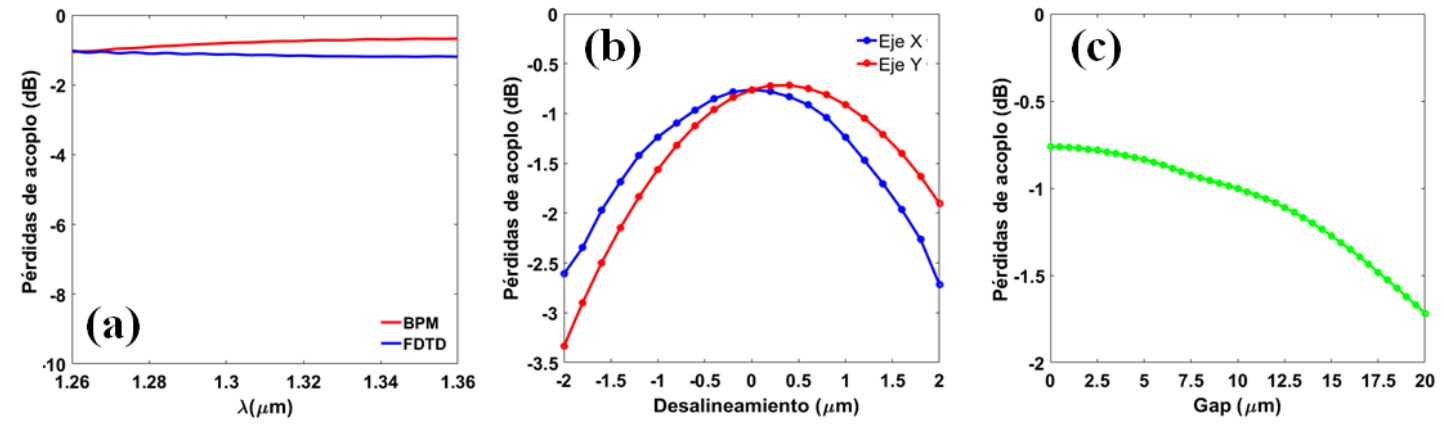

Figura 3.57: Simulaciones 3D-BPM y 3D-FDTD con resolución 50nm para obtener: (a) el espectro de acoplamiento, y la tolerancia en pérdidas de alineamiento debido a: (b) desplazamiento de la fibra óptica en el plano transversal y (c) distancia entre la fibra óptica y el acoplador. 



\section{Capítulo 4}

\section{Fabricación y resultados experimentales del esquema propuesto de integración híbrida de láser con cavidad externa}

\subsection{Descripción del montaje de medidas}

La caracterización de las muestras fabricadas exige acoplar luz proveniente de una fuente externa por lo cual es esencial un acoplo eficiente entre la fibra óptica y el chip de silicio. Para realizar las medidas se utilizaron varios montajes. Por un lado, se usó un montaje de acoplo horizontal para caracterizar el acoplador basado en convertidor de tamaño de modo. Por otro lado, se utilizó un montaje de acoplo vertical para caracterizar el acoplador por red de difracción. Además de la evaluación de las estructuras de acoplo tanto de manera horizontal como vertical, se caracterizaron otras estructuras en el chip de silicio, como el filtro de anillo resonante y el reflector DBR. Finalmente, se empleó un banco de alineamiento con una mayor precisión de movimiento en todos los ejes (x, y, z) para la caracterización del láser de cavidad externa. De esta forma, se llevó a cabo un alineamiento activo entre el chip de ganancia QD, el chip de silicio y la fibra óptica de salida. 
Capítulo 4: Fabricación y resultados experimentales del esquema propuesto de integración híbrida de láser con cavidad externa

\subsubsection{Montaje para el acoplamiento horizontal}

El esquema del montaje para acoplo horizontal se muestra en la figura 4.1(a). Esta configuración permite alinear una fibra óptica monomodo a una lente u objetivo. La emisión de luz en la fibra óptica proviene de una fuente de luz externa (láser sintonizable Yenista T100) controlado vía software (LabVIEW) mediante ordenador. El alineamiento a objetivo permite colimar el haz de luz del puerto de salida del dispositivo bajo prueba (DBP), refiriéndonos al chip de silicio, a una cámara de infrarrojo pasando antes por un divisor de potencia del 50\%. De esta manera, una parte del haz de luz es proyectado a la cámara de infrarrojo que se conecta al ordenador y en tiempo real muestra una imagen de del haz de salida mientras que la otra parte del haz es detectada por un fotodetector conectado a un medidor de potencia (Power Meter Ando AQ2140). Cuanto mayor sea la potencia óptica detectada mejor alineada estará la fibra de entrada. En una segunda etapa, tras una pequeña modificación del montaje (figura 4.1(b)), se alineará una fibra óptica de salida conectada al fotodetector. De manera similar cuanto mayor sea la potencia de luz captada en el fotodetector, mejor será el alineamiento fibra a fibra.

(a)

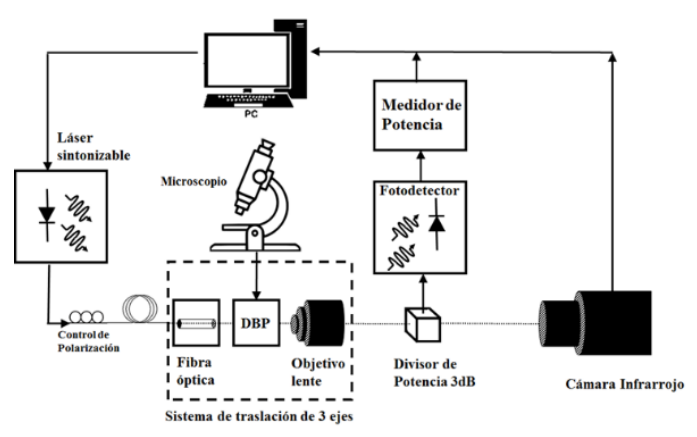

(b)

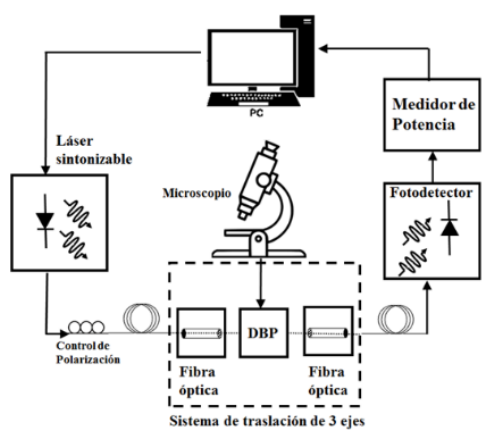

Figura 4.1: Esquema del montaje para acoplamiento horizontal, en una etapa inicial para (a) alineamiento de una fibra a objetivo, y en una siguiente etapa para (b) alineamiento fibra a fibra.

En la figura 4.2(a) se muestran fotografías del montaje para acoplo horizontal, en la cual se detalla el alineamiento fibra a objetivo (figura 4.2(b)), y la configuración de salida compuesta por un divisor de potencia a $3 \mathrm{~dB}$ que divide una parte del haz de luz a una cámara de infrarrojo y la otra al fotodetector (figura 4.2(c)).

(a)

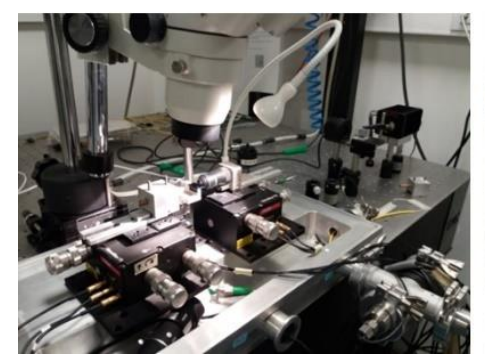

(b)

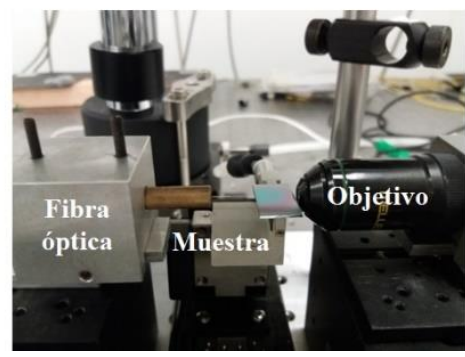

(c)

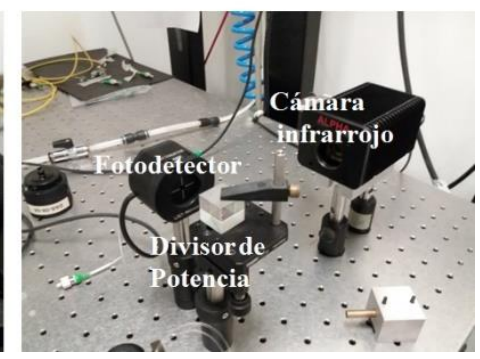

Figura 4.2: (a) Montaje para medidas en acoplo horizontal. (b) Alineamiento fibra a objetivo y (c) configuración de salida. 


\subsubsection{Montaje para el acoplamiento vertical}

El esquema del montaje para acoplo vertical se muestra en la figura 4.3. Al igual que en la configuración para acoplo horizontal, la luz emitida por el láser sintonizable externo pasa por un polarizador antes de inyectarse en el dispositivo bajo prueba (chip de silicio). La fibra óptica está colocada a un ángulo de inclinación de $10^{\circ}$ respecto a la normal de la superficie del chip de silicio y se alinea al puerto de entrada compuesto por la red de difracción. Una vez acoplada la luz al chip, otra fibra óptica dispuesta a la misma inclinación que la fibra de entrada y alineada al puerto de salida, recoge la luz proveniente de la red de difracción de salida, acoplando luz del chip a la fibra. De esta manera la luz acoplada a la fibra de salida es detectada por el fotodetector y su potencia óptica medida con el medidor de potencia (Thorlabs PM320E). El alineamiento se optimiza mediante un sistema de traslación de 3 ejes provisto tanto para la fibra de entrada como para la de salida maximizando la potencia óptica de salida detectada en el fotodetector.

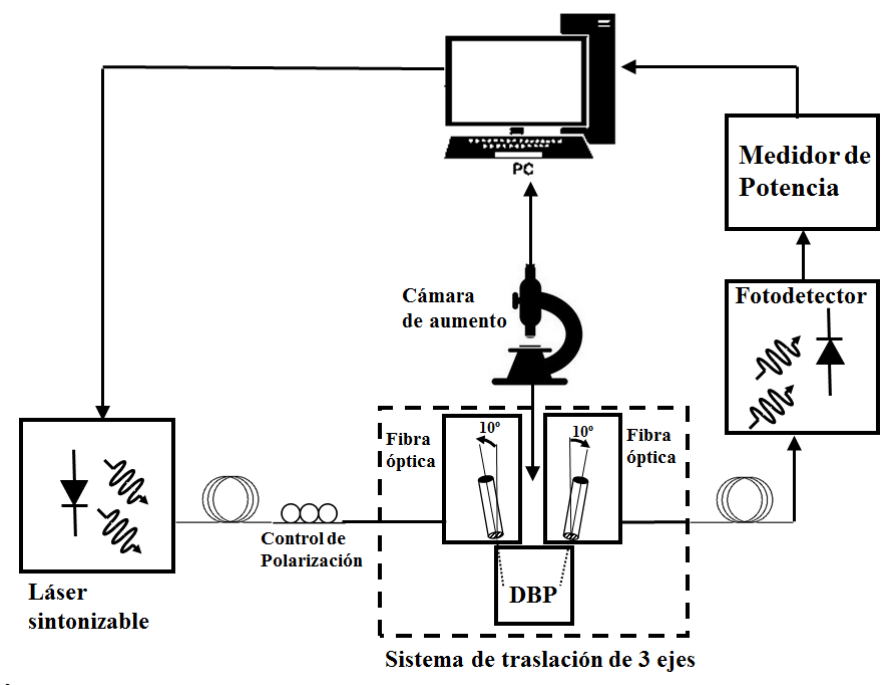

Figura 4.3: Esquema del montaje para acoplamiento vertical.

En la figura 4.4(a) se muestran fotografías del montaje para acoplo vertical utilizado, en la cual se detalla el alineamiento de la fibra de entrada y salida colocadas a un ángulo de inclinación de $10^{\circ}$ respecto a la normal de la superficie del chip de silicio (figura 4.4(b)).

(a)

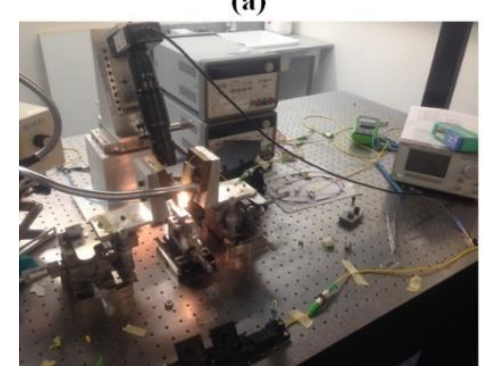

(b)

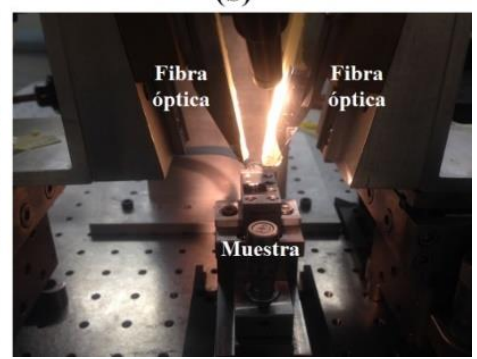

Figura 4.4: (a) Montaje para medidas en acoplo vertical. (b) Alineamiento de fibra óptica de entrada y salida respecto a la muestra bajo medida. 
Capítulo 4: Fabricación y resultados experimentales del esquema propuesto de integración híbrida de láser con cavidad externa

\subsubsection{Banco de alineamiento}

El alineamiento activo entre el chip de ganancia QD y el chip de silicio se realizó mediante un banco de alineamiento para conseguir una mejor precisión en el esquema de acoplo horizontal. El banco de alineamiento dispone de los mismos elementos que el montaje de acoplo horizontal descrito previamente, tanto para la configuración de alineamiento con objetivo (figura 4.5(a)) como de alineamiento con fibra óptica de salida (figura 4.5(b)). Sin embargo, la principal ventaja es su sistema de traslación de tres ejes con resolución de hasta 100nm y movimiento controlado vía software por una consola de mando, además de proveer un sistema de cámaras de aumento de alta resolución, una de vista superior a la muestra, y otra de vista lateral. De esta manera, se tiene un control mucho más preciso y estable del alineamiento.

(a)

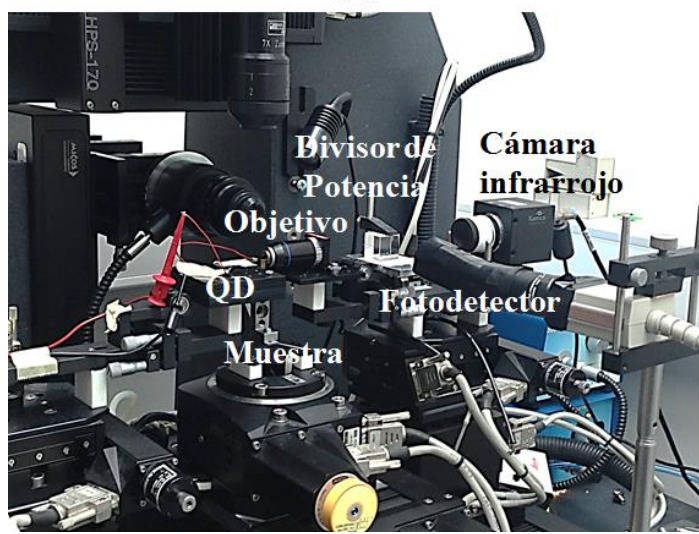

(b)

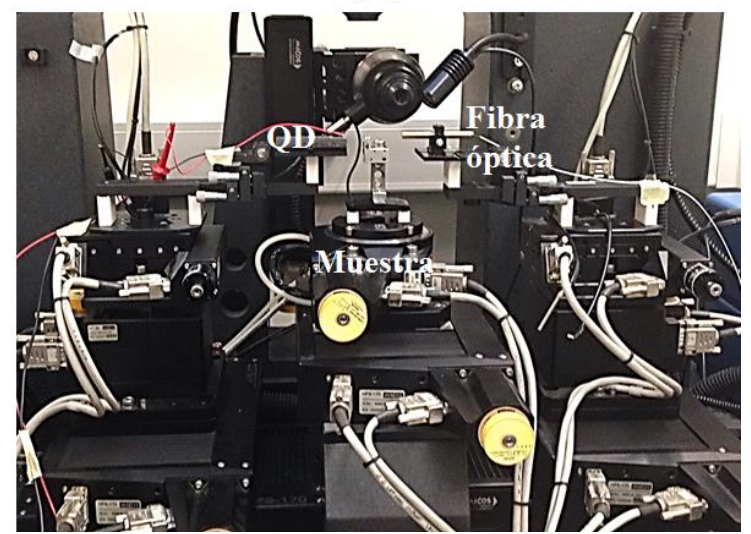

Figura 4.5: Banco de alineamiento con montaje para acoplamiento horizontal para (a) alineamiento a objetivo y (a) alineamiento a una fibra óptica de salida.

\subsection{Fabricación y resultados experimentales de bloques básicos}

El proceso de fabricación parte de una muestra de silicio sobre aislante (SOI) estándar con una capa de silicio en la parte superior de $220 \mathrm{~nm}$ de espesor (resistividad $\rho \sim 10 \Omega \mathrm{cm}^{-1}$ con un ligero dopado-p de $\sim 10^{15} \mathrm{~cm}^{-1}$ ) y un espesor de la capa BOX de $3 \mu \mathrm{m}$. La fabricación de las estructuras comienza con un proceso de litografía por haz de electrones (e-beam) sobre una resina de 100nm de hidrógeno silsesquioxane (HSQ). La HSQ es una resina negativa lo cual implica que el patrón a grabar es expuesto directamente. Después de revelar la resina HSQ, utilizando TMAH como revelador, los patrones de la resina son transferidos a las muestras SOI empleando un proceso optimizado de grabado de iones reactivos por plasma acoplado inductivamente (ICP-RIE) con gases de flúor $\left(S F_{6} / C_{4} F_{8}\right)$. Ambos gases son inyectados al mismo tiempo bajo condiciones de plasma óptimas para el grabado de silicio que produce un ataque anisotrópico con paredes laterales lisas. Además, el proceso de ataque se optimiza para 
alcanzar la profundidad requerida dependiendo del tipo de estructura (220nm para las guías, 70nm para la red de difracción o 30nm para los DBRs). Los pasos básicos de proceso se muestran en la figura 4.6. Una vez se ha grabado el silicio, la muestra se cubre con una capa de $\mathrm{SiO}_{2}$ mediante un proceso de depósito químico de vapor asistido por plasma (PECVD).

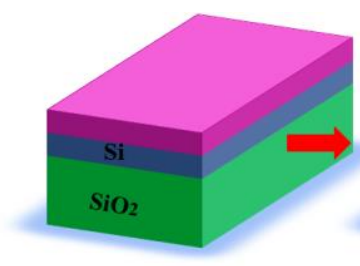

(1)

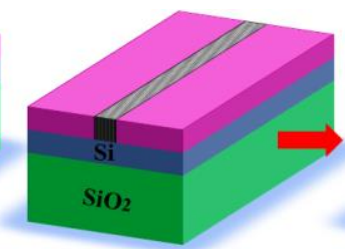

(2)

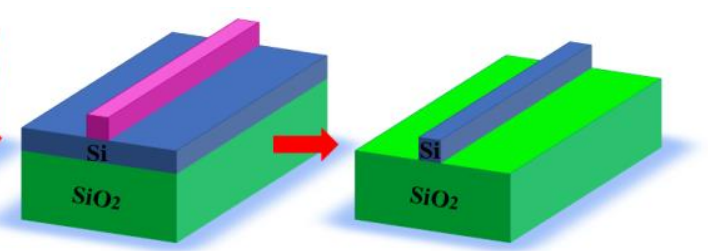

(3)
(4) - (5)

Figura 4.6: Pasos básicos del proceso de fabricación.

\subsubsection{Caracterización del acoplador mediante red de difracción}

Para la caracterización del acoplador mediante red de difracción se elaboró un archivo con la máscara (GDS). La máscara está basada en un conjunto de guías de anchura 500nm y varias longitudes con redes de difracción de entrada y salida, tal como se puede observar en la figura 4.7(a). Una vez fabricada la muestra, se realizaron varias imágenes SEM (Microscopio electrónico de barrido), como se muestra en la figura 4.7(b).

(a)
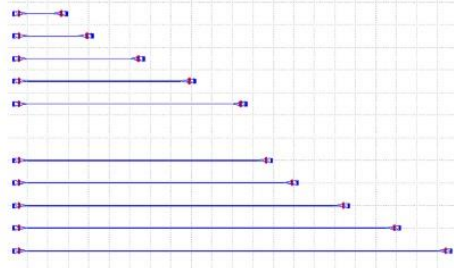

(b)

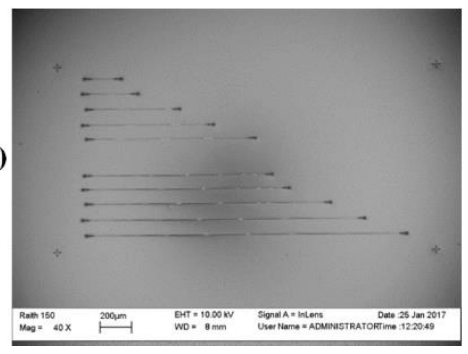

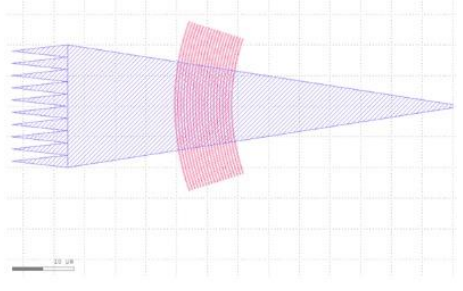

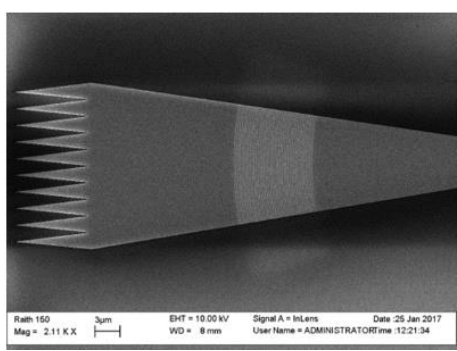

Figura 4.7: (a) GDS de la estructura para acoplamiento vertical por medio de una red de difracción, y (b) imágenes SEM de la muestra fabricada.

Después de realizar las medidas, se analizaron los datos experimentales por medio de un balance de potencias (Eq.4.1) para determinar las pérdidas de acoplo. Las pérdidas de propagación en las guías se estimaron mediante una regresión lineal de la máxima potencia óptica obtenida para cada longitud, obteniéndose unas pérdidas de propagación de $\alpha_{w g} @ 1310 \mathrm{~nm}=4.7 \frac{\mathrm{dB}}{\mathrm{cm}}$. De esta manera, las pérdidas de acoplo estimadas son de $\alpha_{g} @ 1310 \mathrm{~nm}=4.5 \mathrm{~dB}$. El espectro normalizado de pérdidas de acoplo obtenido tanto en 
Capítulo 4: Fabricación y resultados experimentales del esquema propuesto de integración híbrida de láser con cavidad externa

caracterización como mediante simulación 3D-FDTD se muestra en la figura 4.8. El ancho de banda medido a $3 \mathrm{~dB}$ es $B W_{3 d B}=54 \mathrm{~nm}$.

$$
\alpha_{g}[d B]=\frac{1}{2}\left(P_{\text {in }}[d B m]-P_{\text {out }}[d B m]-\alpha_{w g} L_{w g}[d B]\right)
$$

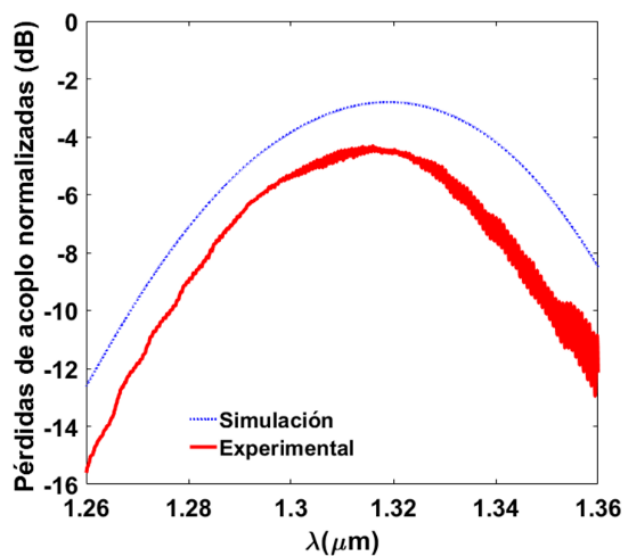

Figura 4.8: Espectro de pérdidas de acoplo experimentales y simuladas mediante 3D-FDTD.

\subsubsection{Caracterización del acoplador de tamaño de modos a fibra}

La caracterización de la estructura de acoplo basado en el convertidor de tamaño de modo se realizó mediante la fabricación de una muestra con varias guías de anchura 500nm y longitud de 5mm, distancia mínima para realizar el corte manual de la muestra. El GDS de la muestra se observa en la figura 4.9(a) en la que se detalla el convertidor de tamaño de modo y la muestra con varias guías. Después de ser fabricadas, se tomaron varias imágenes SEM de la estructura de acoplo para comprobar que las dimensiones correspondían a las de diseño (figura 4.9(b)).

(a)
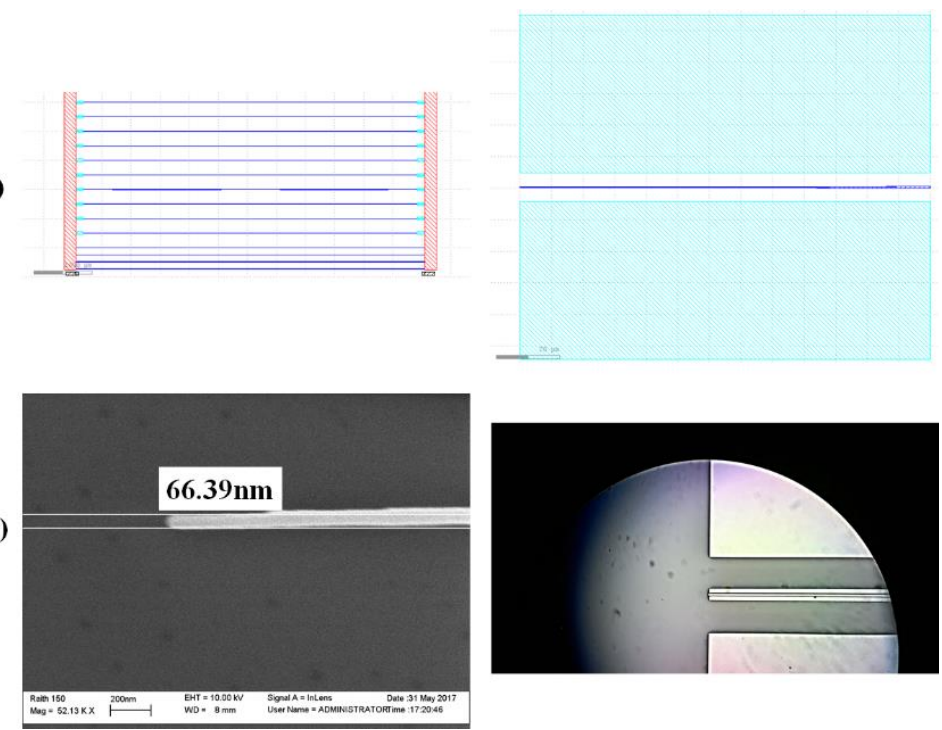

Figura 4.9: (a) GDS de la estructura para acoplamiento horizontal por medio de un convertidor de tamaño de modo, y (b) imagen SEM y del microscopio óptico del acoplador fabricado. 
La caracterización se realizó mediante la utilización del montaje para acoplo horizontal. De manera inicial se realizó un alineamiento fibra-objetivo (figura 4.10(a)), con el cual la fibra de entrada es alineada a la estructura de acoplo de la muestra. Una vez alineada la fibra, el haz de luz del modo acoplado a la guía en la interfaz del puerto de salida es visible en la cámara de infrarrojo (figura 4.10(b)) y el 50\% de la potencia óptica acoplada es captada por el fotodetector, pudiendo ser medida en el medidor óptico.
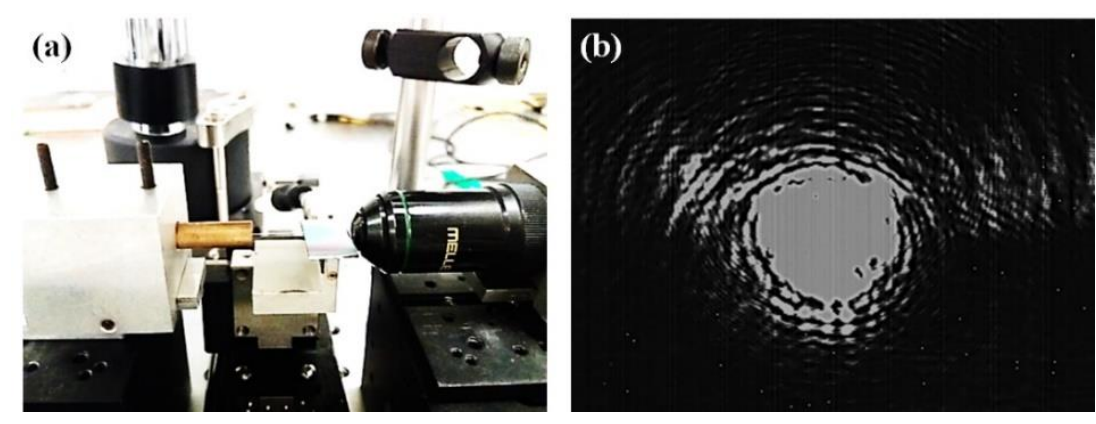

Figura 4.10: (a) Montaje de acoplamiento horizontal para alineamiento fibra-objetivo, y (b) punto de luz obtenido en la interfaz del puerto de salida de la muestra.

Después del alineamiento fibra-objetivo, se alineó una fibra al puerto de salida de la muestra (figura 4.11(a)). De esta manera, realizando un balance de potencias (Eq.4.2), teniendo en cuenta unas pérdidas de propagación en la guía de $\alpha_{w g} @ 1310 \mathrm{~nm}=5 \frac{\mathrm{dB}}{\mathrm{cm}} \mathrm{y}$ una longitud de guía de $L_{w g}=0.5 \mathrm{~cm}$, se estimaron las pérdidas de acoplo en $\alpha_{S S C} @ 1310 \mathrm{~nm}=4 \mathrm{~dB}$. El espectro normalizado de las pérdidas del acoplo obtenido tanto en caracterización como mediante simulación 3D-BPM se muestra en la figura 4.11(b).

$$
\alpha_{S S C}[d B]=\frac{1}{2}\left(P_{\text {in }}[d B m]-P_{o u t}[d B m]-\alpha_{w g} L_{w g}[d B]\right)
$$

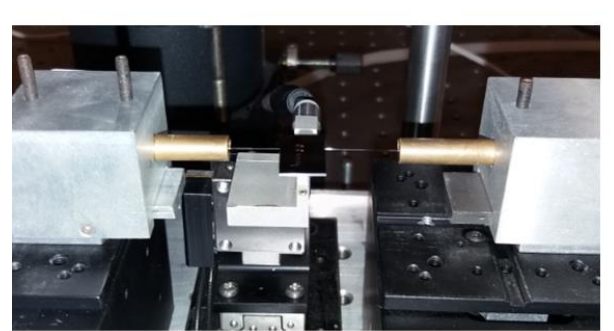

(a)

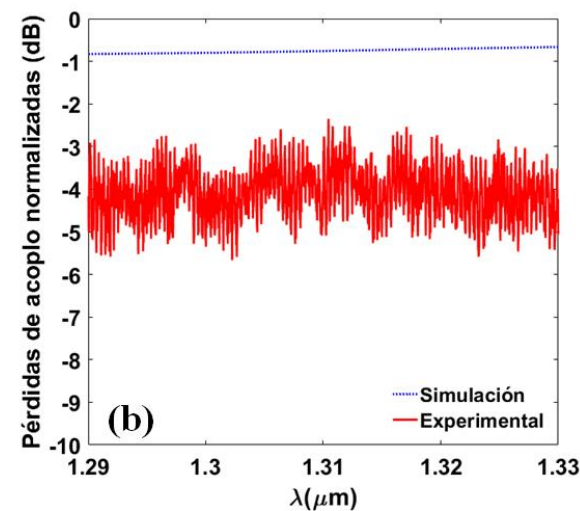

Figura 4.11: (a) Montaje de acoplamiento horizontal para alineamiento fibra a fibra, y (b) espectro de pérdidas de acoplo experimental y simulado.

Finalmente, se realizó una medida más precisa en el banco de alineamiento utilizando una estructura con varias fibras (fiber-array) ensamblada en los puertos de entrada y salida de la muestra (figura 4.12(a)). De esta manera, se obtuvieron unas pérdidas de acoplo de alrededor de 4dB. Sin embargo, realizando una adaptación de índices (index matching) 
Capítulo 4: Fabricación y resultados experimentales del esquema propuesto de integración híbrida de láser con cavidad externa

en la interfaz entre el chip de silicio y la fibra óptica, mediante un depósito de epoxi y curado ultravioleta (UV), las pérdidas de acoplo se redujeron a $3 \mathrm{~dB}$.

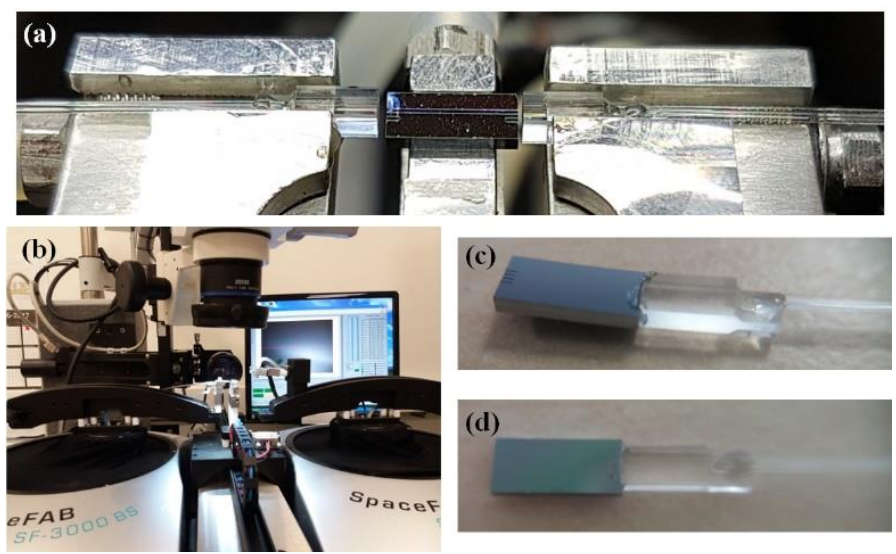

Figura 4.12: (a) Montaje de acoplamiento horizontal para alineamiento entre la fibra y la muestra, (b) banco de alineamiento, y (c), (d) muestra ensamblada a la fibra.

\subsubsection{Caracterización del filtro óptico basado en anillo}

El filtro óptico basado en anillo con FSR de 10nm y $12 \mathrm{~nm}$ fue también fabricado y caracterizado. En la figura 4.13(a) se detalla el GDS del anillo y la estructura fabricada. Se realizaron varias fotos SEM para corroborar los parámetros del anillo en especial de la separación entre la guía y el anillo (figura 4.13(b)).

(a)
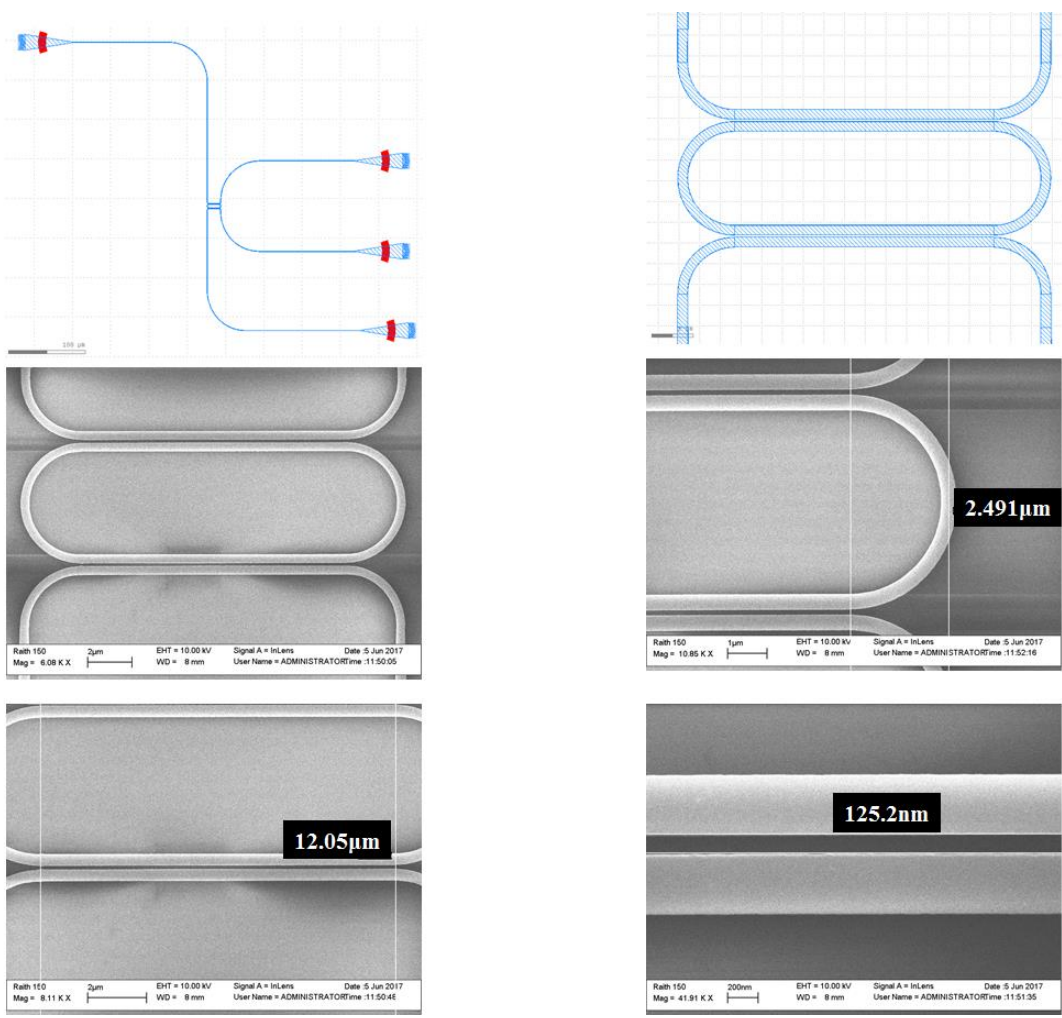

Figura 4.13: (a) GDS de la estructura de filtro basado en anillo resonante. (b) Imágenes SEM de la estructura fabricada. 
Las estructuras se caracterizaron haciendo uso del montaje de acoplo vertical y por tanto usando acopladores de red de difracción a la entrada y salida. En la figura 4.14 se puede observar el espectro normalizado obtenido tanto en caracterización como mediante simulación (Matlab) de los filtros con distinto FSR. Se observa que la resonancia se encuentra centrada alrededor de $\lambda=1305 \mathrm{~nm}$ y por tanto desplazada $5 \mathrm{~nm}$ respecto a la longitud de onda de diseño. Las pérdidas de inserción en el puerto de salida "drop" son de alrededor de $2 \mathrm{~dB}$ y $3 \mathrm{~dB}$ para un FSR de $10 \mathrm{~nm}$ y $12 \mathrm{~nm}$ respectivamente.
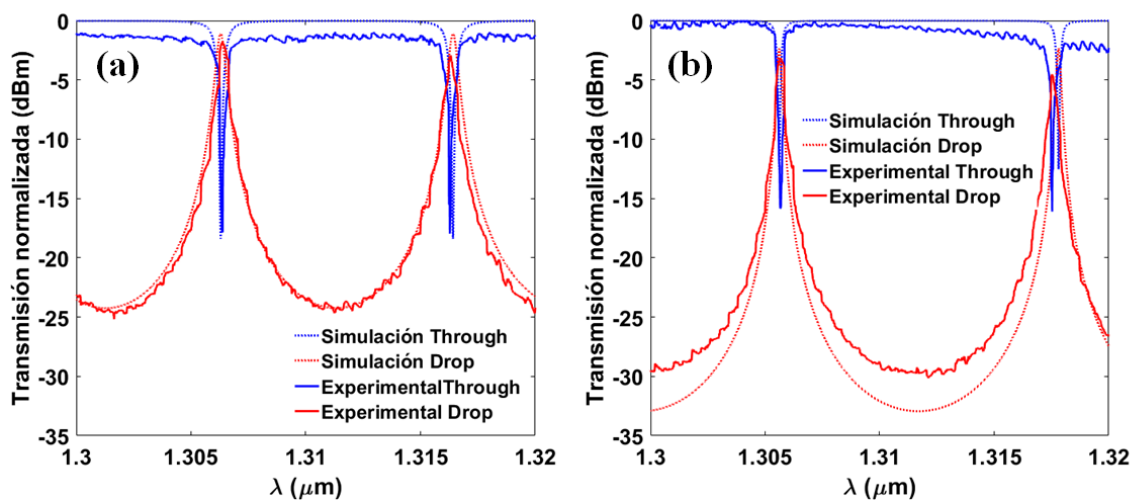

Figura 4.14: Espectro de transmisión del filtro de anillo resonante de FSR (a) 10nm y (b) $12 \mathrm{~nm}$.

Con los datos experimentales se estimó el factor de calidad, $Q$, y el ancho de banda a $3 \mathrm{~dB}, B W_{3 d B}$, de la resonancia en el puerto de salida "drop" para ambos diseños. Para el filtro de anillo con FSR de 10nm, el factor de calidad obtenido es de $Q=3266$ con un ancho de banda en la resonancia de $B W_{3 d B}=0.4 \mathrm{~nm}$. Mientras que para el filtro con FSR de $12 \mathrm{~nm}$, el factor de calidad obtenido es de $Q=4352$ y el ancho de banda en la resonancia de $B W_{3 d B}=0.3 \mathrm{~nm}$. Se observa que los valores experimentales son acordes a los obtenidos mediante simulación y por tanto con el diseño realizado.

\subsubsection{Caracterización del reflector}

Para la caracterización del reflector se utilizó una cavidad Fabry-Perot (FP) (figura 4.15) debido a que la medida directa de la estructura no resultó precisa. Mediante el uso de la cavidad FP se estimó la reflectividad del DBR mediante la respuesta en transmisión

$$
\frac{P_{\max }}{P_{\min }}=\frac{1+R e^{-\alpha L}}{1-R e^{-\alpha L}}
$$

siendo $P_{\max }$ y $P_{\min }$ la potencia máxima y mínima en cada resonancia, $R$ la reflectividad y $\alpha$ las pérdidas de propagación en la guía de la cavidad.

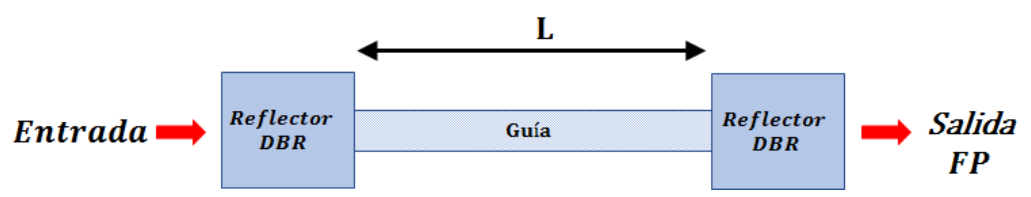

Figura 4.15: Cavidad Fabry-Perot utilizada para la caracterización del reflector. 
Capítulo 4: Fabricación y resultados experimentales del esquema propuesto de integración híbrida de láser con cavidad externa

La caracterización se llevó a cabo mediante el montaje de acoplo vertical. El GDS de la estructura diseñada con las cavidades FP se muestra en la figura 4.16(a). Una vez fabricada la muestra, se realizaron varias imágenes SEM para comprobar los parámetros de diseño (figura 4.16(b)).

(a)

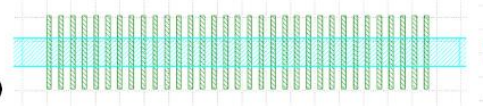

(b)

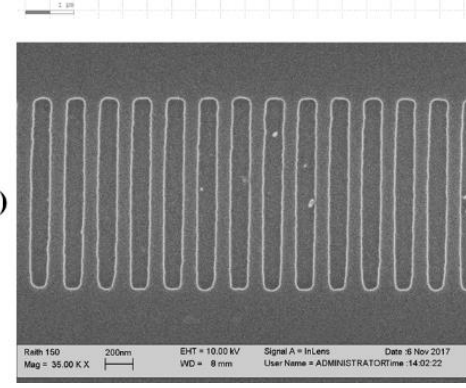

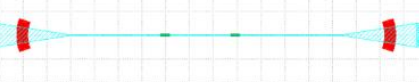

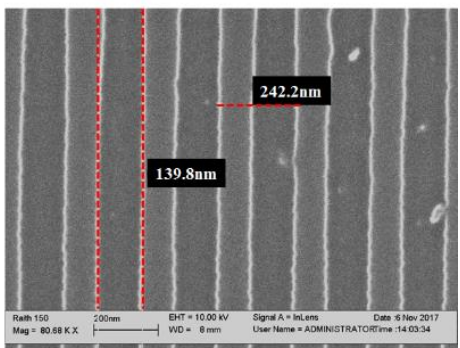

Figura 4.16: (a) GDS de la estructura del reflector. (b) Imágenes SEM del reflector fabricado.

En la figura 4.17(a, b) se puede observar el espectro de transmisión experimental de la cavidad FP con un DBR de reflectividad en diseño de $R=50 \%$ y $R=95 \%$ respectivamente. Se observa también el espectro de transmisión de una guía sin reflectores utilizado para normalización, correspondiente al espectro de la red de difracción. En la figura 4.17(c, d) se muestra el espectro de transmisión normalizado de la cavidad FP obtenido tanto en caracterización como mediante simulación Matlab para cada reflectividad.
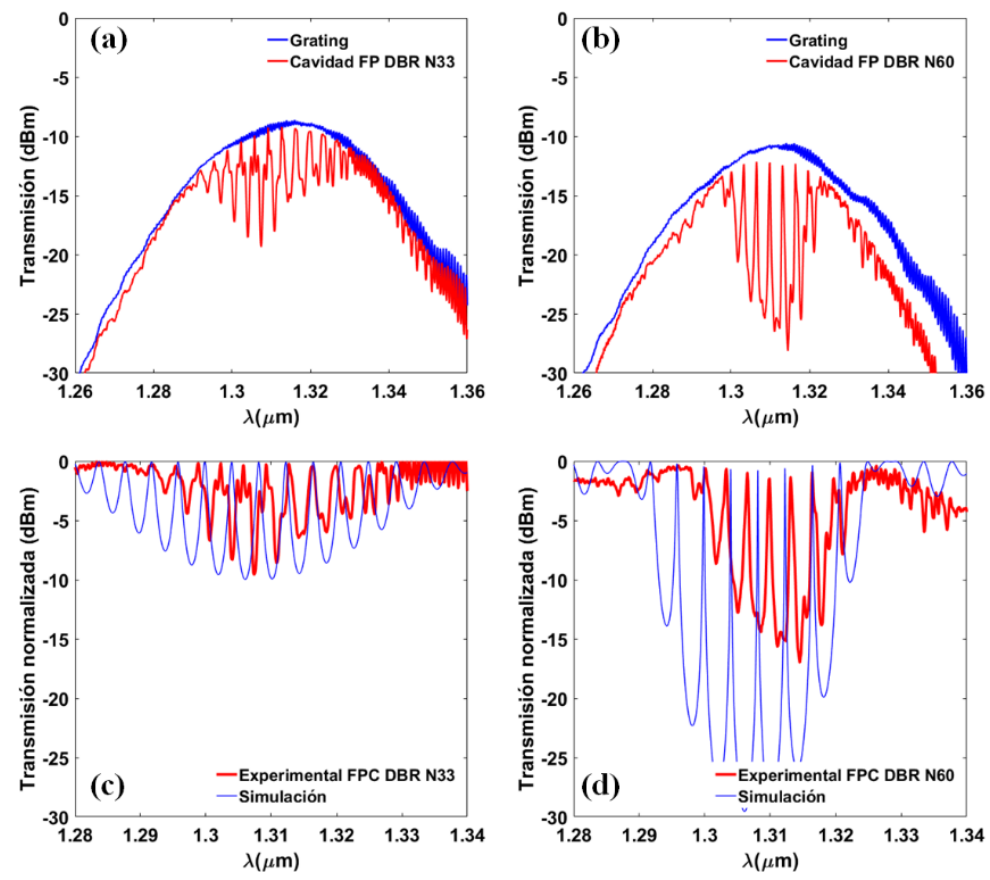

Figura 4.17: (a, b) Espectro de transmisión experimental. (c, d) Espectro normalizado de la cavidad Fabry-Perot formada por un DBR con 33 periodos $(R=50 \%)$ y 60 periodos $(R=95 \%)$ respectivamente 
La longitud de la cavidad FP implementada es de $L=50 \mu m$, de manera que el espaciado de los modos de cavidad es de $4 \mathrm{~nm}$, valor que coincide con el espaciado entre resonancias observado en los resultados experimentales. Además, se observa que la respuesta de la cavidad FP obtenida en transmisión se encuentra centrada alrededor de la longitud de onda de diseño. Utilizando la Eq. 4.3 se verifica que la reflectividad alcanzada de manera experimental en el reflector con 33 periodos es del $49 \%$, mientras que para el reflector con 60 periodos la reflectividad alcanzada es del $73 \%$.

\subsection{Desarrollo y caracterización del láser con cavidad externa}

En una primera instancia se realizó una medición del perfil del haz de luz emitido por el chip de ganancia QD en campo cercano. Para ello se usó un equipo de visualización SNOM (Nanonics Imaging Multiview 4000), tal como se puede ver en la figura 4.18. El chip-QD se montó sobre un soporte para conseguir emisión vertical y así poder realizar la medida.
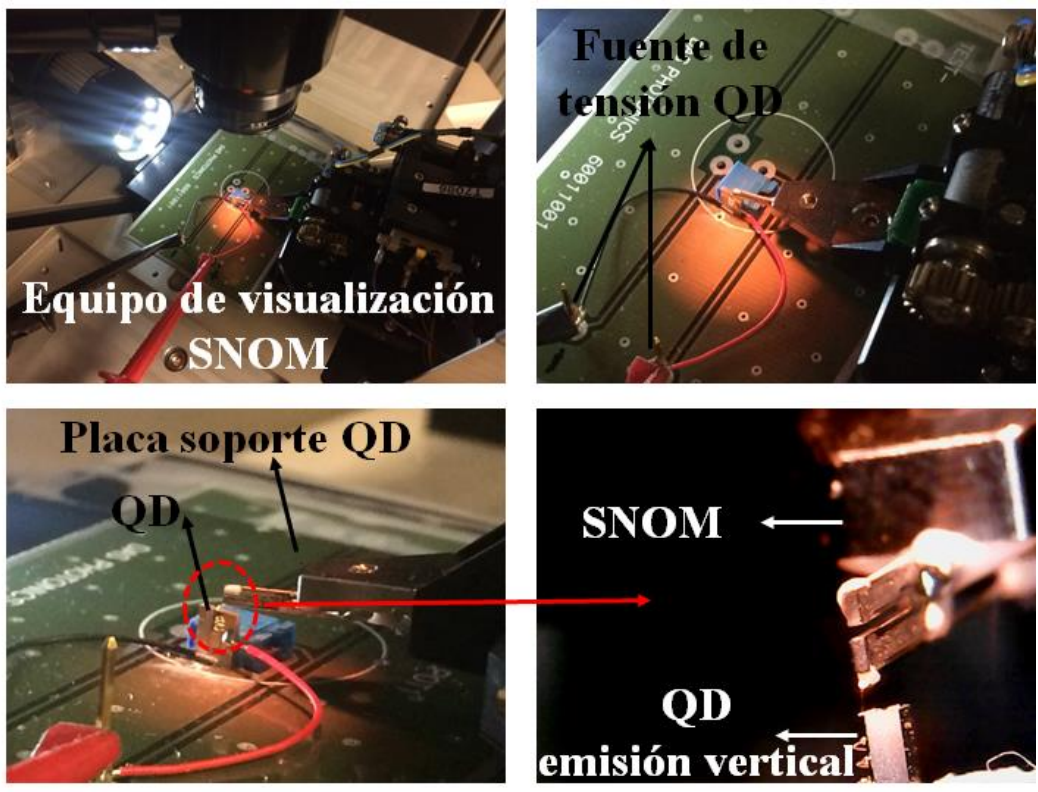

Figura 4.18: Fuente de luz QD preparada para emisión vertical y detalles de la medida realizada con el equipo SNOM.

De esta manera se corroboraron de manera experimental las dimensiones del modo de propagación del QD, modeladas en Matlab de acuerdo con las especificaciones del fabricante respecto a los ángulos de divergencia en campo lejano y con las cuales se obtuvo (capítulo 3) las dimensiones del diámetro del modo de propagación en el campo cercano. El perfil del campo cercano medido se muestra en la figura 4.19 mientras que las dimensiones experimentales y analíticas del modo se muestran en la tabla 4.1. Ambos valores son similares, siendo una buena indicación de que el diseñó del convertidor de tamaño de modos para acoplo entre el chip de ganancia QD y una guía SOI se realizó correctamente. 
Capítulo 4: Fabricación y resultados experimentales del esquema propuesto de integración híbrida de láser con cavidad externa

\begin{tabular}{|c|c|c|}
\hline $\begin{array}{l}\text { Dimensiones del } \\
\text { modo de } \\
\text { propagación en } \\
\text { campo cercano }\end{array}$ & Analítico & Experimental \\
\hline Slow axis (x) & $5.623 \mu \mathrm{m}$ & $5.112 \mu \mathrm{m}$ \\
\hline Fast axis (y) & $0.922 \mu \mathrm{m}$ & $0.972 \mathrm{~nm}$ \\
\hline
\end{tabular}

Tabla 4.1: Diámetro del modo de propagación de la fuente de luz QD en campo cercano tanto para el eje lento y rápido.

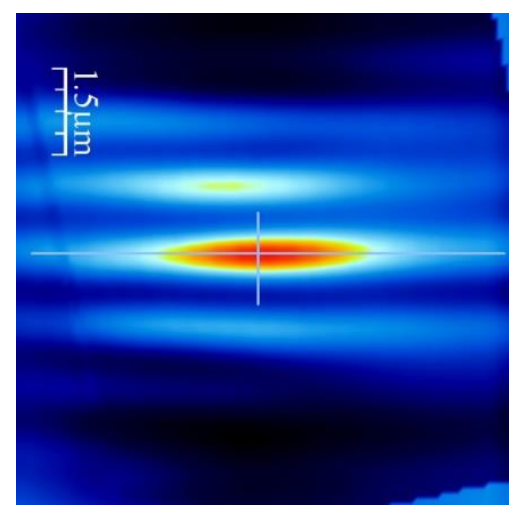

Figura 4.19: Perfil del campo cercano obtenido con el equipo SNOM del chip-QD.

El siguiente paso fue la caracterización de la fuente de luz QD. Para esto se alineó al objetivo y de éste directamente al fotodetector (figura 4.20(a, b, c)). Mediante la utilización de una fuente de voltaje DC, se aplicó una variación de tensión al QD. De esta manera, se caracterizó el consumo de corriente y la potencia de salida del láser (figura 4.20(d)).
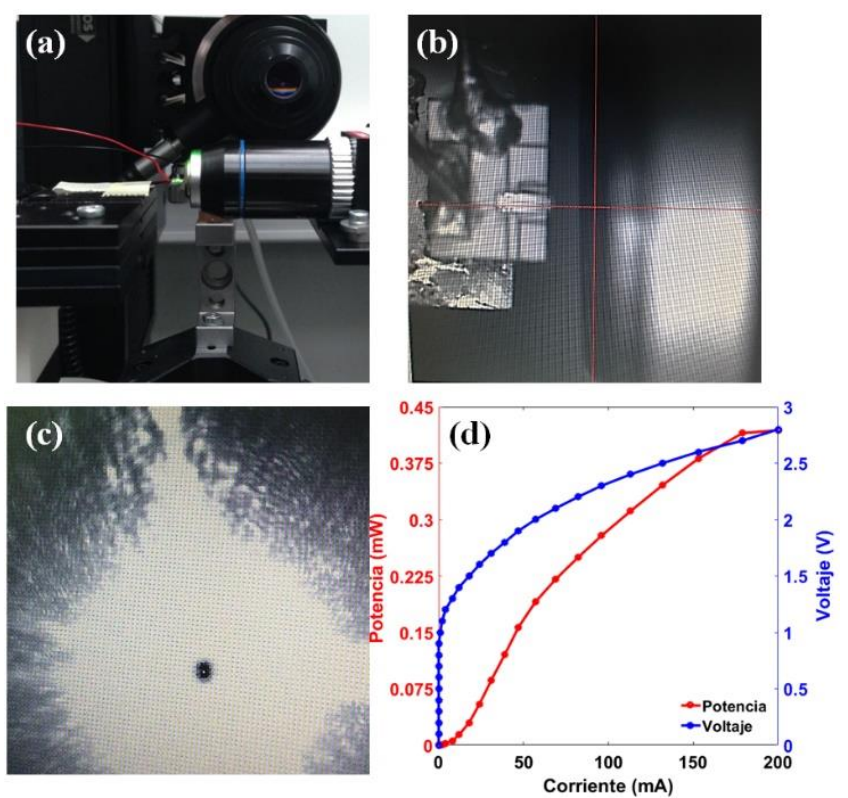

Figura 4.20: (a, b, c) Caracterización del chip de ganancia QD alineado respecto a objetivo para la obtención de la (d) potencia de salida en función de la corriente suministrada.

Finalmente, se obtuvo el espectro de emisión del QD. Para ello, el QD se alineó a una fibra óptica conectada a un analizador de espectros óptico OSA (Ando AQ6317C) (figura 4.21(a, b)). El espectro de emisión obtenido corresponde a un suministro de corriente $I=$ 
$200 m A$ proporcionado por la fuente de voltaje DC conectada al QD (figura 4.21(c)). Se observa que la respuesta coincide en gran medida con la proporcionada por el fabricante del QD (figura 4.22).
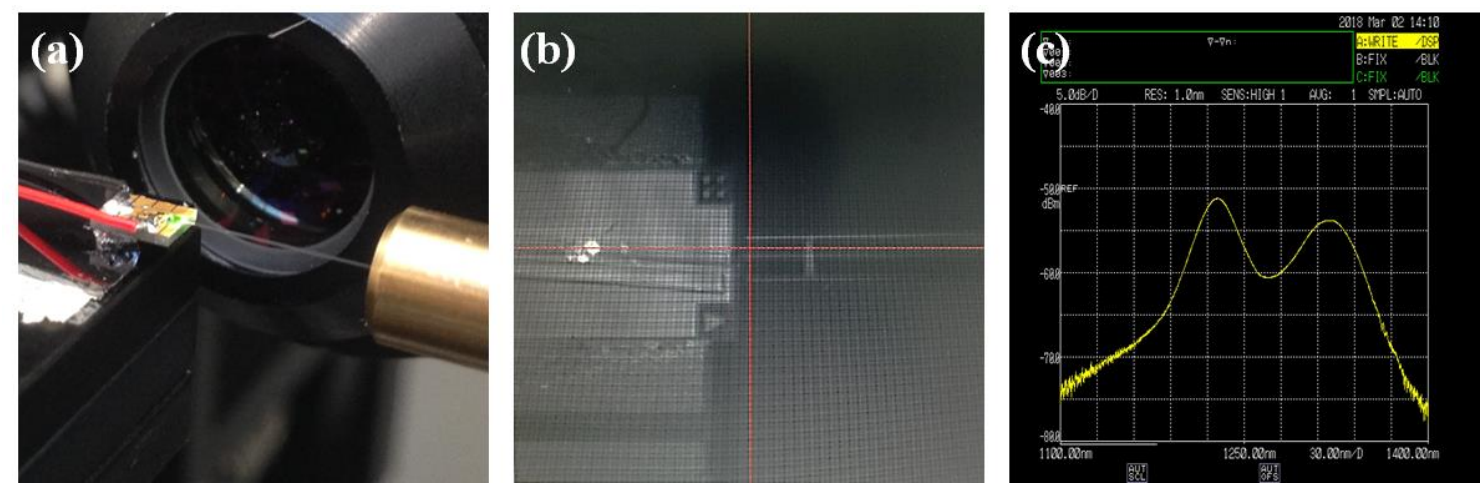

Figura 4.21: Espectro de emisión del chip de ganancia QD obtenido mediante el alineamiento a una fibra óptica y a una corriente de suministro en el QD de $I=200 \mathrm{~mA}$.

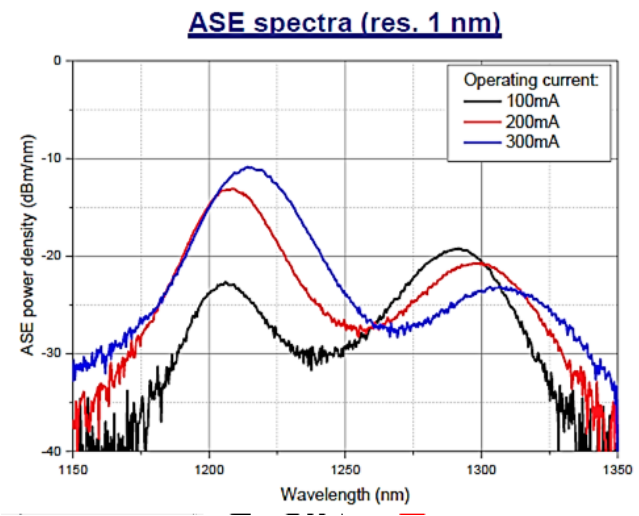

Figura 4.22: Especificaciones del chip de ganancia QD respecto al espectro de ganancia en función de la corriente de suministro.

\subsubsection{Caracterización del QD y acoplador de tamaño de modos}

La caracterización del convertidor de tamaño de modo para acoplo entre el chip de ganancia QD y el chip de silicio se llevó a cabo mediante el banco de alineamiento y acoplo horizontal. El GDS de la estructura de acoplo y de la muestra se observan en la figura 4.23(a), la cual se giró $11.54^{\circ}$ respecto al extremo del taper invertido. De esta manera, se alcanzó un correcto alineamiento al haz de luz de emitido por el QD. Para la realización de la medida se utilizó una muestra con varias estructuras. El QD es alineado al puerto de entrada, con el acoplador diseñado a una guía de anchura 500nm y de longitud $L_{w g}=0.5 \mathrm{~cm}$, que conecta a un puerto de salida provisto por otro acoplador para acoplamiento a fibra óptica, tal como se muestra en la figura 4.23(b). 
Capítulo 4: Fabricación y resultados experimentales del esquema propuesto de integración híbrida de láser con cavidad externa

(a)
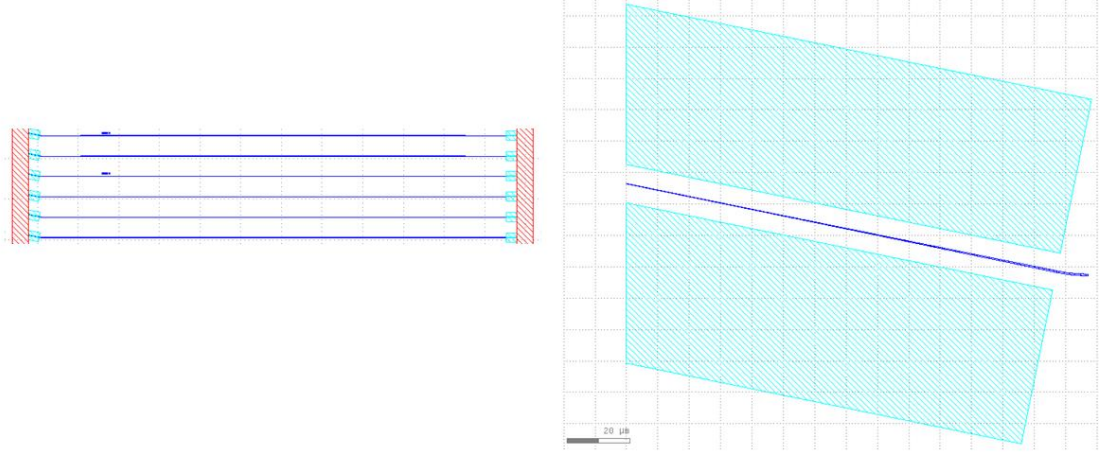

(b)
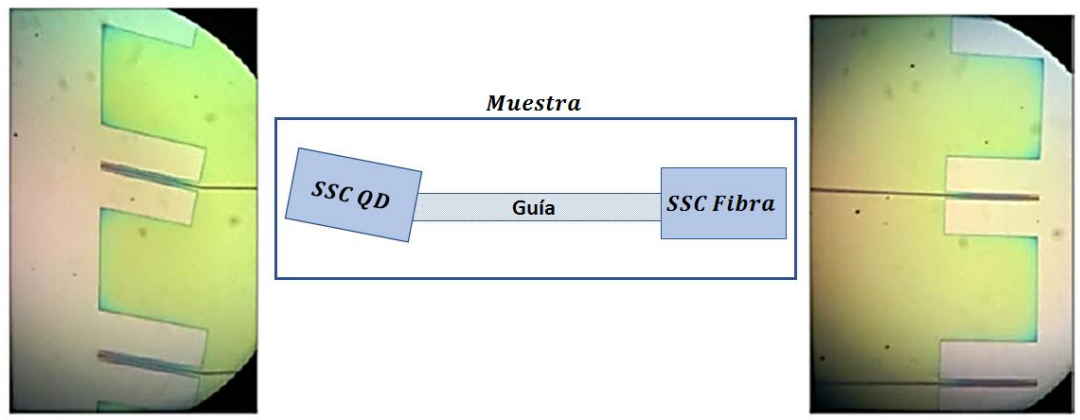

Figura 4.23: (a) GDS de la estructura para acoplamiento entre el chip QD y el chip de silicio, y (b) detalle de la estructura utilizada e imágenes de microscopio óptico de los acopladores fabricados.

Inicialmente se realizó un alineamiento activo entre el QD, la muestra y el objetivo en el puerto de salida (figura 4.24(a, b)). Así, el punto de luz a la salida del acoplador a fibra se visualizó en la cámara una vez alineado (figura 4.24(c)). De esta manera, queda demostrado el acoplo del QD al chip de silicio.
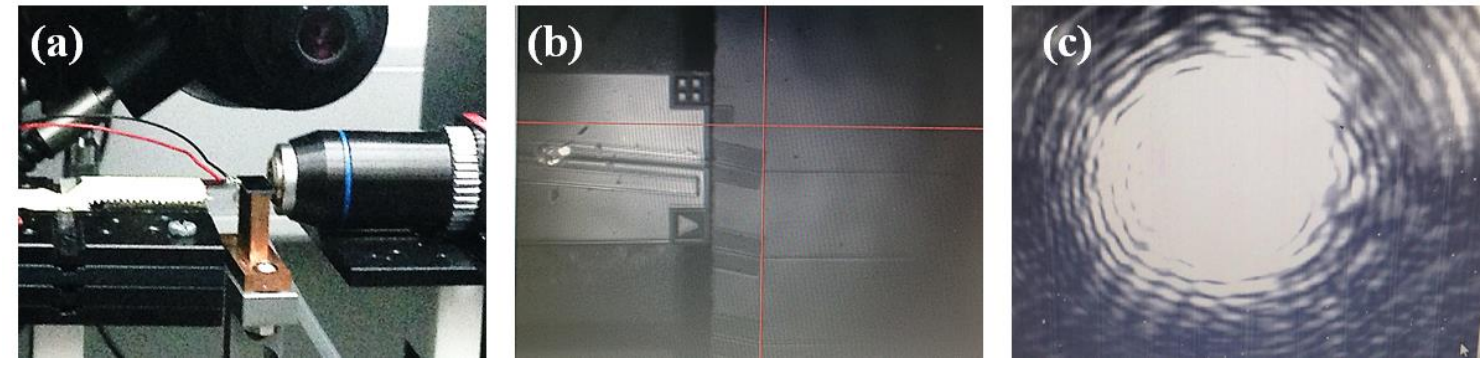

Figura 4.24: (a) Alineamiento activo del chip de ganancia QD respecto a objetivo. (b) Alineamiento del QD al puerto de entrada de la muestra. (c) Punto de luz visualizado en la cámara de infrarrojo después del alineamiento.

Una vez alineado el QD, se quitó el objetivo y se reemplazó por una fibra óptica (figura 4.25(a)). De esta manera se realizó un alineamiento activo de la fibra respecto al puerto de salida (figura 4.25(b)), midiendo una potencia de salida de $P_{\text {out }}=-16 \mathrm{dBm}$ para una tensión aplicada sobre el QD de 2.7v y un suministro de corriente de $200 \mathrm{~mA}$ (figura $4.25(\mathrm{c})$ ). Teniendo en cuenta unas pérdidas de $2.5 \mathrm{~dB}$ en la guía (pérdidas de propagación $\alpha_{w g}=5 \frac{d B}{c m}$ y longitud de la guía $L_{w g}=0.5 \mathrm{~cm}$ ), unas pérdidas en el acoplador a fibra de alrededor de $\alpha_{S S C_{-} \text {fiber }} \approx 4 d B$ y una potencia en el puerto de entrada de $P_{\text {in }}=-4 d B m$ 
(figura 4.20(d)) se estimaron las pérdidas del acoplador a QD en $\alpha_{S S C_{-} Q D} \approx 5.5 d B$ a partir del balance de potencias de

$$
\alpha_{S S C_{-} Q D}[d B]=P_{\text {in }}[d B m]-P_{\text {out }}[d B m]-\alpha_{w g} L_{w g}[d B]-\alpha_{S S C_{f i b e r}}[d B]
$$

Finalmente, el espectro de emisión del QD acoplado a la guía fue obtenido conectando la fibra óptica de salida al analizador de espectros OSA, tal como se observa en la figura $4.25(\mathrm{~d})$.
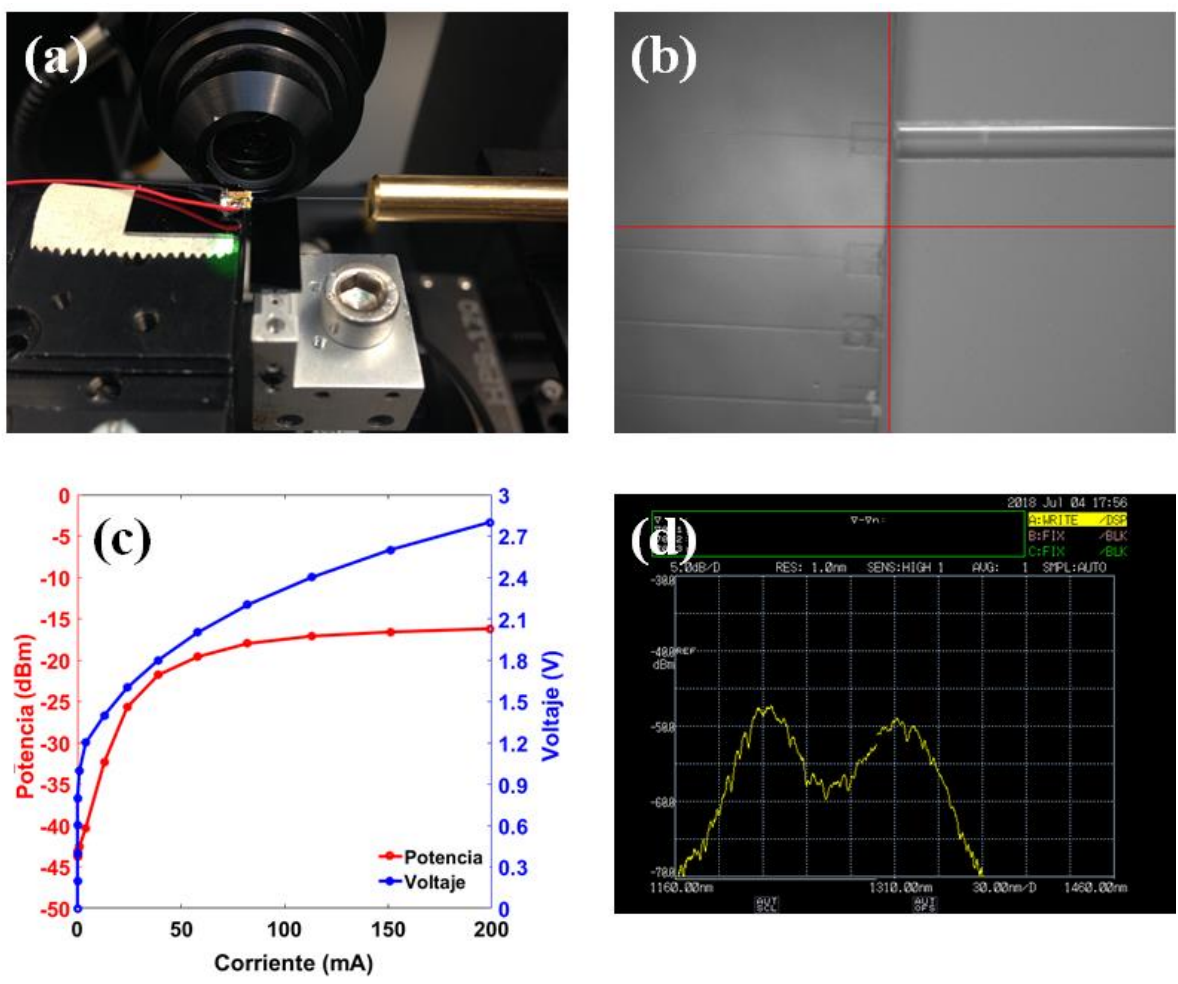

Figura 4.25: (a) Alineamiento activo del chip de ganancia QD respecto a una fibra óptica. (b) Alineamiento de la fibra óptica en el puerto de salida de la muestra. (c) Potencia óptica acoplada a una guía detectada en el puerto de salida en función de la corriente suministrada al QD. (d) Espectro de emisión del QD acoplado a la guía.

\subsubsection{Caracterización de la cavidad externa}

Para la caracterización de la cavidad externa antes de la integración híbrida con el chip de ganancia QD, se formó una cavidad Fabry-Perot utilizando los DBR diseñados e incluyendo el filtro de anillo en el interior de la cavidad (figura 4.26). De esta manera, utilizando el montaje de acoplo vertical y empleando los acopladores a fibra diseñados, se alineó una fibra óptica en cada puerto y se midió la potencia de salida de la cavidad Fabry-Perot, con lo cual se evaluó el funcionamiento del filtro de anillo resonante y DBR. 
Capítulo 4: Fabricación y resultados experimentales del esquema propuesto de integración híbrida de láser con cavidad externa

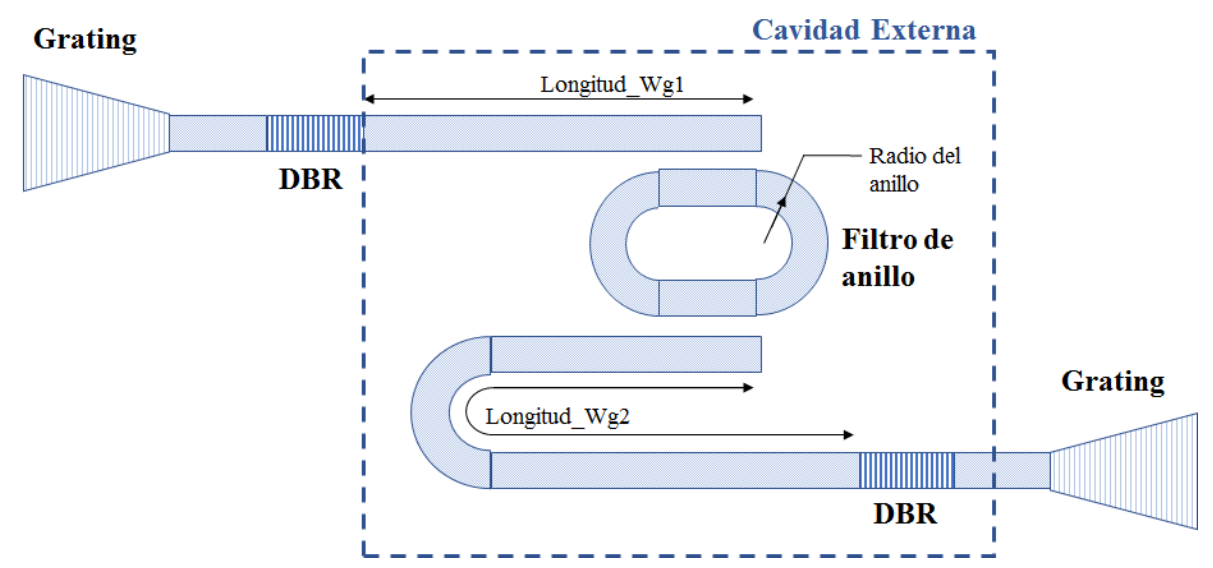

Figura 4.26: Esquemático de una cavidad Fabry-Perot formada por guías de onda que conectan a dos DBR de igual reflectividad y un filtro de anillo resonante entre ellos.

El GDS empleado para la caracterización de la cavidad externa se muestra en la figura 4.27. La reflectividad del DBR es de $R=95 \%$, y la longitud total de la cavidad FP es de $L_{C F P}=500 \mu m$, para la cual el FSR estimado de los modos resonantes en la cavidad es de alrededor de $0.4 \mathrm{~nm}$.

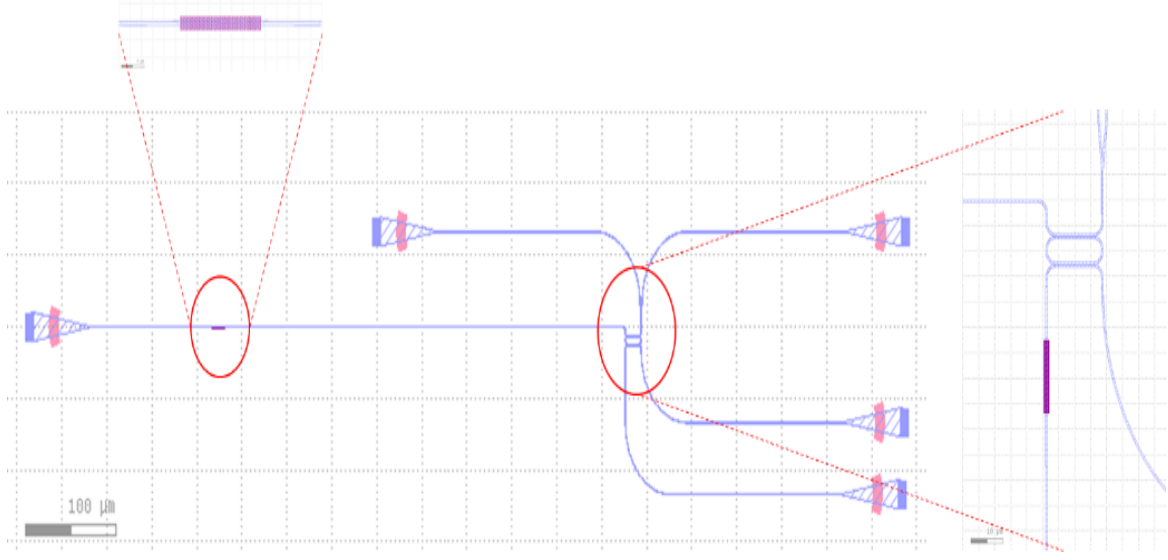

Figura 4.27: GDS utilizado para la caracterización de la cavidad externa.

En la figura 4.28 se muestra el espectro experimental de salida de la cavidad FP fabricada junto al espectro simulado en Matlab observándose una buena correspondencia en los resultados. El FSR experimental medido de los modos de cavidad es de alrededor de $0.3 \mathrm{~nm}$. 


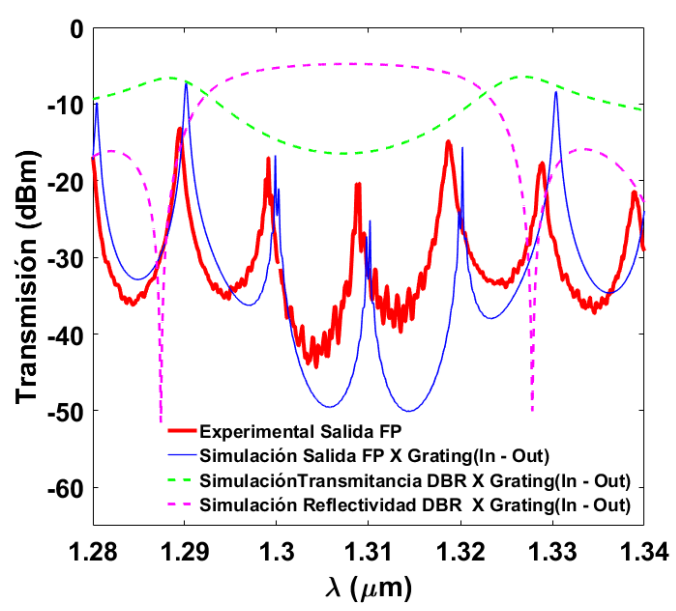

Figura 4.28: Resultados experimentales y de simulación de la cavidad FP con filtro de anillo.

\subsubsection{Sintonización termo-óptica}

El proceso de sintonización termo-óptica se caracterizó mediante la fabricación de microcalentadores de oro. De esta forma, una capa de oro de espesor 500nm fue depositada sobre la capa de óxido que recubre el anillo a una separación de 700nm. El diseño del GDS del calentador y filtro de anillo utilizado para la caracterización de la sintonización termo-óptica se muestra en la figura 4.29(a). Después de la fabricación y del proceso de metalización, se realizaron varias imágenes con el microscopio óptico (figura 4.29(b)). Para la caracterización se empleó el montaje de acoplo vertical. Además, se usó una fuente de voltaje y puntas de DC para aplicar una tensión sobre los electrodos. De esta manera se provocó un incremento de temperatura sobre la guía del anillo resonante, produciendo el efecto termo-óptico en el silicio.

(a)

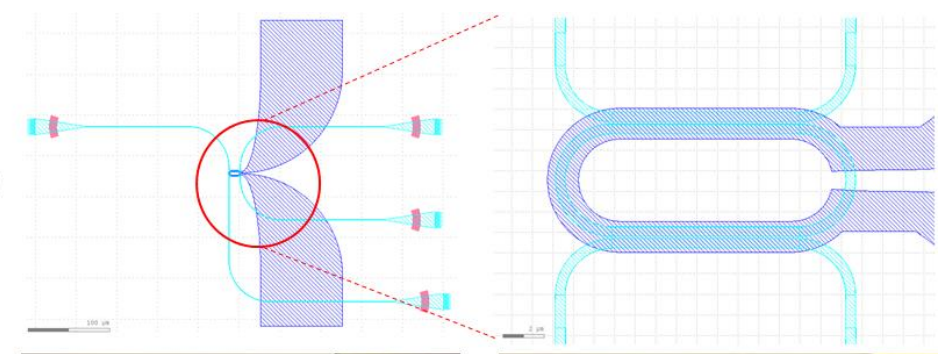

(b)
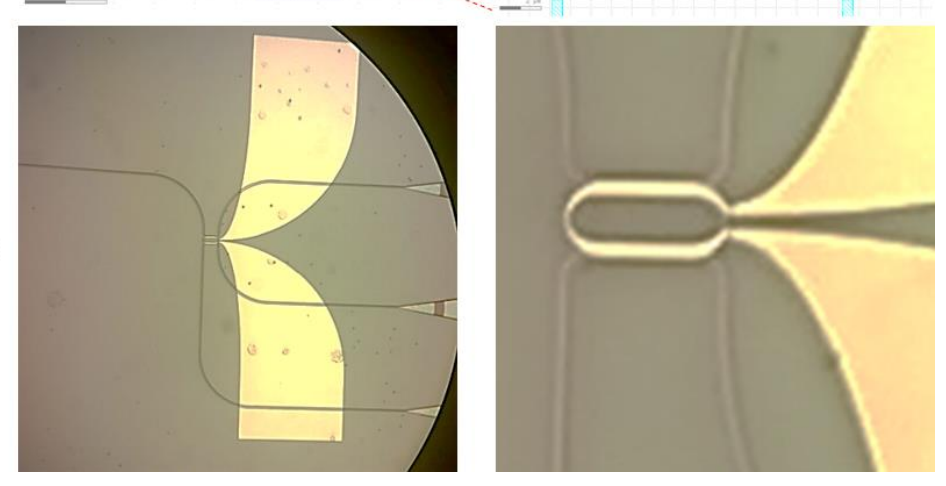

Figura 4.29: (a) GDS del filtro de anillo resonante y micro-calentadores para la sintonización termo-óptica. (b) Imágenes de microscopio óptico de la estructura fabricada. 


\section{Capítulo 4: Fabricación y resultados experimentales del esquema propuesto de integración híbrida de láser con cavidad externa}

En la figura 4.30(a) se muestra el procedimiento experimental para la sintonización del filtro de anillo resonante medido en el puerto de salida "through". Los resultados muestran un desplazamiento superior al FSR del anillo (figura 4.30(b)), correspondiente a un desfase de $2 \pi$ (figura $4.30(\mathrm{c})$ ), con un consumo de potencia de alrededor de $60 \mathrm{~mW}$.
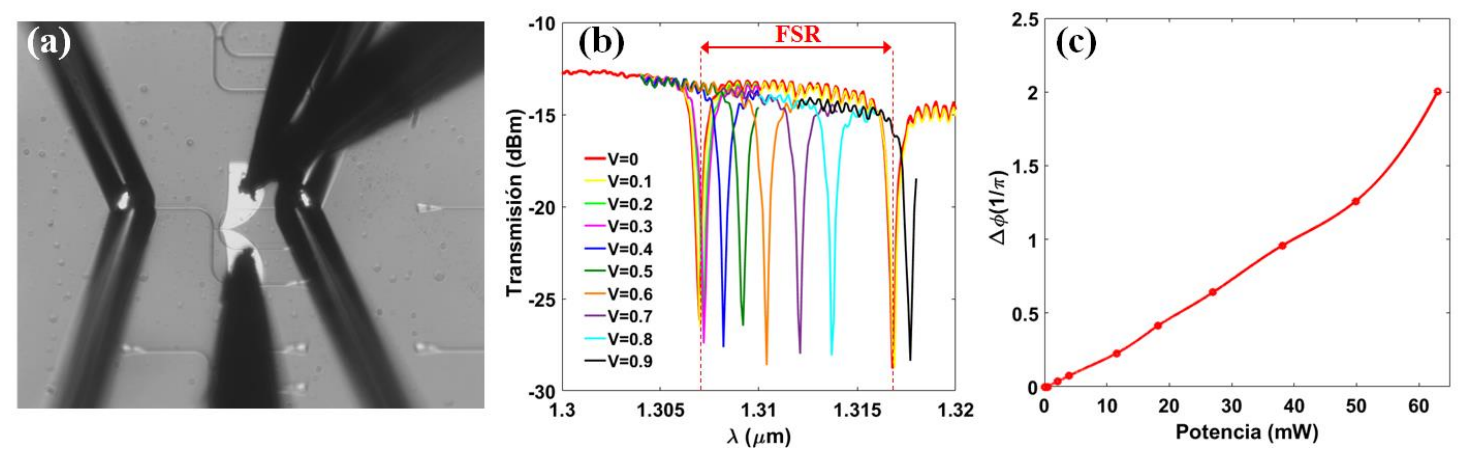

Figura 4.30: (a) Imagen durante la caracterización de la sintonización termo-óptica. (b) Desplazamiento de la resonancia del filtro de anillo debido al efecto termo-óptico para diferentes tensiones aplicadas, y (c) variación de la fase óptica en función del consumo de potencia en los calentadores.

Igual que la sintonización termo-óptica del anillo resonante, se realizaron otras pruebas de sintonización sobre una cavidad Fabry-Perot con el filtro de anillo. De esta manera, se colocaron calentadores sobre la estructura del anillo además de sobre una parte de la guía de la cavidad. De nuevo, por medio del efecto termo-óptico se sintonizó la respuesta de la cavidad FP. Por un lado, la sintonización del filtro de anillo resonante permitió seleccionar la longitud de onda de filtrado. Por otro lado, la variación de fase inducida sobre una parte de la guía de la cavidad permitió desplazar los modos de cavidad de tal manera que se alcanzó un alineamiento entre éstos y la resonancia del filtro de anillo maximizando la potencia de salida de la cavidad FP. En la figura 4.31 se puede observar el GDS de la estructura y de los calentadores diseñados para la sintonización termo-óptica tanto sobre el anillo resonante como sobre una parte de la guía de la cavidad.
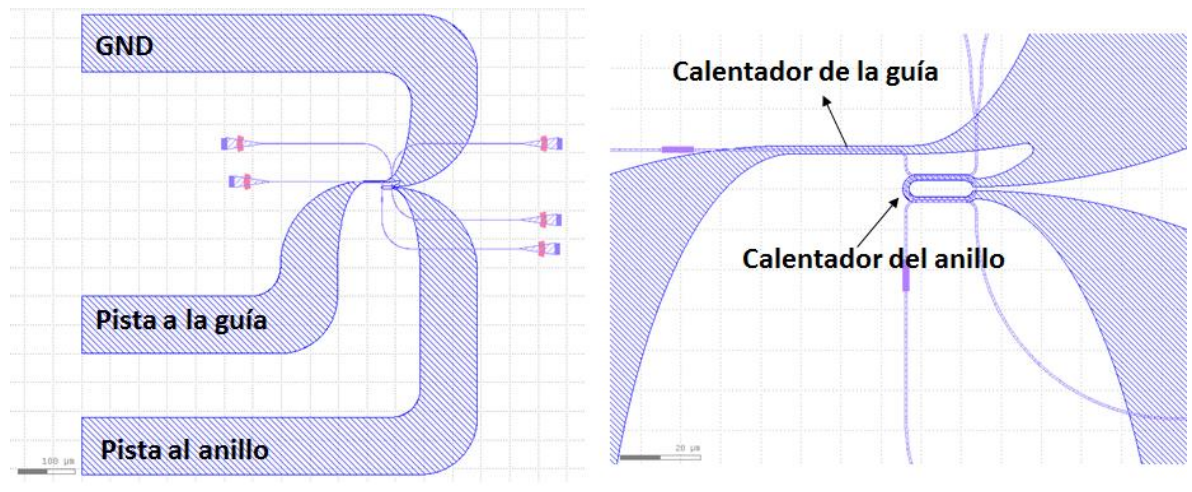

Figura 4.31: GDS de la cavidad Fabry-Perot utilizada para la sintonización del filtro de anillo y guía de la cavidad mediante el efecto termo-óptico.

La longitud de la cavidad es de alrededor de $L_{C F P}=500 \mu \mathrm{m}$ y la longitud del calentador sobre la guía es de $L_{H W G}=200 \mu m$. Se empleó el montaje de acoplo vertical para la 
caracterización de cada uno de los calentadores. En la figura 4.32(a) se muestra una imagen de la caracterización del calentador sobre la guía de la cavidad. El FSR de los modos de cavidad medidos es de $0.3 \mathrm{~nm}$. En las figuras 4.32 (b) se observan las medidas experimentales de la caracterización del calentador sobre la guía usando reflectores con $R=95 \%$. Se constató que el cambio de fase inducido sobre la guía mediante el efecto termo-óptico permite cambiar la posición de los modos de cavidad y alinearlos a la resonancia del anillo, maximizando la potencia de salida. Por otro lado, en las figuras 4.32(c) se muestra la sintonización de la respuesta de la cavidad FP mediante el desplazamiento del espectro del filtro de anillo usando en este caso reflectores con $R=$ $50 \%$. Finalmente, en la figura $4.32(d)$ se muestra el consumo de potencia requerido para inducir un desfase de fase de $2 \pi$ tanto para el calentador del filtro de anillo resonante y de la guía siendo de alrededor de $45 \mathrm{~mW}$ y $20 \mathrm{~mW}$ respectivamente.
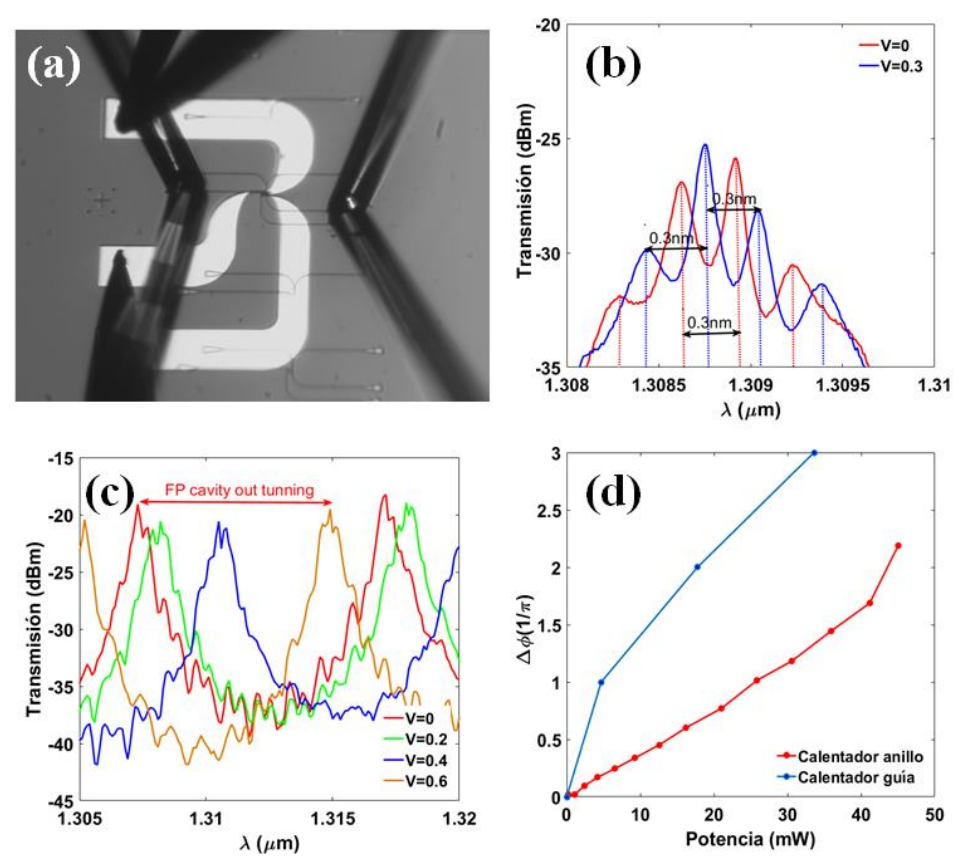

Figura 4.32: (a) Imagen durante la caracterización de la sintonización termo-óptica del calentador sobre la guía. (b) Caracterización del calentador sobre guía y desplazamiento de los modos longitudinales por medio del efecto termo-óptico en la salida de la cavidad FP. (c) Caracterización del filtro de anillo y sintonización termo-óptica de la salida de la cavidad FP. (d) Consumo de potencia requerida para un desfase de $2 \pi$ para el calentador en la guía y en el filtro de anillo resonante.

\subsubsection{Integración de fotodetector}

La posibilidad de disponer un puerto de monitorización en el filtro de anillo permite evaluar su funcionamiento tras la integración híbrida del láser de cavidad externa. Por ello, se desarrolló también el proceso de integración de un fotodetector en el chip de silicio mediante la técnica de "flip-chip bonding" y sobre un acoplador de red de difracción a la salida del puerto "through" del anillo resonante. Una vez integrado el fotodetector, correspondiente a un diodo PIN de una aleación de InGaAs/InP, la potencia óptica proveniente de la red de difracción puede ser detectada en la cara frontal del 
Capítulo 4: Fabricación y resultados experimentales del esquema propuesto de integración híbrida de láser con cavidad externa

fotodetector. Para que el fotodetector funcione se debe aplicar una tensión en polarización inversa a los contactos del ánodo y cátodo. Para ello se integraron pistas metálicas de oro sobre el puerto "through" del filtro de anillo. En la figura 4.33(a) se muestra una imagen del fotodetector visto en un microscopio óptico. Se realizó el diseño de la máscara del fotodetector de acuerdo a las especificaciones del fabricante y de los electrodos (figura 4.33(b)). Por último, las pistas de oro de espesor 500nm fueron depositadas sobre la capa de óxido que cubre la muestra (figura 4.33(c)).
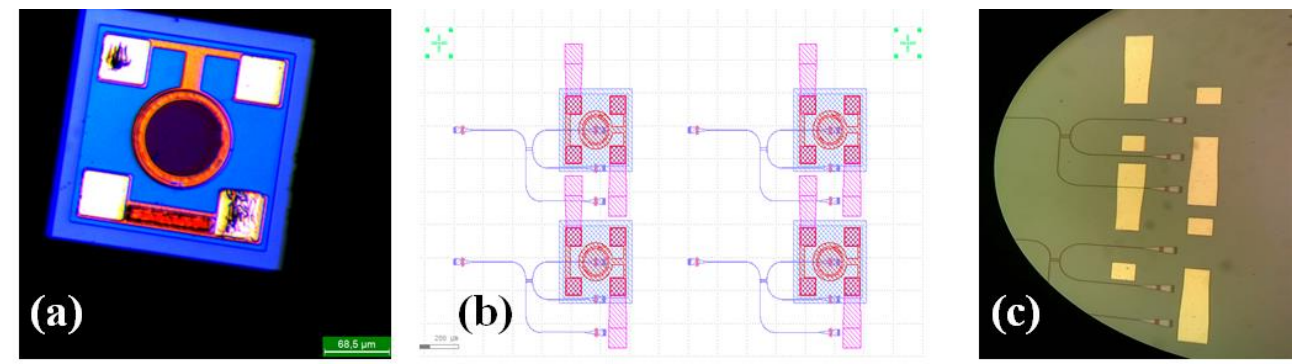

Figura 4.33: (a) Imagen frontal del fotodetector vista en el microscopio óptico. (b) GDS con la máscara del fotodetector y electrodos. (c) Imagen de la muestra en el microscopio óptico con las pistas de oro.

Por medio de la técnica "flip-chip", se colocó el fotodetector boca abajo sobre el acoplador de red de difracción. Para las conexiones entre el fotodetector y las pistas de oro se utilizaron bolas de soldadura de un material conductor eléctrico, que fueron depositadas por medio de un sistema de soldadura láser (figura 4.34(a)). Posteriormente a la colocación de las bolas de soldadura, se colocó el fotodetector sobre la red de difracción y se alineó a las pistas de oro con la utilización de un equipamiento automatizado (figura 4.34(b)). Finalmente, se soldó el fotodetector a las pistas de oro con las bolas de soldadura mediante un proceso de termocompresión (figura 4.34(c)).
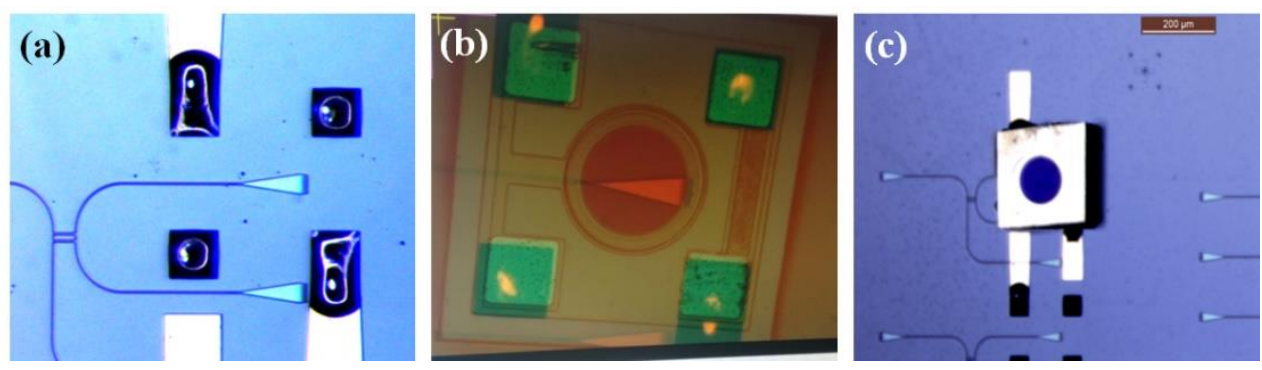

Figura 4.34: (a) Bolas de soldadura después de haber sido colocadas. (b) Imagen del proceso de alineamiento entre las pistas de la muestra y los electrodos del fotodetector. (c) Imagen de la integración del fotodetector después del proceso de "flip-chip bonding".

Después del proceso "flip-chip" se realizó la caracterización del chip de silicio con el fotodetector integrado empleando el montaje de acoplo vertical. Mediante puntas de DC se aplicó una tensión sobre el fotodetector en polarización inversa y se midió la respuesta eléctrica (figura 4.35(a, b)). En la figura 4.35(c) se muestra el espectro de transmisión medido en el puerto "Through" del anillo resonante correspondiente a la luz detectada en el fotodetector (corriente en función de longitud de onda). Como se puede observar existe 
una buena correspondencia con el espectro de potencia óptica de transmisión previamente medido (figura 4.35(d)), demostrando así el proceso de integración.
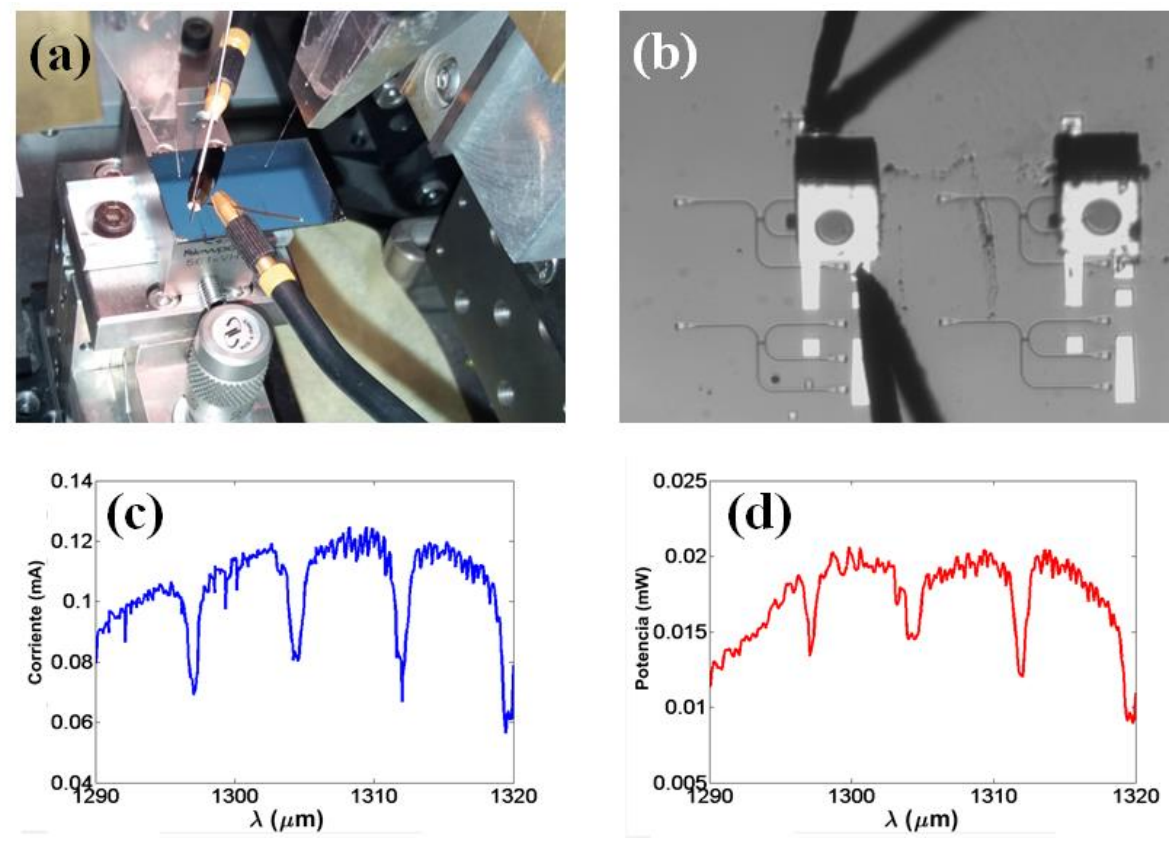

Figura 4.35: (a) Imagen durante la caracterización del chip de silicio con el fotodetector integrado. (b) Imagen de las puntas de DC sobre los electrodos para medir la respuesta eléctrica. (c) Corriente de salida medida en el fotodetector, y (d) potencia óptica medida en el anillo resonante antes de la integración del fotodetector.

\subsubsection{Demostración del funcionamiento del láser}

Una vez todos los bloques de diseño fueron caracterizados y demostrados, se caracterizó el láser de cavidad externa mediante una integración híbrida. Para la caracterización del láser se usó un alineamiento activo entre el chip de ganancia QD y el chip de silicio con la cavidad externa, empleando el banco de alineamiento con montaje de acoplo horizontal. En una primera instancia se evaluó la integración híbrida con una cavidad externa sin filtro de anillo (figura 4.36(a)) para analizar el efecto de la cavidad FabryPerot y la respectiva separación de los modos de cavidad. La longitud de la guía de la cavidad externa era de $L_{W G E C}=160 \mu m$ y la reflectividad del DBR de $R=95 \%$. En la figura 4.36(b) se muestra el alineamiento entre el QD y la estructura de acoplo de la cavidad externa sin filtro. De esta manera, el punto de luz en la interfaz de salida se visualizó mediante la cámara de infrarrojo conectada al PC (figura 4.36(c)). El paso siguiente al alineamiento del QD en el puerto de entrada de la muestra fue remplazar el objetivo por una fibra óptica, que se alineó al puerto de salida. La fibra óptica se conectó primero a un medidor de potencia para optimizar el alineamiento y maximizar la potencia óptica a la salida. A continuación, la fibra se conectó a un analizador de espectros óptico con el cual se pudo visualizar el espectro de transmisión de la cavidad resultante con un pico máximo de potencia óptica correspondiente a un modo emitido por el dispositivo láser de alrededor de -15dBm (figura 4.36(d)). 
Capítulo 4: Fabricación y resultados experimentales del esquema propuesto de integración híbrida de láser con cavidad externa
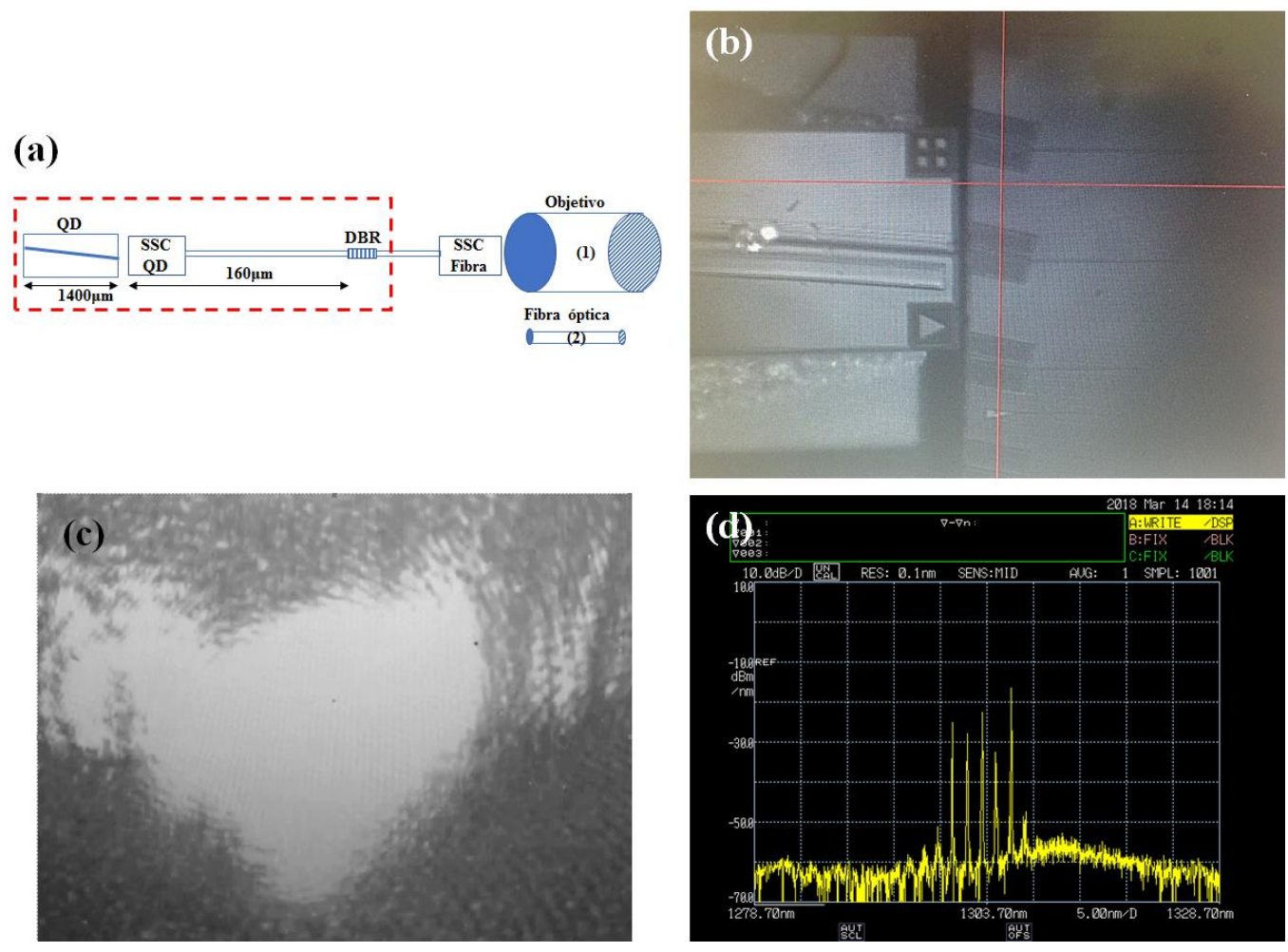

Figura 4.36: (a) Esquema de la cavidad Fabry-Perot formada por el QD y el DBR. (b) Alineamiento del QD y la estructura de acoplo en el chip de silicio. (c) Punto de luz captado y visualizado por la cámara de infrarrojo en la interfaz de salida de la muestra después del alineamiento activo del QD respecto a objetivo. (d) Espectro de salida del láser híbrido sin filtro en la cavidad.

El espectro de transmisión de la cavidad resultante tiene varios picos o modos de cavidad para los cuales se cumple la condición de amplitud y fase obteniéndose el efecto de amplificación de luz por emisión estimulada. Además, se analizó la separación de los modos de cavidad, observándose en el espectro de transmisión un patrón continuo de separaciones entre los modos excitados dentro de la cavidad. Sin embargo, se midieron dos patrones de separaciones de modos: uno mayor y persistente de $1.4 \mathrm{~nm}$ y otro menor de $0.2 \mathrm{~nm}$ (figura 4.37(a)). Al realizar el cálculo para obtener la separación de los modos de cavidad se observa que las medidas experimentales realizadas se aproximan y corresponden a modos de cavidad formados en la cavidad externa $\left(L_{W G E C}=160 \mu \mathrm{m}\right) \mathrm{de}$ alrededor de $1.3 \mathrm{~nm}$ y propiamente a los que se forman entre el QD y la cavidad externa $\left(L_{Q D}+L_{W G E C}=1560 \mu m\right.$ ) de alrededor de $0.16 \mathrm{~nm}$ (figura 4.37(b)), de manera que se produce un efecto no deseado de multi-cavidad por reflexiones indeseadas en la interfaz entre el chip de silicio y el QD. 


\subsection{Desarrollo y caracterización del láser con cavidad externa}

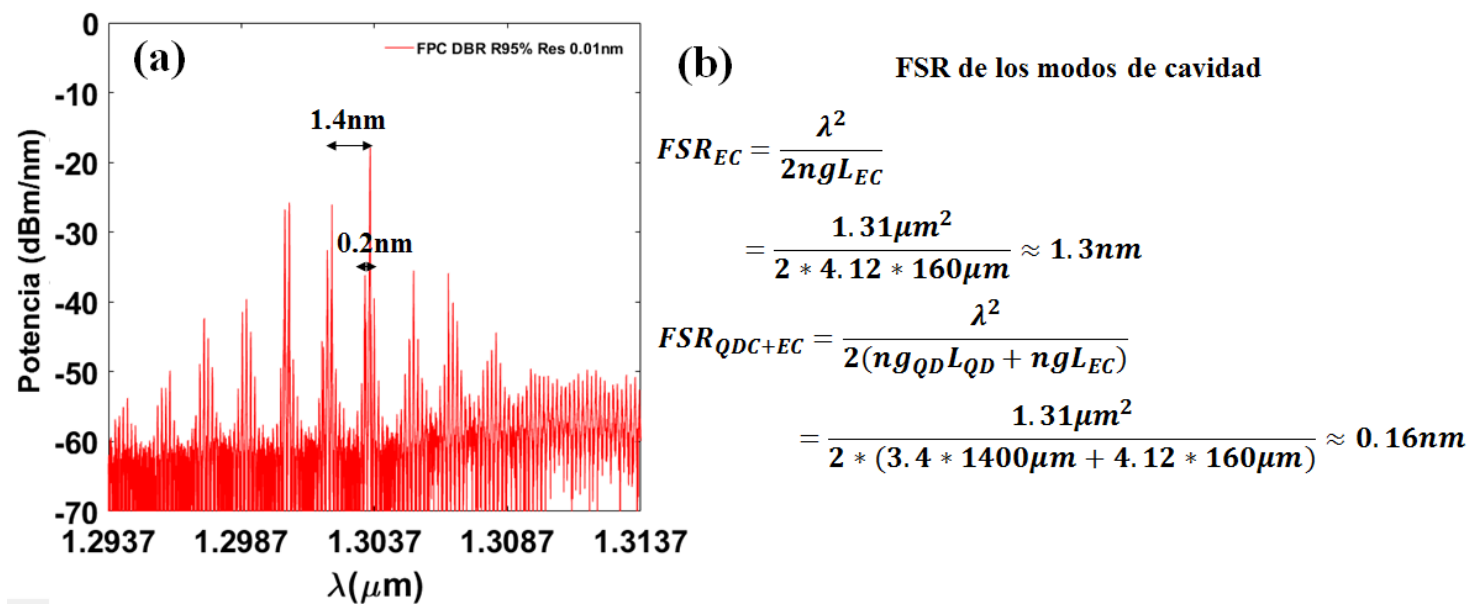

Figura 4.37: (a) Medición del espaciado de los modos excitados en la cavidad FP en el espectro de transmisión obtenido experimentalmente. (b) Cálculo de la separación entre modos.

Finalmente, la cavidad externa formada con todos los bloques de diseño incluyendo el filtro de anillo resonante y el DBR se fabricó y caracterizó. El GDS de la cavidad externa con todos los bloques diseñados se muestra en la figura 4.38(a). La longitud de la cavidad externa es de alrededor de $L_{W G E C}=371 \mu \mathrm{m}$ con lo que la separación de los modos de cavidad, formada entre el QD y la cavidad externa, es de alrededor de $0.14 \mathrm{~nm}$. Al igual que el caso anterior se realizó de manera inicial un alineamiento activo entre el QD y el chip de silicio con el objetivo (figura 4.38(b)). En la figura 4.38(c, d) se muestra el punto de luz captado por la cámara de infrarrojo cuando el QD se encuentra perfectamente desalineado y alineado, respectivamente, observándose un gran destello cuando el QD está totalmente alineado y se alcanza el régimen de emisión láser. La máxima potencia óptica medida respecto a objetivo es de $-6 \mathrm{dBm}$ con una tensión aplicada sobre el QD de $2.4 \mathrm{~V}$ y una corriente de $115 \mathrm{~mA}$. 
Capítulo 4: Fabricación y resultados experimentales del esquema propuesto de integración híbrida de láser con cavidad externa
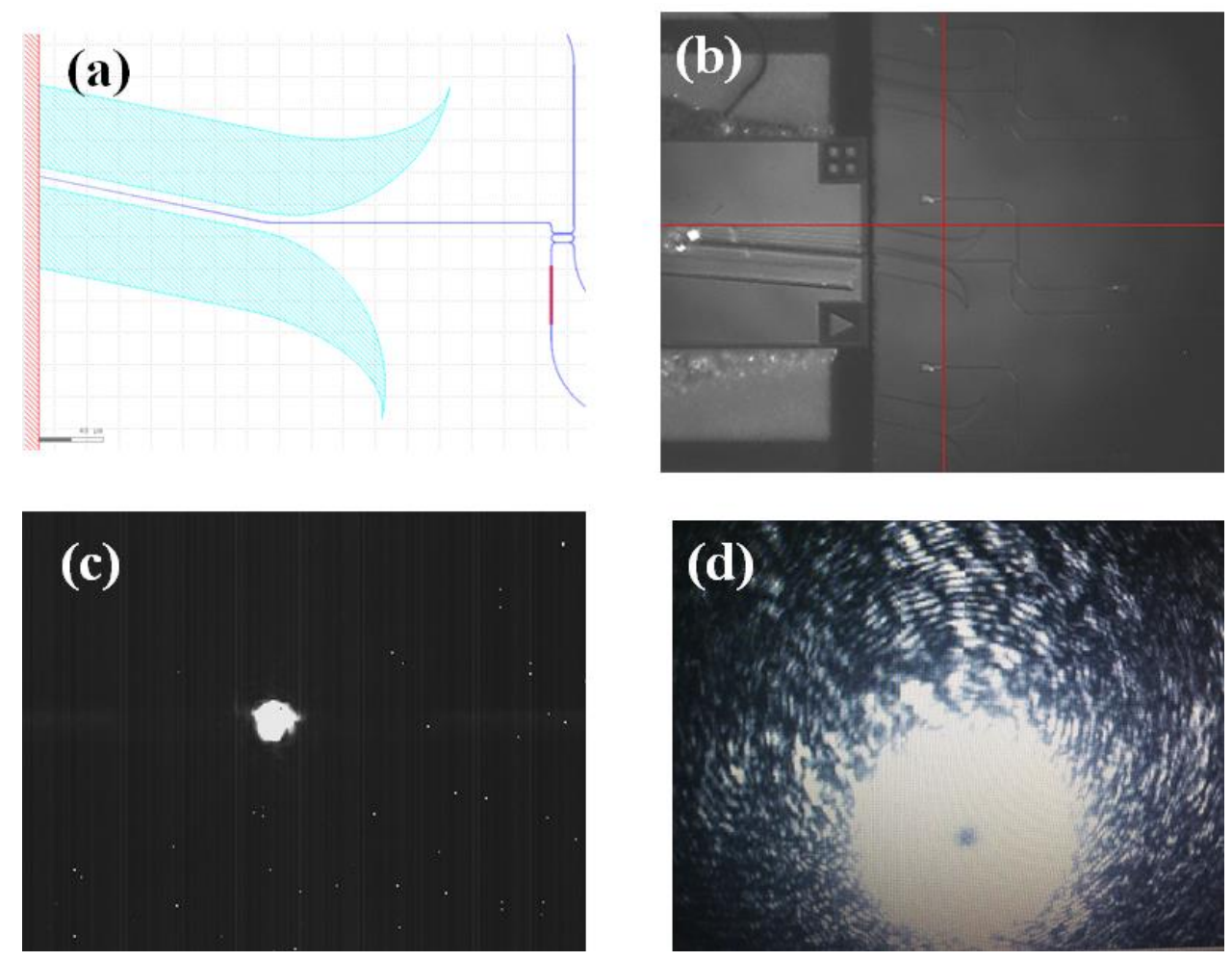

Figura 4.38: (a) GDS de la cavidad externa con todos los bloques de diseño incluidos. (b) Alineamiento activo del QD y el chip de silicio. (c) Punto de luz captado por la cámara de infrarrojo cuando el QD y la cavidad externa no están alineados, y (d) punto de luz y destello cuando están totalmente alineados y el régimen de emisión estimulada es alcanzado.

Finalmente, se alineó una fibra óptica en el puerto de salida, la cual se conectó al analizador de espectros para visualizar la repuesta del láser. Se obtuvo un solo modo excitado a una longitud de onda de alrededor de $1301 \mathrm{~nm}$ alcanzado la condición monomodo y con una relación de supresión de modo lateral o SMSR mayor a 30dB (figura 4.39(a)). En la figura 4.39(b) se muestra la curva de potencia óptica de salida del láser híbrido en función de la tensión aplicada y corriente suministrada al QD. Se observa que la curva tiene la forma característica de un láser consistente en una ganancia lineal a partir de un umbral de corriente de alrededor de 60mA, en el cual se pasa del estado de emisión espontánea a emisión estimulada. De esta manera, la potencia de salida del láser híbrido aumenta debido al incremento de corriente hasta llegar a un máximo. Sin embargo, la respuesta es oscilatoria tal y como se observa en la figura 4.39(b). Esto se debe principalmente al incremento de temperatura en el chip de ganancia QD debido a la mayor corriente, lo cual deteriora su funcionamiento y da lugar a un efecto no deseado de salto de modos o "mode-hopping" [101]. 

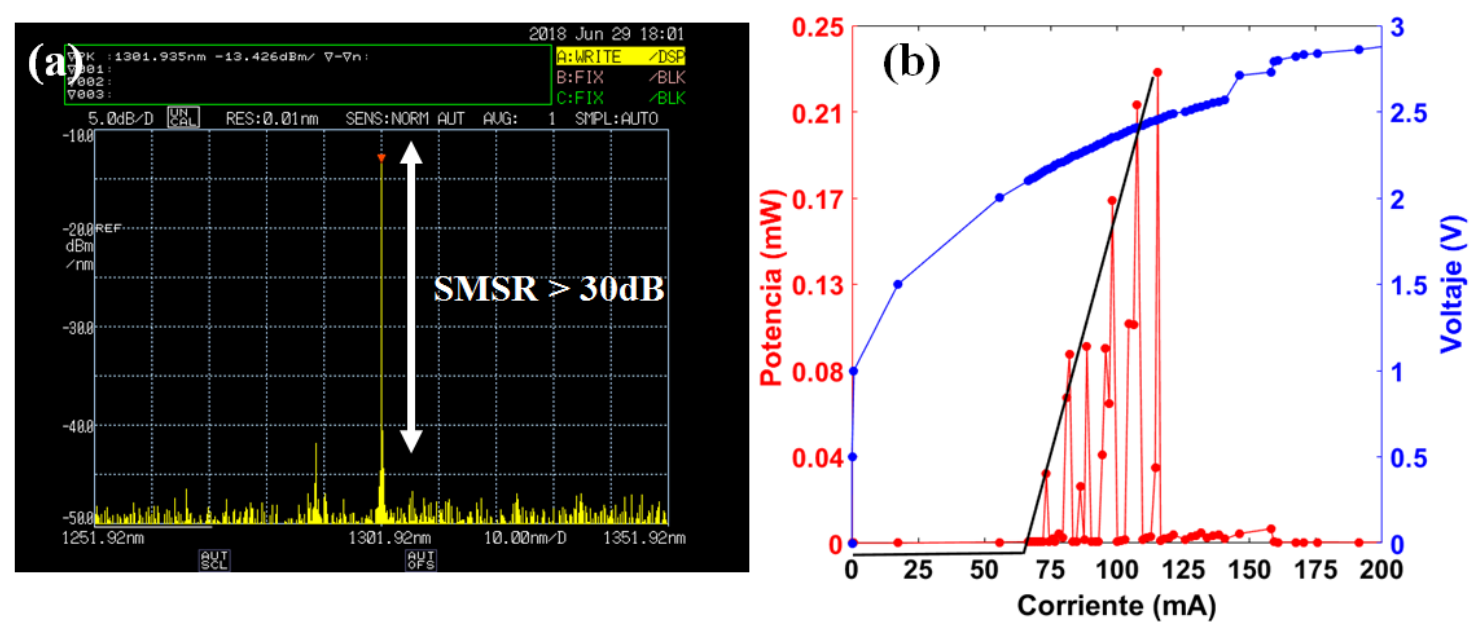

Figura 4.39: (a) Espectro medido del láser monomodo de cavidad externa. (b) Potencia de salida del láser de cavidad externa en función de la tensión y suministro de corriente aplicada al chip de ganancia QD.

De esta manera, se estimó la potencia del láser en la guía justo a la salida del DBR mediante la normalización respecto a las pérdidas de propagación en la guía que conecta al DBR con el puerto de salida $\alpha_{W G}=2.8 d B\left(L_{W G} \approx 5600 \mu m, \alpha=5 \frac{d B}{\mathrm{~cm}}\right)$ y las pérdidas de la estructura de acoplo a fibra $\alpha_{S S C}=4 d B$. El resultado obtenido fue una potencia de salida de $P_{\text {out }}=0.8 \mathrm{dBm}(1.2 \mathrm{~mW})$, tal como se observa en la figura 4.40. En definitiva, se consiguió demostrar satisfactoriamente el funcionamiento del láser de cavidad externa en la banda de $1310 \mathrm{~nm}$ y formado por la integración híbrida entre una fuente de luz de banda ancha y el chip de silicio.

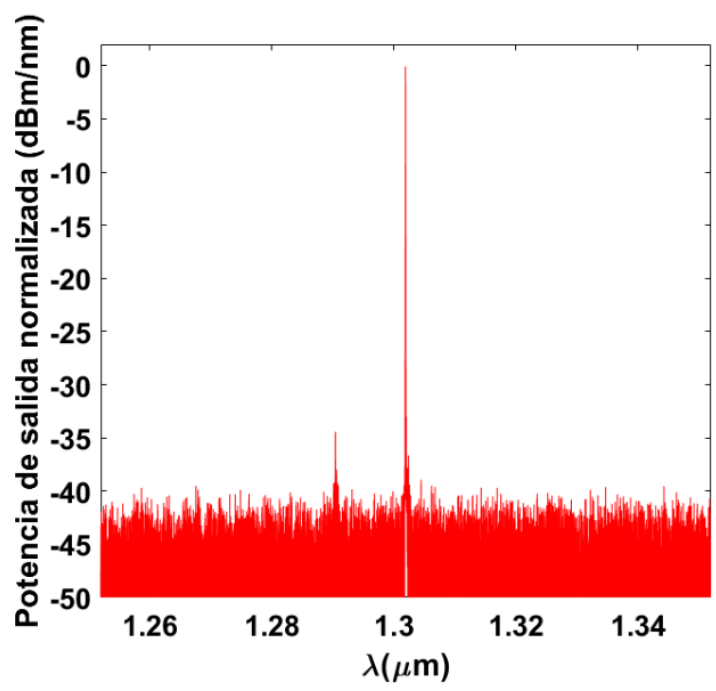

Figura 4.40: Espectro de potencia normalizado del láser híbrido. 



\section{Capítulo 5}

\section{Conclusiones y perspectivas futuras}

Esta tesis se enmarca en el área de Fotónica de Silicio y se ha centrado en el desarrollo e integración de fuentes de luz para aplicaciones de comunicaciones. Para ello, se diseñaron y estudiaron diversas estructuras, partiendo de estructuras de acoplamiento hasta el diseño de filtros ópticos y reflectores. El diseño, fabricación y caracterización de cada estructura se ha conseguido demostrar con éxito resaltando los siguientes resultados:

- Guías de onda ranurada tipo "slot" que permiten un alto confinamiento de la luz en una delgada capa de un material de menor índice de refracción que el silicio. Este tipo de estructuras se han propuesto para la implementación de láseres integrados monolíticamente. De esta forma, se diseñó una estructura de acoplo vertical sobre guía "slot" horizontal que opera sobre una longitud de onda de $1550 \mathrm{~nm}$ y en polarización TM reportando una alta eficiencia de acoplo de hasta el 52\% en guías asimétricas.

- Se estudió y diseñó estructuras de acoplo para acoplamiento a fibra óptica tanta en una configuración de acoplo vertical como horizontal y en la banda de comunicaciones $\mathrm{O}$. Se diseñó una red de difracción o grating y se caracterizó con eficiencias de acoplamiento de alrededor del $40 \%$ y un ancho de banda de operación a $3 \mathrm{~dB}$ de $54 \mathrm{~nm}$. De la misma manera, se diseñaron y caracterizaron convertidores de tamaño de modo alcanzando una eficiencia de acoplo de alrededor del $50 \%$ con un ancho de banda plano que cubre toda la banda $\mathrm{O}$.

- Se diseñó y caracterizó un filtro basado en anillo resonante con funcionamiento en la banda $\mathrm{O}$, de manera que se alcanzaron pérdidas de inserción menores a $1 \mathrm{~dB}$ y una relación de extinción de la señal mayor a $20 \mathrm{~dB}$ con un FSR de $10 \mathrm{~nm}$ y $12 \mathrm{~nm}$. El factor de calidad alcanzado en cada filtro es de alrededor de 3000 y 4000 obteniendo un ancho de banda a $3 \mathrm{~dB}$ en la resonancia de $0.4 \mathrm{~nm}$ y $0.3 \mathrm{~nm}$ respectivamente. El factor de acoplo se incrementó, manteniendo la separación entre la guía y el anillo, mediante la disminución de la anchura de la guía, reduciendo así el factor de confinamiento del modo propagado. 
- Se diseñó y caracterizó un reflector por medio de una red de difracción y se analizó en detalle su funcionamiento. Por medio de la implementación de cavidades FabryPerot se caracterizaron las estructuras y se demostraron reflectividades del $49 \%$ y $73 \%$.

- Se diseñó una estructura de acoplo horizontal por medio de cambio de tamaño de modo para acoplo de una fuente de luz externa QD a una guía SOI partiendo de un modelado matemático del haz de propagación de la fuente de luz en campo cercano. La eficiencia alcanzada es de alrededor del $30 \%$ observándose una alta sensibilidad al alineamiento en especial en el eje vertical.

- Se llevó a cabo un estudio para la sintonización del filtro de anillo dentro de la cavidad empleando calentadores sobre la estructura. También se realizó el estudio del desplazamiento de los modos de cavidad por medio de un calentador sobre una sección de la guía de la cavidad externa, demostrando el alineamiento y desalineamiento de los modos de cavidad respecto a la resonancia del filtro de anillo. Las potencias consumidas de forma experimental fueron entre $20 \mathrm{~mW}$ y $60 \mathrm{~mW}$.

- Se integró un fotodetector como parte de la cavidad externa, utilizado como punto de monitorización en una de las salidas del filtro de anillo. El fotodetector se integró sobre un acoplador de red de difracción para acoplo vertical y su funcionamiento fue demostrado con éxito.

- Finalmente, se demostró la integración híbrida mediante un alineamiento activo entre la fuente de luz (medio de ganancia) y el chip de silicio con la cavidad externa formada con todos los bloques de diseño. En una primera parte se formó una cavidad FabryPerot corroborando el espaciado de los modos de cavidad y obteniendo un espectro de emisión multimodo. Después, se incluyó el filtro de anillo en la cavidad de tal manera que se consiguió demostrar un láser de cavidad externa monomodo con una potencia de salida de alrededor de $1.2 \mathrm{~mW}$.

Las perspectivas futuras del trabajo de investigación pueden ser descritas en varias fases. Por una parte, el desarrollo de una tarjeta de circuito impreso (PCB) e integración con el chip de silicio de manera que se pueda realizar conexiones entre los electrodos de los calentadores fabricados en el chip de silicio y puertos de entrada en la PCB por medio de técnicas de encapsulado como lo es el "wire-bonding". Con lo cual, se prescindirá del uso de puntas sobre el chip de silicio para la sintonización del láser, conectando de manera directa las fuentes regulables de tensión en los puertos de salida de la PCB. De esta manera, se podrá hacer un control activo tanto en el calentador sobre el filtro de anillo resonante como en el calentador sobre una parte de la guía de la cavidad externa, para sintonizar la resonancia del anillo y alinear los modos de cavidad con la misma, maximizando la salida del láser. Por otra parte, integrar la fuente de luz QD en el chip de silicio, por medio de la técnica de encapsulado "flip-chip bonding", de manera que por medio de un alineamiento pasivo con la cavidad externa el láser de integración híbrida sea formado. Finalmente, con la integración del QD en el chip de silicio y de este con una $\mathrm{PCB}$, desarrollar un prototipo de láser sintonizable que opere en la banda $\mathrm{O}$ para diversas aplicaciones en comunicaciones ópticas, tales como modulación óptica, interconexiones ópticas entre otras. 



\section{Lista de Publicaciones}

\section{PUBLICACIONES EN REVISTA}

- R. Larrea, A. Gutiérrez, J. Hurtado, J. Ramírez, B. Garrido and P. Sanchis, "Compact focusing grating couplers for silicon horizontal slot waveguides", Optics Letters, vol. 42, no. 3, p. 490, 2017.

- R. Larrea, A. Gutierrez and P. Sanchis, "Design method for high performance grating couplers in photonic integrated circuits", Optical and Quantum Electronics, vol. 50, no. 9, 2018.

- R. Larrea, A. Gutierrez, A. Griol, A. Brimont and P. Sanchis, "Fiber-to-Chip Spot-Size Converter for Coupling to Silicon Waveguides in the O-band", accepted in IEEE Photonics Technology Letters, pp. 1-4, 2018.

\section{PUBLICACIONES EN CONGRESO}

- R. Larrea, A. Gutierrez and P. Sanchis, "Optimized grating couplers on SOI for high performance operation at $1310 \mathrm{~nm}$ wavelength", 2016 IEEE 13th International Conference on Group IV Photonics (GFP), 2016.

- R. Larrea-Luzuriaga, A. Gutierrez and P. Sanchis, "Analytical strategy to achieve optimized grating couplers with high precision for both TE and TM polarizations on SOI platform", 2016 IEEE Ecuador Technical Chapters Meeting (ETCM), 2016.

- R. Larrea-Luzuriaga, A. Gutierrez and P. Sanchis, "On the design of silicon microring filters with wide free spectral range for the O-band", 2017 IEEE Second Ecuador Technical Chapters Meeting (ETCM), 2017.

\section{OTRAS PUBLICACIONES}

- R. Larrea-Luzuriaga, J. Jiménez, S. Sendra and J. Lloret, " Comparative Study of Routing Protocols in Ring Topologies using GNS3 ", ICIMP 2016: The Eleventh International Conference on Internet Monitoring and Protection, 2016.

- I. Olivares, L. Sánchez, J. Parra, R. Larrea, A. Griol, M. Menghini, P. Homm, L. Jang, B. van Bilzen, J. Seo, J. Locquet and P. Sanchis, "Optical switching in 
hybrid $\mathrm{VO}_{2} / \mathrm{Si}$ waveguides thermally triggered by lateral microheaters", Optics Express, vol. 26, no. 10, p. 12387, 2018. 



\section{Bibliografía}

[1] M. Ohtsu, Principles of nanophotonics. Boca Raton: CRC Press/Taylor \& Francis, 2008, pp. 1-16.

[2] G. Moore, "Cramming More Components Onto Integrated Circuits", Proceedings of the IEEE, vol. 86, no. 1, pp. 82-85, 1998.

[3] M. Naruse, Nanophotonic information physics: nanointelligence and nanophotonic computing. Springer, 2008, pp. 1-7.

[4] D. Dai, J. Bauters and J. Bowers, "Passive technologies for future large-scale photonic integrated circuits on silicon: polarization handling, light non-reciprocity and loss reduction", Light: Science \& Applications, vol. 1, no. 3, pp. e1-e1, 2012.

[5] D. Thomson, F. Gardes, J. Fedeli, S. Zlatanovic, Y. Hu, B. Kuo, E. Myslivets, N. Alic, S. Radic, G. Mashanovich and G. Reed, "50-Gb/s Silicon Optical Modulator", IEEE Photonics Technology Letters, vol. 24, no. 4, pp. 234-236, 2012.

[6] L. Vivien, A. Polzer, D. Marris-Morini, J. Osmond, J. Hartmann, P. Crozat, E. Cassan, C. Kopp, H. Zimmermann and J. Fédéli, "Zero-bias 40Gbit/s germanium waveguide photodetector on silicon", Optics Express, vol. 20, no. 2, p. 1096, 2012.

[7] P. Liao, "Ultradense Silicon Photonic Interface for Optical Interconnection," Ieee Photonics Technology Letters, Vol. 27, No. 7, April 1, 2015.

[8] E. Palik and G. Ghosh, Handbook of optical constants of solids, 3rd ed. San Diego: Academic Press, 1998, pp. 547-570.

[9] G. Ghosh, Handbook of thermo-optic coefficients of optical materials with applications. San Diego, Calif. [u.a.]: Acad. Press, 1998, pp. 116-231.

[10] H. Zimmermann, Integrated silicon optoelectronics. Heidelberg: Springer, 2010, pp. $1-4$. 
[11] W. Bogaerts, P. Dumon, M. Fiers, A. Ribeiro and M. Vanslembrouck, "Silicon Photonics Integrated Design", International conference on fiber optics and photonics, pp. 1-3, 2012.

[12] S. Fathpour and B. Jalali, Silicon photonics for telecommunications and biomedicine. Boca Raton, FL: CRC Press, 2012, pp. 1-49.

[13] K. Yamada, "Silicon Photonic Wire Waveguides: Fundamentals and Applications", Topics in Applied Physics, pp. 1-29, 2010.

[14] L. Vivien, Handbook of silicon photonics. Boca Raton: Taylor \& Francis, 2013, pp. 97-138.

[15] L. Jia, H. Zhou, T. Liow, J. Song, Y. Huang, X. Tu, X. Luo, C. Li, Q. Fang, M. Yu and G. Lo, "Analysis of the polarization rotation effect in the inversely tapered spot size converter", Optics Express, vol. 23, no. 21, p. 27776, 2015.

[16] M. Galarza, "Leaky-Waveguide-Based Integrated Spot-Size Converters for Alignment-Tolerant Fiber-Coupling", Ph.D, Universiteit Gent, 2018.

[17] J. Cardenas, C. Poitras, K. Luke, L. Luo, P. Morton and M. Lipson, "High Coupling Efficiency Etched Facet Tapers in Silicon Waveguides", IEEE Photonics Technology Letters, vol. 26, no. 23, pp. 2380-2382, 2014.

[18] Y. Maegami, R. Takei, E. Omoda, T. Amano, M. Okano, M. Mori, T. Kamei and Y. Sakakibara, "Spot-size converter with a SiO_2 spacer layer between tapered Si and SiON waveguides for fiber-to-chip coupling", Optics Express, vol. 23, no. 16, p. 21287, 2015.

[19] K. Shiraishi and C. Tsai, "A Spot-Size Converter With Concatenated Up- and Down-Tapers Followed by a Thin Slab-Waveguide", IEEE Photonics Technology Letters, vol. 28, no. 4, pp. 485-488, 2016.

[20] M. Galarza, K. De Mesel, S. Verstuyft, C. Aramburu, M. Lopez-Amo, I. Moerman, P. Van Daele and R. Baets, "A new spot-size converter concept using fiber-matched antiresonant reflecting optical waveguides", Journal of Lightwave Technology, vol. 21, no. 1, pp. 269-274, 2003.

[21] N. Hatori, T. Shimizu, M. Okano, M. Ishizaka, T. Yamamoto, Y. Urino, M. Mori, T. Nakamura and Y. Arakawa, "A Hybrid Integrated Light Source on a Silicon Platform Using a Trident Spot-Size Converter", Journal of Lightwave Technology, vol. 32, no. 7, pp. 1329-1336, 2014.

[22] C. Elachi, "Waves in active and passive periodic structures: A review", Proceedings of the IEEE, vol. 64, no. 12, pp. 1666-1698, 1976. 


\section{Bibliografía}

[23] N. Bonod and J. Neauport, "Diffraction gratings: from principles to applications in high-intensity lasers", Advances in Optics and Photonics, vol. 8, no. 1, p. 156, 2016.

[24] R. Halir, P. Bock, P. Cheben, A. Ortega-Moñux, C. Alonso-Ramos, J. Schmid, J. Lapointe, D. Xu, J. Wangüemert-Pérez, Í. Molina-Fernández and S. Janz, "Waveguide sub-wavelength structures: a review of principles and applications", Laser \& Photonics Reviews, vol. 9, no. 1, pp. 25-49, 2014.

[25] C. Alonso-Ramos, A. Ortega-Moñux, I. Molina-Fernández, P. Cheben, L. ZavargoPeche, R. Halir, S. Janz, D. Xu, N. Kim and B. Lamontagne, "Grating couplers for thick SOI rib waveguides", Optical and Quantum Electronics, vol. 44, no. 12-13, pp. 535-540, 2012.

[26] C. Alonso-Ramos, A. Ortega-Moñux, R. Halir, L. Zavargo-Peche, I. MolinaFernández, P. Cheben, D. Xu and S. Janz, "Grating couplers in thick rib SOI waveguides for TE and TM polarizations", Silicon Photonics and Photonic Integrated Circuits III, 2012.

[27] Vivien, Pascal, Lardenois, Marris-Morini, Cassan, Grillot, Laval, Fedeli and Loubna El Melhaoui, "Light injection in SOI microwaveguides using highefficiency grating couplers", Journal of Lightwave Technology, vol. 24, no. 10, pp. 3810-3815, 2006.

[28] L. He, Y. He, A. Pomerene, C. Hill, S. Ocheltree, T. Baehr-Jones and M. Hochberg, "Ultrathin Silicon-on-Insulator Grating Couplers", IEEE Photonics Technology Letters, vol. 24, no. 24, pp. 2247-2249, 2012.

[29] M. Cabezón, I. Garcés, A. Villafranca, J. Pozo, P. Kumar and A. Kaźmierczak, "Silicon-on-insulator chip-to-chip coupling via out-of-plane or vertical grating couplers", Applied Optics, vol. 51, no. 34, p. 8090, 2012.

[30] R. Halir, P. Cheben, S. Janz, D. Xu, Í. Molina-Fernández and J. Wangüemert-Pérez, "Waveguide grating coupler with subwavelength microstructures", Optics Letters, vol. 34, no. 9, p. 1408, 2009.

[31] N. Ayotte, K. Bédard and S. LaRochelle, "Single-etch step grating couplers for SOI waveguides", Photonics North 2013, 2013.

[32] M. Antelius, K. Gylfason and H. Sohlström, "An apodized SOI waveguide-to-fiber surface grating coupler for single lithography silicon photonics", Optics Express, vol. 19, no. 4, p. 3592, 2011. 
[33] G. Dabos, D. Kalavrouziotis, J. Bolten, A. Prinzen, N. Pleros and D. Tsiokos, "Costeffective single-etched TM-mode SOI grating couplers for broadband perfectly vertical coupling", Silicon Photonics IX, 2014.

[34] J. Klamkin and B. Song, "3-D Hybrid Laser Integration for Silicon Photonics | Optics \& Photonics News", Osa-opn.org, 2018. [Online]. Available: https://www.osa-opn.org/home/articles/volume_27/december_2016/extras/3d_hybrid_laser_integration_for_silicon_photonics/.

[35] L. Agazzi, J. Bradley, M. Dijkstra, F. Ay, G. Roelkens, R. Baets, K. Wörhoff and M. Pollnau, "Monolithic integration of erbium-doped amplifiers with silicon-oninsulator waveguides", Optics Express, vol. 18, no. 26, p. 27703, 2010.

[36] H. Jayatilleka, A. Nasrollahy-Shiraz and A. Kenyon, "Electrically pumped silicon waveguide light sources", Optics Express, vol. 19, no. 24, p. 24569, 2011.

[37] Ramírez, Y. Berencén, F. Lupi, D. Navarro-Urrios, A. Anopchenko, A. Tengattini, N. Prtljaga, L. Pavesi, P. Rivallin, J. Fedeli and B. Garrido, "Electrical pump \& probe and injected carrier losses quantification in Er doped Si slot waveguides", Optics Express, vol. 20, no. 27, p. 28808, 2012.

[38] Ramírez, F. Ferrarese Lupi, Y. Berencén, A. Anopchenko, J. Colonna, O. Jambois, J. Fedeli, L. Pavesi, N. Prtljaga, P. Rivallin, A. Tengattini, D. Navarro-Urrios and B. Garrido, "Er-doped light emitting slot waveguides monolithically integrated in a silicon photonic chip", Nanotechnology, vol. 24, no. 11, p. 115202, 2013.

[39] A. Tengattini, D. Gandolfi, N. Prtljaga, A. Anopchenko, J. Ramirez, F. Lupi, Y. Berencen, D. Navarro-Urrios, P. Rivallin, K. Surana, B. Garrido, J. Fedeli and L. Pavesi, "Toward a $1.54 \mu \mathrm{m}$ Electrically Driven Erbium-Doped Silicon Slot Waveguide and Optical Amplifier", Journal of Lightwave Technology, vol. 31, no. 3, pp. 391-397, 2013.

[40] Bradley, E. Hosseini, Purnawirman, Z. Su, T. Adam, G. Leake, D. Coolbaugh and M. Watts, "Monolithic erbium- and ytterbium-doped microring lasers on silicon chips", Optics Express, vol. 22, no. 10, p. 12226, 2014.

[41] Z. Zhou, B. Yin and J. Michel, "On-chip light sources for silicon photonics", Light: Science \& Applications, vol. 4, no. 11, pp. e358-e358, 2015.

[42] Liu, X. Sun, D. Pan, X. Wang, L. Kimerling, T. Koch and J. Michel, "Tensilestrained, n-type $\mathrm{Ge}$ as a gain medium for monolithic laser integration on $\mathrm{Si}^{\prime}$, Optics Express, vol. 15, no. 18, p. 11272, 2007. 


\section{Bibliografía}

[43] Liu, X. Sun, R. Camacho-Aguilera, L. Kimerling and J. Michel, "Ge-on-Si laser operating at room temperature", Optics Letters, vol. 35, no. 5, p. 679, 2010.

[44] R. Camacho-Aguilera, Y. Cai, N. Patel, J. Bessette, M. Romagnoli, L. Kimerling and J. Michel, "An electrically pumped germanium laser", Optics Express, vol. 20, no. 10, p. 11316, 2012.

[45] C. Cornet, Y. Léger and C. Robert, Integrated lasers on silicon. London Kidlington: Oxford: ISTE Press, Ltd. Elsevier, 2016, pp. 73-104.

[46] H. Park, A. Fang, S. Kodama and J. Bowers, "Hybrid silicon evanescent laser fabricated with a silicon waveguide and III-V offset quantum wells", Optics Express, vol. 13, no. 23, p. 9460, 2005.

[47] A. Fang, H. Park, O. Cohen, R. Jones, M. Paniccia and J. Bowers, "Electrically pumped hybrid AlGaInAs-silicon evanescent laser", Optics Express, vol. 14, no. 20, p. 9203, 2006.

[48] X. Sun, A. Zadok, M. Shearn, K. Diest, A. Ghaffari, H. Atwater, A. Scherer and A. Yariv, "Electrically pumped hybrid evanescent Si/InGaAsP lasers", Optics Letters, vol. 34, no. 9, p. 1345, 2009.

[49] K. Tanabe, K. Watanabe and Y. Arakawa, "III-V/Si hybrid photonic devices by direct fusion bonding", Scientific Reports, vol. 2, no. 1, 2012.

[50] A. Liu, C. Zhang, J. Norman, A. Snyder, D. Lubyshev, J. Fastenau, A. Liu, A. Gossard and J. Bowers, "High performance continuous wave $1.3 \mu \mathrm{m}$ quantum dot lasers on silicon", Applied Physics Letters, vol. 104, no. 4, p. 041104, 2014.

[51] S. Yang, "Hybrid laser integration for silicon photonics platform", PhD. Thesis, University of Delaware, 2015.

[52] D. Paschotta, "Encyclopedia of Laser Physics and Technology - quantum wells", Rp-photonics.com, 2018. [Online]. Available: https://www.rpphotonics.com/quantum_wells.html?s=ak.

[53] D. Paschotta, "Encyclopedia of Laser Physics and Technology - quantum dots, semiconductor nanocrystal, Stranski-Krastanov growth, colloidally suspended quantum dots", Rp-photonics.com, 2018. [Online]. Available: https://www.rpphotonics.com/quantum_dots.html.

[54] Z. Zhou, B. Yin and J. Michel, "On-chip light sources for silicon photonics", Light: Science \& Applications, vol. 4, no. 11, pp. e358-e358, 2015.

[55] A. Fang, E. Lively, Y. Kuo, D. Liang and J. Bowers, "A distributed feedback silicon evanescent laser", Optics Express, vol. 16, no. 7, p. 4413, 2008. 
[56] A. Fang, B. Koch, R. Jones, E. Lively, Di Liang, Ying-Hao Kuo and J. Bowers, "A Distributed Bragg Reflector Silicon Evanescent Laser", IEEE Photonics Technology Letters, vol. 20, no. 20, pp. 1667-1669, 2008.

[57] A. Zilkie, P. Seddighian, B. Bijlani, W. Qian, D. Lee, S. Fathololoumi, J. Fong, R. Shafiiha, D. Feng, B. Luff, X. Zheng, J. Cunningham, A. Krishnamoorthy and M. Asghari, "Power-efficient III-V/Silicon external cavity DBR lasers", Optics Express, vol. 20, no. 21, p. 23456, 2012.

[58] S. Tanaka, S. Jeong, S. Sekiguchi, T. Kurahashi, Y. Tanaka and K. Morito, "Highoutput-power, single-wavelength silicon hybrid laser using precise flip-chip bonding technology", Optics Express, vol. 20, no. 27, p. 28057, 2012.

[59] X. Zheng, S. Lin, Y. Luo, J. Yao, G. Li, S. Djordjevic, J. Lee, H. Thacker, I. Shubin, K. Raj, J. Cunningham and A. Krishnamoorthy, "Efficient WDM Laser Sources Towards Terabyte/s Silicon Photonic Interconnects", Journal of Lightwave Technology, vol. 31, no. 24, pp. 4142-4154, 2013.

[60] S. Keyvaninia, G. Roelkens, D. Van Thourhout, C. Jany, M. Lamponi, A. Le Liepvre, F. Lelarge, D. Make, G. Duan, D. Bordel and J. Fedeli, "Demonstration of a heterogeneously integrated III-V/SOI single wavelength tunable laser", Optics Express, vol. 21, no. 3, p. 3784, 2013.

[61] S. Tanaka, T. Akiyama, S. Sekiguchi and K. Morito, "Silicon Photonics Optical Transmitter Technology for Tb/s-class I/O Co-packaged with CPU", Fujitsu.com, 2018.

[Online].

Available:

http://www.fujitsu.com/global/documents/about/resources/publications/fstj/archiv es/vol50-1/paper19.pdf. [Accessed: 23- Mar- 2018].

[62] N. Hatori, T. Shimizu, M. Okano, M. Ishizaka, T. Yamamoto, Y. Urino, M. Mori, T. Nakamura and Y. Arakawa, "A Hybrid Integrated Light Source on a Silicon Platform Using a Trident Spot-Size Converter", Journal of Lightwave Technology, vol. 32, no. 7, pp. 1329-1336, 2014.

[63] T. Shimizu, N. Hatori, M. Kurihara, Y. Urino, T. Yamamoto, T. Nakamura and Y. Arakawa, "Optical Characteristics of a Multichannel Hybrid Integrated Light Source for Ultra-High-Bandwidth Optical Interconnections", Photonics, vol. 2, no. 4, pp. 1131-1138, 2015.

[64] J. Lee, I. Shubin, J. Yao, J. Bickford, Y. Luo, S. Lin, S. Djordjevic, H. Thacker, J. Cunningham, K. Raj, X. Zheng and A. Krishnamoorthy, "High power and widely 


\section{Bibliografía}

tunable Si hybrid external-cavity laser for power efficient Si photonics WDM links", Optics Express, vol. 22, no. 7, p. 7678, 2014.

[65] H. Guan, A. Novack, T. Galfsky, Y. Ma, S. Fathololoumi, A. Horth, T. Huynh, J. Roman, R. Shi, M. Caverley, Y. Liu, T. Baehr-Jones, K. Bergman and M. Hochberg, "Widely-tunable, narrow-linewidth III-V/silicon hybrid external-cavity laser for coherent communication", Optics Express, vol. 26, no. 7, p. 7920, 2018.

[66] G. de Valicourt, C. Chang, M. Eggleston, A. Melikyan, C. Zhu, J. Lee, J. Simsarian, S. Chandrasekhar, J. Sinsky, K. Kim, P. Dong, A. Maho, A. Verdier, R. Brenot and Y. Chen, "Photonic Integrated Circuit Based on Hybrid III-V/Silicon Integration", Journal of Lightwave Technology, vol. 36, no. 2, pp. 265-273, 2018.

[67] S. Zhu and G.-Qiang Lo, "Vertically Stacked Multilayer Photonics on Bulk Silicon Toward Three-Dimensional Integration,” J. Light. Tech. 34, 386, 2016.

[68] V. R. Almeida, Q. Xu, C. A. Barrios, and M. Lipson, "Guiding and confining light in void nanostructure," Opt. Lett. 29, 1209, 2004.

[69] R. Sun, P. Dong, N. Feng, C. Hong, J. Michel, M. Lipson, and L. Kimerling, "Horizontal single and multiple slot waveguides: optical transmission at $\lambda=1550$ nm,” Opt. Express 15, 17967, 2007.

[70] A. Martinez, J. Blasco, P. Sanchis, J. V. Galan, J. Garca-Ruperez, E. Jordana, P. Gautier, Y. Lebour, S. Hernandez, R. Spano, R. Guider, N. Daldosso, B. Garrido, J. M. Fedeli, L. Pavesi, J. Marti, "Ultrafast All-Optical Switching in a SiliconNanocrystal-Based Silicon Slot Waveguide at Telecom Wavelengths", Nano Letters, 10, 1506, 2010.

[71] A. Tengattini, D. Gandolfi, N. Prtljaga, A. Anopchenko, J. M. Ramírez, F. Ferrarese Lupi, Y. Berencén, D. Navarro-Urrios, P. Rivallin, K. Surana, B. Garrido, J.-M. Fedeli, and L. Pavesi, "Toward a $1.54 \mu \mathrm{m}$ Electrically Driven Erbium-Doped Silicon Slot Waveguide and Optical Amplifier," J. Light. Tech. 31, 391, 2013.

[72] J. M. Ramírez, F. Ferrarese Lupi, Y. Berencén, A. Anopchenko, J. P. Colonna, O. Jambois., J. M. Fedeli, L. Pavesi, N. Prtljaga, P. Rivallin, A. Tengattini, D. NavarroUrrios and B. Garrido, "Er-doped light emitting slot waveguides monolithically integrated in a silicon photonic chip," Nanotech. 24, 115202, 2013.

[73] R. Halir, P. Cheben, S. Janz, D.-Xia Xu, I. Molina-Fernández, and J. G. Wangüemert-Pérez, "Waveguide grating coupler with subwavelength microstructures," Opt. Lett. 34, 1408, 2009. 
[74] B. Schmid, A. Petrov, and M. Eich, "Optimized grating coupler with fully etched slots," Opt. Express 17, 11066, 2009.

[75] Z. Xiao, F. Luan, T.-Y. Liow, J. Zhang, and P. Shum, "Design for broadband highefficiency grating couplers," Opt. Lett. 37, 530, 2012.

[76] D. Benedikovic, P. Cheben, J. H. Schmid, D.-X. Xu, J. Lapointe, S. Wang, R. Halir, A. Ortega-Moñux, S. Janz, and M. Dado, "High-efficiency single etch step apodized surface grating coupler using subwavelength structure," Laser Photonics Rev. 8, L93, 2014.

[77] R. Halir, P. Bock, P. Cheben, A. Ortega-Moñux, C. Alonso-Ramos, J. H. Schmid, J. Lapointe, D.-X. Xu, J. G. Wangüemert-Pérez, I. Molina-Fernández, and S. Janz, "Waveguide sub-wavelength structures: a review of principles and applications," Laser Photonics Rev. 9, 25, 2015.

[78] J. V. Galan, P. Sanchis, J. Blasco, J. Marti, "Study of High Efficiency Grating Couplers for Silicon-Based Horizontal Slot Waveguides," Phot. Tech. Lett. 20, 985, 2008 .

[79] J. V. Galan, P. Sanchis, J. Blasco, A. Martinez, J. Marti, J. M. Fedeli, E. Jordana, P. Gautier, M. Perrin, "Silicon sandwiched slot waveguide grating couplers," Electronics Lett. 45, 262, 2009.

[80] J. Covey, R.T. Chen, "Efficient perfectly vertical fiber-to-chip grating coupler for silicon horizontal multiple slot waveguides,” Opt. Express 21, 10886 (2013).

[81] J. Harris, R. Winn and D. Dalgoutte, "Theory and Design of Periodic Couplers", Applied Optics, vol. 11, no. 10, p. 2234, 1972.

[82] L. Vivien, D. Pascal, S. Lardenois, D. Marris-Morini, E. Cassan, F. Grillot, S. Laval, J. Fedeli and Loubna El Melhaoui, "Light injection in SOI microwaveguides using high-efficiency grating couplers", Journal of Lightwave Technology, vol. 24, no. 10, pp. 3810-3815, 2006.

[83] F. Van Laere, T. Claes, J. Schrauwen, S. Scheerlinck, W. Bogaerts, D. Taillaert, L. O'Faolain, D. Van Thourhout and R. Baets, "Compact Focusing Grating Couplers for Silicon-on-Insulator Integrated Circuits", IEEE Photonics Technology Letters, vol. 19, no. 23, pp. 1919-1921, 2007.

[84] D. Paschotta, "Encyclopedia of Laser Physics and Technology - M2 factor, laser beam, quality factor, beam divergence, caustic, ISO Standard 11146", Rpphotonics.com, 2018. [Online]. Available: https://www.rpphotonics.com/m2_factor.html. [Accessed: 06- Apr- 2018]. 


\section{Bibliografía}

[85] "Technical-Library", Cvilaseroptics.com, 2018. [Online]. Available: https://www.cvilaseroptics.com/file/general/All_About_Gaussian_Beam_Optics WEB.pdf. [Accessed: 06- Apr- 2018].

[86] H. Sun, Laser diode beam basics, manipulations and characterizations. Dordrecht: Springer, 2012, pp. 21-37.

[87] RsoftCAD 12.0 User Guide, Rsoft Design Group, Inc. 2013, pp. 213-216.

[88] Ye, C. (2004). Tunable external cavity diode lasers. Singapore: World Scientific Publishing, pp.107-123.

[89] Coldren, L., Corzine, S. and Mashanovitch, M. (2012). Diode Lasers and Photonic Integrated Circuits, 2nd Edition. John Wiley \& Sons, pp.91-110.

[90] Bogaerts, W. (2004). Nanophotonic Waveguides and Photonic Crystals in Siliconon-Insulator. Ph.D. Universiteit Gent, pp. 193-197.

[91] W. Bogaerts, P. De Heyn, T. Van Vaerenbergh, K. De Vos, S. Kumar Selvaraja, T. Claes, P. Dumon, P. Bienstman, D. Van Thourhout and R. Baets, "Silicon microring resonators", Laser \& Photonics Reviews, vol. 6, no. 1, pp. 47-73, 2011.

[92] H. Venghaus, Wavelength filters in fibre optics. Berlin: Springer, 2006, pp. 341361.

[93] P. Yupapin, P. Saeung and C. Li, "Characteristics of complementary ring-resonator add/drop filters modeling by using graphical approach", Optics Communications, vol. 272, no. 1, pp. 81-86, 2007.

[94] D. Rabus, Integrated ring resonators. Berlin: Springer, 2007, pp. 3-18.

[95] Hohlfeld, D. (2005). Silicon-based Tunable Optical Filters. Ph.D. Universität Freiburg im Breisgau.

[96] Hong, G. (2005). Reflectance Measurement and Modelling of High Reflectivity Distributed Bragg Reflector (DBR) Stacks. MSc. University of Hull.

[97] Kwong, H. (1996). Design and Fabrication of Distributed Bragg Reflectors for Vertical-Cavity Surface-Emitting Lasers. MSc. Masschusetts Institute of Technology.

[98] Docter, B. (2009). Deeply-etched DBR mirrors for photonic integrated circuits and tunable lasers. Technische Universiteit Eindhoven, pp.1-29.

[99] Chen, R. and Choi, C. (2007). Optical interconnects. [San Rafael, Calif.]: Morgan \& Claypool Publishers, pp.9-12. 
[100] J. Galan, P. Sanchis, J. Blasco and J. Marti, "Study of High Efficiency Grating Couplers for Silicon-Based Horizontal Slot Waveguides", IEEE Photonics Technology Letters, vol. 20, no. 12, pp. 985-987, 2008.

[101] Heumier, T. and Carlsten, J. (2018). Mode Hopping in Semiconductor Laser Diodes - AN08. [online] Newport Corporation. Available at: https://www.newport.com/medias/sys_master/images/images/h4b/h48/879704958 5694/AN08-Mode-Hopping-in-Semiconductor-Laser-Diodes.pdf 